\title{
NORM ONE TORI AND HASSE NORM PRINCIPLE
}

\author{
AKINARI HOSHI, KAZUKI KANAI, AND AIICHI YAMASAKI
}

\begin{abstract}
Let $k$ be a field and $T$ be an algebraic $k$-torus. In 1969, over a global field $k$, Voskresenskii proved that there exists an exact sequence $0 \rightarrow A(T) \rightarrow H^{1}(k, \operatorname{Pic} \bar{X})^{\vee} \rightarrow \amalg(T) \rightarrow 0$ where $A(T)$ is the kernel of the weak approximation of $T, \amalg(T)$ is the Shafarevich-Tate group of $T, X$ is a smooth $k$-compactification of $T$, $\bar{X}=X \times_{k} \bar{k}, \operatorname{Pic} \bar{X}$ is the Picard group of $\bar{X}$ and $\vee$ stands for the Pontryagin dual. On the other hand, in 1963, Ono proved that for the norm one torus $T=R_{K / k}^{(1)}\left(\mathbb{G}_{m}\right)$ of $K / k, \amalg(T)=0$ if and only if the Hasse norm principle holds for $K / k$. First, we determine $H^{1}(k, \operatorname{Pic} \bar{X})$ for algebraic $k$-tori $T$ up to dimension 5 . Second, we determine $H^{1}(k, \operatorname{Pic} \bar{X})$ for norm one tori $T=R_{K / k}^{(1)}\left(\mathbb{G}_{m}\right)$ with $[K: k]=n \leq 15$ and $n \neq 12$. We also show that $H^{1}(k, \operatorname{Pic} \bar{X})=0$ for $T=R_{K / k}^{(1)}\left(\mathbb{G}_{m}\right)$ when the Galois group of the Galois closure of $K / k$ is the Mathieu group $M_{n} \leq S_{n}$ with $n=11,12,22,23,24$. Third, we give a necessary and sufficient condition for the Hasse norm principle for $K / k$ with $[K: k]=n \leq 15$ and $n \neq 12$. As applications of the results, we get the group $T(k) / R$ of $R$-equivalence classes over a local field $k$ via Colliot-Thélène and Sansuc's formula and the Tamagawa number $\tau(T)$ over a number field $k$ via Ono's formula $\tau(T)=\left|H^{1}(k, \widehat{T})\right| /|\amalg(T)|$.
\end{abstract}

\section{CONTENTS}

1. Introduction

2. Rationality problem for norm one tori

3. Strategy: flabby resolution of $G$-lattices

4. Proof of Theorem 1.5] and Theorem [1.6

5. Proof of Theorem 1.15 and Theorem 1.17

6. Proof of Theorem 1.18

7. Application 1: $R$-equivalence in algebraic $k$-tori

8. Application 2: Tamagawa number $\tau(T)$

9. Appendix: Computation of $H^{1}\left(G, J_{G / H}\right)$ for $G=\operatorname{Gal}(L / k)=n T m(n \leq 15)$

10. GAP algorithms

References

\section{INTRODUCTION}

Let $k$ be a field, $\bar{k}$ be a fixed separable closure of $k$ and $\mathcal{G}=\operatorname{Gal}(\bar{k} / k)$ be the absolute Galois group of $k$. Let $T$ be an algebraic $k$-torus, i.e. a group $k$-scheme with fiber product (base change) $T \times_{k} \bar{k}=T \times_{\text {Spec } k} \operatorname{Spec} \bar{k} \simeq\left(\mathbb{G}_{m, \bar{k}}\right)^{n}$; $k$-form of the split torus $\left(\mathbb{G}_{m}\right)^{n}$. Then there exists the minimal (canonical) finite Galois extension $K / k$ with Galois group $G=\operatorname{Gal}(K / k)$ such that $T$ splits over $K: T \times_{k} K \simeq\left(\mathbb{G}_{m, K}\right)^{n}$. It is also well-known that there is the duality between the category of $G$-lattices, i.e. finitely generated $\mathbb{Z}[G]$-modules which are $\mathbb{Z}$-free as abelian groups, and the category of algebraic $k$-tori which split over $K$ (see Ono Ono61, Section 1.2], Voskresenskii [Vos98, page 27, Example 6] and Knus, Merkurjev, Rost and Tignol [KMRT98, page 333, Proposition 20.17]). Indeed, if $T$ is an algebraic $k$-torus, then the character module $\widehat{T}=\operatorname{Hom}\left(T, \mathbb{G}_{m}\right)$ of $T$ may be regarded as a $G$-lattice. Let $X$ be a smooth $k$-compactification of $T$, i.e. smooth projective $k$-variety $X$ containing $T$ as a dense open subvariety, and $\bar{X}=X \times_{k} \bar{k}$. There exists such a smooth $k$-compactification of an algebraic $k$-torus $T$ over any field $k$ (due to Hironaka Hir64 for char $k=0$, see Colliot-Thélène, Harari and Skorobogatov [CTHS05, Corollaire 1] for any field $k$ ). A $\mathcal{G}$-lattice $P$ is said to be permutation if $P$ has a $\mathbb{Z}$-basis permuted by $\mathcal{G}$ and a $\mathcal{G}$-lattice $F$ is said to be flabby (resp. coflabby) if $\widehat{H}^{-1}(\mathcal{H}, F)=0$ (resp. $\left.H^{1}(\mathcal{H}, F)=0\right)$ for any closed subgroup $\mathcal{H} \leq \mathcal{G}$ where $\widehat{H}$ is the Tate cohomology.

2010 Mathematics Subject Classification. Primary 11E72, 12F20, 13A50, 14E08, 20C10, $20 \mathrm{G} 15$.

Key words and phrases. Algebraic tori, norm one tori, Hasse norm principle, weak approximation, rationality problem, flabby resolution.

This work was partially supported by JSPS KAKENHI Grant Numbers 16K05059, 19K03418, 20K03511. Parts of the work were finished when the first-named author and the third-named author were visiting the National Center for Theoretic Sciences (Taipei), whose support is gratefully acknowledged. 
Theorem 1.1 (Voskresenskii Vos69, Section 4, page 1213], Vos70, Section 3, page 7], see also [Vos98, Section 4.6], Kun07, Theorem 1.9], [Vos74 and [CT07, Theorem 5.1, page 19] for any field $k$ ). Let $k$ be a field and $\mathcal{G}=\operatorname{Gal}(\bar{k} / k)$. Let $T$ be an algebraic $k$-torus, $X$ be a smooth $k$-compactification of $T$ and $\bar{X}=X \times_{k} \bar{k}$. Then there exists an exact sequence of $\mathcal{G}$-lattices

$$
0 \rightarrow \widehat{T} \rightarrow \widehat{Q} \rightarrow \operatorname{Pic} \bar{X} \rightarrow 0
$$

where $\widehat{Q}$ is permutation and Pic $\bar{X}$ is flabby.

We have $H^{1}(k, \operatorname{Pic} \bar{X}) \simeq H^{1}\left(G, \operatorname{Pic} X_{K}\right)$ where $K$ is the splitting field of $T, G=\operatorname{Gal}(K / k)$ and $X_{K}=X \times_{k} K$. Hence Theorem 1.1 says that for $G$-lattices $M=\widehat{T}$ and $P=\widehat{Q}$, the exact sequence $0 \rightarrow M \rightarrow P \rightarrow \operatorname{Pic} X_{K} \rightarrow 0$ gives a flabby resolution of $M$ and the flabby class of $M$ is $[M]^{f l}=\left[\right.$ Pic $\left.X_{K}\right]$ as $G$-lattices (see Section 3 , cf. Endo and Miyata's theorem [EM75, Lemma 1.1] (= Theorem 3.4 in the present paper)).

Let $k$ be a global field, i.e. a number field (a finite extension of $\mathbb{Q}$ ) or a function field of an algebraic curve over $\mathbb{F}_{q}$ (a finite extension of $\mathbb{F}_{q}(t)$ ). Let $T$ be an algebraic $k$-torus and $T(k)$ be the group of $k$-rational points of $T$. Then $T(k)$ embeds into $\prod_{v \in V_{k}} T\left(k_{v}\right)$ by the diagonal map where $V_{k}$ is the set of all places of $k$ and $k_{v}$ is the completion of $k$ at $v$. Let $\overline{T(k)}$ be the closure of $T(k)$ in the product $\prod_{v \in V_{k}} T\left(k_{v}\right)$. The group

$$
A(T)=\left(\prod_{v \in V_{k}} T\left(k_{v}\right)\right) / \overline{T(k)}
$$

is called the kernel of the weak approximation of $T$. We say that $T$ has the weak approximation property if $A(T)=0$.

Let $E$ be a principal homogeneous space (= torsor) under $T$. Hasse principle holds for $E$ means that if $E$ has a $k_{v}$-rational point for all $k_{v}$, then $E$ has a $k$-rational point. The set $H^{1}(k, T)$ classifies all such torsors $E$ up to (non-unique) isomorphism. We define the Shafarevich-Tate group

$$
\amalg(T)=\operatorname{Ker}\left\{H^{1}(k, T) \stackrel{\text { res }}{\longrightarrow} \bigoplus_{v \in V_{k}} H^{1}\left(k_{v}, T\right)\right\} .
$$

Then Hasse principle holds for all torsors $E$ under $T$ if and only if $\amalg(T)=0$.

Theorem 1.2 (Voskresenskii Vos69, Theorem 5, page 1213], Vos70, Theorem 6, page 9], see also [Vos98, Section 11.6, Theorem, page 120]). Let $k$ be a global field, $T$ be an algebraic $k$-torus and $X$ be a smooth $k$-compactification of $T$. Then there exists an exact sequence

$$
0 \rightarrow A(T) \rightarrow H^{1}(k, \operatorname{Pic} \bar{X})^{\vee} \rightarrow \amalg(T) \rightarrow 0
$$

where $M^{\vee}=\operatorname{Hom}(M, \mathbb{Q} / \mathbb{Z})$ is the Pontryagin dual of $M$. Moreover, if $L$ is the splitting field of $T$ and $L / k$ is an unramified extension, then $A(T)=0$ and $H^{1}(k, \operatorname{Pic} \bar{X})^{\vee} \simeq \amalg(T)$.

For the last assertion, see $\operatorname{Vos} 98$, Theorem, page 120]. It follows that $H^{1}(k, \operatorname{Pic} \bar{X})=0$ if and only if $A(T)=0$ and $\amalg(T)=0$, i.e. $T$ has the weak approximation property and Hasse principle holds for all torsors $E$ under $T$. Theorem 1.2 was generalized to the case of linear algebraic groups by Sansuc [San81].

The norm one torus $R_{K / k}^{(1)}\left(\mathbb{G}_{m}\right)$ of $K / k$ is the kernel of the norm map $R_{K / k}\left(\mathbb{G}_{m}\right) \rightarrow \mathbb{G}_{m}$ where $R_{K / k}$ is the Weil restriction (see [Vos98, page 37, Section 3.12]). Such a torus $R_{K / k}^{(1)}\left(\mathbb{G}_{m}\right)$ is biregularly isomorphic to the norm hypersurface $f\left(x_{1}, \ldots, x_{n}\right)=1$ where $f \in k\left[x_{1}, \ldots, x_{n}\right]$ is the polynomial of total degree $n$ defined by the norm map $N_{K / k}: K^{\times} \rightarrow k^{\times}$. When $K / k$ is a finite Galois extension, we have that:

Theorem 1.3 (Voskresenskii [Vos70, Theorem 7], Colliot-Thélène and Sansuc [CTS77, Proposition 1]). Let $k$ be a field and $K / k$ be a finite Galois extension with Galois group $G=\operatorname{Gal}(K / k)$. Let $T=R_{K / k}^{(1)}\left(\mathbb{G}_{m}\right)$ be the norm one torus of $K / k$ and $X$ be a smooth $k$-compactification of $T$. Then $H^{1}\left(H, \operatorname{Pic} X_{K}\right) \simeq H^{3}(H, \mathbb{Z})$ for any subgroup $H$ of $G$. In particular, $H^{1}(k, \operatorname{Pic} \bar{X}) \simeq H^{1}\left(G, \operatorname{Pic} X_{K}\right) \simeq H^{3}(G, \mathbb{Z})$ which is isomorphic to the Schur multiplier $M(G)$ of $G$.

In other words, for $G$-lattice $J_{G}=\widehat{T}, H^{1}\left(H,\left[J_{G}\right]^{f l}\right) \simeq H^{3}(H, \mathbb{Z})$ for any subgroup $H$ of $G$ and $H^{1}\left(G,\left[J_{G}\right]^{f l}\right) \simeq$ $H^{3}(G, \mathbb{Z}) \simeq H^{2}(G, \mathbb{Q} / \mathbb{Z})$; the Schur multiplier of $G$. By the exact sequence $0 \rightarrow \mathbb{Z} \rightarrow \mathbb{Z}[G] \rightarrow J_{G} \rightarrow 0$, we also have $\delta: H^{1}\left(G, J_{G}\right) \simeq H^{2}(G, \mathbb{Z}) \simeq G^{a b} \simeq G /[G, G]$ where $\delta$ is the connecting homomorphism and $G^{a b}$ is the abelianization of $G$ (for details, see Section 2).

Let $K$ be a finitely generated field extension of a field $k$. A field $K$ is called rational over $k$ (or $k$-rational for short) if $K$ is purely transcendental over $k$, i.e. $K$ is isomorphic to $k\left(x_{1}, \ldots, x_{n}\right)$, the rational function field over $k$ with $n$ variables $x_{1}, \ldots, x_{n}$ for some integer $n . K$ is called stably $k$-rational if $K\left(y_{1}, \ldots, y_{m}\right)$ is $k$-rational for 
some algebraically independent elements $y_{1}, \ldots, y_{m}$ over $K$. Two fields $K$ and $K^{\prime}$ are called stably k-isomorphic if $K\left(y_{1}, \ldots, y_{m}\right) \simeq K^{\prime}\left(z_{1}, \ldots, z_{n}\right)$ over $k$ for some algebraically independent elements $y_{1}, \ldots, y_{m}$ over $K$ and $z_{1}, \ldots, z_{n}$ over $K^{\prime}$. When $k$ is an infinite field, $K$ is called retract $k$-rational if there is a $k$-algebra $R$ contained in $K$ such that (i) $K$ is the quotient field of $R$, and (ii) the identity map $1_{R}: R \rightarrow R$ factors through a localized polynomial ring over $k$, i.e. there is an element $f \in k\left[x_{1}, \ldots, x_{n}\right]$, which is the polynomial ring over $k$, and there are $k$-algebra homomorphisms $\varphi: R \rightarrow k\left[x_{1}, \ldots, x_{n}\right][1 / f]$ and $\psi: k\left[x_{1}, \ldots, x_{n}\right][1 / f] \rightarrow R$ satisfying $\psi \circ \varphi=1_{R}$ (cf. Sal84). $K$ is called $k$-unirational if $k \subset K \subset k\left(x_{1}, \ldots, x_{n}\right)$ for some integer $n$. It is not difficult to see that "k-rational" $\Rightarrow$ "stably $k$-rational" $\Rightarrow$ "retract $k$-rational" $\Rightarrow$ " $k$-unirational".

An algebraic $k$-torus $T$ is said to be $k$-rational (resp. stably $k$-rational, retract $k$-rational) if the function field $k(T)$ of $T$ is $k$-rational (resp. stably $k$-rational, retract $k$-rational).

Note that an algebraic $k$-torus $T$ is always $k$-unirational (see Vos98, page 40, Example 21]). Tori of dimension $n$ over $k$ correspond bijectively to the elements of the set $H^{1}\left(\mathcal{G}, \mathrm{GL}_{n}(\mathbb{Z})\right)$ where $\mathcal{G}=\operatorname{Gal}\left(k_{\mathrm{s}} / k\right)$ since $\operatorname{Aut}\left(\left(\mathbb{G}_{m}\right)^{n}\right)=$ $\mathrm{GL}_{n}(\mathbb{Z})$. The algebraic $k$-torus $T$ of dimension $n$ is determined uniquely by the integral representation $h: \mathcal{G} \rightarrow$ $\mathrm{GL}_{n}(\mathbb{Z})$ up to conjugacy, and the group $h(\mathcal{G})$ is a finite subgroup of $\mathrm{GL}_{n}(\mathbb{Z})$ (see [Vos98, page 57, Section 4.9])).

There are 2 (resp. 13, 73, 710, 6079) $\mathbb{Z}$-classes forming 2 (resp. 10, 32, 227, 955) $\mathbb{Q}$-classes in $\mathrm{GL}_{1}(\mathbb{Z})$ (resp. $\left.\mathrm{GL}_{2}(\mathbb{Z}), \mathrm{GL}_{3}(\mathbb{Z}), \mathrm{GL}_{4}(\mathbb{Z}), \mathrm{GL}_{5}(\mathbb{Z})\right)$. It is easy to see that all the 1-dimensional algebraic $k$-tori $T$, i.e. the trivial torus $\mathbb{G}_{m}$ and the norm one torus $R_{K / k}^{(1)}\left(\mathbb{G}_{m}\right)$ of $K / k$ with $[K: k]=2$, are $k$-rational. Voskresenskii Vos67. proved that all the 13 cases of 2 -dimensional algebraic $k$-tori, which correspond to $13 \mathbb{Z}$-conjugacy classes of finite subgroups of $\mathrm{GL}_{2}(\mathbb{Z})$, are $k$-rational. Note that whether all the 13 cases indeed occur or not depends on a base field $k$. The same applies for the numbers 15, 216 and 3003 in Theorems 1.4, 1.5 and 1.6 below. We also note that $T$ is retract $k$-rational $\Rightarrow H^{1}(k, \operatorname{Pic} \bar{X})=0$ and over global field $k, H^{1}(k, \operatorname{Pic} \bar{X})=0 \Rightarrow A(T) \simeq \amalg(T)=0$ (see Section 3 and also Manin [Man74, §30]).

Kunyavskii Kun90, solved the rationality problem for 3-dimensional algebraic $k$-tori. In the classification, there exist 73 cases of 3 -dimensional algebraic $k$-tori which correspond to $73 \mathbb{Z}$-conjugacy classes of finite subgroups of $\mathrm{GL}_{3}(\mathbb{Z})$, and 15 cases of them are not $k$-rational (resp. not stably $k$-rational, not retract $k$-rational). Using the classification, Kunyavskii Kun84 showed that only 2 cases of algebraic $k$-tori of dimension 3 satisfy the non-vanishing $H^{1}(k, \operatorname{Pic} \bar{X}) \neq 0$ among the 15 cases of non-rational $k$-tori. These two $k$-tori are norm one tori $T=R_{K / k}^{(1)}\left(\mathbb{G}_{m}\right)$ with $[K: k]=4$ :

Theorem 1.4 (Kunyavskii Kun84, Proposition 1]). Let $k$ be a field, $T$ be an algebraic $k$-torus of dimension 3 and $X$ be a smooth $k$-compactification of $T$. Then, among the (at most) 15 cases of non-rational algebraic $k$-tori $T$,

$$
H^{1}(k, \operatorname{Pic} \bar{X})= \begin{cases}\mathbb{Z} / 2 \mathbb{Z} & \text { if } T=R_{K_{1} / k}^{(1)}\left(\mathbb{G}_{m}\right) \text { or } R_{K_{2} / k}^{(1)}\left(\mathbb{G}_{m}\right) \\ 0 & \text { otherwise }\end{cases}
$$

where $K_{1} / k$ (resp. $\left.K_{2} / k\right)$ is a field extension of degree 4 whose Galois closure $L_{1} / k$ (resp. $L_{2} / k$ ) satisfies $\operatorname{Gal}\left(L_{1} / k\right) \simeq V_{4}$; the Klein four group (resp. $\operatorname{Gal}\left(L_{2} / k\right) \simeq A_{4}$; the alternating group of degree 4$)$. In particular, if $k$ is a global field, then $A(T) \simeq \amalg(T)=0$ except for $T=R_{K_{1} / k}^{(1)}\left(\mathbb{G}_{m}\right)$ and $T=R_{K_{2} / k}^{(1)}\left(\mathbb{G}_{m}\right)$.

Hoshi and Yamasaki HY17 classified stably/retract $k$-rational algebraic $k$-tori of dimensions 4 and 5 . In the classification, there exist 710 (resp. 6079) cases of 4-dimensional (resp. 5-dimensional) algebraic $k$-tori which correspond to 710 (resp. 6079) $\mathbb{Z}$-conjugacy classes of finite subgroups of $\mathrm{GL}_{4}(\mathbb{Z})$ (resp. $\mathrm{GL}_{5}(\mathbb{Z})$ ), and 216 (resp. $3003)$ cases of them are not retract $k$-rational.

The first main result (Theorem 1.5 and Theorem 1.6) of this paper is to classify the algebraic $k$-tori $T$ with non-vanishing $H^{1}(k, \operatorname{Pic} \bar{X}) \neq 0$ in dimensions 4 and 5 :

Theorem 1.5 (see Theorem 4.1 for the detailed statement). Let $k$ be a field, $T$ be an algebraic $k$-torus of dimension 4 and $X$ be a smooth $k$-compactification of $T$. Among the (at most) 216 cases of not retract rational algebraic $k$-tori $T$, there exist 2 (resp. 20, 194) cases of algebraic $k$-tori with $H^{1}(k, \operatorname{Pic} \bar{X}) \simeq(\mathbb{Z} / 2 \mathbb{Z})^{\oplus 2}($ resp. $\left.H^{1}(k, \operatorname{Pic} \bar{X}) \simeq \mathbb{Z} / 2 \mathbb{Z}, H^{1}(k, \operatorname{Pic} \bar{X})=0\right)$.

Theorem 1.6 (see Theorem 4.2 for the detailed statement). Let $k$ be a field, $T$ be an algebraic $k$-torus of dimension 5 and $X$ be a smooth $k$-compactification of $T$. Among the (at most) 3003 cases of not retract rational algebraic $k$-tori $T$, there exist 11 (resp. 263, 2729) cases of algebraic $k$-tori with $H^{1}(k, \operatorname{Pic} \bar{X}) \simeq(\mathbb{Z} / 2 \mathbb{Z})^{\oplus 2}$ (resp. $\left.H^{1}(k, \operatorname{Pic} \bar{X}) \simeq \mathbb{Z} / 2 \mathbb{Z}, H^{1}(k, \operatorname{Pic} \bar{X})=0\right)$.

Note that Hoshi and Yamasaki [HY17, Chapter 7] showed the vanishing $H^{1}(k, \operatorname{Pic} \bar{X}) \simeq H^{1}\left(G,[\widehat{T}]^{f l}\right)=0$ for any Bravais group $G$ of dimension $n \leq 6$ (see also [Vos83, Vos98, Section 8]). There exists 1 (resp. 5, 14, 64, 189, 841) Bravais group of dimension $n=1$ (resp. 2, 3, 4, 5, 6) (see [HY17, Example 4.16]). 
Let $G$ be a finite group and $M$ be a $G$-lattice. We define

$$
\amalg_{\omega}^{i}(G, M):=\operatorname{Ker}\left\{H^{i}(G, M) \stackrel{\text { res }}{\longrightarrow} \bigoplus_{g \in G} H^{i}(\langle g\rangle, M)\right\} \quad(i \geq 1) .
$$

The following is a theorem of Colliot-Thélène and Sansuc [CTS87:

Theorem 1.7 (Colliot-Thélène and Sansuc [CTS87, Proposition 9.5 (ii)], see also [San81, Proposition 9.8] and Vos98, page 98]). Let $k$ be a field with char $k=0$ and $K / k$ be a finite Galois extension with Galois group $G=\operatorname{Gal}(K / k)$. Let $T$ be an algebraic $k$-torus which splits over $K$ and $X$ be a smooth $k$-compactification of $T$. Then we have

$$
\amalg_{\omega}^{2}(G, \widehat{T}) \simeq H^{1}\left(G, \operatorname{Pic} X_{K}\right) \simeq \operatorname{Br}(X) / \operatorname{Br}(k)
$$

where $\operatorname{Br}(X)$ is the étale cohomological Brauer Group of $X$ (it is the same as the Azumaya-Brauer group of $X$ for such $X$, see [CTS87, page 199]).

In other words, for $G$-lattice $M=\widehat{T}$, we have $H^{1}(k, \operatorname{Pic} \bar{X}) \simeq H^{1}\left(G, \operatorname{Pic} X_{K}\right) \simeq H^{1}\left(G,[M]^{f l}\right) \simeq \amalg_{\omega}^{2}(G, M) \simeq$ $\operatorname{Br}(X) / \operatorname{Br}(k)$ (for the flabby class $[M]^{f l}$ of $M$, see Section 3 ). Hence Theorem 1.4 , Theorem 1.5 and Theorem 1.6 compute $H^{1}\left(G,[M]^{f l}\right) \simeq \amalg_{\omega}^{2}(G, M) \simeq \operatorname{Br}(X) / \operatorname{Br}(k)$ where $M=\widehat{T}$. We also see $\operatorname{Br}{ }_{n r}(k(X) / k)=\operatorname{Br}(X) \subset$ $\operatorname{Br}(k(X))$ (see Colliot-Thélène [CTS07, Theorem 5.11], Saltman [Sal99, Proposition 10.5]).

Let $k$ be a global field, $K / k$ be a finite extension and $\mathbb{A}_{K}^{\times}$be the idele group of $K$. We say that the Hasse norm principle holds for $K / k$ if $\left(N_{K / k}\left(\mathbb{A}_{K}^{\times}\right) \cap k^{\times}\right) / N_{K / k}\left(K^{\times}\right)=1$ where $N_{K / k}$ is the norm map.

Hasse [Has31, Satz, page 64] proved that the Hasse norm principle holds for any cyclic extension $K / k$ but does not hold for bicyclic extension $\mathbb{Q}(\sqrt{-39}, \sqrt{-3}) / \mathbb{Q}$. For Galois extensions $K / k$, Tate Tat67] gave the following theorem:

Theorem 1.8 (Tate [Tat67, page 198]). Let $k$ be a global field, $K / k$ be a finite Galois extension with Galois group $\operatorname{Gal}(K / k) \simeq G$. Let $V_{k}$ be the set of all places of $k$ and $G_{v}$ be the decomposition group of $G$ at $v \in V_{k}$. Then we have

$$
\left(N_{K / k}\left(\mathbb{A}_{K}^{\times}\right) \cap k^{\times}\right) / N_{K / k}\left(K^{\times}\right) \simeq \operatorname{Coker}\left\{\bigoplus_{v \in V_{k}} \widehat{H}^{-3}\left(G_{v}, \mathbb{Z}\right) \stackrel{\text { cores }}{\longrightarrow} \widehat{H}^{-3}(G, \mathbb{Z})\right\}
$$

where $\widehat{H}$ is the Tate cohomology. In particular, the Hasse norm principle holds for $K / k$ if and only if the restriction map $H^{3}(G, \mathbb{Z}) \stackrel{\text { res }}{\longrightarrow} \bigoplus_{v \in V_{k}} H^{3}\left(G_{v}, \mathbb{Z}\right)$ is injective.

Let $S_{n}$ (resp. $A_{n}, D_{n}, C_{n}$ ) be the symmetric (resp. the alternating, the dihedral, the cyclic) group of degree $n$ of order $n$ ! (resp. $n ! / 2,2 n, n$ ). Let $V_{4} \simeq C_{2} \times C_{2}$ be the Klein four group.

If $G \simeq C_{n}$ is cyclic, then $\widehat{H}^{-3}(G, \mathbb{Z}) \simeq H^{3}(G, \mathbb{Z}) \simeq H^{1}(G, \mathbb{Z})=0$ and hence the Hasse's original theorem follows. If there exists a place $v$ of $k$ such that $G_{v}=G$, then the Hasse norm principle also holds for $K / k$. For example, the Hasse norm principle holds for $K / k$ with $G \simeq V_{4}$ if and only if there exists a place $v$ of $k$ such that $G_{v}=V_{4}$ because $H^{3}\left(V_{4}, \mathbb{Z}\right) \simeq \mathbb{Z} / 2 \mathbb{Z}$ and $H^{3}\left(C_{2}, \mathbb{Z}\right)=0$. The Hasse norm principle holds for $K / k$ with $G \simeq\left(C_{2}\right)^{3}$ if and only if (i) there exists a place $v$ of $k$ such that $G_{v}=G$ or (ii) there exist places $v_{1}, v_{2}, v_{3}$ of $k$ such that $G_{v_{i}} \simeq V_{4}$ and $H^{3}(G, \mathbb{Z}) \stackrel{\text { res }}{\longrightarrow} H^{3}\left(G_{v_{1}}, \mathbb{Z}\right) \oplus H^{3}\left(G_{v_{2}}, \mathbb{Z}\right) \oplus H^{3}\left(G_{v_{3}}, \mathbb{Z}\right)$ is an isomorphism because $H^{3}(G, \mathbb{Z}) \simeq(\mathbb{Z} / 2 \mathbb{Z})^{\oplus 3}$ and $H^{3}\left(V_{4}, \mathbb{Z}\right) \simeq \mathbb{Z} / 2 \mathbb{Z}$

Ono Ono63] established the relationship between the Hasse norm principle for $K / k$ and the Hasse principle for all torsors under the norm one torus $R_{K / k}^{(1)}\left(\mathbb{G}_{m}\right)$ of $K / k$ :

Theorem 1.9 (Ono Ono63, page 70], see also Platonov [Pla82, page 44], Kunyavskii [Kun84, Remark 3], Platonov and Rapinchuk [PR94, page 307]). Let $k$ be a global field and $K / k$ be a finite extension. Then

$$
\amalg\left(R_{K / k}^{(1)}\left(\mathbb{G}_{m}\right)\right) \simeq\left(N_{K / k}\left(\mathbb{A}_{K}^{\times}\right) \cap k^{\times}\right) / N_{K / k}\left(K^{\times}\right) .
$$

In particular, $\amalg\left(R_{K / k}^{(1)}\left(\mathbb{G}_{m}\right)\right)=0$ if and only if the Hasse norm principle holds for $K / k$.

The Hasse norm principle for Galois extensions $K / k$ was investigated by Gerth Ger77, Ger78] and Gurak Gur78a, Gur78b, Gur80, (see also PR94, pages 308-309]), etc. Gurak Gur78a showed that the Hasse norm principle holds for Galois extension $K / k$ if all the Sylow subgroups of $\operatorname{Gal}(K / k)$ are cyclic. Note that this also follows from Theorem 1.9 and the retract $k$-rationality of $T=R_{K / k}^{(1)}\left(\mathbb{G}_{m}\right)$ due to Endo and Miyata EM75, Theorem 2.3].

However, for non-Galois extension $K / k$, very little is known about the Hasse norm principle. Bartels Bar81a (resp. [Bar81b] ) showed that the Hasse norm principle for $K / k$ holds when $[K: k]$ is prime $\left(\operatorname{resp}\right.$. Gal $\left.(L / k) \simeq D_{n}\right)$. 
The former case also follows from Theorem 1.9 and the retract $k$-rationality of $T=R_{K / k}^{(1)}\left(\mathbb{G}_{m}\right)$ due to ColliotThélène and Sansuc [CTS87, Proposition 9.1] (see Theorem 2.1).

Theorem 1.10 (Voskresenskii and Kunyavskii [VK84, see also Voskresenskii [Vos88, Theorem 4, Corollary]). Let $k$ be a number field, $K / k$ be a finite extension of degree $n$ and $L / k$ be the Galois closure of $K / k$ with $\operatorname{Gal}(L / k) \simeq S_{n}$; the symmetric group of degree $n$. Let $T=R_{K / k}^{(1)}\left(\mathbb{G}_{m}\right)$ be the norm one torus of $K / k$ and $X$ be a smooth $k$-compactification of $T$. Then $H^{1}\left(S_{n}, \operatorname{Pic} X_{L}\right)=0$. In particular, $T$ has the weak approximation property and the Hasse norm principle holds for $K / k$.

Theorem 1.11 (Macedo [Mac20]). Let $k$ be a number field, $K / k$ be a finite extension of degree $n \geq 5$ and $L / k$ be the Galois closure of $K / k$ with $\operatorname{Gal}(L / k) \simeq A_{n}$; the alternating group of degree $n \geq 5$. Let $T=R_{K / k}^{(1)}\left(\mathbb{G}_{m}\right)$ be the norm one torus of $K / k$. Then $\amalg_{\omega}^{2}\left(A_{n}, \widehat{T}\right)=0$. In particular, $T$ has the weak approximation property and the Hasse norm principle holds for $K / k$.

Remark 1.12. Applying Theorem 1.2 to $T=R_{K / k}^{(1)}\left(\mathbb{G}_{m}\right)$, it follows from Theorem 1.9 that $H^{1}(k$, Pic $\bar{X})=0$ if and only if $A(T)=0$ and $\amalg(T)=0$, i.e. $T$ has the weak approximation property and the Hasse norm principle holds for $K / k$. In the algebraic language, the latter condition $\amalg(T)=0$ means that for the corresponding norm hypersurface $f\left(x_{1}, \ldots, x_{n}\right)=b$, it has a $k$-rational point if and only if it has a $k_{v}$-rational point for any valuation $v$ of $k$ where $f \in k\left[x_{1}, \ldots, x_{n}\right]$ is the polynomial of total degree $n$ defined by the norm map $N_{K / k}: K^{\times} \rightarrow k^{\times}$ and $b \in k^{\times}$(see $\operatorname{Vos} 98$, Example 4, page 122]).

Let $n T m$ be the $m$-th transitive subgroup of $S_{n}$ up to conjugacy (see Butler and McKay [BM83], GAP]).

Let $k$ be a number field, $K / k$ be a field extension of degree $n$ and $L / k$ be the Galois closure of $K / k$ with $\operatorname{Gal}(L / k) \simeq G$. Then we may regard $G$ as the transitive subgroup $G=n T m \leq S_{n}$. Let $v$ be a place of $k$ and $G_{v}$ be the decomposition group of $G$ at $v$. Using Theorem 1.4 Kunyavskii Kun84 gave a necessary and sufficient condition for the Hasse norm principle for $n=4$ :

Theorem 1.13 (Kunyavskii Kun84, page 1899]). Let $k$ be a number field, $K / k$ be a field extension of degree 4 and $L / k$ be the Galois closure of $K / k$. Let $G=\operatorname{Gal}(L / k)=4 T m(1 \leq m \leq 5)$ be a transitive subgroup of $S_{4}$ and $H=\operatorname{Gal}(L / K)$ with $[G: H]=4$. Let $T=R_{K / k}^{(1)}\left(\mathbb{G}_{m}\right)$ be the norm one torus of $K / k$. Then $A(T) \simeq \amalg(T)=0$ except for $4 T 2 \simeq V_{4}$ and $4 T 4 \simeq A_{4}$. For $4 T 2 \simeq V_{4}$ and $4 T 4 \simeq A_{4}$, either (i) $A(T)=0$ and $\amalg(T) \simeq \mathbb{Z} / 2 \mathbb{Z}$ or $(\mathrm{ii})$ $A(T) \simeq \mathbb{Z} / 2 \mathbb{Z}$ and $\amalg(T)=0$, and the following conditions are equivalent:

(ii) $A(T) \simeq \mathbb{Z} / 2 \mathbb{Z}$ and $\amalg(T)=0$;

(iii) there exists a place $v$ of $k$ (which ramifies in $L$ ) such that $V_{4} \leq G_{v}$.

Drakokhrust and Platonov DP87] gave a necessary and sufficient condition for the Hasse norm principle for $n=6(G=6 \operatorname{Tm}(1 \leq m \leq 16))$ :

Theorem 1.14 (Drakokhrust and Platonov [DP87, Lemma 12, Proposition 6, Lemma 13]). Let $k$ be a number field, $K / k$ be a field extension of degree 6 and $L / k$ be the Galois closure of $K / k$. Let $G=\mathrm{Gal}(L / k)=6 T m$ $(1 \leq m \leq 16)$ be a transitive subgroup of $S_{6}$ and $H=\operatorname{Gal}(L / K)$ with $[G: H]=6$. Let $T=R_{K / k}^{(1)}\left(\mathbb{G}_{m}\right)$ be the norm one torus of $K / k$. Then $\amalg(T)=0$ except for $6 T 4 \simeq A_{4}$ and $6 T 12 \simeq A_{5}$. For $6 T 4 \simeq A_{4}$ and $6 T 12 \simeq A_{5}$, (i) $\amalg(T) \leq \mathbb{Z} / 2 \mathbb{Z}$; and (ii) $\amalg(T)=0$ if and only if there exists a place $v$ of $k$ (which ramifies in $L)$ such that $V_{4} \leq G_{v}$.

The number of transitive subgroups $n T m$ of $S_{n}(2 \leq n \leq 15)$ up to conjugacy is given as follows (see Butler and McKay [BM83] for $n \leq 11$, Royle Roy87 for $n=12$, Butler [But93] for $n=14,15$ and [GAP]):

\begin{tabular}{r|rrrrrrrrrrrrrr}
$n$ & 2 & 3 & 4 & 5 & 6 & 7 & 8 & 9 & 10 & 11 & 12 & 13 & 14 & 15 \\
\hline \# of $n T m$ & 1 & 2 & 5 & 5 & 16 & 7 & 50 & 34 & 45 & 8 & 301 & 9 & 63 & 104
\end{tabular}

The following theorem which is one of the main results of this paper classifies the norm one tori $T=R_{K / k}^{(1)}\left(\mathbb{G}_{m}\right)$ with non-vanishing $H^{1}(k, \operatorname{Pic} \bar{X}) \neq 0$ for $[K: k]=n \leq 15$ and $n \neq 12$.

Theorem 1.15. Let $2 \leq n \leq 15$ be an integer with $n \neq 12$. Let $k$ be a field, $K / k$ be a separable field extension of degree $n$ and $L / k$ be the Galois closure of $K / k$. Assume that $G=\operatorname{Gal}(L / k)=n T m$ is a transitive subgroup of $S_{n}$ and $H=\operatorname{Gal}(L / K)$ with $[G: H]=n$. Let $T=R_{K / k}^{(1)}\left(\mathbb{G}_{m}\right)$ be the norm one torus of K/k of dimension $n-1$ and $X$ be a smooth $k$-compactification of $T$. Then $H^{1}(k, \operatorname{Pic} \bar{X}) \neq 0$ if and only if $G$ is given as in Table 1 . In particular, if $k$ is a number field and $L / k$ is an unramified extension, then $A(T)=0$ and $H^{1}(k, \operatorname{Pic} \bar{X}) \simeq \amalg(T)$. 
Table 1: $H^{1}(k, \operatorname{Pic} \bar{X}) \simeq H^{1}\left(G,\left[J_{G / H}\right]^{f l}\right) \neq 0$ where $G=n T m$ with $2 \leq n \leq 15$ and $n \neq 12$

\begin{tabular}{lc}
$G$ & $H^{1}(k, \mathrm{Pic} \bar{X}) \simeq H^{1}\left(G,\left[J_{G / H}\right]^{f l}\right)$ \\
\hline $4 T 2 \simeq V_{4}$ & $\mathbb{Z} / 2 \mathbb{Z}$ \\
$4 T 4 \simeq A_{4}$ & $\mathbb{Z} / 2 \mathbb{Z}$ \\
\hline $6 T 4 \simeq A_{4}$ & $\mathbb{Z} / 2 \mathbb{Z}$ \\
$6 T 12 \simeq A_{5}$ & $\mathbb{Z} / 2 \mathbb{Z}$ \\
\hline $8 T 2 \simeq C_{4} \times C_{2}$ & $\mathbb{Z} / 2 \mathbb{Z}$ \\
$8 T 3 \simeq\left(C_{2}\right)^{3}$ & $(\mathbb{Z} / 2 \mathbb{Z})^{\oplus 3}$ \\
$8 T 4 \simeq D_{4}$ & $\mathbb{Z} / 2 \mathbb{Z}$ \\
$8 T 9 \simeq D_{4} \times C_{2}$ & $\mathbb{Z} / 2 \mathbb{Z}$ \\
$8 T 11 \simeq\left(C_{4} \times C_{2}\right) \rtimes C_{2}$ & $\mathbb{Z} / 2 \mathbb{Z}$ \\
$8 T 13 \simeq A_{4} \times C_{2}$ & $\mathbb{Z} / 2 \mathbb{Z}$ \\
$8 T 14 \simeq S_{4}$ & $\mathbb{Z} / 2 \mathbb{Z}$ \\
$8 T 15 \simeq C_{8} \rtimes V_{4}$ & $\mathbb{Z} / 2 \mathbb{Z}$ \\
$8 T 19 \simeq\left(C_{2}\right)^{3} \rtimes C_{4}$ & $\mathbb{Z} / 2 \mathbb{Z}$ \\
$8 T 21 \simeq\left(C_{2}\right)^{3} \rtimes C_{4}$ & $\mathbb{Z} / 2 \mathbb{Z}$ \\
$8 T 22 \simeq\left(C_{2}\right)^{3} \rtimes V_{4}$ & $\mathbb{Z} / 2 \mathbb{Z}$ \\
$8 T 31 \simeq\left(\left(C_{2}\right)^{4} \rtimes C_{2}\right) \rtimes C_{2}$ & $\mathbb{Z} / 2 \mathbb{Z}$ \\
$8 T 32 \simeq\left(\left(C_{2}\right)^{3} \rtimes V_{4}\right) \rtimes C_{3}$ & $\mathbb{Z} / 2 \mathbb{Z}$ \\
$8 T 37 \simeq \mathrm{PSL}_{3}\left(\mathbb{F}_{2}\right) \simeq \mathrm{PSL}_{2}\left(\mathbb{F}_{7}\right)$ & $\mathbb{Z} / 2 \mathbb{Z}$ \\
$8 T 38 \simeq\left(\left(\left(C_{2}\right)^{4} \rtimes C_{2}\right) \rtimes C_{2}\right) \rtimes C_{3}$ & $\mathbb{Z} / 2 \mathbb{Z}$ \\
\hline $9 T 2 \simeq\left(C_{3}\right)^{2}$ & $\mathbb{Z} / 3 \mathbb{Z}$ \\
$9 T 5 \simeq\left(C_{3}\right)^{2} \rtimes C_{2}$ & $\mathbb{Z} / 3 \mathbb{Z}$ \\
$9 T 7 \simeq\left(C_{3}\right)^{2} \rtimes C_{3}$ & $\mathbb{Z} / 3 \mathbb{Z}$ \\
$9 T 9 \simeq\left(C_{3}\right)^{2} \rtimes C_{4}$ & $\mathbb{Z} / 3 \mathbb{Z}$ \\
$9 T 11 \simeq\left(C_{3}\right)^{2} \rtimes C_{6}$ & $\mathbb{Z} / 3 \mathbb{Z}$ \\
$9 T 14 \simeq\left(C_{3}\right)^{2} \rtimes Q_{8}$ & $\mathbb{Z} / 3 \mathbb{Z}$ \\
$9 T 23 \simeq\left(\left(C_{3}\right)^{2} \rtimes Q_{8}\right) \rtimes C_{3}$ & $\mathbb{Z} / 3 \mathbb{Z}$ \\
\hline $10 T 7 \simeq A_{5}$ & $\mathbb{Z} / 2 \mathbb{Z}$ \\
$10 T 26 \simeq \mathrm{PSL}_{2}\left(\mathbb{F}_{9}\right) \simeq A_{6}$ & $\mathbb{Z} / 2 \mathbb{Z}$ \\
$10 T 32 \simeq S_{6}$ & $\mathbb{Z} / 2 \mathbb{Z}$ \\
\hline $14 T 30 \simeq \mathrm{PSL}_{2}\left(\mathbb{F}_{13}\right)$ & $\mathbb{Z} / 2 \mathbb{Z}$ \\
\hline $15 T 9 \simeq\left(C_{5}\right)^{2} \rtimes C_{3}$ & $\mathbb{Z} / 5 \mathbb{Z}$ \\
$15 T 14 \simeq\left(C_{5}\right)^{2} \rtimes S_{3}$ & $\mathbb{Z} / 5 \mathbb{Z}$ \\
\hline &
\end{tabular}

Remark 1.16. In Table 1, only the abelian groups of prime exponent $p$ appear as $H^{1}(k, \operatorname{Pic} \bar{X})$. However, we find that $H^{1}(k, \operatorname{Pic} \bar{X}) \simeq \mathbb{Z} / 4 \mathbb{Z}$ for $G=12 T 31 \simeq\left(C_{4}\right)^{2} \rtimes C_{3}$ and $G=12 T 57 \simeq\left(\left(C_{4} \times C_{2}\right) \rtimes C_{4}\right) \rtimes C_{3}$ by using the same technique as in the proof of Theorem 1.15

Additionally, by using the same method of Theorem 1.15, we obtain the vanishing $H^{1}(k, \operatorname{Pic} \bar{X})=0$ for the 5 Mathieu groups $M_{n} \leq S_{n}$ where $n=11,12,22,23,24$ (see Dixon and Mortimer [DM96. Chapter 6], Gorenstein, Lyons and Solomon [GLS98, Chapter 5] for the 5 Mathieu groups):

Theorem 1.17. Let $k$ be a field, $K / k$ be a separable field extension of degree $n$ and $L / k$ be the Galois closure of $K / k$. Assume that $G=\operatorname{Gal}(L / k)=M_{n} \leq S_{n}(n=11,12,22,23,24)$ is the Mathieu group of degree $n$ and $H=\operatorname{Gal}(L / K)$ with $[G: H]=n$. Let $T=R_{K / k}^{(1)}\left(\mathbb{G}_{m}\right)$ be the norm one torus of $K / k$ of dimension $n-1$ and $X$ be a smooth $k$-compactification of $T$. Then $H^{1}(k, \operatorname{Pic} \bar{X})=0$. In particular, if $k$ is a number field, then $A(T)=0$ and $\amalg(T)=0$.

Let $Z(G)$ be the center of a group $G,[G, G]$ be the commutator group of $G$ and $\operatorname{Syl}_{p}(G)$ be a $p$-Sylow subgroup of $G$ where $p$ is a prime. Let $\operatorname{Orb}_{G}(i)$ be the orbit of $1 \leq i \leq n$ under the action of $G \leq S_{n}$.

By Theorem 1.15, we obtain the following theorem which gives a necessary and sufficient condition for the Hasse norm principle for $K / k$ where $[K: k]=n \leq 15$ and $n \neq 12$. Note that a place $v$ of $k$ with non-cyclic decomposition group $G_{v}$ as in Theorem 1.18 must be ramified in $L$ because if $v$ is unramified, then $G_{v}$ is cyclic.

Theorem 1.18. Let $2 \leq n \leq 15$ be an integer with $n \neq 12$. Let $k$ be a number field, $K / k$ be a field extension of degree $n$ and $L / k$ be the Galois closure of $K / k$. Assume that $G=\operatorname{Gal}(L / k)=n T m$ is a transitive subgroup of $S_{n}, H=\operatorname{Gal}(L / K)$ with $[G: H]=n$ and $G_{v}$ is the decomposition group of $G$ at a place $v$ of $k$. Let 
$T=R_{K / k}^{(1)}\left(\mathbb{G}_{m}\right)$ be the norm one torus of $K / k$ of dimension $n-1$ and $X$ be a smooth $k$-compactification of T. Then $A(T) \simeq \amalg(T)=0$ except for the cases in Table 1. For the cases in Table 1 except for $G=8 T 3$, either (a) $A(T)=0$ and $\amalg(T) \simeq H^{1}(k, \operatorname{Pic} \bar{X})$ or $(\mathrm{b}) A(T) \simeq H^{1}(k, \operatorname{Pic} \bar{X})$ and $\amalg(T)=0$. For $G=8 T m$ $(m=9,11,15,19,22,32)$, we assume that $H$ is the stabilizer of one of the letters in $G$. Then a necessary and sufficient condition for $\amalg(T)=0$ is given as in Table 2.

Table 2: $\amalg(T)=0$ for $T=R_{K / k}^{(1)}\left(\mathbb{G}_{m}\right)$ and $G=\operatorname{Gal}(L / k)=n T m$ as in Table 1

\begin{tabular}{|c|c|}
\hline$G$ & $\amalg(T)=0$ if and only if there exists a place $v$ of $k$ such that \\
\hline $\begin{array}{l}4 T 2 \simeq V_{4} \\
4 T 4 \simeq A_{4}\end{array}$ & $\begin{aligned} V_{4} \leq G_{v} & \left(\text { Tate [Tat67] for } 4 T 2 \simeq V_{4}\right) \\
& \left(\text { Kunyavskii } \text { Kun84] for } 4 T 4 \simeq A_{4}\right)\end{aligned}$ \\
\hline $\begin{array}{l}6 T 4 \simeq A_{4} \\
6 T 12 \simeq A_{5}\end{array}$ & $V_{4} \leq G_{v}($ Drakokhrust and Platonov [DP87] $)$ \\
\hline $8 T 3 \simeq\left(C_{2}\right)^{3}$ & see the second paragraph after Theorem[1.8 (Tate [Tat67]) \\
\hline $\begin{array}{l}8 T 4 \simeq D_{4} \\
8 T 13 \simeq A_{4} \times C_{2} \\
8 T 14 \simeq S_{4} \\
8 T 37 \simeq \mathrm{PSL}_{2}\left(\mathbb{F}_{7}\right)\end{array}$ & $V_{4} \leq G_{v}\left(\right.$ Tate [Tat67] for $\left.8 T 4 \simeq D_{4}\right)$ \\
\hline $\begin{array}{l}8 T 2 \simeq C_{4} \times C_{2} \\
8 T 21 \simeq\left(C_{2}\right)^{3} \rtimes C_{4}\end{array}$ & $G_{v}=G\left(\right.$ Tate [Tat67] for $\left.8 T 2 \simeq C_{4} \times C_{2}\right)$ \\
\hline $\begin{array}{l}8 T 31 \simeq\left(C_{2}\right)^{4} \rtimes V_{4} \\
8 T 38 \simeq 8 T 31 \rtimes C_{3}\end{array}$ & $\begin{array}{l}\text { (i) } V_{4} \leq G_{v} \text { where } V_{4} \cap\left[\operatorname{Syl}_{2}(G), \operatorname{Syl}_{2}(G)\right]=1 \text { with } \operatorname{Syl}_{2}(G) \triangleleft G \\
\left.\text { (equivalently, }\left|\operatorname{Orb}_{V_{4}}(i)\right|=4 \text { for any } 1 \leq i \leq 8 \text { and } V_{4} \cap Z(G)=1\right) \text {, (ii) } \\
C_{4} \times C_{2} \leq G_{v} \text { where }\left(C_{4} \times C_{2}\right) \cap\left[\operatorname{Syl}_{2}(G), \operatorname{Syl}_{2}(G)\right] \simeq C_{2} \text { (equivalently, } \\
\left.C_{4} \times C_{2} \text { is transitive in } S_{8}\right) \text { or (iii) }\left(C_{2}\right)^{3} \rtimes C_{4} \leq G_{v}\end{array}$ \\
\hline $8 T 9 \simeq D_{4} \times C_{2}$ & $\begin{array}{l}\text { (i) } V_{4} \leq G_{v} \text { where }\left|\operatorname{Orb}_{V_{4}}(i)\right|=4 \text { for any } 1 \leq i \leq 8 \text { and } V_{4} \cap[G, G]=1 \text {; } \\
\text { or (ii) } C_{4} \times C_{2} \leq G_{v}\end{array}$ \\
\hline $8 T 11 \simeq Q_{8} \rtimes C_{2}$ & $C_{4} \times C_{2} \leq G_{v}$ where $C_{4} \times C_{2}$ is transitive in $S_{8}$ \\
\hline $8 T 15 \simeq C_{8} \rtimes V_{4}$ & $\begin{array}{l}\text { (i) } V_{4} \leq G_{v} \text { where }\left|\operatorname{Orb}_{V_{4}}(i)\right|=2 \text { for any } 1 \leq i \leq 8 \text { and } V_{4} \cap[G, G]=1 \\
\text { (equivalently, }\left|\operatorname{Orb}_{V_{4}}(i)\right|=2 \text { for any } 1 \leq i \leq 8 \text { and } V_{4} \text { is not in } A_{8} \text { ) } \\
\text { or (ii) } C_{4} \times C_{2} \leq G_{v} \text { where }\left(C_{4} \times C_{2}\right) \cap[G, G] \simeq C_{2} \text { (equivalently, } \\
C_{4} \times C_{2} \text { is transitive in } S_{8} \text { ) }\end{array}$ \\
\hline $8 T 19 \simeq\left(C_{2}\right)^{3} \rtimes C_{4}$ & $\begin{array}{l}\text { (i) } V_{4} \leq G_{v} \text { where } V_{4} \cap Z(G)=1 \text { and } V_{4} \cap Z^{2}(G) \simeq C_{2} \text { with the upper } \\
\text { central series } 1 \leq Z(G) \leq Z^{2}(G) \leq G \text { (equivalently, }\left|\operatorname{Orb}_{V_{4}}(i)\right|=4 \\
\text { for any } 1 \leq i \leq 8 \text { and } V_{4} \cap Z(G)=1 \text { ); or (ii) } C_{4} \times C_{2} \leq G_{v} \text { where } \\
C_{4} \times C_{2} \text { is not transitive in } S_{8} \text { or }[G, G] \leq C_{4} \times C_{2}\end{array}$ \\
\hline $\begin{array}{l}8 T 22 \simeq\left(C_{2}\right)^{3} \rtimes V_{4} \\
8 T 32 \simeq 8 T 22 \rtimes C_{3}\end{array}$ & $\begin{array}{l}\text { (i) } V_{4} \leq G_{v} \text { where } \mid \text { Orb }_{V_{4}}(i) \mid=4 \text { for any } 1 \leq i \leq 8 \text { and } V_{4} \cap Z(G)=1 \\
\text { or (ii) } C_{4} \times C_{2} \leq G_{v} \text { where } C_{4} \times C_{2} \text { is transitive in } S_{8}\end{array}$ \\
\hline $\begin{array}{l}9 T 2 \simeq\left(C_{3}\right)^{2} \\
9 T 5 \simeq\left(C_{3}\right)^{2} \rtimes C_{2} \\
9 T 7 \simeq\left(C_{3}\right)^{2} \rtimes C_{3} \\
9 T 9 \simeq\left(C_{3}\right)^{2} \rtimes C_{4} \\
9 T 11 \simeq\left(C_{3}\right)^{2} \rtimes C_{6} \\
9 T 14 \simeq\left(C_{3}\right)^{2} \rtimes Q_{8} \\
9 T 23 \simeq 9 T 14 \rtimes C_{3}\end{array}$ & $\left(C_{3}\right)^{2} \leq G_{v}\left(\right.$ Tate [Tat67] for $\left.9 T 2 \simeq\left(C_{3}\right)^{2}\right)$ \\
\hline $\begin{array}{l}10 T 7 \simeq A_{5} \\
10 T 26 \simeq \mathrm{PSL}_{2}\left(\mathbb{F}_{9}\right)\end{array}$ & $\begin{array}{l}V_{4} \leq G_{v} \\
D_{4} \leq G_{v}\end{array}$ \\
\hline $10 T 32 \simeq S_{6}$ & $\begin{array}{l}\text { (i) } V_{4} \leq G_{v} \text { where } N_{\widetilde{G}}\left(V_{4}\right) \simeq C_{8} \rtimes\left(C_{2} \times C_{2}\right) \text { for the normalizer } N_{\widetilde{G}}\left(V_{4}\right) \\
\text { of } V_{4} \text { in } \widetilde{G} \text { with the normalizer } \widetilde{G}=N_{S_{10}}(G) \simeq \operatorname{Aut}(G) \text { of } G \text { in } S_{10} \\
\text { (equivalently, }\left|\operatorname{Orb}_{V_{4}}(i)\right|=2 \text { for any } 1 \leq i \leq 10 \text { ) or (ii) } D_{4} \leq G_{v} \\
\text { where } D_{4} \leq[G, G] \simeq A_{6}\end{array}$ \\
\hline $14 T 30 \simeq \mathrm{PSL}_{2}\left(\mathbb{F}_{13}\right)$ & $V_{4} \leq G_{v}$ \\
\hline $\begin{array}{l}15 T 9 \simeq\left(C_{5}\right)^{2} \rtimes C_{3} \\
15 T 14 \simeq\left(C_{5}\right)^{2} \rtimes S_{3}\end{array}$ & $\left(C_{5}\right)^{2} \leq G_{v}$ \\
\hline
\end{tabular}


We organize this paper as follows. In Section 2, we prepare some basic definitions and known results about the rationality problem for norm one tori. In Section 3 , we recall our basic tool "flabby resolution of $G$-lattices" to investigate algebraic $k$-tori. In Section 4, we give the proof of Theorem 1.5 and Theorem 1.6. In Section 5, the proofs of Theorem 1.15] and Theorem 1.17 are given. In Section [6] we prove Theorem 1.18 by using Drakokhrust and Platonov's method for the Hasse norm principle for $K / k$. In Section 7 we will give an application of Theorem 1.5. Theorem 1.6 and Theorem 1.15 to obtain the group $T(k) / R$ of $R$-equivalence classes over a local field $k$ via the formula of Colliot-Thélène and Sansuc. In Section 8, we also give an application of Theorem[1.5. Theorem[1.6 and Theorem 1.18 to evaluate the Tamagawa number $\tau(T)$ over a number field $k$ via Ono's formula. In Section 9 , we will give GAP computations of $H^{1}\left(G, J_{G / H}\right)$ as the appendix of this paper. GAP algorithms will be given in Section 10 which are also available from https ://www . math.kyoto-u. ac.jp/〜yamasaki/Algorithm/Norm1ToriHNP.

Acknowledgments. We would like to thank Ming-chang Kang, Shizuo Endo and Boris Kunyavskii for giving us useful and valuable comments. We also thank the referees for very careful reading of the manuscript. This paper is greatly improved by their helpful comments and suggestions. In particular, one of them tell us the recent papers Macedo and Newton [MN] and Macedo [Mac] as a convergence of interests. It may be interesting to compare this paper with them.

\section{RATIONALITY PROBLEM FOR NORM ONE TORI}

Let $k$ be a field, $K / k$ be a separable field extension of degree $n$ and $L / k$ be the Galois closure of $K / k$. Let $G=\operatorname{Gal}(L / k)$ and $H=\operatorname{Gal}(L / K)$ with $[G: H]=n$. The Galois group $G$ may be regarded as a transitive subgroup of the symmetric group $S_{n}$ of degree $n$ via an injection $G \rightarrow S_{n}$ which is derived from the action of $G$ on the left cosets $\left\{g_{1} H, \ldots, g_{n} H\right\}$ by $g\left(g_{i} H\right)=\left(g g_{i}\right) H$ for any $g \in G$ and we may assume that $H$ is the stabilizer of one of the letters in $G$, i.e. $L=k\left(\theta_{1}, \ldots, \theta_{n}\right)$ and $K=k\left(\theta_{i}\right)$ for some $1 \leq i \leq n$. The norm one torus $R_{K / k}^{(1)}\left(\mathbb{G}_{m}\right)$ has the Chevalley module $J_{G / H}$ as its character module where $J_{G / H}=\left(I_{G / H}\right)^{\circ}=\operatorname{Hom}_{\mathbb{Z}}\left(I_{G / H}, \mathbb{Z}\right)$ is the dual lattice of $I_{G / H}=\operatorname{Ker} \varepsilon$ and $\varepsilon: \mathbb{Z}[G / H] \rightarrow \mathbb{Z}$ is the augmentation map (see [Vos98, Section 4.8]). We have the exact sequence

$$
0 \rightarrow \mathbb{Z} \rightarrow \mathbb{Z}[G / H] \rightarrow J_{G / H} \rightarrow 0
$$

and $\operatorname{rank} J_{G / H}=n-1$. Write $J_{G / H}=\oplus_{1 \leq i \leq n-1} \mathbb{Z} u_{i}$. We define the action of $G$ on $L\left(x_{1}, \ldots, x_{n-1}\right)$ by $\sigma\left(x_{i}\right)=\prod_{j=1}^{n} x_{j}^{a_{i, j}}(1 \leq i \leq n)$ for any $\sigma \in G$, when $\sigma\left(u_{i}\right)=\sum_{j=1}^{n} a_{i, j} u_{j}\left(a_{i, j} \in \mathbb{Z}\right)$. Then the invariant field $L\left(x_{1}, \ldots, x_{n-1}\right)^{G}$ may be identified with the function field of the norm one torus $R_{K / k}^{(1)}\left(\mathbb{G}_{m}\right)$ (see [EM75, Section 1]).

Let $T=R_{K / k}^{(1)}\left(\mathbb{G}_{m}\right)$ be the norm one torus of $K / k$. The rationality problem for norm one tori is investigated by [EM75, CTS77, Hür84, CTS87, LeB95, CK00, LL00, [Flo, End11, [HY17, [HY21, HHY20.

Theorem 2.1 (Colliot-Thélène and Sansuc [CTS87, Proposition 9.1], [LeB95, Theorem 3.1], [CK00, Proposition 0.2], [LL00, Endo [End11, Theorem 4.1], see also [End11, Remark 4.2 and Theorem 4.3]). Let $K / k$ be a nonGalois separable field extension of degree $n$ and $L / k$ be the Galois closure of $K / k$. Assume that $\operatorname{Gal}(L / k)=S_{n}$, $n \geq 3$, and $\operatorname{Gal}(L / K)=S_{n-1}$ is the stabilizer of one of the letters in $S_{n}$. Then we have:

(i) $R_{K / k}^{(1)}\left(\mathbb{G}_{m}\right)$ is retract $k$-rational if and only if $n$ is a prime;

(ii) $R_{K / k}^{(1)}\left(\mathbb{G}_{m}\right)$ is (stably) $k$-rational if and only if $n=3$.

Theorem 2.2 (Endo [End11, Theorem 4.4], Hoshi and Yamasaki [HY17, Corollary 1.11]). Let $K / k$ be a nonGalois separable field extension of degree $n$ and $L / k$ be the Galois closure of $K / k$. Assume that $\operatorname{Gal}(L / k)=A_{n}$, $n \geq 4$, and $\operatorname{Gal}(L / K)=A_{n-1}$ is the stabilizer of one of the letters in $A_{n}$. Then we have:

(i) $R_{K / k}^{(1)}\left(\mathbb{G}_{m}\right)$ is retract $k$-rational if and only if $n$ is a prime.

(ii) $R_{K / k}^{(1)}\left(\mathbb{G}_{m}\right)$ is stably $k$-rational if and only if $n=5$.

A necessary and sufficient condition for the classification of stably/retract rational norm one tori $T=$ $R_{K / k}^{(1)}\left(\mathbb{G}_{m}\right)$ with $[K: k]=n \leq 15$, but with one exception $G=9 T 27 \simeq \mathrm{PSL}_{2}\left(\mathbb{F}_{8}\right)$ for the stable rationality, was given in Hoshi and Yamasaki [HY21] (for the case $n$ is a prime number or the case $n \leq 10$ ) and Hasegawa, Hoshi and Yamasaki HHY20] (for $n=12,14,15$ ).

\section{Strategy: flabby Resolution of $G$-LAtTices}

We recall some basic facts of the theory of flabby (flasque) G-lattices (see [CTS77, Swa83, Vos98, Chapter 2], [Lor05, Chapter 2], Swa10]). Recall also that we may take the $G$-lattice $\widehat{T}$ for an algebraic $k$-torus $T$ (see Section 11). 
Definition 3.1. Let $G$ be a finite group and $M$ be a $G$-lattice (i.e. finitely generated $\mathbb{Z}[G]$-module which is $\mathbb{Z}$-free as an abelian group).

(i) $M$ is called a permutation $G$-lattice if $M$ has a $\mathbb{Z}$-basis permuted by $G$, i.e. $M \simeq \oplus_{1 \leq i \leq m} \mathbb{Z}\left[G / H_{i}\right]$ for some subgroups $H_{1}, \ldots, H_{m}$ of $G$.

(ii) $M$ is called a stably permutation $G$-lattice if $M \oplus P \simeq P^{\prime}$ for some permutation $G$-lattices $P$ and $P^{\prime}$.

(iii) $M$ is called invertible (or permutation projective) if it is a direct summand of a permutation $G$-lattice, i.e. $P \simeq M \oplus M^{\prime}$ for some permutation $G$-lattice $P$ and a $G$-lattice $M^{\prime}$.

(iv) $M$ is called flabby (or flasque) if $\widehat{H}^{-1}(H, M)=0$ for any subgroup $H$ of $G$ where $\widehat{H}$ is the Tate cohomology. (v) $M$ is called coflabby (or coflasque) if $H^{1}(H, M)=0$ for any subgroup $H$ of $G$.

Definition 3.2 (see [EM75, Section 1], Vos98, Section 4.7]). Let $\mathcal{C}(G)$ be the category of all $G$-lattices. Let $\mathcal{S}(G)$ be the full subcategory of $\mathcal{C}(G)$ of all permutation $G$-lattices and $\mathcal{D}(G)$ be the full subcategory of $\mathcal{C}(G)$ of all invertible $G$-lattices. Let

$$
\mathcal{H}^{i}(G)=\left\{M \in \mathcal{C}(G) \mid \widehat{H}^{i}(H, M)=0 \text { for any } H \leq G\right\}(i= \pm 1)
$$

be the class of " $\widehat{H}^{i}$-vanish" $G$-lattices where $\widehat{H}^{i}$ is the Tate cohomology. Then we have the inclusions $\mathcal{S}(G) \subset$ $\mathcal{D}(G) \subset \mathcal{H}^{i}(G) \subset \mathcal{C}(G)(i= \pm 1)$.

Definition 3.3. We say that two $G$-lattices $M_{1}$ and $M_{2}$ are similar if there exist permutation $G$-lattices $P_{1}$ and $P_{2}$ such that $M_{1} \oplus P_{1} \simeq M_{2} \oplus P_{2}$. We denote the similarity class of $M$ by $[M]$. The set of similarity classes $\mathcal{C}(G) / \mathcal{S}(G)$ becomes a commutative monoid (with respect to the sum $\left[M_{1}\right]+\left[M_{2}\right]:=\left[M_{1} \oplus M_{2}\right]$ and the zero $0=[P]$ where $P \in \mathcal{S}(G))$.

Theorem 3.4 (Endo and Miyata EM75, Lemma 1.1], Colliot-Thélène and Sansuc [CTS77, Lemma 3], see also Swa83, Lemma 8.5], Lor05, Lemma 2.6.1]). For any G-lattice $M$, there exists a short exact sequence of $G$-lattices $0 \rightarrow M \rightarrow P \rightarrow F \rightarrow 0$ where $P$ is permutation and $F$ is flabby.

Definition 3.5. The exact sequence $0 \rightarrow M \rightarrow P \rightarrow F \rightarrow 0$ as in Theorem 3.4 is called a flabby resolution of the $G$-lattice $M . \rho_{G}(M)=[F] \in \mathcal{C}(G) / \mathcal{S}(G)$ is called the flabby class of $M$, denoted by $[M]^{f l}=[F]$. Note that $[M]^{f l}$ is well-defined: if $[M]=\left[M^{\prime}\right],[M]^{f l}=[F]$ and $\left[M^{\prime}\right]^{f l}=\left[F^{\prime}\right]$ then $F \oplus P_{1} \simeq F^{\prime} \oplus P_{2}$ for some permutation $G$-lattices $P_{1}$ and $P_{2}$, and therefore $[F]=\left[F^{\prime}\right]$ (cf. [Swa83, Lemma 8.7]). We say that $[M]^{f l}$ is invertible if $[M]^{f l}=[E]$ for some invertible $G$-lattice $E$.

For $G$-lattice $M$, it is not difficult to see

$$
\begin{array}{ccc}
\text { permutation } \Rightarrow \text { stably permutation } & \Rightarrow \text { invertible } \Rightarrow \text { flabby and coflabby } \\
\Downarrow & \Downarrow \\
{[M]^{f l}=0} & \Rightarrow & {[M]^{f l} \text { is invertible. }}
\end{array}
$$

The above implications in each step cannot be reversed (see, for example, [HY17, Section 1]).

Let $T$ be an algebraic $k$-torus and $\widehat{T}=\operatorname{Hom}\left(T, \mathbb{G}_{m}\right)$ be the character module of $T$. Then $\widehat{T}$ becomes a $G$ lattice where $G=\operatorname{Gal}(L / k)$ is the Galois group of $L / k$ and $L$ is the minimal splitting field of $T$. The flabby class $\rho_{G}(\widehat{T})=[\widehat{T}]^{f l}$ plays crucial role in the rationality problem for $T$ as follows (see Voskresenskii's fundamental book Vos98, Section 4.6] and Kunyavskii [Kun07, see also e.g. Swan Swa83, Kunyavskii Kun90, Section 2], Lemire, Popov and Reichstein [LPR06, Section 2], Kang [Kan12, Yamasaki [Yam12, Hoshi and Yamasaki [HY17]):

Theorem 3.6 (Endo and Miyata EM73, Voskresenskii Vos74, Saltman [Sal84]). Let $T$ and $T^{\prime}$ be algebraic $k$-tori with the same minimal splitting field $L$. Then we have:

(i) (Endo and Miyata [EM73, Theorem 1.6]) $[\widehat{T}]^{f l}=0$ if and only if $T$ is stably $k$-rational;

(ii) (Voskresenskii Vos74, Theorem 2]) $[\widehat{T}]^{f l}=\left[\widehat{T}^{\prime}\right]^{f l}$ if and only if $T$ and $T^{\prime}$ are stably $k$-isomorphic;

(iii) (Saltman Sal84, Theorem 3.14]) $[\widehat{T}]^{f l}$ is invertible if and only if $T$ is retract $k$-rational.

For norm one tori $T=R_{K / k}^{(1)}\left(\mathbb{G}_{m}\right)$, recall that $\widehat{T}=J_{G / H}$. Hence we have

$$
\left[J_{G / H}\right]^{f l}=0 \Rightarrow\left[J_{G / H}\right]^{f l} \text { is invertible } \Rightarrow H^{1}\left(G,\left[J_{G / H}\right]^{f l}\right)=0 \Rightarrow A(T)=0 \text { and } \amalg(T)=0
$$

where the last implication holds over a global field $k$ (see also Colliot-Thélène and Sansuc [CTS77, page 29]). The last conditions mean that $T$ has the weak approximation property and the Hasse norm principle holds for $K / k$ (see Section (1). In particular, it follows from Theorem 2.1 that $H^{1}\left(G,\left[J_{G / H}\right]^{f l}\right)=0$ and hence $A(T)=0$ and $\amalg(T)=0$ when $G=p T m \leq S_{p}$ is a transitive subgroup of $S_{p}$ of prime degree $p$ and $H \leq G$ with $[G: H]=p$ (see [HY17, Lemma 2.17] and also the first paragraph of Section [5). 


\section{Proof of Theorem [1.5] And Theorem 1.6}

We will give the proof of Theorem 4.1 and Theorem 4.2 which are detailed statements of Theorem 1.5 and Theorem 1.6 respectively:

Theorem 4.1. Let $k$ be a field, $T$ be an algebraic $k$-torus of dimension 4 and $X$ be a smooth $k$-compactification of $T$. Among the (at most) 216 cases of not retract rational algebraic $k$-tori $T$, there exist 2 (resp. 20, 194) cases of algebraic $k$-tori with $H^{1}(k, \operatorname{Pic} \bar{X}) \simeq(\mathbb{Z} / 2 \mathbb{Z})^{\oplus 2}\left(\right.$ resp. $\left.H^{1}(k, \operatorname{Pic} \bar{X}) \simeq \mathbb{Z} / 2 \mathbb{Z}, H^{1}(k, \operatorname{Pic} \bar{X})=0\right)$. Moreover, for the character module $\widehat{T} \simeq M_{G}$ of $T$ with $H^{1}(k, \operatorname{Pic} \bar{X}) \simeq H^{1}\left(G,\left[M_{G}\right]^{f l}\right)$, we have

(i) $H^{1}\left(G,\left[M_{G}\right]^{f l}\right) \simeq(\mathbb{Z} / 2 \mathbb{Z})^{\oplus 2}$ if and only if the GAP ID of $G$ is one of $(4,32,1,2)$ and $(4,33,3,1)$ where $M_{G}$ is an indecomposable $G$-lattice of rank 4 and $G$ is isomorphic to $Q_{8}$ and $\mathrm{SL}_{2}\left(\mathbb{F}_{3}\right)$ respectively;

(ii) $H^{1}\left(G,\left[M_{G}\right]^{f l}\right) \simeq \mathbb{Z} / 2 \mathbb{Z}$ if and only if

(ii-1) the GAP ID of $G$ is one of $(4,5,1,12),(4,5,2,8),(4,6,2,10),(4,12,2,6),(4,12,4,12),(4,12,5,10)$, $(4,18,1,3),(4,18,4,4),(4,32,2,2),(4,32,3,2),(4,32,4,2),(4,32,6,2),(4,33,5,1),(4,33,6,1),(4,33,9,1)$ where $M_{G}$ is an indecomposable $G$-lattice of rank 4 and $G$ is isomorphic to $V_{4},\left(C_{2}\right)^{3},\left(C_{2}\right)^{3}, C_{4} \times C_{2}, D_{4}, C_{2} \times D_{4}$, $C_{4} \times C_{2}, C_{2} \times D_{4}, Q_{16}, Q D_{8},\left(C_{4} \times C_{2}\right) \rtimes C_{2}, C_{8} \rtimes V_{4},\left(\left(C_{4} \times C_{2}\right) \rtimes C_{2}\right) \rtimes C_{3}, \mathrm{GL}_{2}\left(\mathbb{F}_{3}\right), \mathrm{GL}_{2}\left(\mathbb{F}_{3}\right) \rtimes C_{2}$ respectively; or

(ii-2) the GAP ID of $G$ is one of $(4,4,3,6),(4,5,1,9),(4,6,2,9),(4,24,1,5),(4,25,2,4)$ where $M_{G}$ is a decomposable $G$-lattice of rank $4=3+1$ and $G$ is isomorphic to $V_{4}, V_{4},\left(C_{2}\right)^{3}, A_{4}, C_{2} \times A_{4}$ respectively.

Proof. It follows from [HY17, Theorem 1.9] that among the 710 cases of 4 -dimensional algebraic $k$-tori, there exist 216 cases of algebraic $k$-tori which are not retract $k$-rational. Because if $T$ is retract $k$-rational, then $H^{1}(k, \operatorname{Pic} \bar{X}) \simeq H^{1}\left(G,\left[M_{G}\right]^{f l}\right)=0$, we should check only the 216 cases. The GAP IDs of such 216 groups $G \leq \mathrm{GL}_{4}(\mathbb{Z})$ with $\left[M_{G}\right]^{f l}$ is not invertible, are given in [HY17, Tables 3, 4] (see [HY17, Chapter 3] for the explanation of GAP ID). They are also given in [HY17, Example 10.1] as the lists N4 (resp. N31) when $M_{G}$ is indecomposable (resp. decomposable with rank $4=3+1$ ) and available from

https://www.math.kyoto-u.ac.jp/ yamasaki/Algorithm/MultInvField/NonInv.dat.

Then we apply the function FlabbyResolutionLowRank $(G)$. actionF (see also HHY20, Algorithm 4.1]) which returns a suitable flabby class $F$ of $M_{G}\left([F]=\left[M_{G}\right]^{f l}\right)$ with low rank by using the backtracking techniques. The function $\mathrm{H} 1$ may compute the group $H^{1}(G, F)$ (see Example 4.4). The related functions are available from https ://www.math.kyoto-u.ac.jp/ yamasaki/Algorithm/RatProbNorm1Tori/.

Theorem 4.2. Let $k$ be a field, $T$ be an algebraic $k$-torus of dimension 5 and $X$ be a smooth $k$-compactification of T. Among the (at most) 3003 cases of not retract rational algebraic $k$-tori $T$, there exist 11 (resp. 263, 2729) cases of algebraic $k$-tori with $H^{1}(k, \operatorname{Pic} \bar{X}) \simeq(\mathbb{Z} / 2 \mathbb{Z})^{\oplus 2}\left(\right.$ resp. $\left.H^{1}(k, \operatorname{Pic} \bar{X}) \simeq \mathbb{Z} / 2 \mathbb{Z}, H^{1}(k, \operatorname{Pic} \bar{X})=0\right)$. Moreover, for the character module $\widehat{T} \simeq M_{G}$ of $T$ with $H^{1}(k, \operatorname{Pic} \bar{X}) \simeq H^{1}\left(G,\left[M_{G}\right]^{f l}\right)$, we have

(i) $H^{1}\left(G,\left[M_{G}\right]^{f l}\right) \simeq(\mathbb{Z} / 2 \mathbb{Z})^{\oplus 2}$ if and only if

(i-1) the CARAT ID of $G$ is one of the 6 triples $(5,31,26),(5,31,27),(5,664,2),(5,669,2),(5,670,2),(5,773,4)$ where $M_{G}$ is an indecomposable $G$-lattice of rank 5 and $G$ is isomorphic to $\left(C_{2}\right)^{3},\left(C_{2}\right)^{3}, C_{2} \times Q_{8},\left(C_{4} \times C_{2}\right) \rtimes C_{2}$, $\left(C_{4} \times C_{2}\right) \rtimes C_{2}, Q_{8}$ respectively; or

(i-2) the CARAT ID of $G$ is one of the 5 triples $(5,664,1),(5,773,3),(5,774,3),(5,691,1),(5,730,1)$ where $M_{G}$ is a decomposable $G$-lattice of rank $5=4+1$ and $G$ is isomorphic to $C_{2} \times Q_{8}, Q_{8}, Q_{8}, \mathrm{SL}_{2}\left(\mathbb{F}_{3}\right), C_{2} \times \mathrm{SL}_{2}\left(\mathbb{F}_{3}\right)$ respectively.

(ii) $H^{1}\left(G,\left[M_{G}\right]^{f l}\right) \simeq \mathbb{Z} / 2 \mathbb{Z}$ if and only if

(ii-1) the CARAT ID of $G$ is one of the 141 triples as in Example 4.5 where $M_{G}$ is an indecomposable G-lattice of rank 5 ;

(ii-2) the CARAT ID of $G$ is one of the 73 triples as in Example 4.5 where $M_{G}$ is a decomposable G-lattice of rank $5=4+1$;

(ii-3) the CARAT ID of $G$ is one of the 36 triples as in Example 4.5 where $M_{G}$ is a decomposable G-lattice of rank $5=3+2$; or

(ii-4) the CARAT ID of $G$ is one of the 13 triples as in Example 4.5 where $M_{G}$ is a decomposable G-lattice of $\operatorname{rank} 5=3+1+1$.

Proof. The method is the same as in the proof of Theorem 4.1. By [HY17, Theorem 1.12], among the 6079 cases of 5 -dimensional algebraic $k$-tori, there exist 3003 cases of algebraic $k$-tori which are not retract $k$-rational. The CARAT IDs of such 3003 groups $G \leq \mathrm{GL}_{5}(\mathbb{Z})$ with $\left[M_{G}\right]^{f l}$ is not invertible, are given in $[$ HY17, Tables 12, 13, 14, 15]. They are also given in [HY17, Example 4.12 and Example 11.1] as the lists N5, N41, N32, N311 when $M_{G}$ is indecomposable (resp. decomposable with rank $5=4+1,5=3+1,5=3+1+1$ ) and available from

https://www.math.kyoto-u.ac.jp/ ${ }^{\sim}$ yamasaki/Algorithm/MultInvField/NonInv5.dat.

Then we apply the functions FlabbyResolutionLowRank $(G)$.actionF in [HHY20, Algorithm 4.1] and H1 to get $H^{1}\left(G,\left[M_{G}\right]^{f l}\right)$ (see Example 4.5 and also the proof of Theorem 4.1). 
Example 4.3 (Classification of $H^{1}\left(G,\left[M_{G}\right]^{f l}\right) \neq 0$ for $G \leq \mathrm{GL}_{3}(\mathbb{Z})$ ).

gap> Read("FlabbyResolutionFromBase.gap") ;

gap> Read("NonInv.dat");

\# N3 is the list of GAP IDs (Crystcat IDs) of indecomposable lattice of rank 3

\# whose flabby class [M_G]`fl is not invertible [HY17, Example 4.12]

gap> N3;

$[[3,3,1,3],[3,3,3,3],[3,3,3,4],[3,4,3,2],[3,4,4,2]$,

$[3,4,6,3],[3,4,7,2],[3,7,1,2],[3,7,2,2],[3,7,2,3]$,

$[3,7,3,2],[3,7,3,3],[3,7,4,2],[3,7,5,2],[3,7,5,3]]$

gap> Length(N3); \# there exist 15 not retract rational tori in dim=3 [HY17, Table 1]

15

gap> N3g:=List (N3, $\mathrm{x}->$ MatGroupZClass (x [1] , $\mathrm{x}[2], \mathrm{x}[3], \mathrm{x}[4]))$; ;

gap> List (N3g, StructureDescription);

[ "C2 x C2", "C2 x C2 x C2", "C2 x C2 x C2", "C4 x C2", "D8", "D8", "C2 x D8",

"A4", "C2 x A4", "C2 x A4", "S4", "S4", "S4", "C2 x S4", "C2 x S4" ]

gap> N3gF:=List (N3g, $x->$ FlabbyResolutionLowRank $(x)$.actionF); ;

gap> N3H1F:=List (N3gF, $x->$ Filtered $(\mathrm{H} 1(\mathrm{x}), \mathrm{y}->\mathrm{y}>1)) ; \# \mathrm{H} 1(\mathrm{~F})$

$[[2],[],[],[],[],[],[],[2],[],[],[],[],[],[],[]]$

gap> N3H1FC2:=Filtered $([1 \ldots$ Length $(\mathrm{N} 3 g \mathrm{~F})], \mathrm{x}->\mathrm{N} 3 \mathrm{H} 1 \mathrm{~F}[\mathrm{x}]=[2])$;

$[1,8]$

gap> List $(\mathrm{N} 3 \mathrm{H} 1 \mathrm{FC} 2, \mathrm{x}->\mathrm{N} 3[\mathrm{x}]) ; \#$ GAP ID's of $\mathrm{F}$ with $\mathrm{H} 1(\mathrm{~F})=\mathrm{C} 2$

$[[3,3,1,3],[3,7,1,2]]$

gap> List (N3H1FC2, $\mathrm{x}->$ StructureDescription $(\mathrm{N} 3 \mathrm{~g}[\mathrm{x}]))$;

[ "C2 x $\mathrm{C} 2$ ", "A4" ]

Example 4.4 (Classification of $H^{1}\left(G,\left[M_{G}\right]^{f l}\right) \neq 0$ for $G \leq \mathrm{GL}_{4}(\mathbb{Z})$ ).

gap> Read("FlabbyResolutionFromBase.gap");

gap> Read("NonInv.dat");

\# N4 is the list of GAP IDs (Crystcat IDs) of indecomposable lattice of rank 4

\# whose flabby class [M_G]^fl is not invertible [HY17, Example 4.12]

gap> N4;

$[[4,5,1,12],[4,5,2,5],[4,5,2,8],[4,5,2,9],[4,6,1,6]$,

$[4,6,1,11],[4,6,2,6],[4,6,2,10],[4,6,2,12],[4,6,3,4]$,

$[4,6,3,7],[4,6,3,8],[4,12,2,5],[4,12,2,6],[4,12,3,11]$,

$[4,12,4,10],[4,12,4,11],[4,12,4,12],[4,12,5,8],[4,12,5,9]$,

$[4,12,5,10],[4,12,5,11],[4,13,1,5],[4,13,2,5],[4,13,3,5]$,

$[4,13,4,5],[4,13,5,4],[4,13,5,5],[4,13,6,5],[4,13,7,9]$,

$[4,13,7,10],[4,13,7,11],[4,13,8,5],[4,13,8,6],[4,13,9,4]$,

$[4,13,9,5],[4,13,10,4],[4,13,10,5],[4,18,1,3],[4,18,2,4]$,

$[4,18,2,5],[4,18,3,5],[4,18,3,6],[4,18,3,7],[4,18,4,4]$,

$[4,18,4,5],[4,18,5,5],[4,18,5,6],[4,18,5,7],[4,19,1,2]$,

$[4,19,2,2],[4,19,3,2],[4,19,4,3],[4,19,4,4],[4,19,5,2]$,

$[4,19,6,2],[4,22,1,1],[4,22,2,1],[4,22,3,1],[4,22,4,1]$,

$[4,22,5,1],[4,22,5,2],[4,22,6,1],[4,22,7,1],[4,22,8,1]$,

$[4,22,9,1],[4,22,10,1],[4,22,11,1],[4,24,2,4],[4,24,2,6]$,

$[4,24,4,4],[4,24,5,4],[4,24,5,6],[4,25,1,3],[4,25,2,3]$,

$[4,25,2,5],[4,25,3,3],[4,25,4,3],[4,25,5,3],[4,25,5,5]$,

$[4,25,6,3],[4,25,6,5],[4,25,7,3],[4,25,8,3],[4,25,9,3]$,

$[4,25,9,5],[4,25,10,3],[4,25,10,5],[4,25,11,3],[4,25,11,5]$,

$[4,29,1,1],[4,29,1,2],[4,29,2,1],[4,29,3,1],[4,29,3,2]$,

$[4,29,3,3],[4,29,4,1],[4,29,4,2],[4,29,5,1],[4,29,6,1]$,

$[4,29,7,1],[4,29,7,2],[4,29,8,1],[4,29,8,2],[4,29,9,1]$,

$[4,32,1,2],[4,32,2,2],[4,32,3,2],[4,32,4,2],[4,32,5,2]$,

$[4,32,5,3],[4,32,6,2],[4,32,7,2],[4,32,8,2],[4,32,9,4]$,

$[4,32,9,5],[4,32,10,2],[4,32,11,2],[4,32,11,3],[4,32,12,2]$,

$[4,32,13,3],[4,32,13,4],[4,32,14,3],[4,32,14,4],[4,32,15,2]$,

$[4,32,16,2],[4,32,16,3],[4,32,17,2],[4,32,18,2],[4,32,18,3]$,

$[4,32,19,2],[4,32,19,3],[4,32,20,2],[4,32,20,3],[4,32,21,2]$,

$[4,32,21,3],[4,33,1,1],[4,33,3,1],[4,33,4,1],[4,33,5,1]$,

$[4,33,6,1],[4,33,7,1],[4,33,8,1],[4,33,9,1],[4,33,10,1]$,

$[4,33,11,1],[4,33,12,1],[4,33,13,1],[4,33,14,1],[4,33,14,2]$,

$[4,33,15,1],[4,33,16,1]]$

gap> Length(N4); \# there exist 152 not retract rational tori in dim=4 [HY17, Table 4] 
152

gap> N4g:=List (N4, $x->$ MatGroupZClass ( $x[1], x[2], x[3], x[4]))$; ;

gap> N4gF:=List (N4g, $\mathrm{x}->\mathrm{FlabbyResolutionLowRank}(\mathrm{x})$.actionF); ;

gap> N4H1F : =List (N4gF, $x->$ Filtered $(H 1(x), y->y>1))$; ;

gap> Collected $(\mathrm{N} 4 \mathrm{H} 1 \mathrm{~F})$;

$[[[], 135],[[2], 15],[[2,2], 2]]$

gap> N4H1FC2xC2:=Filtered ( $[1 \ldots$ Length $(\mathrm{N} 4 \mathrm{H} 1 \mathrm{~F})], \mathrm{x}->\mathrm{N} 4 \mathrm{H} 1 \mathrm{~F}[\mathrm{x}]=[2,2])$;

[ 106,138$]$

gap> List $(\mathrm{N} 4 \mathrm{H} 1 \mathrm{FC} 2 \mathrm{xC} 2, \mathrm{x}->\mathrm{N} 4[\mathrm{x}])$; \# GAP ID's of $\mathrm{F}$ with $\mathrm{H} 1(\mathrm{~F})=\mathrm{C} 2 \mathrm{xC} 2$

$[[4,32,1,2],[4,33,3,1]]$

gap> List (N4H1FC2xC2, $\mathrm{x}->$ StructureDescription (N4g $[\mathrm{x}])$ );

[ "Q8", "SL $(2,3)$ " ]

gap> N4H1FC2:=Filtered ( $[1 \ldots$ Length $(\mathrm{N} 4 \mathrm{H} 1 \mathrm{~F})], \mathrm{x}->\mathrm{N} 4 \mathrm{H} 1 \mathrm{~F}[\mathrm{x}]=[2])$;

$[1,3,8,14,18,21,39,45,107,108,109,112,140,141,144]$

gap> List $(\mathrm{N} 4 \mathrm{H} 1 \mathrm{FC} 2, \mathrm{x}->\mathrm{N} 4[\mathrm{x}])$; \# GAP ID's of $\mathrm{F}$ with $\mathrm{H} 1(\mathrm{~F})=\mathrm{C} 2$

$[[4,5,1,12],[4,5,2,8],[4,6,2,10],[4,12,2,6],[4,12,4,12]$,

$[4,12,5,10],[4,18,1,3],[4,18,4,4],[4,32,2,2],[4,32,3,2]$,

$[4,32,4,2],[4,32,6,2],[4,33,5,1],[4,33,6,1],[4,33,9,1]]$ gap> List (N4H1FC2, $\mathrm{x}->$ StructureDescription (N4g $[\mathrm{x}])$ );

[ "C2 x C2", "C2 x C2 x C2", "C2 x C2 x C2", "C4 x C2", "D8", "C2 x D8", "C4 x C2",

"C2 x D8", "C8 : C2", "QD16", "(C4 x C2) : C2", "C8 : (C2 x C2)",

"((C4 x C2) : C2) : C3", "GL $(2,3) "$, "GL $(2,3): C 2 "]$

\# N31 is the list of GAP IDs (Crystcat IDs) of decomposable lattice of rank 4=3+1

\# whose flabby class [M_G] ${ }^{` f l}$ is not invertible [HY17, Example 4.12]

gap> N31;

$[[4,4,3,6],[4,4,4,4],[4,4,4,6],[4,5,1,9],[4,5,2,4]$,

$[4,5,2,7],[4,6,1,4],[4,6,1,8],[4,6,2,4],[4,6,2,8]$,

$[4,6,2,9],[4,6,3,3],[4,6,3,6],[4,7,3,2],[4,7,4,3]$,

$[4,7,5,2],[4,7,7,2],[4,12,2,4],[4,12,3,7],[4,12,4,6]$,

$[4,12,4,8],[4,12,4,9],[4,12,5,6],[4,12,5,7],[4,13,1,3]$,

$[4,13,2,4],[4,13,3,4],[4,13,4,3],[4,13,5,3],[4,13,6,3]$,

$[4,13,7,6],[4,13,7,7],[4,13,7,8],[4,13,8,3],[4,13,8,4]$,

$[4,13,9,3],[4,13,10,3],[4,24,1,5],[4,24,2,3],[4,24,2,5]$,

$[4,24,3,5],[4,24,4,3],[4,24,4,5],[4,24,5,3],[4,24,5,5]$,

$[4,25,1,2],[4,25,1,4],[4,25,2,4],[4,25,3,2],[4,25,3,4]$,

$[4,25,4,4],[4,25,5,2],[4,25,5,4],[4,25,6,2],[4,25,6,4]$,

$[4,25,7,2],[4,25,7,4],[4,25,8,2],[4,25,8,4],[4,25,9,4]$,

$[4,25,10,2],[4,25,10,4],[4,25,11,2],[4,25,11,4]]$

gap> Length(N31); \# there exist 64 not retract rational tori in dim=4=3+1 [HY17, Table 3]

64

gap> N31g:=List (N31, $x->$ MatGroupZClass ( $[1], x[2], x[3], x[4])) ;$;

gap> N31gF:=List (N31g, $\mathrm{x}->\mathrm{FlabbyResolutionLowRank}(\mathrm{x})$.actionF); ;

gap> N31H1F : $=$ List $(N 31 g F, x->F i l t e r e d(H 1(x), y->y>1)) ;$;

gap> Collected (N31H1F);

[ [ [ ], 59$],[[2], 5]]$

gap> N31H1FC2: =Filtered $([1 \ldots$ Length $(N 31 H 1 F)], x->N 31 H 1 F[x]=[2])$;

$[1,4,11,38,48]$

gap> List $(\mathrm{N} 31 \mathrm{H} 1 \mathrm{FC} 2, \mathrm{x}->\mathrm{N} 31[\mathrm{x}])$; \# GAP ID's of $\mathrm{F}$ with $\mathrm{H} 1(\mathrm{~F})=\mathrm{C} 2$

$[[4,4,3,6],[4,5,1,9],[4,6,2,9],[4,24,1,5],[4,25,2,4]]$

gap> List (N31H1FC2, $\mathrm{x}->$ StructureDescription (N31g $[\mathrm{x}])$ );

[ "C2 x C2", "C2 x C2", "C2 x C2 x C2", "A4", "C2 x A4"]

Example 4.5 (Classification of $H^{1}\left(G,\left[M_{G}\right]^{f l}\right) \neq 0$ for $\left.G \leq \mathrm{GL}_{5}(\mathbb{Z})\right)$.

gap> Read("FlabbyResolutionFromBase.gap") ;

gap> Read("caratnumber.gap");

gap> Read("NonInv5.dat");

\# N5 is the list of CARAT IDs of indecomposable lattice of rank 5

\# whose flabby class [M_G] $f 1$ is not invertible [HY17, Example 4.12]

gap> N5g:=List (N5, x->CaratMatGroupZClass $(x[1], x[2], x[3])) ;$; 
gap> Length(N5g); \# there exist 1141 not retract rational tori in dim=5 [HY17, Table 15] 1141

gap> N5gF:=List (N5g, $\mathrm{x}->\mathrm{FlabbyResolutionLowRank(x)}$.actionF); ;

gap> N5H1F: $=$ List (N5gF, $\mathrm{x}->$ Filtered $(\mathrm{H} 1(\mathrm{x}), \mathrm{y}->\mathrm{y}>1)) ;$

gap> Collected (N5H1F);

$[[[], 994],[[2], 141],[[2,2], 6]]$

gap> N5H1FC2xC2:=Filtered ( $[1 .$. Length $(\mathrm{N} 5 \mathrm{H} 1 \mathrm{~F})], \mathrm{x}->\mathrm{N} 5 \mathrm{H} 1 \mathrm{~F}[\mathrm{x}]=[2,2])$;

$[69,70,906,913,915,1064]$

gap> List $(\mathrm{N} 5 \mathrm{H} 1 \mathrm{FC} 2 \mathrm{xC2}, \mathrm{x}->\mathrm{N} 5[\mathrm{x}])$; \# CARAT ID's of $\mathrm{F}$ with $\mathrm{H} 1(\mathrm{~F})=\mathrm{C} 2 \mathrm{xC} 2$

$[[5,31,26],[5,31,27],[5,664,2],[5,669,2],[5,670,2],[5,773,4]]$ gap> List (N5H1FC2xC2, x->StructureDescription(N5g[x]));

[ "C2 2 C2 $\times$ C2", "C2 x C2 x C2", "C2 x Q8", "(C4 x C2) : C2", "(C4 x C2) : C2", "Q8" ]

gap> N5H1FC2:=Filtered ( $[1 \ldots$ Length $(\mathrm{N} 5 \mathrm{H} 1 \mathrm{~F})], \mathrm{x}->\mathrm{N} 5 \mathrm{H} 1 \mathrm{~F}[\mathrm{x}]=[2])$;

$[3,4,5,6,8,11,19,20,27,36,37,42,43,61,63,67,71,72,74,78,79,86,88,89$, $96,99,100,103,115,116,128,129,130,131,142,143,158,159,160,173,174,178$, $179,185,186,187,188,191,193,199,200,221,222,238,242,243,253,254,288,292$, $293,316,317,318,324,327,331,333,334,337,339,348,358,362,375,376,378,389$, $401,403,404,406,407,410,414,419,423,425,440,470,480,495,511,523,540,573$, $588,590,591,592,593,595,596,597,606,680,715,723,762,852,853,854,855,908$, $909,912,916,918,921,922,948,957,961,964,970,971,973,974,976,980,982,984$, $1037,1060,1065,1114,1115,1116,1117,1129,1130]$

gap> List (N5H1FC2, $\mathrm{x}->\mathrm{N} 5[\mathrm{x}])$; \# CARAT ID's of $\mathrm{F}$ with $\mathrm{H} 1(\mathrm{~F})=\mathrm{C} 2$

$[[5,18,23],[5,19,17],[5,20,14],[5,20,17],[5,21,17],[5,24,23]$, $[5,25,27],[5,25,28],[5,26,21],[5,26,40],[5,26,41],[5,27,14]$, $[5,27,15],[5,30,24],[5,30,28],[5,31,18],[5,31,31],[5,31,32]$, $[5,31,36],[5,31,44],[5,31,45],[5,32,36],[5,32,44],[5,32,51]$, $[5,39,5],[5,71,19],[5,71,22],[5,71,25],[5,72,34],[5,72,36]$, $[5,73,32],[5,73,34],[5,73,36],[5,73,37],[5,75,34],[5,75,36]$, $[5,76,49],[5,76,50],[5,76,51],[5,78,12],[5,78,15],[5,78,28]$, $[5,78,31],[5,79,12],[5,79,15],[5,79,17],[5,79,18],[5,79,31]$, $[5,79,36],[5,80,12],[5,80,15],[5,83,15],[5,83,17],[5,86,9]$, $[5,87,9],[5,87,11],[5,88,34],[5,88,36],[5,93,9],[5,94,9]$, $[5,94,11],[5,99,23],[5,99,24],[5,99,25],[5,100,12],[5,100,23]$, $[5,100,28],[5,101,17],[5,101,18],[5,102,9],[5,102,17]$,

$[5,105,5],[5,109,5],[5,109,14],[5,112,5],[5,112,7],[5,113,4]$, $[5,116,20],[5,118,18],[5,119,4],[5,119,5],[5,119,10]$, $[5,119,12],[5,120,5],[5,120,14],[5,121,13],[5,122,9]$, $[5,122,15],[5,127,11],[5,134,9],[5,136,18],[5,140,23]$, $[5,142,14],[5,143,23],[5,148,5],[5,154,15],[5,160,4]$, $[5,160,7],[5,161,5],[5,161,7],[5,162,5],[5,224,9],[5,227,11]$, $[5,232,14],[5,242,9],[5,526,11],[5,534,11],[5,536,13]$,

$[5,546,11],[5,580,2],[5,604,2],[5,604,4],[5,605,2],[5,665,4]$, $[5,666,4],[5,668,2],[5,670,3],[5,671,2],[5,672,2],[5,673,2]$, $[5,704,3],[5,706,8],[5,708,2],[5,709,3],[5,713,2],[5,714,2]$, $[5,715,2],[5,716,2],[5,717,2],[5,719,2],[5,720,2],[5,721,2]$, $[5,763,3],[5,770,2],[5,774,4],[5,948,1],[5,948,2],[5,948,3]$, $[5,948,4],[5,952,1],[5,952,3]]$

gap> List (N5H1FC2, $\mathrm{x}->$ StructureDescription (N5g $[\mathrm{x}])$ ) ;

[ "C2 x C2", "C2 x C2", "C2 x C2 x C2", "C2 x C2 x C2", "C2 x C2 x C2", "C2 x C2 x C2", "C2 x C2 x C2 x C2", "C2 x C2 x C2 x C2", "C2 x C2 x C2 x C2", "C2 x C2 x C2 x C2", "C2 x C2 x C2 x C2", "C2 x C2 x C2 x C2", "C2 x C2 x C2 x C2", "C2 x C2 x C2", "C2 x C2 x C2", "C2 x C2 x C2", "C2 x C2 x C2", "C2 x C2 x C2", "C2 x C2 x C2", "C2 x C2 x C2", "C2 x C2 x C2", "C2 x C2 x C2", "C2 x C2 x C2", "C2 x C2 x C2", "D8", "C2 x D8", "C2 x D8", "C2 x D8", "C2 x D8", "C2 x D8", "C2 x D8", "C2 x D8", "C2 x D8", "C2 x D8", "C2 x D8", "C2 x D8", "C2 x D8", "C2 x D8", "C2 x D8", "C2 x D8", "C2 x D8", "C2 x D8", "C2 x D8", "C2 x D8", "C2 x D8", "C2 x D8", "C2 x D8", "C2 x D8", "C2 x D8", "C2 x D8", "C2 x D8", "C4 x C2 x C2", "C4 x C2 x C2", "C4 x C2 x C2", "C4 x C2 x C2", "C4 x C2 x C2", "C2 x C2 x D8", "C2 x C2 x D8", "C2 x C2 x D8", "C2 x C2 x D8", "C2 x C2 x D8", "D8", "D8", "D8", "D8", "D8", "D8", "C4 x C2", "C4 x C2", "C4 x C2", "C4 x C2", "C4 : C4", "(C4 x C2) : C2", "(C4 x C2) : C2", "C4 x C2 x C2", "C4 x C2 x C2", "C4 x C4", "(C4 x C2) : C2", "(C4 x C2) : C2", "C2 x D8", "C2 x D8", "C2 x D8", "C2 x D8", 
"C2 x D8", "C2 x D8", "C2 x D8", "C2 x D8", "C2 x D8", "C4 x D8", "(C4 x C4) : C2", "( 2 x $\mathrm{C} 2 \times \mathrm{C} 2 \times \mathrm{C} 2): \mathrm{C} 2 ", "(\mathrm{C} 4 \times \mathrm{C} 2 \times \mathrm{C} 2): \mathrm{C} 2 ", "(\mathrm{C} 2 \times \mathrm{C} 2 \times \mathrm{C} 2 \times \mathrm{C} 2): \mathrm{C} 2 "$, "(C4 x C2 x C2) : C2", "C2 x C2 x D8", "D8 x D8", "C4 x C2", "C4 x C2", "C4 x C2", "C4 x C2", "C4 x C2", "C6 x C2", "D12", "D12", "C2 x C2 x S3", "C2 x C2 x A4", "C2 x S4", "C2 x S4", "C2 x C2 x S4", "A4", "C2 x A4", "C2 x A4", "C2 x A4", "C8 : C2", "C8 : C2", "(C4 x C2) : C2", "(C4 x C2) : C2", "(C4 x C2) : C2", "QD16", "QD16", "(C2 x C2 x C2) : C4", "( 2 x $\mathrm{C} 2 \times \mathrm{C} 2): \mathrm{C} 4 ", "(\mathrm{C} 2 \times \mathrm{C} 2 \times \mathrm{C} 2):(\mathrm{C} 2 \times \mathrm{C} 2) ", "(\mathrm{C} 2 \times \mathrm{C} 2 \times \mathrm{C} 2):(\mathrm{C} 2 \times \mathrm{C} 2) "$, "C8 : ( 2 x $\mathrm{C} 2)$ ", "C8 : ( 2 x $\mathrm{C} 2)$ ", "C8 : (C2 x C2)", "C8 : (C2 x C2)", "C8 : (C2 x C2)", "C2 x ((C4 x C2) : C2)", "C2 x (C8 : C2)", "C2 x QD16", "((C2 x C2 x C2) : (C2 x C2)) : C2", "C2 x (C8 : (C2 x C2))", "Q8", "C2 x A5", "C2 x A5", "C2 x A5", "C2 x A5", "A5", "A5" ]

\# N41 is the list of CARAT IDs of decomposable lattice of rank $5=4+1$ \# whose flabby class [M_G]^fl is not invertible [HY17, Example 4.12] gap> N41g:=List (N41, $\mathrm{x}->$ CaratMatGroupZClass (x[1] , $\mathrm{x}[2], \mathrm{x}[3])$ ); ;

gap> Length(N41g); \# there exist 768 not retract rational tori in dim=5=4+1 [HY17, Table 14] 768

gap> N41gF:=List (N41g, $\mathrm{x}->\mathrm{FlabbyResolutionLowRank}(\mathrm{x})$.actionF); ;

gap> N41H1F:=List (N41gF, $\mathrm{x}->$ Filtered $(\mathrm{H} 1(\mathrm{x}), \mathrm{y}->\mathrm{y}>1))$; ;

gap> Collected (N41H1F);

$[[[], 690],[[2], 73],[[2,2], 5]]$

gap> N41H1FC2xC2:=Filtered ( $[1 \ldots$ Length $(\mathrm{N} 41 \mathrm{H} 1 \mathrm{~F})], \mathrm{x}->\mathrm{N} 41 \mathrm{H} 1 \mathrm{~F}[\mathrm{x}]=[2,2])$;

[ 589, 590, 591, 720,721]

gap> List (N41H1FC2xC2, $\mathrm{x}->\mathrm{N} 41[\mathrm{x}])$;

$[[5,664,1],[5,773,3],[5,774,3],[5,691,1],[5,730,1]]$

gap> List (N41H1FC2 $\mathrm{xC2}, \mathrm{x}->$ StructureDescription (N41g $[\mathrm{x}])$ );

[ "C2 x Q8", "Q8", "Q8", "SL $(2,3) ", ~ " C 2 \times \operatorname{SL}(2,3) "]$

gap> N41H1FC2:=Filtered ( $[1 \ldots$ Length $(\mathrm{N} 41 \mathrm{H} 1 \mathrm{~F})], \mathrm{x}->\mathrm{N} 41 \mathrm{H} 1 \mathrm{~F}[\mathrm{x}]=[2])$;

$[1,2,3,4,9,10,11,12,13,14,36,37,38,39,40,41,42,74,75,76,77,93,94,95$, $96,97,112,113,114,115,116,117,118,254,255,256,257,281,282,283,284,285$, $592,593,594,595,596,597,598,599,600,601,602,603,604,605,610,611,612,613$, $614,615,616,727,728,729,730,731,732,738,739,740,741]$

gap> List $(\mathrm{N} 41 \mathrm{H} 1 \mathrm{FC} 2, \mathrm{x}->\mathrm{N} 41[\mathrm{x}])$; \# CARAT ID's of $\mathrm{F}$ with $\mathrm{H} 1(\mathrm{~F})=\mathrm{C} 2$

$[[5,18,18],[5,18,21],[5,19,10],[5,32,23],[5,20,10],[5,20,13]$, $[5,25,14],[5,30,14],[5,31,16],[5,31,29],[5,21,10],[5,24,18]$, $[5,24,21],[5,26,19],[5,31,22],[5,31,25],[5,32,30],[5,66,5]$, $[5,83,7],[5,101,4],[5,102,4],[5,63,12],[5,65,12],[5,76,31]$, $[5,99,5],[5,100,5],[5,48,12],[5,71,8],[5,72,26],[5,75,26]$, $[5,78,26],[5,79,26],[5,88,26],[5,112,3],[5,160,3],[5,161,3]$, $[5,162,3],[5,119,3],[5,120,11],[5,121,11],[5,122,14]$,

$[5,148,3],[5,665,3],[5,666,3],[5,667,3],[5,720,1],[5,672,1]$, $[5,673,1],[5,674,1],[5,675,1],[5,721,1],[5,668,1],[5,669,1]$, $[5,670,1],[5,671,1],[5,719,1],[5,713,1],[5,714,1],[5,715,1]$, $[5,716,1],[5,717,1],[5,718,1],[5,770,1],[5,731,1],[5,732,1]$, $[5,775,1],[5,733,1],[5,734,1],[5,776,1],[5,682,1],[5,780,1]$, $[5,781,1],[5,783,1]]$

gap> List (N41H1FC2, $\mathrm{x}->$ StructureDescription (N41g $[\mathrm{x}])$ );

[ "C2 x C2", "C2 x C2", "C2 x C2", "C2 x C2 x C2", "C2 x C2 x C2", "C2 x C2 x C2",

"C2 x C2 x C2 x C2", "C2 x C2 x C2", "C2 x C2 x C2", "C2 x C2 x C2", "C2 x C2 x C2",

"C2 x C2 x C2", "C2 x C2 x C2", "C2 x C2 x C2 x C2", "C2 x C2 x C2", "C2 x C2 x C2",

"C2 x C2 x C2", "C4 x C2", "C4 x C2 x C2", "C4 4 C2", "C4 x C2", "D8", "D8", "C2 x D8", "D8", "D8", "C2 x D8", "C2 x D8", "C2 x D8", "C2 x D8", "C2 x D8", "C2 x D8", "C2 x C2 x D8", "C4 x C2 x C2", "C4 x C2", "C4 x C2", "C4 x C2", "C2 x D8", "C2 x D8", "C2 x D8", "C2 x D8", "C2 x C2 x D8", "C8 : C2", "C8 : C2", "C8 : C2", "C2 x (C8 : C2)", "QD16", "QD16", "QD16", "QD16", "C2 x QD16", "(C4 x C2) : C2", "(C4 x C2) : C2", "(C4 x C2) : C2", "(C4 x C2) : C2", "C2 x ((C4 x C2) : C2)", "C8 : (C2 x C2)",

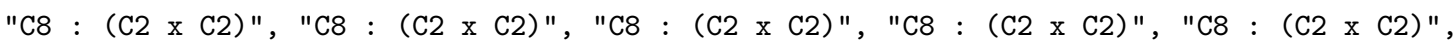
"C2 x (C8 : (C2 x C2))", "((C4 x C2) : C2) : C3", "((C4 x C2) : C2) : C3",

"C2 x (((C4 x C2) : C2) : C3)", "GL $(2,3) "$, "GL $(2,3) "$, "C2 x GL $(2,3)$ ", "C2 x $(\mathrm{GL}(2,3): \mathrm{C} 2) "$, $"(((\mathrm{C} 4 \times \mathrm{C} 2): \mathrm{C} 2): \mathrm{C} 3): \mathrm{C} 2 ", "(((\mathrm{C} 4 \times \mathrm{C} 2): \mathrm{C} 2): \mathrm{C} 3): \mathrm{C} 2 "$,

"(((C4 x C2) : C2 $): \mathrm{C} 3): \mathrm{C} 2 "]$ 
\# N32 is the list of CARAT IDs of decomposable lattice of rank $5=3+2$

\# whose flabby class [M_G] $f 1$ is not invertible [HY17, Example 4.12]

gap> N32g:=List (N32, $\mathrm{x}->$ CaratMatGroupZClass $(\mathrm{x}[1], \mathrm{x}[2], \mathrm{x}[3]))$; ;

gap> Length(N32g); \# there exist 849 not retract rational tori in dim=5=3+2 [HY17, Table 13]

849

gap> N32gF:=List (N32g, $\mathrm{x}->\mathrm{FlabbyResolutionLowRank(x)}$.actionF); ;

gap> N32H1F : $=$ List $(N 32 \mathrm{gF}, \mathrm{x}->$ Filtered $(\mathrm{H} 1(\mathrm{x}), \mathrm{y}->\mathrm{y}>1))$; ;

gap> Collected (N32H1F);

$[[[], 813],[[2], 36]]]$

gap> N32H1FC2:=Filtered ( $[1 \ldots$ Length $(\mathrm{N} 32 \mathrm{H} 1 \mathrm{~F})], \mathrm{x}->\mathrm{N} 32 \mathrm{H} 1 \mathrm{~F}[\mathrm{x}]=[2])$;

$[1,2,3,4,5,6,7,8,9,10,11,12,13,14,15,16,17,18,19,20,21$,

$22,23,24,25,578,579,580,581,582,583,584,585,586,587,588$ ]

gap> List $(\mathrm{N} 32 \mathrm{H} 1 \mathrm{FC} 2, \mathrm{x}->\mathrm{N} 32[\mathrm{x}])$; \# CARAT ID's of $\mathrm{F}$ with $\mathrm{H} 1(\mathrm{~F})=\mathrm{C} 2$

$[[5,14,8],[5,18,19],[5,24,19],[5,26,20],[5,31,17]$,

$[5,32,17],[5,78,8],[5,78,27],[5,80,8],[5,86,5]$,

$[5,93,5],[5,100,11],[5,102,8],[5,224,4],[5,227,5]$,

$[5,228,3],[5,232,4],[5,232,9],[5,237,3],[5,242,4]$,

$[5,242,14],[5,247,3],[5,247,7],[5,253,4]$,

$[5,259,3],[5,520,17],[5,525,2],[5,560,3]$,

$[5,566,3],[5,580,1],[5,590,1],[5,605,1],[5,620,1]$,

$[5,629,1],[5,634,1],[5,634,3]]$

gap> List (N32H1FC2, $\mathrm{x}->$ StructureDescription (N32g $[\mathrm{x}])$ ) ;

[ "C2 x C2", "C2 x C2", "C2 x C2 x C2", "C2 x C2 x C2 x C2", "C2 x C2 x C2",

"C2 x C2 x C2", "C2 x D8", "C2 x D8", "C2 x D8", "C4 x C2 x C2",

"C2 x C2 x D8", "D8", "C4 x C2", "C6 x C2", "D12", "C6 x C2", "D12",

"D12", "C6 x C2 x C2", "C2 x C2 x S3", "C2 x C2 x S3", "C2 x C2 x S3",

"C2 x C2 x S3", "C2 x C2 x S3", "C2 x C2 x C2 x S3", "C2 x A4",

"C2 x C2 x A4", "C4 x A4", "A4 x D8", "A4", "C2 x A4 x S3", "C2 x A4",

"C3 x A4", "C6 x A4", "A4 x S3", "A4 x S3" ]

\# N311 is the list of CARAT IDs of decomposable lattice of rank $5=3+1+1$

\# whose flabby class [M_G]^fl is not invertible [HY17, Example 4.12]

gap> N311g:=List (N311, $\mathrm{x}->$ CaratMatGroupZClass (x [1], $\mathrm{x}[2], \mathrm{x}[3])$ ); ;

gap> Length(N311g); \# there exist 245 not retract rational tori in dim=5=3+1+1 [HY17, Table 12]

245

gap> N311gF:=List (N311g, $\mathrm{x}->$ FlabbyResolutionLowRank $(\mathrm{x})$.actionF); ;

gap> N311H1F : =List (N311gF, $x->$ Filtered $(H 1(x), y->y>1)) ;$;

gap> Collected $(\mathrm{N} 311 \mathrm{H} 1 \mathrm{~F})$;

$[[[], 232],[[2], 13]]$

gap> N311H1FC2: $=$ Filtered $([1 \ldots$ Length $(\mathrm{N} 311 \mathrm{H} 1 \mathrm{~F})], \mathrm{x}->\mathrm{N} 311 \mathrm{H} 1 \mathrm{~F}[\mathrm{x}]=[2])$;

$[1,2,3,4,5,6,7,8,9,164,165,166,167]$

gap> List $(\mathrm{N} 311 \mathrm{H} 1 \mathrm{FC} 2, \mathrm{x}->\mathrm{N} 311[\mathrm{x}]) ;$ \# CARAT ID's of $\mathrm{F}$ with $\mathrm{H} 1(\mathrm{~F})=\mathrm{C} 2$

$[[5,11,4],[5,14,4],[5,18,7],[5,19,5],[5,21,5]$,

$[5,24,7],[5,26,3],[5,31,4],[5,32,10],[5,502,6]$,

$[5,505,1],[5,520,16],[5,525,1]]$

gap> List (N311H1FC2, $\mathrm{x}->$ StructureDescription(N311g $[\mathrm{x}]$ )) ;

[ "C2 x C2", "C2 x C2", "C2 x C2", "C2 x C2", "C2 x C2 x C2",

"C2 x C2 x C2", "C2 x C2 x C2 x C2", "C2 x C2 x C2", "C2 x C2 x C2",

"A4", "C2 x A4", "C2 x A4", "C2 x $\mathrm{C} 2 \times \mathrm{A} 4$ " ]

\section{Proof of Theorem 1.15 and Theorem 1.17}

Let $T=R_{K / k}^{(1)}\left(\mathbb{G}_{m}\right)$ be a norm one torus of $K / k$. We have the character module $\widehat{T}=J_{G / H}$ of $T$ and then $H^{1}(k, \operatorname{Pic} \bar{X}) \simeq H^{1}\left(G,\left[J_{G / H}\right]^{f l}\right.$ ) (see Section 2). We may assume that $H$ is the stabilizer of one of the letters in $G$, i.e. $L=k\left(\theta_{1}, \ldots, \theta_{n}\right)$ and $K=k\left(\theta_{i}\right)$ for some $1 \leq i \leq n$. In order to compute $H^{1}\left(G,\left[J_{G / H}\right]^{f l}\right)$, we apply the functions Norm1TorusJ $(n, m)$ and FlabbyResolutionLowRankFromGroup $(G, n T m)$.actionF in [HHY20, Algorithm 4.1]. Norm1Torus $(n, m)$ returns $J_{G / H}$ for $G=n T m \leq S_{n}$ and $H$ is the stabilizer of one of the letters in $G$ and FlabbyResolutionLowRankFromGroup $(G, n T m)$.actionF returns a suitable flabby class $F=\left[J_{G / H}\right]^{f l}$ with low rank by using the backtracking techniques for $G=n T m \leq S_{n}$.

Proof of Theorem 1.15 
For $G=n T m(2 \leq n \leq 11)$, the computation is described in Example 5.1 .

For $G=n T m(12 \leq n \leq 15)$, it needs much time and computer resources (memory) in computations. At present, we do not know the complete solutions for $n=12$.

For $13 \leq n \leq 15$, we wish to compute $H^{1}\left(G,\left[J_{G / H}\right]^{f l}\right)$ for each of the cases $G=13 T m(1 \leq m \leq 9), G=14 T m$ $(1 \leq m \leq 63), G=15 T m(1 \leq m \leq 104)$. This is achievable except for the each last two groups $G=13 T 8 \simeq A_{13}$, $13 T 9 \simeq S_{13}, 14 T 62 \simeq A_{14}, 14 T 63 \simeq S_{14}, 15 T 103 \simeq A_{15}, 15 T 104 \simeq S_{15}$ because of the computer resources reason (see Example 5.2). However, for the exceptional cases, we already know that $H^{1}\left(G,\left[J_{G / H}\right]^{f l}\right)=0$ by Theorem 1.10 and Theorem 1.11 .

The last assertion follows from Theorem 1.2 .

\section{Proof of Theorem 1.17}

For $G=11 T 6 \simeq M_{11} \leq S_{11}$ and $G=23 T 5 \simeq M_{23} \leq S_{23}$, it follows from Theorem 2.1 that $H^{1}\left(G,\left[J_{G / H}\right]^{f l}\right)=0$ (see also the paragraph after Theorem [3.6). For $G=24 T 24680 \simeq M_{24} \leq S_{24}$, we know that the Schur multiplier of $G$ vanishes: $M(G) \simeq H^{3}(G, \mathbb{Z})=0$ (see Mazet Maz82]). We know that the Mathieu groups are simple groups and a subgroup $H \leq M_{24}$ with $\left[M_{24}: H\right]=24$ is isomorphic to $M_{23}$ which is the stabilizer of one of the letters in $M_{24}$ (see e.g. [DM96, Exercises 6.8.8]). Hence it follows from $0=H^{a b} \simeq H^{2}(H, \mathbb{Z}) \simeq H^{2}(G, \mathbb{Z}[G / H]) \rightarrow$ $H^{2}\left(G, J_{G / H}\right) \stackrel{\delta}{\rightarrow} H^{3}(G, \mathbb{Z})=0$ that $H^{2}\left(G, J_{G / H}\right)=0$. Thus we have $H^{1}\left(G,\left[J_{G / H}\right]^{f l}\right) \simeq \amalg_{\omega}^{2}\left(G, J_{G / H}\right)=0$.

For $G=12 T 295 \simeq M_{12} \leq S_{12}$ and $G=22 T 38 \simeq M_{22} \leq S_{22}$, the computation is described in Example 5.3 .

Some related functions for Example 5.1. Example 5.2 and Example 5.3 are available from https://www . math.kyoto-u.ac.jp/ yamasaki/Algorithm/RatProbNorm1Tori/.

Example 5.1 (Computation of $H^{1}\left(G,\left[J_{G / H}\right]^{f l}\right.$ ) where $G=n T m(n \leq 11)$ ).

gap> Read ("FlabbyResolutionFromBase.gap") ;

gap> for $n$ in [2..11] do for $m$ in [1..NrTransitiveGroups(n)] do

$>$ F: $=$ FlabbyResolutionLowRankFromGroup(Norm1TorusJ (n,m), TransitiveGroup (n,m)) .actionF;

$>\operatorname{Print}([\mathrm{n}, \mathrm{m}], \operatorname{Length}(\mathrm{F} .1)$, Filtered (H1 (F), $\mathrm{x}->\mathrm{x}>1)]$, "\n"); od;Print ("\n" ); od;

$[[2,1], 1,[]]$

$[[3,1], 1,[]]$

$[[3,2], 4,[]]$

$[[4,1], 1,[]]$

[ $[4,2], 5,[2]]$

$[[4,3], 7,[]]$

[ [ 4,4$], 9,[2]]$

$[[4,5], 15,[]]$

$[[5,1], 1,[]]$

$[[5,2], 6,[]]$

$[[5,3], 16,[]]$

$[[5,4], 16,[]]$

$[[5,5], 16,[]]$

$[[6,1], 1,[]]$

$[[6,2], 7,[]]$

$[[6,3], 9,[]]$

$[[6,4], 10,[2]]$

$[[6,5], 21,[]]$

$[[6,6], 10,[]]$

$[[6,7], 19,[]]$

$[[6,8], 19,[]]$

$[[6,9], 27,[]]$

$[[6,10], 27,[]]$

$[[6,11], 19,[]]$

$[[6,12], 10,[2]]$

$[[6,13], 27,[]]$

$[[6,14], 31,[]]$

$[[6,15], 60,[]]$

$[[6,16], 60,[]]$

$[[7,1], 1,[]]$ 
$[[7,2], 8,[]]$

$[[7,3], 15,[]]$

$[[7,4], 36,[]]$

$[[7,5], 15,[]]$

$[[7,6], 36,[]]$

$[[7,7], 36,[]]$

$[[8,1], 1,[]]$

$[[8,2], 9,[2]]$

$[[8,3], 17,[2,2,2]]$

$[[8,4], 9,[2]]$

$[[8,5], 9,[]]$

$[[8,6], 11,[]]$

$[[8,7], 21,[]]$

$[[8,8], 11,[]]$

$[[8,9], 21,[2]]$

$[[8,10], 21,[]]$

[ $[8,11], 21,[2]]$

$[[8,12], 25,[]]$

$[[8,13], 19,[2]]$

$[[8,14], 13,[2]]$

$[[8,15], 43,[2]]$

$[[8,16], 29,[]]$

$[[8,17], 43,[]]$

$[[8,18], 91,[]]$

$[[8,19], 51,[2]]$

$[[8,20], 29,[]]$

[ $[8,21], 49,[2]]$

[ $[8,22], 49,[2]]$

$[[8,23], 31,[]]$

$[[8,24], 31,[]]$

$[[8,25], 49,[]]$

$[[8,26], 67,[]]$

$[[8,27], 29,[]]$

[ $[8,28], 83,[]]$

$[[8,29], 99,[]]$

$[[8,30], 67,[]]$

[ $[8,31], 49,[2]]$

$[[8,32], 61,[2]]$

$[[8,33], 99,[]]$

$[[8,34], 123,[]]$

$[[8,35], 99,[]]$

$[[8,36], 49,[]]$

$[[8,37], 49,[2]]$

$[[8,38], 61,[2]]$

$[[8,39], 211,[]]$

[ $[8,40], 115,[]]$

[ [ 8,41], 123, [ ] ]

[ $[8,42], 123,[]]$

[ $[8,43], 91,[]]$

[ $[8,44], 211,[]]$

$[[8,45], 123,[]]$

[ $[8,46], 123,[]]$

[ $[8,47], 123,[$ ] $]$

[ $[8,48], 483,[]]$

[ $[8,49], 539,[]]$

$[[8,50], 539,[]]$

[ $[9,1], 1,[]]$

$[[9,2], 10,[3]]$

$[[9,3], 10,[]]$

$[[9,4], 13,[]]$

$[[9,5], 28,[3]]$ 
$[[9,6], 31,[]]$

$[[9,7], 31,[3]]$

$[[9,8], 28,[]]$

$[[9,9], 28,[3]]$

$[[9,10], 70,[]]$

$[[9,11], 70,[3]]$

$[[9,12], 61,[]]$

$[[9,13], 40,[]]$

$[[9,14], 64,[3]]$

$[[9,15], 64,[]]$

$[[9,16], 34,[]]$

$[[9,17], 31,[]]$

$[[9,18], 70,[]]$

$[[9,19], 64,[]]$

$[[9,20], 61,[]]$

$[[9,21], 70,[]]$

$[[9,22], 40,[]]$

$[[9,23], 88,[3]]$

$[[9,24], 70,[]]$

$[[9,25], 40,[]]$

$[[9,26], 88,[]]$

$[[9,27], 64,[]]$

[ $[9,28], 40,[]]$

$[[9,29], 70,[]]$

$[[9,30], 70,[]]$

$[[9,31], 70,[]]$

$[[9,32], 232,[]]$

[ $[9,33], 1744,[]]$

[ $[9,34], 1744,[]]$

[ [ 10,1$], 1,[]]$

$[[10,2], 11,[]]$

$[[10,3], 13,[]]$

[ $[10,4], 13,[]]$

[ $[10,5], 31,[]]$

[ $[10,6], 53,[]]$

$[[10,7], 26,[2]]$

$[[10,8], 36,[]]$

$[[10,9], 63,[]]$

[ $[10,10], 63,[]]$

[ $[10,11], 31,[]]$

[ $[10,12], 31,[]]$

$[[10,13], 36,[]]$

$[[10,14], 36,[]]$

[ $[10,15], 51,[]]$

[ $[10,16], 51,[]]$

[ $[10,17], 83,[]]$

[ $[10,18], 83,[]]$

[ [ 10,19], 83, [ ] ]

[ $[10,20], 83,[]]$

[ [ 10,21] $, 63,[$ ] ]

$[[10,22], 31,[]]$

[ [ 10, 23 ], 51, [ ] ]

[ [ 10,24],61, [ ] ]

[ $[10,25], 61,[]]$

[ [ 10,26], 46, [ 2 ] ]

[ [ 10,27$], 83,[$ ] $]$

$[[10,28], 83,[]]$

[ [ 10,29],61, [ ] ]

[ [ 10,30], 91, [ ] ]

[ $[10,31], 67,[]]$

[ [ 10,32$], 46,[2]]$

$[[10,33], 83,[]]$ 
$[[10,34], 61,[]]$

$[[10,35], 91,[]]$

$[[10,36], 61,[]]$

$[[10,37], 61,[]]$

[ $[10,38], 61,[]]$

$[[10,39], 61,[]]$

$[[10,40], 83,[]]$

[ [ 10,41$], 83,[]]$

$[[10,42], 83,[]]$

$[[10,43], 83,[]]$

[ [ 10,44],378, [ ] ]

$[[10,45], 378,[]]$

[ [ 11,1$], 1,[]]$

$[[11,2], 12,[]]$

$[[11,3], 45,[]]$

$[[11,4], 100,[]]$

$[[11,5], 56,[]]$

$[[11,6], 100,[]]$

$[[11,7], 100,[]]$

$[[11,8], 100,[]]$

Example 5.2 (Computation of $H^{1}\left(G,\left[J_{G / H}\right]^{f l}\right)$ where $G=13 T m(1 \leq m \leq 9, m \neq 8,9), G=14 T m$ $(1 \leq m \leq 63, m \neq 62,63), G=15 T m(1 \leq m \leq 104, m \neq 103,104))$.

gap> Read("FlabbyResolutionFromBase.gap");

gap> for $m$ in [1..NrTransitiveGroups(13)-2] do

$>\mathrm{F}:=$ FlabbyResolutionLowRankFromGroup (Norm1TorusJ (13,m), TransitiveGroup (13,m)).actionF;

$>\operatorname{Print}([[n, i], \operatorname{Length}(F .1), F i l t e r e d(H 1(F), x->x>1)], " \backslash n ") ; \operatorname{od} ; \operatorname{Print}(" \backslash n ") ; o d$;

$[[13,1], 1,[]]$

$[[13,2], 14,[]]$

$[[13,3], 27,[]]$

$[[13,4], 40,[]]$

$[[13,5], 66,[]]$

$[[13,6], 144,[]]$

$[[13,7], 40,[]]$

gap> for $m$ in [1..NrTransitiveGroups(14)-2] do

$>\mathrm{F}:=$ FlabbyResolutionLowRankFromGroup (Norm1TorusJ $(14, \mathrm{~m}), \operatorname{TransitiveGroup}(14, \mathrm{~m}))$. actionF;

$>\operatorname{Print}([[14, \mathrm{~m}], \operatorname{Length}(\mathrm{F} .1), \operatorname{Filtered}(\mathrm{H} 1(\mathrm{~F}), \mathrm{x}->\mathrm{x}>1)], " \backslash \mathrm{n} ")$; od;

$[[14,1], 1,[]]$

$[[14,2], 15,[]]$

$[[14,3], 17,[]]$

$[[14,4], 31,[]]$

$[[14,5], 31,[]]$

$[[14,6], 50,[]]$

$[[14,7], 57,[]]$

[ [ 14,8 ], 101, [ ] ]

$[[14,9], 78,[]]$

$[[14,10], 64,[]]$

[ [ 14,11$], 86,[]]$

[ [ 14,12$], 115,[]]$

[ [ 14, 13 ], 115, [ ] ]

[ [ 14,14$], 129,[]]$

[ [ 14,15$], 129,[]]$

$[[14,16], 31,[]]$

[ [ 14,17$], 92,[]]$

[ [ 14, 18 ], 92, [ ] ]

[ [ 14, 19], 31, [ ] ]

[ [ 14, 20], 115, [ ] ]

[ [ 14,21$], 78,[]]$

[ [ 14, 22 ], 171, [ ] ]

[ [ 14, 23 ], 171, [ ] ]

[ [ 14, 24], 171, [ ] ] 
[ [ 14, 25 ], 171, [ ] ]

[ [ 14, 26 ], 129, [ ] ]

[ [ 14,27$], 99,[]]$

[ [ 14, 28 ], 99, [ ] ]

[ [ 14,29$], 78,[]]$

[ [ 14,30$], 92,[2]]$

$[[14,31], 171,[]]$

[ $[14,32], 171,[]]$

$[[14,33], 92,[]]$

[ [ 14,34], 92, [ ] ]

[ $[14,35], 92,[]]$

$[[14,36], 171,[]]$

$[[14,37], 171,[]]$

[ $[14,38], 99,[]]$

[ $[14,39], 183,[]]$

[ $[14,40], 127,[]]$

[ $[14,41], 127,[]]$

[ [ 14, 42 ], 92, [ ] ]

[ [ 14,43], 92, [ ] ]

$[[14,44], 99,[]]$

[ $[14,45], 171,[]]$

[ [ 14,46], 57, [ ] ]

[ [ 14,47 ], 57, [ ] ]

[ [ 14,48],127, [ ] ]

[ [ 14, 49 ], 57, [ ] ]

[ $[14,50], 92,[]]$

[ [ 14,51 ], 92, [ ] ]

[ [ 14,52 ], 129, [ ] ]

[ $[14,53], 127,[]]$

[ [ 14,54], 127, [ ] ]

[ $[14,55], 127,[]]$

$[[14,56], 127,[]]$

[ [ 14,57 ], 127, [ ] ]

[ $[14,58], 171,[]]$

[ [ 14,59], 171, [ ] ]

[ $[14,60], 171,[]]$

[ $[14,61], 171,[]]$

gap> for $m$ in [1..NrTransitiveGroups(15)-2] do

$>\mathrm{F}:=\mathrm{FlabbyResolutionLowRankFromGroup}(\operatorname{Norm} 1 \mathrm{TorusJ}(15, \mathrm{~m}), \operatorname{TransitiveGroup}(15, \mathrm{~m})$ ). actionF ;

$>\operatorname{Print}([[15, \mathrm{~m}], \operatorname{Length}(\mathrm{F} .1)$, Filtered $(\mathrm{H} 1(\mathrm{~F}), \mathrm{x}->\mathrm{x}>1)]$, "\n") ; od;

$[[15,1], 1,[]]$

$[[15,2], 16,[]]$

$[[15,3], 14,[]]$

$[[15,4], 21,[]]$

$[[15,5], 21,[]]$

$[[15,6], 39,[]]$

$[[15,7], 17,[]]$

$[[15,8], 36,[]]$

[ [ 15,9$], 79,[5]]$

$[[15,10], 36,[]]$

$[[15,11], 27,[]]$

[ [ 15, 12 ], 94, [ ] ]

$[[15,13], 82,[]]$

[ [ 15,14$], 97,[5]]$

[ [ 15, 15 ], 51, [ ] ]

$[[15,16], 36,[]]$

$[[15,17], 127,[]]$

[ [ 15, 18 ], 97, [ ] ]

[ [ 15, 19], 124, [ ] ]

[ [ 15, 20], 81, [ ] ]

[ $[15,21], 66,[]]$

$[[15,22], 39,[]]$ 
$[[15,23], 27,[]]$

$[[15,24], 36,[]]$

$[[15,25], 79,[]]$

[ $[15,26], 96,[]]$

[ [ 15,27], 127, [ ] ]

[ [ 15, 28 ], 81, [ ] ]

$[[15,29], 27,[]]$

$[[15,30], 94,[]]$

[ [ 15, 31], 169, [ ] ]

$[[15,32], 154,[]]$

[ $[15,33], 111,[]]$

$[[15,34], 186,[]]$

$[[15,35], 201,[]]$

$[[15,36], 96,[]]$

$[[15,37], 199,[]]$

[ [ 15, 38 ], 124, [ ] ]

[ [ 15, 39 ], 94, [ ] ]

[ $[15,40], 169,[]]$

[ [ 15,41], 186, [ ] ]

$[[15,42], 201,[]]$

[ [ 15,43], 201, [ ] ]

[ [ 15,44], 111, [ ] ]

[ $[15,45], 201,[]]$

[ $[15,46], 186,[]]$

[ $[15,47], 156,[]]$

$[[15,48], 169,[]]$

[ [ 15,49], 199, [ ] ]

$[[15,50], 94,[]]$

$[[15,51], 169,[]]$

[ [ 15,52], 201, [ ] ]

[ $[15,53], 456,[]]$

[ [ 15, 54 ], 201, [ ] ]

[ $[15,55], 201,[]]$

[ [ 15,56], 186, [ ] ]

[ [ 15, 57 ], 124, [ ] ]

[ $[15,58], 199,[]]$

[ [ 15, 59 ], 124, [ ] ]

$[[15,60], 169,[]]$

[ $[15,61], 471,[]]$

[ $[15,62], 471,[]]$

[ [ 15,63], 456, [ ] ]

[ $[15,64], 201,[]]$

[ [ 15,65], 199, [ ] ]

[ $[15,66], 199,[]]$

$[[15,67], 124,[]]$

[ [ 15,68 ], 199, [ ] ]

[ $[15,69], 456,[]]$

$[[15,70], 471,[]]$

[ [ 15,71], 111, [ ] ]

$[[15,72], 156,[]]$

[ [ 15,73], 199, [ ] ]

[ $[15,74], 199,[]]$

$[[15,75], 124,[]]$

[ [ 15,76], 471, [ ] ]

[ $[15,77], 471,[]]$

[ [ 15,78 ], 456, [ ] ]

[ [ 15,79], 201, [ ] ]

[ [ 15,80], 201, [ ] ]

$[[15,81], 111,[]]$

[ [ 15,82 ], 199, [ ] ]

[ $[15,83], 471,[]]$

[ [ 15,84], 201, [ ] ]

[ $[15,85], 201,[]]$ 
[ [ 15,86 ], 201, [ ] ]

[ [ 15,87 ], 201, [ ] ]

[ [ 15,88$], 471,[]]$

[ [ 15,89$], 471,[$ ] ]

[ [ 15,90$], 471,[]]$

[ [ 15, 91$], 471,[]]$

[ [ 15,92$], 124,[]]$

[ [ 15,93$], 471,[]]$

[ [ 15, 94$], 199,[]]$

[ [ 15,95 ], 124, [ ] ]

[ [ 15,96], 199, [ ] ]

[ [ 15,97 ], 199, [ ] ]

[ [ 15,98$], 124,[]]$

[ [ 15, 99$], 199,[]]$

$[[15,100], 199,[]]$

[ [ 15,101$], 124,[]]$

[ [ 15, 102], 199, [ ] ]

Example 5.3 (Computation of $H^{1}\left(G,\left[J_{G / H}\right]^{f l}\right)=0$ where $G=12 T 295 \simeq M_{12}$ and $\left.G=22 T 38 \simeq M_{22}\right)$.

gap> Read("FlabbyResolutionFromBase.gap");

gap> G:=TransitiveGroup $(12,295)$;

M(12)

gap> F:=FlabbyResolutionLowRankFromGroup (Norm1Torus J $(12,295)$, G) . actionF ;

<matrix group with 2 generators>

gap> $[[12,295], \operatorname{Length}(F .1), F i l t e r e d(H 1(F), x->x>1)]$;

$[[12,295], 814,[]]$

gap> G:=TransitiveGroup $(22,38)$;

t22n38

gap> StructureDescription(G) ;

"M22"

gap> F:=FlabbyResolutionLowRankFromGroup(Norm1Torus J $(22,38)$, G) . actionF ;

<matrix group with 2 generators>

gap> $[[22,38]$, Length $(F .1)$, Filtered $(H 1(F), x->x>1)]$;

[ [ 22, 38 ], 672, [ ] ]

\section{Proof of Theorem 1.18}

Let $k$ be a number field, $K / k$ be a finite extension, $\mathbb{A}_{K}^{\times}$be the idele group of $K$ and $L / k$ be the Galois closure of $K / k$. Let $G=\operatorname{Gal}(L / k)=n T m$ be a transitive subgroup of $S_{n}$ and $H=\operatorname{Gal}(L / K)$ with $[G: H]=n$.

For $x, y \in G$, we denote $[x, y]=x^{-1} y^{-1} x y$ the commutator of $x$ and $y$, and $[G, G]$ the commutator group of $G$. Let $V_{k}$ be the set of all places of $k$ and $G_{v}$ be the decomposition group of $G$ at $v \in V_{k}$.

Definition 6.1 (Drakokhrust and Platonov PD85a, page 350], [DP87, page 300]). Let $k$ be a number field, $L \supset K \supset k$ be a tower of finite extensions where $L$ is normal over $k$.

We call the group

$$
\operatorname{Obs}(K / k)=\left(N_{K / k}\left(\mathbb{A}_{K}^{\times}\right) \cap k^{\times}\right) / N_{K / k}\left(K^{\times}\right)
$$

the total obstruction to the Hasse norm principle for $K / k$ and

$$
\operatorname{Obs}_{1}(L / K / k)=\left(N_{K / k}\left(\mathbb{A}_{K}^{\times}\right) \cap k^{\times}\right) /\left(\left(N_{L / k}\left(\mathbb{A}_{L}^{\times}\right) \cap k^{\times}\right) N_{K / k}\left(K^{\times}\right)\right)
$$

the first obstruction to the Hasse norm principle for $K / k$ corresponding to the tower $L \supset K \supset k$.

Note that (i) $\operatorname{Obs}(K / k)=1$ if and only if the Hasse norm principle holds for $K / k$; and (ii) $\operatorname{Obs}_{1}(L / K / k)=$ $\operatorname{Obs}(K / k) /\left(N_{L / k}\left(\mathbb{A}_{L}^{\times}\right) \cap k^{\times}\right)$.

Drakokhrust and Platonov gave a formula for computing the first obstruction $\operatorname{Obs}_{1}(L / K / k)$ :

Theorem 6.2 (Drakokhrust and Platonov [PD85a, page 350], PD85b, pages 789-790], [DP87, Theorem 1]). Let $k$ be a number field, $L \supset K \supset k$ be a tower of finite extensions where $L$ is normal over $k$. Let $G=\operatorname{Gal}(L / k)$ and $H=\operatorname{Gal}(L / K)$. Then

$$
\operatorname{Obs}_{1}(L / K / k) \simeq \operatorname{Ker} \psi_{1} / \varphi_{1}\left(\operatorname{Ker} \psi_{2}\right)
$$


where in the the commutative diagram

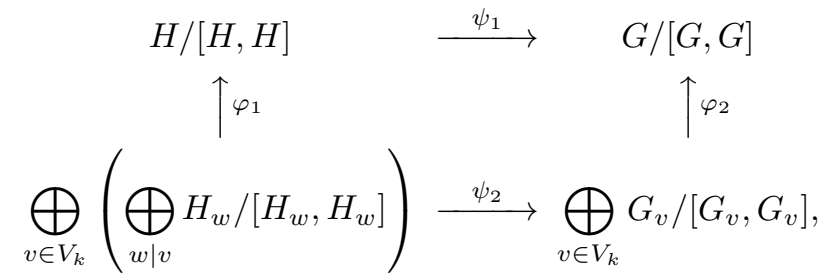

$\psi_{1}, \varphi_{1}$ and $\varphi_{2}$ are defined by the inclusions $H \subset G, H_{w} \subset H$ and $G_{v} \subset G$ respectively, and

$$
\psi_{2}\left(h\left[H_{w}, H_{w}\right]\right)=x^{-1} h x\left[G_{v}, G_{v}\right]
$$

for $h \in H_{w}=H \cap x^{-1} h x\left[G_{v}, G_{v}\right](x \in G)$.

Let $\psi_{2}^{v}$ be the restriction of $\psi_{2}$ to the subgroup $\bigoplus_{w \mid v} H_{w} /\left[H_{w}, H_{w}\right]$ with respect to $v \in V_{k}$ and $\psi_{2}^{\mathrm{nr}}$ (resp. $\psi_{2}^{\mathrm{r}}$ ) be the restriction of $\psi_{2}$ to the unramified (resp. the ramified) places $v$ of $k$.

Proposition 6.3 (Drakokhrust and Platonov [DP87]). Let $k, L \supset K \supset k, G$ and $H$ be as in Theorem 6.2. (i) ([DP87, Lemma 1]) Places $w_{i} \mid v$ of $K$ are in one-to-one correspondence with the set of double cosets in the decomposition $G=\cup_{i=1}^{r_{v}} H x_{i} G_{v}$ where $H_{w_{i}}=H \cap x_{i} G_{v} x_{i}^{-1}$;

(ii) ([DP87, Lemma 2]) If $G_{v_{1}} \leq G_{v_{2}}$, then $\varphi_{1}\left(\operatorname{Ker} \psi_{2}^{v_{1}}\right) \subset \varphi_{1}\left(\operatorname{Ker} \psi_{2}^{v_{2}}\right)$;

(iii) $\left(\left[\mathrm{DP} 87\right.\right.$, Theorem 2]) $\varphi_{1}\left(\operatorname{Ker} \psi_{2}^{\mathrm{nr}}\right)=\Phi^{G}(H) /[H, H]$ where $\Phi^{G}(H)=\left\langle[h, x] \mid h \in H \cap x H x^{-1}, x \in G\right\rangle$;

(iv) ([DP87, Lemma 8]) If $[K: k]=p^{r}(r \geq 1)$ and $\operatorname{Obs}\left(K_{p} / k_{p}\right)=1$ where $k_{p}=L^{G_{p}}, K_{p}=L^{H_{p}}, G_{p}$ and $H_{p} \leq H \cap G_{p}$ are $p$-Sylow subgroups of $G$ and $H$ respectively, then $\operatorname{Obs}(K / k)=1$.

Remark 6.4. The inverse direction of Proposition 6.3 (iv) does not hold in general. For example, if $n=8$, $G=8 T 13 \simeq A_{4} \times C_{2}$ and there exists a place $v$ of $k$ such that $G_{v} \simeq V_{4}$, then $\operatorname{Obs}(K / k)=1$ but $G_{2}=8 T 3 \simeq\left(C_{2}\right)^{3}$ and $\operatorname{Obs}\left(K_{2} / k_{2}\right) \neq 1$ may occur (see Theorem 1.18 and Table 2).

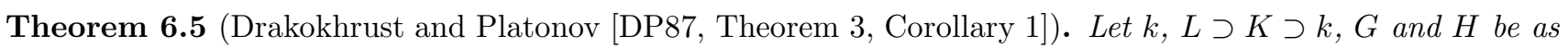
in Theorem 6.2. Let $H_{i} \leq G_{i} \leq G(1 \leq i \leq m), H_{i} \leq H \cap G_{i}, k_{i}=L^{G_{i}}$ and $K_{i}=L^{H_{i}}$. If Obs $\left(K_{i} / k_{i}\right)=1$ for all $1 \leq i \leq m$ and

$$
\bigoplus_{i=1}^{m} \widehat{H}^{-3}\left(G_{i}, \mathbb{Z}\right) \stackrel{\text { cores }}{\longrightarrow} \widehat{H}^{-3}(G, \mathbb{Z})
$$

is surjective, then $\operatorname{Obs}(K / k)=\operatorname{Obs}_{1}(L / K / k)$. In particular, if $[K: k]=n$ is square-free, then $\mathrm{Obs}(K / k)=$ $\operatorname{Obs}_{1}(L / K / k)$.

We note that if $L / k$ is an unramified extension, then $A(T)=0$ and $H^{1}\left(G,\left[J_{G / H}\right]^{f l}\right) \simeq \amalg(T) \simeq \mathrm{Obs}(K / k)$ where $T=R_{K / k}^{(1)}\left(\mathbb{G}_{m}\right)$ (see Theorem 1.2 and Theorem 1.9). If, in addition, $\operatorname{Obs}(K / k)=\operatorname{Obs}_{1}(L / K / k)(\mathrm{e} . \mathrm{g}$. $[K: k]=6,10,14,15$; square-free, see Theorem 6.5), then $\operatorname{Obs}(K / k)=\operatorname{Obs}_{1}(L / K / k)=\operatorname{Ker} \psi_{1} / \varphi_{1}\left(\operatorname{Ker} \psi_{2}^{\mathrm{nr}}\right) \simeq$ $\operatorname{Ker} \psi_{1} /\left(\Phi^{G}(H) /[H, H]\right.$ ) (see Proposition 6.3 (iii)).

Theorem 6.6 (Drakokhrust Dra89, Theorem 1], see also Opolka Opo80, Satz 3] for the existence of $\widetilde{L}$ ). Let $k, L \supset K \supset k, G$ and $H$ be as in Theorem 6.2. Assume that $\widetilde{L} \supset L \supset k$ is a tower of Galois extensions with $\widetilde{G}=\operatorname{Gal}(\widetilde{L} / k)$ and $\widetilde{H}=\operatorname{Gal}(\widetilde{L} / K)$ which correspond to a central extension $1 \rightarrow A \rightarrow \widetilde{G} \rightarrow G \rightarrow 1$ with $A \cap[\widetilde{G}, \widetilde{G}] \simeq M(G)=H^{2}\left(G, \mathbb{C}^{\times}\right)$; the Schur multiplier of $G$ (this is equivalent to the inflation $M(G) \rightarrow M(\widetilde{G})$ being the zero map, see Beyl and Tappe [BT82, Proposition 2.13, page 85]). Then $\operatorname{Obs}(K / k)=\mathrm{Obs}_{1}(\widetilde{L} / K / k)$. In particular, if $\widetilde{G}$ is a Schur cover of $G$, i.e. $A \simeq M(G)$, then $\operatorname{Obs}(K / k)=\operatorname{Obs}_{1}(\widetilde{L} / K / k)$.

Indeed, Drakokhrust [Dra89, Theorem 1] shows that $\operatorname{Obs}(K / k) \simeq \operatorname{Ker} \widetilde{\psi}_{1} / \widetilde{\varphi}_{1}\left(\operatorname{Ker} \widetilde{\psi}_{2}\right)$ where the maps $\widetilde{\psi}_{1}, \widetilde{\psi}_{2}$ and $\widetilde{\varphi}_{1}$ are defined as in [Dra89, page 31, the paragraph before Proposition 1]. The proof of [Dra89, Proposition 1] shows that this group is the same as $\operatorname{Obs}_{1}(\widetilde{L} / K / k)$ (see also [Dra89, Lemma 2, Lemma 3 and Lemma 4]).

We made the following functions of GAP (GAP) which will be used in the proof of Theorem 1.18

FirstObstructionN $(G, H)$. ker returns the list $\left[l_{1},\left[l_{2}, l_{3}\right]\right]$ where $l_{1}$ is the abelian invariant of the numerator of the first obstruction $\operatorname{Ker} \psi_{1}=\left\langle y_{1}, \ldots, y_{t}\right\rangle$ with respect to $G, H$ as in Theorem $6.2, l_{2}=\left[e_{1}, \ldots, e_{m}\right]$ is the abelian invariant of $H^{a b}=H /[H, H]=\left\langle x_{1}, \ldots, x_{m}\right\rangle$ with $e_{i}=\operatorname{order}\left(x_{i}\right)$ and $l_{3}=\left[l_{3,1}, \ldots, l_{3, t}\right], l_{3, i}=\left[r_{i, 1}, \ldots, r_{i, m}\right]$ is the list with $y_{i}=x_{1}^{r_{i, 1}} \cdots x_{m}^{r_{i, m}}$ for $H \leq G \leq S_{n}$.

FirstObstructionN $(G)$. ker returns the same as FirstobstructionN $(G, H)$. ker where $H=\operatorname{Stab}_{1}(G)$ is the stabilizer of 1 in $G \leq S_{n}$. 
FirstObstructionDnr $(G, H)$.Dnr returns the list $\left[l_{1},\left[l_{2}, l_{3}\right]\right]$ where $l_{1}$ is the abelian invariant of the unramified part of the denominator of the first obstruction $\varphi_{1}\left(\operatorname{Ker} \psi_{2}^{\mathrm{nr}}\right)=\Phi^{G}(H) /[H, H]=\left\langle y_{1}, \ldots, y_{t}\right\rangle$ with respect to $G$, $H$ as in Proposition 6.3 (iii), $l_{2}=\left[e_{1}, \ldots, e_{m}\right]$ is the abelian invariant of $H^{a b}=H /[H, H]=\left\langle x_{1}, \ldots, x_{m}\right\rangle$ with $e_{i}=\operatorname{order}\left(x_{i}\right)$ and $l_{3}=\left[l_{3,1}, \ldots, l_{3, t}\right], l_{3, i}=\left[r_{i, 1}, \ldots, r_{i, m}\right]$ is the list with $y_{i}=x_{1}^{r_{i, 1}} \cdots x_{m}^{r_{i, m}}$ for $H \leq G \leq S_{n}$.

FirstObstructionDnr $(G)$. Dnr returns the same as FirstObstructionDnr $(G, H)$. Dnr where $H=\operatorname{Stab}_{1}(G)$ is the stabilizer of 1 in $G \leq S_{n}$.

FirstObstructionDr $\left(G, G_{v}, H\right)$.Dr returns the list $\left[l_{1},\left[l_{2}, l_{3}\right]\right]$ where $l_{1}$ is the abelian invariant of the ramified part of the denominator of the first obstruction $\varphi_{1}\left(\operatorname{Ker} \psi_{2}^{v}\right)=\left\langle y_{1}, \ldots, y_{t}\right\rangle$ with respect to $G, G_{v}, H$ as in Theorem 6.2, $l_{2}=\left[e_{1}, \ldots, e_{m}\right]$ is the abelian invariant of $H^{a b}=H /[H, H]=\left\langle x_{1}, \ldots, x_{m}\right\rangle$ with $e_{i}=\operatorname{order}\left(x_{i}\right)$ and $l_{3}=\left[l_{3,1}, \ldots, l_{3, t}\right], l_{3, i}=\left[r_{i, 1}, \ldots, r_{i, m}\right]$ is the list with $y_{i}=x_{1}^{r_{i, 1}} \cdots x_{m}^{r_{i, m}}$ for $G_{v}, H \leq G \leq S_{n}$.

FirstObstructionDr $\left(G, G_{v}\right)$. Dr returns the same as FirstobstructionDr $\left(G, G_{v}, H\right)$. Dr where $H=\operatorname{Stab}_{1}(G)$ is the stabilizer of 1 in $G \leq S_{n}$.

SchurCoverG $(G)$. SchurCover (resp. SchurCoverG $(G)$.epi) returns one of the Schur covers $\widetilde{G}$ of $G$ (resp. the surjective map $\pi$ ) in a central extension $1 \rightarrow A \rightarrow \widetilde{G} \stackrel{\pi}{\rightarrow} G \rightarrow 1$ with $A \simeq M(G)$; Schur multiplier of $G$ (see Karpilovsky Kap87, page 16]). The Schur covers $\widetilde{G}$ are stem extensions, i.e. $A \leq Z(\widetilde{G}) \cap[\widetilde{G}, \widetilde{G}]$, of the maximal size. This function is based on the built-in function EpimorphismSchurCover in GAP.

MinimalStemExtensions $(G)[j]$. MinimalStemExtension (resp. MinimalStemExtensions $(G)[j]$.epi) returns the $j$-th minimal stem extension $\bar{G}=\widetilde{G} / A^{\prime}$, i.e. $\bar{A} \leq Z(\bar{G}) \cap[\bar{G}, \bar{G}]$, of $G$ provided by the Schur cover $\widetilde{G}$ of $G$ via SchurCoverG $(G)$. SchurCover where $A^{\prime}$ is the $j$-th maximal subgroup of $A=M(G)$ (resp. the surjective map $\bar{\pi}$ ) in the commutative diagram

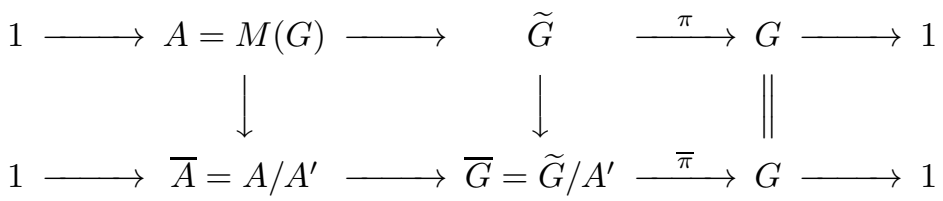

(see Robinson [Rob96, Exercises 11.4]). This function is based on the built-in function EpimorphismSchurCover in GAP.

ResolutionNormalSeries (LowerCentralSeries $(G), n+1$ ) (resp. ResolutionNormalSeries (DerivedSeries $(G), n+1)$, ResolutionFiniteGroup $(G, n+1)$ ) returns a free resolution $R G$ of $G$ when $G$ is nilpotent (resp. solvable, finite). This function is the built-in function of HAP ([HAP) in GAP (GAP]) .

ResHnZ ( $R G, R H, n)$. HnGZ (resp. ResHnZ $(R G, R H, n)$. HnHZ) returns the abelian invariants of $H^{n}(G, \mathbb{Z})$ (resp. $H^{n}(H, \mathbb{Z})$ ) with respect to Smith normal form, for free resolutions $R G$ and $R H$ of $G$ and $H$ respectively.

$\operatorname{ResHnZ}(R G, R H, n)$. Res returns the list $L=\left[l_{1}, \ldots, l_{s}\right]$ where $H^{n}(G, \mathbb{Z})=\left\langle x_{1}, \ldots, x_{s}\right\rangle \stackrel{\text { res }}{\longrightarrow} H^{n}(H, \mathbb{Z})=$ $\left\langle y_{1}, \ldots, y_{t}\right\rangle, \operatorname{res}\left(x_{i}\right)=\prod_{j=1}^{t} y_{j}^{l_{i, j}}$ and $l_{i}=\left[l_{i, 1}, \ldots, l_{i, t}\right]$ for free resolutions $R G$ and $R H$ of $G$ and $H$ respectively.

$\operatorname{ResHnZ}(R G, R H, n)$. Ker returns the list $L=\left[l_{1},\left[l_{2}, l_{3}\right]\right]$ where $l_{1}$ is the abelian invariant of $\operatorname{Ker}\left\{H^{n}(G, \mathbb{Z}) \stackrel{\text { res }}{\longrightarrow}\right.$ $\left.H^{n}(H, \mathbb{Z})\right\}=\left\langle y_{1}, \ldots, y_{t}\right\rangle, l_{2}=\left[d_{1}, \ldots, d_{s}\right]$ is the abelian invariant of $H^{n}(G, \mathbb{Z})=\left\langle x_{1}, \ldots, x_{s}\right\rangle$ with $d_{i}=\operatorname{ord}\left(x_{i}\right)$ and $l_{3}=\left[l_{3,1}, \ldots, l_{3, t}\right], l_{3, j}=\left[r_{j, 1}, \ldots, r_{j, s}\right]$ is the list with $y_{j}=x_{1}^{r_{j, 1}} \cdots x_{s}^{r_{j, s}}$ for free resolutions $R G$ and $R H$ of $G$ and $H$ respectively.

$\operatorname{ResHnZ}(R G, R H, n)$. Coker returns the list $L=\left[l_{1},\left[l_{2}, l_{3}\right]\right]$ where $l_{1}=\left[e_{1}, \ldots, e_{t}\right]$ is the abelian invariant of $\operatorname{Coker}\left\{H^{n}(G, \mathbb{Z}) \stackrel{\text { res }}{\longrightarrow} H^{n}(H, \mathbb{Z})\right\}=\left\langle\overline{y_{1}}, \ldots, \overline{y_{t}}\right\rangle$ with $e_{j}=\operatorname{ord}\left(\overline{y_{j}}\right), l_{2}=\left[d_{1}, \ldots, d_{s}\right]$ is the abelian invariant of $H^{n}(H, \mathbb{Z})=\left\langle x_{1}, \ldots, x_{s}\right\rangle$ with $d_{i}=\operatorname{ord}\left(x_{i}\right)$ and $l_{3}=\left[l_{3,1}, \ldots, l_{3, t}\right], l_{3, j}=\left[r_{j, 1}, \ldots, r_{j, s}\right]$ is the list with $\overline{y_{j}}={\overline{x_{1}}}^{r j, 1} \ldots{\overline{x_{s}}}^{r, s}$ for free resolutions $R G$ and $R H$ of $G$ and $H$ respectively.

$\operatorname{KerResH3Z}(G, H)$ returns the list $L=\left[l_{1},\left[l_{2}, l_{3}\right]\right]$ where $l_{1}$ is the abelian invariant of $\operatorname{Ker}\left\{H^{3}(G, \mathbb{Z}) \stackrel{\text { res }}{\longrightarrow}\right.$ $\left.\oplus_{i=1}^{m^{\prime}} H^{3}\left(G_{i}, \mathbb{Z}\right)\right\}=\left\langle y_{1}, \ldots, y_{t}\right\rangle$ where $H_{i} \leq G_{i} \leq G, H_{i} \leq H \cap G_{i},\left[G_{i}: H_{i}\right]=n$ and the action of $G_{i}$ on $\mathbb{Z}\left[G_{i} / H_{i}\right]$ may be regarded as $n T m(n \leq 15, n \neq 12)$ which is not in Table $1, l_{2}=\left[d_{1}, \ldots, d_{s}\right]$ is the abelian invariant of $H^{3}(G, \mathbb{Z})=\left\langle x_{1}, \ldots, x_{s}\right\rangle$ with $d_{i^{\prime}}=\operatorname{ord}\left(x_{i^{\prime}}\right)$ and $l_{3}=\left[l_{3,1}, \ldots, l_{3, t}\right], l_{3, j}=\left[r_{j, 1}, \ldots, r_{j, s}\right]$ is the list with $y_{j}=x_{1}^{r_{j, 1}} \cdots x_{s}^{r_{j, s}}$ for groups $G$ and $H$ (cf. Theorem 6.6). 
The functions above are available from

https://www.math.kyoto-u.ac.jp/ yamasaki/Algorithm/Norm1ToriHNP.

\section{Proof of Theorem 1.18}

Let $G=\operatorname{Gal}(L / k)=n T m \leq S_{n}$ be the $m$-th transitive subgroup of $S_{n}$ and $H=\operatorname{Gal}(L / K) \leq G$ with $[G: H]=n$. Let $V_{k}$ be the set of all places of $k$ and $G_{v}$ be the decomposition group of $G$ at $v \in V_{k}$.

We split the proof into the following cases:

(1) $G=8 \mathrm{Tm}(m=2,3,4,13,14,21,31,37,38)$,

(2) $G=8 T m(m=9,11,15,19,22,32)$,

(3) $G=9 \mathrm{Tm}(m=2,5,7,9,11,14,23)$,

(4) $G=10 T m(m=7,26,32)$,

(5) $G=14 T 30$,

(6) $G=15 T m(m=9,14)$.

For the reader's convenience, we also give the GAP computations to the known cases: $G=4 T 2 \simeq V_{4}, 4 T 4 \simeq$ $A_{4}, 6 T 4 \simeq A_{4}, 6 T 12 \simeq A_{5}$ (see Example 6.9 and Example 6.10).

In order to prove the statement of the theorem, we may assume that $H=\operatorname{Stab}_{1}(G)$ is the stabilizer of 1 in $G$, i.e. $L=k\left(\theta_{1}, \ldots, \theta_{n}\right)$ and $K=L^{H}=k\left(\theta_{1}\right)$, without loss of generality except for the cases (2) $G=8 T m$ $(m=9,11,15,19,22,32)$ and $G=10 T 32 \simeq S_{6}$ because the center $Z(G)$ and the commutator group $\left[G^{\prime}, G^{\prime}\right]$ where $G^{\prime} \leq G$ is a characteristic subgroup in the statement of the theorem, are characteristic subgroups of $G$, i.e. invariants under the automorphisms of $G$.

For the cases (2) $G=8 \mathrm{Tm}$, by the assumption of the statement of the theorem, we may assume that $H=\operatorname{Stab}_{1}(G)$ is the stabilizer of 1 in $G$ because (the multi-set) $\left\{\operatorname{Orb}_{G^{\prime}}(i) \mid 1 \leq i \leq n\right\}\left(G^{\prime} \leq G\right)$ is invariant under the conjugacy actions of $G$, i.e. inner automorphisms of $G$.

For the case $G=10 T 32 \simeq S_{6}$, there exist exactly 10 subgroups $H \leq G$ with $[G: H]=10$ which are conjugate in $G$. Hence we may assume that $H=\operatorname{Stab}_{1}(G)$ without loss of generality.

By Theorem 1.2 and Theorem 1.15 it is enough to give a necessary and sufficient condition for $\amalg(T)=0$.

(1) $n=8: G=8 \mathrm{Tm}(m=2,3,4,13,14,21,31,37,38)$. Applying the functions FirstObstructionN $(G)$ and FirstObstructionDnr $(G)$, we have $\operatorname{Obs}_{1}(L / K / k)=1$ except for $G=8 T 21 \simeq\left(C_{2}\right)^{3} \rtimes C_{4}$. For $G=8 T 21$, we obtain that $\operatorname{Obs}_{1}(L / K / k) \simeq \mathbb{Z} / 2 \mathbb{Z}$.

(1-1) The case $G=8 T 3 \simeq\left(C_{2}\right)^{3}$. This case follows from Theorem 1.8 because $H=1$. See also Example 6.11 and the second paragraph after Theorem 1.8 .

(1-2) The case $G=8 T 21 \simeq\left(C_{2}\right)^{3} \rtimes C_{4}$. We have $H=H^{a b} \simeq C_{2} \times C_{2}$. Applying FirstObstructionN $(G)$ and FirstObstructionDnr $(G)$, we obtain that $\operatorname{Ker} \psi_{1} / \varphi_{1}\left(\operatorname{Ker} \psi_{2}^{\mathrm{nr}}\right) \simeq \mathbb{Z} / 2 \mathbb{Z}$. By Theorem 1.15, we get $\operatorname{Obs}(K / k)=$ $\operatorname{Obs}_{1}(L / K / k)$ when $L / k$ is unramified (see the paragraph after Theorem 6.5). Use Theorem 6.5. Applying the function $\operatorname{KerResH3Z}(\mathrm{G}, \mathrm{H})$, we see that $\operatorname{Ker}\left\{H^{3}(G, \mathbb{Z}) \stackrel{\text { res }}{\longrightarrow} \oplus_{i=1}^{m^{\prime}} H^{3}\left(G_{i}, \mathbb{Z}\right)\right\}=0$ and hence $\oplus_{i=1}^{m^{\prime}} \widehat{H}^{-3}\left(G_{i}, \mathbb{Z}\right) \stackrel{\text { cores }}{\longrightarrow}$ $\widehat{H}^{-3}(G, \mathbb{Z})$ is surjective. It follows from Theorem 6.5 that $\operatorname{Obs}(K / k)=\operatorname{Obs}_{1}(L / K / k)$. Applying the function FirstObstructionDr $\left(G, G^{\prime}\right)$ for all subgroups $G^{\prime} \leq G$, we find that $\operatorname{Obs}_{1}(L / K / k)=1$ if and only if there exists $v \in V_{k}$ such that $G_{v}=G$ (see Example 6.11).

(1-3) The case $G=8 T m(m=2,4,13,14,37)$. Because $\operatorname{Obs}_{1}(K / k)=1$, we just apply Theorem 6.6. We have the Schur multiplier $M(G) \simeq \mathbb{Z} / 2 \mathbb{Z}$ for $G=8 T m(m=2,4,13,14,37)$.

(1-3-1) The case $G=8 T 2 \simeq C_{4} \times C_{2}$ (see also Theorem 1.8 because $H=1$ ). Apply Theorem 6.6. We obtain a Schur cover $1 \rightarrow M(G) \simeq \mathbb{Z} / 2 \mathbb{Z} \rightarrow \widetilde{G} \stackrel{\pi}{\rightarrow} G \rightarrow 1$ with $\widetilde{G} \simeq\left(C_{4} \times C_{2}\right) \rtimes C_{2}, \widetilde{H} \simeq C_{2}$ and Obs $(K / k)=$ $\operatorname{Obs}_{1}(\widetilde{L} / K / k)$. By Theorem 1.15, $\operatorname{Ker} \widetilde{\psi}_{1} / \widetilde{\varphi}_{1}\left(\operatorname{Ker} \widetilde{\psi}_{2}^{\text {nr }}\right) \simeq \mathbb{Z} / 2 \mathbb{Z}$ (see the paragraph after Theorem 6.5). By applying FirstObstructionDr $\left(\widetilde{G}, \widetilde{G}^{\prime}, \widetilde{H}\right)$ for all subgroups $\widetilde{G}^{\prime} \leq \widetilde{G}$, we obtain that $\operatorname{Obs}_{1}(\widetilde{L} / K / k)=1$ if and only if there exists $v \in V_{k}$ such that $\widetilde{G}_{v}=\widetilde{G}$ if and only if there exists $v \in V_{k}$ such that $G_{v}=G$ (see Example 6.11).

(1-3-2) The case $G=8 T 4 \simeq D_{4}$ (see also Theorem 1.8 because $H=1$ ). Apply Theorem 6.6 We obtain a Schur cover $1 \rightarrow M(G) \simeq \mathbb{Z} / 2 \mathbb{Z} \rightarrow \widetilde{G} \stackrel{\pi}{\rightarrow} G \rightarrow 1$ with $\widetilde{G} \simeq D_{8}, \widetilde{H} \simeq C_{2}$ and $\operatorname{Obs}(K / k)=\operatorname{Obs}_{1}(\widetilde{L} / K / k)$. By Theorem 1.15, Ker $\widetilde{\psi}_{1} / \widetilde{\varphi}_{1}\left(\operatorname{Ker} \widetilde{\psi}_{2}^{\mathrm{nr}}\right) \simeq \mathbb{Z} / 2 \mathbb{Z}$. By applying FirstObstructionDr $\left(\widetilde{G}, \widetilde{G}^{\prime}, \widetilde{H}\right)$ for all subgroups $\widetilde{G}^{\prime} \leq \widetilde{G}$, we obtain that $\operatorname{Obs}_{1}(\widetilde{L} / K / k)=1$ if and only if there exists $v \in V_{k}$ such that $D_{4} \leq \widetilde{G}_{v}$ if and only if there exists $v \in V_{k}$ such that $V_{4} \leq G_{v}$ (see Example 6.11).

(1-3-3) The case $G=8 T 13 \simeq A_{4} \times C_{2}$. We have $H \simeq C_{3}$. Apply Theorem 6.6. We obtain a Schur cover $1 \rightarrow M(G) \simeq \mathbb{Z} / 2 \mathbb{Z} \rightarrow \widetilde{G} \stackrel{\pi}{\rightarrow} G \rightarrow 1$ with $\widetilde{G} \simeq\left(\left(C_{4} \times C_{2}\right) \rtimes C_{2}\right) \rtimes C_{3}, \widetilde{H} \simeq C_{6}$ and $\operatorname{Obs}(K / k)=\operatorname{Obs}_{1}(\widetilde{L} / K / k)$. By Theorem 1.15, Ker $\widetilde{\psi}_{1} / \widetilde{\varphi}_{1}\left(\operatorname{Ker} \widetilde{\psi}_{2}^{\text {nr }}\right) \simeq \mathbb{Z} / 2 \mathbb{Z}$. By applying FirstObstructionDr $\left(\widetilde{G}, \widetilde{G}^{\prime}, \widetilde{H}\right)$ for all subgroups $\widetilde{G}^{\prime} \leq \widetilde{G}$, we obtain that $\operatorname{Obs}_{1}(\widetilde{L} / K / k)=1$ if and only if there exists $v \in V_{k}$ such that $V_{4} \leq G_{v}$ (see Example 6.11). 
(1-3-4) The case $G=8 T 14 \simeq S_{4}$. We have $H \simeq C_{3}$. Apply Theorem 6.6. We obtain a Schur cover $1 \rightarrow$ $M(G) \simeq \mathbb{Z} / 2 \mathbb{Z} \rightarrow \widetilde{G} \stackrel{\pi}{\rightarrow} G \rightarrow 1$ with $\widetilde{G} \simeq G L_{2}\left(\mathbb{F}_{3}\right)$ and $\widetilde{H} \simeq C_{6}$. By Theorem 1.15. Ker $\widetilde{\psi}_{1} / \widetilde{\varphi}_{1}\left(\right.$ Ker $\left.\widetilde{\psi}_{2}^{\text {nr }}\right) \simeq \mathbb{Z} / 2 \mathbb{Z}$. By applying FirstObstructionDr $\left(\widetilde{G}, \widetilde{G}^{\prime}, \widetilde{H}\right)$ for all subgroups $\widetilde{G}^{\prime} \leq \widetilde{G}$, we obtain that $\operatorname{Obs}_{1}(\widetilde{L} / K / k)=1$ if and only if there exists $v \in V_{k}$ such that $V_{4} \leq G_{v}$ (see Example 6.11).

(1-3-5) The case $G=8 T 37 \simeq \mathrm{PSL}_{3}\left(\mathbb{F}_{2}\right) \simeq \mathrm{PSL}_{2}\left(\mathbb{F}_{7}\right)$. We have $H \simeq C_{7} \rtimes C_{3}$. We obtain a Schur cover $1 \rightarrow M(G) \simeq \mathbb{Z} / 2 \mathbb{Z} \rightarrow \widetilde{G} \stackrel{\pi}{\rightarrow} G \rightarrow 1$ with $\widetilde{G} \simeq \mathrm{SL}_{2}\left(\mathbb{F}_{7}\right), \widetilde{H} \simeq C_{2} \times\left(C_{7} \rtimes C_{3}\right)$ and $\operatorname{Obs}(K / k)=\operatorname{Obs}_{1}(\widetilde{L} / K / k)$. By Theorem 1.15, Ker $\widetilde{\psi}_{1} / \widetilde{\varphi}_{1}\left(\operatorname{Ker} \widetilde{\psi}_{2}^{\mathrm{nr}}\right) \simeq \mathbb{Z} / 2 \mathbb{Z}$. By applying First0bstructionDr $\left(\widetilde{G}, \widetilde{G}^{\prime}, \widetilde{H}\right)$ for all subgroups $\widetilde{G}^{\prime} \leq \widetilde{G}$, we obtain that $\operatorname{Obs}_{1}(\widetilde{L} / K / k)=1$ if and only if there exists $v \in V_{k}$ such that $V_{4} \leq G_{v}$ (see Example 6.11).

(1-4) The case $G=8 \mathrm{Tm}(m=31,38)$. Applying FirstObstructionN $(G)$ and FirstObstructionDnr $(G)$, we have $\operatorname{Obs}_{1}(L / K / k)=1$. For $G=8 T 31$ (resp. $\left.G=8 T 38\right)$, we have $M(G) \simeq(\mathbb{Z} / 2 \mathbb{Z})^{\oplus 4}\left(\right.$ resp. $\left.M(G) \simeq(\mathbb{Z} / 2 \mathbb{Z})^{\oplus 2}\right)$. Hence we take a minimal stem extension $\bar{G}=\widetilde{G} / A^{\prime}$, i.e. $\bar{A} \leq Z(\bar{G}) \cap[\bar{G}, \bar{G}]$, of $G$ in the commutative diagram

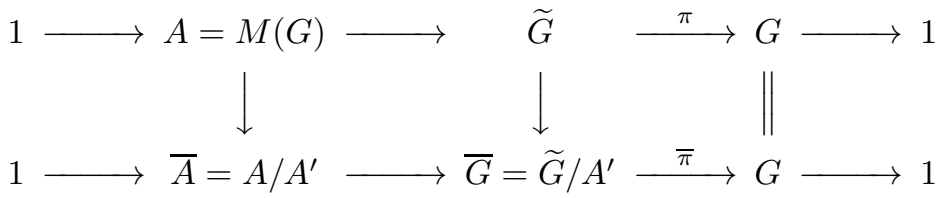

with $\bar{A} \simeq \mathbb{Z} / 2 \mathbb{Z}$ via the function MinimalStemExtensions $(G)[j]$.MinimalStemExtension. Then we apply Theorem 6.5 instead of Theorem 6.6.

(1-4-1) The case $G=8 T 31 \simeq\left(\left(C_{2}\right)^{4} \rtimes C_{2}\right) \rtimes C_{2}$. We have $H \simeq\left(C_{2}\right)^{3}$ and $M(G) \simeq(\mathbb{Z} / 2 \mathbb{Z})^{\oplus 4}$. Applying the function MinimalStemExtensions $(G)[j]$.MinimalStemExtension, we get the minimal stem ex-

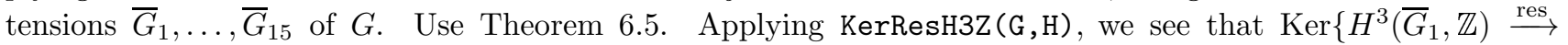
$\left.\oplus_{i=1}^{m^{\prime}} H^{3}\left(G_{i}, \mathbb{Z}\right)\right\}=0$ but $\operatorname{Ker}\left\{H^{3}\left(\bar{G}_{j}, \mathbb{Z}\right) \stackrel{\text { res }}{\longrightarrow} \oplus_{i=1}^{m^{\prime}} H^{3}\left(G_{i}, \mathbb{Z}\right)\right\} \simeq \mathbb{Z} / 2 \mathbb{Z}$ for $j \in J:=\{j \mid 2 \leq j \leq 15\}$. Because $\oplus_{i=1}^{m^{\prime}} \widehat{H}^{-3}\left(G_{i}, \mathbb{Z}\right) \stackrel{\text { cores }}{\longrightarrow} \widehat{H}^{-3}\left(\bar{G}_{1}, \mathbb{Z}\right)$ is surjective, it follows from Theorem 6.5 that $\operatorname{Obs}(K / k)=\operatorname{Obs}_{1}\left(\bar{L}_{1} / K / k\right)$. We also checked that $\operatorname{Ker} \bar{\psi}_{1} / \bar{\varphi}_{1}\left(\operatorname{Ker} \bar{\psi}_{1}^{\mathrm{nr}}\right) \simeq \mathbb{Z} / 2 \mathbb{Z}$ for $\bar{G}_{1}$ and $\operatorname{Ker} \bar{\psi}_{1} / \bar{\varphi}_{1}\left(\operatorname{Ker} \bar{\psi}_{2}^{\mathrm{nr}}\right)=0$ for $\bar{G}_{j}(j \in J)$. This implies that $\operatorname{Obs}(K / k) \neq \operatorname{Obs}_{1}\left(\bar{L}_{j} / K / k\right)$ when $\bar{L}_{j} / k$ is unramified for $j \in J$. Apply FirstObstructionDr $\left(\bar{G}_{1}, \bar{G}_{1}^{\prime}, \bar{H}_{1}\right)$ for all subgroups $\bar{G}_{1}^{\prime} \leq \bar{G}_{1}$. We find that $\operatorname{Obs}_{1}\left(\bar{L}_{1} / K / k\right)=1$ if and only if there exists $v \in V_{k}$ such that (i) $V_{4} \leq G_{v}$ where $V_{4} \cap[G, G]=1$ (equivalently, $\left|\operatorname{Orb}_{V_{4}}(i)\right|=4$ for any $1 \leq i \leq 8$ and $V_{4} \cap Z(G)=1$ ), (ii) $C_{4} \times C_{2} \leq G_{v}$ where $\left(C_{4} \times C_{2}\right) \cap[G, G] \simeq C_{2}$ (equivalently, $C_{4} \times C_{2}$ is transitive in $\left.S_{8}\right)$ or (iii) $\left(C_{2}\right)^{3} \rtimes C_{4} \leq G_{v}$ (see Details 6.8 and Example 6.11).

(1-4-2) The case $G=8 T 38 \simeq\left(\left(\left(C_{2}\right)^{4} \rtimes C_{2}\right) \rtimes C_{2}\right) \rtimes C_{3}$. We have $H \simeq C_{2} \times A_{4}$ and $M(G) \simeq(\mathbb{Z} / 2 \mathbb{Z})^{\oplus 2}$. Applying the function MinimalStemExtensions $(G)[j]$.MinimalStemExtension, we get the minimal stem ex-

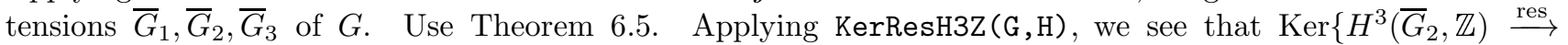
$\left.\oplus_{i=1}^{m^{\prime}} H^{3}\left(G_{i}, \mathbb{Z}\right)\right\}=0$ but $\operatorname{Ker}\left\{H^{3}\left(\bar{G}_{j}, \mathbb{Z}\right) \stackrel{\text { res }}{\longrightarrow} \oplus_{i=1}^{m^{\prime}} H^{3}\left(G_{i}, \mathbb{Z}\right)\right\} \simeq \mathbb{Z} / 2 \mathbb{Z}$ for $j \in J:=\{1,3\}$. We have that $\oplus_{i=1}^{m^{\prime}} \widehat{H}^{-3}\left(G_{i}, \mathbb{Z}\right) \stackrel{\text { cores }}{\longrightarrow} \widehat{H}^{-3}\left(\bar{G}_{2}, \mathbb{Z}\right)$ is surjective. By Theorem 6.5. $\operatorname{Obs}(K / k)=\operatorname{Obs}_{1}\left(\bar{L}_{2} / K / k\right)$. We also checked that $\operatorname{Ker} \bar{\psi}_{1} / \bar{\varphi}_{1}\left(\operatorname{Ker} \bar{\psi}_{1}^{\mathrm{nr}}\right) \simeq \mathbb{Z} / 2 \mathbb{Z}$ for $\bar{G}_{2}$ and $\operatorname{Ker} \bar{\psi}_{1} / \bar{\varphi}_{1}\left(\operatorname{Ker} \bar{\psi}_{2}^{\mathrm{nr}}\right)=0$ for $\bar{G}_{j}(j \in J)$. This implies that $\operatorname{Obs}(K / k) \neq \operatorname{Obs}_{1}\left(\bar{L}_{j} / K / k\right)$ when $\bar{L}_{j} / k$ is unramified for $j \in J$. Apply FirstObstructionDr $\left(\bar{G}_{2}, \bar{G}_{2}^{\prime}, \bar{H}_{2}\right)$ for all subgroups $\bar{G}_{2}^{\prime} \leq \bar{G}_{2}$. We find that $\operatorname{Obs}_{1}\left(\bar{L}_{2} / K / k\right)=1$ if and only if there exists $v \in V_{k}$ such that (i) $V_{4} \leq G_{v}$ where $V_{4} \cap\left[\operatorname{Syl}_{2}(G), \operatorname{Syl}_{2}(G)\right]=1$ with $\operatorname{Syl}_{2}(G) \triangleleft G$ (equivalently, $\left|\operatorname{Orb}_{V_{4}}(i)\right|=4$ for any $1 \leq i \leq 8$ and $V_{4} \cap Z(G)=1$ ), (ii) $C_{4} \times C_{2} \leq G_{v}$ where $\left(C_{4} \times C_{2}\right) \cap\left[\operatorname{Syl}_{2}(G)\right.$, $\left.\operatorname{Syl}_{2}(G)\right] \simeq C_{2}$ (equivalently, $C_{4} \times C_{2}$ is transitive in $S_{8}$ ) or (iii) $\left(C_{2}\right)^{3} \rtimes C_{4} \leq G_{v}$ (see Details 6.8 and Example 6.11).

(2) $n=8: G=8 \mathrm{Tm}(m=9,11,15,19,22,32)$. We assume that $H=\operatorname{Stab}_{1}(G)$ by the assumption. Applying FirstObstructionN $(G)$, we have $\operatorname{Obs}_{1}(L / K / k)=1$. For the cases $G=8 T m(m=9,11,15,19,22,32)$, we also find that $(\mathbb{Z} / 2 \mathbb{Z})^{\oplus 2} \leq M(G) \leq(\mathbb{Z} / 2 \mathbb{Z})^{\oplus 5}$. Hence we take a minimal stem extension $\bar{G}=\widetilde{G} / A^{\prime}$, i.e. $\bar{A} \leq Z(\bar{G}) \cap[\bar{G}, \bar{G}]$, of $G$ in the commutative diagram

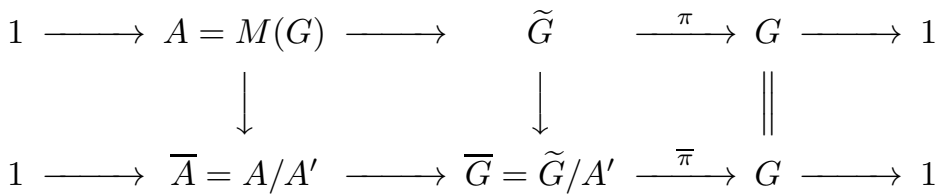

with $\bar{A} \simeq \mathbb{Z} / 2 \mathbb{Z}$ via the function MinimalStemExtensions $(G)[j]$.MinimalStemExtension. Then we apply Theorem 6.5 instead of Theorem 6.6 as in the case (1-4).

(2-1) The case $G=8 T 9 \simeq D_{4} \times C_{2}$. We have $H \simeq C_{2}$. We obtain that the Schur multiplier $M(G) \simeq$ $(\mathbb{Z} / 2 \mathbb{Z})^{\oplus 3}$. By applying MinimalStemExtensions $(G)[j]$. MinimalStemExtension, we obtain the minimal stem

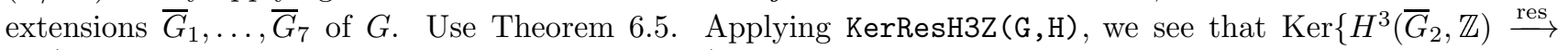
$\left.\oplus_{i=1}^{m^{\prime}} H^{3}\left(G_{i}, \mathbb{Z}\right)\right\}=0$ but $\operatorname{Ker}\left\{H^{3}\left(\bar{G}_{j}, \mathbb{Z}\right) \stackrel{\text { res }}{\longrightarrow} \oplus_{i=1}^{m^{\prime}} H^{3}\left(G_{i}, \mathbb{Z}\right)\right\}=\mathbb{Z} / 2 \mathbb{Z}$ for $j \in J:=\{1,3,4,5,6,7\}$. Because 
$\oplus_{i=1}^{m^{\prime}} \widehat{H}^{-3}\left(G_{i}, \mathbb{Z}\right) \stackrel{\text { cores }}{\longrightarrow} \widehat{H}^{-3}\left(\bar{G}_{2}, \mathbb{Z}\right)$ is surjective, it follows from Theorem 6.5 that $\operatorname{Obs}(K / k)=\operatorname{Obs}_{1}\left(\bar{L}_{2} / K / k\right)$. We also checked that $\operatorname{Ker} \bar{\psi}_{1} / \bar{\varphi}_{1}\left(\operatorname{Ker} \bar{\psi}_{2}^{\mathrm{nr}}\right) \simeq \mathbb{Z} / 2 \mathbb{Z}$ for $\bar{G}_{2}$ and $\operatorname{Ker} \bar{\psi}_{1} / \bar{\varphi}_{1}\left(\operatorname{Ker} \bar{\psi}_{2}^{\mathrm{nr}}\right)=0$ for $\bar{G}_{j}(j \in J)$. Hence $\operatorname{Obs}(K / k) \neq \operatorname{Obs}_{1}\left(\bar{L}_{j} / K / k\right)$ when $\bar{L}_{j} / k$ is unramified for $j \in J$. Apply FirstObstructionDr $\left(\bar{G}_{2}, \bar{G}_{2}^{\prime}, \bar{H}_{2}\right)$ for all subgroups $\bar{G}_{2}^{\prime} \leq \bar{G}_{2}$. We find that $\operatorname{Obs}_{1}\left(\bar{L}_{2} / K / k\right)=1$ if and only if there exists $v \in V_{k}$ such that (i) $V_{4} \leq G_{v}$ where $\left|\operatorname{Orb}_{V_{4}}(i)\right|=4$ for any $1 \leq i \leq 8$ and $V_{4} \cap[G, G]=1$; or (ii) $C_{4} \times C_{2} \leq G_{v}$ (see Details 6.8 and Example 6.12).

(2-2) The case $G=8 T 11 \simeq\left(C_{4} \times C_{2}\right) \rtimes C_{2}$. We have $H \simeq C_{2}$ and $M(G) \simeq(\mathbb{Z} / 2 \mathbb{Z})^{\oplus 3}$. Applying MinimalStemExtensions $(G)[j]$.MinimalStemExtension. We get the minimal stem extensions $\bar{G}_{1}, \bar{G}_{2}, \bar{G}_{3}$ of

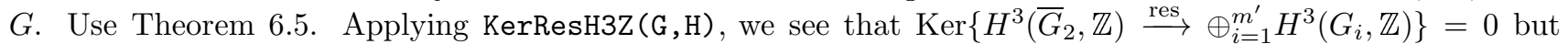
$\operatorname{Ker}\left\{H^{3}\left(\bar{G}_{j}, \mathbb{Z}\right) \stackrel{\text { res }}{\longrightarrow} \oplus_{i=1}^{m^{\prime}} H^{3}\left(G_{i}, \mathbb{Z}\right)\right\}=\mathbb{Z} / 2 \mathbb{Z}$ for $j \in J:=\{1,3\}$. Because $\oplus_{i=1}^{m^{\prime}} \widehat{H}^{-3}\left(G_{i}, \mathbb{Z}\right) \stackrel{\text { cores }}{\longrightarrow} \widehat{H}^{-3}\left(\bar{G}_{2}, \mathbb{Z}\right)$ is surjective, it follows from Theorem6.5 that $\operatorname{Obs}(K / k)=\operatorname{Obs}_{1}\left(\bar{L}_{2} / K / k\right)$. We also checked that $\operatorname{Ker} \bar{\psi}_{1} / \bar{\varphi}_{1}\left(\operatorname{Ker} \bar{\psi}_{2}^{\mathrm{nr}}\right)$ $\simeq \mathbb{Z} / 2 \mathbb{Z}$ for $\bar{G}_{2}$ and $\operatorname{Ker} \bar{\psi}_{1} / \bar{\varphi}_{1}\left(\operatorname{Ker} \bar{\psi}_{2}^{\mathrm{nr}}\right)=0$ for $\bar{G}_{j}(j \in J)$. This implies that $\operatorname{Obs}(K / k) \neq \operatorname{Obs}_{1}\left(\bar{L}_{j} / K / k\right)$ when $\bar{L}_{j} / k$ is unramified for $j \in J$. Apply FirstObstructionDr $\left(\bar{G}_{2}, \bar{G}_{2}^{\prime}, \bar{H}_{2}\right)$ for all subgroups $\bar{G}_{2}^{\prime} \leq \bar{G}_{2}$. We find that $\operatorname{Obs}_{1}\left(\bar{L}_{2} / K / k\right)=1$ if and only if there exists $v \in V_{k}$ such that $C_{4} \times C_{2} \leq G_{v}$ where $C_{4} \times C_{2}$ is transitive in $S_{8}$ (see Details 6.8 and Example 6.12).

(2-3) The case $G=8 T 15 \simeq C_{8} \rtimes V_{4}$. We have $H \simeq V_{4}$ and $M(G) \simeq(\mathbb{Z} / 2 \mathbb{Z})^{\oplus 2}$. Applying the function MinimalStemExtensions $(G)[j]$.MinimalStemExtension, we get the minimal stem extensions $\bar{G}_{1}, \bar{G}_{2}, \bar{G}_{3}$

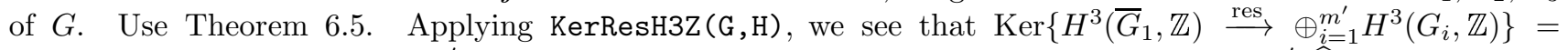
0 but $\operatorname{Ker}\left\{H^{3}\left(\bar{G}_{j}, \mathbb{Z}\right) \stackrel{\text { res }}{\longrightarrow} \oplus_{i=1}^{m^{\prime}} H^{3}\left(G_{i}, \mathbb{Z}\right)\right\}=\mathbb{Z} / 2 \mathbb{Z}$ for $j \in J:=\{2,3\}$. Because $\oplus_{i=1}^{m^{\prime}} \hat{H}^{-3}\left(G_{i}, \mathbb{Z}\right) \stackrel{\text { cores }}{\longrightarrow}$ $\widehat{H}^{-3}\left(\bar{G}_{1}, \mathbb{Z}\right)$ is surjective, it follows from Theorem 6.5 that $\operatorname{Obs}(K / k)=\operatorname{Obs}_{1}\left(\bar{L}_{1} / K / k\right)$. We also checked that $\operatorname{Ker} \bar{\psi}_{1} / \bar{\varphi}_{1}\left(\operatorname{Ker} \bar{\psi}_{1}^{\mathrm{nr}}\right) \simeq \mathbb{Z} / 2 \mathbb{Z}$ for $\bar{G}_{1}$ and $\operatorname{Ker} \bar{\psi}_{1} / \bar{\varphi}_{1}\left(\operatorname{Ker} \bar{\psi}_{2}^{\mathrm{nr}}\right)=0$ for $\bar{G}_{j}(j \in J)$. This implies that $\operatorname{Obs}(K / k) \neq \operatorname{Obs}_{1}\left(\bar{L}_{j} / K / k\right)$ when $\bar{L}_{j} / k$ is unramified for $j \in J$. Apply FirstObstructionDr $\left(\bar{G}_{1}, \bar{G}_{1}^{\prime}, \bar{H}_{1}\right)$ for all subgroups $\bar{G}_{1}^{\prime} \leq \bar{G}_{1}$. We find that $\operatorname{Obs}_{1}\left(\bar{L}_{1} / K / k\right)=1$ if and only if there exists $v \in V_{k}$ such that (i) $V_{4} \leq G_{v}$ where $\left|\operatorname{Orb}_{V_{4}}(i)\right|=2$ for any $1 \leq i \leq 8$ and $V_{4} \cap[G, G]=1$ (equivalently, $\left|\operatorname{Orb}_{V_{4}}(i)\right|=2$ for any $1 \leq i \leq 8$ and $V_{4}$ is not in $\left.A_{8}\right)$ or (ii) $C_{4} \times C_{2} \leq G_{v}$ where $\left(C_{4} \times C_{2}\right) \cap[G, G] \simeq C_{2}$ (equivalently, $C_{4} \times C_{2}$ is transitive in $S_{8}$ ) (see Details 6.8 and Example 6.12 ).

(2-4) The case $G=8 T 19 \simeq\left(C_{2}\right)^{3} \rtimes C_{4}$. We have $H \simeq C_{4}$ and $M(G) \simeq(\mathbb{Z} / 2 \mathbb{Z})^{\oplus 2}$. Applying the function MinimalStemExtensions $(G)[j]$.MinimalStemExtension, we get the minimal stem extensions $\bar{G}_{1}, \bar{G}_{2}, \bar{G}_{3}$

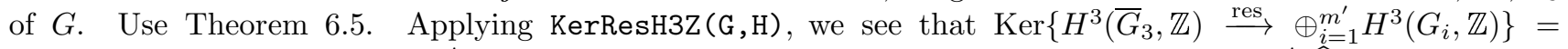
0 but $\operatorname{Ker}\left\{H^{3}\left(\bar{G}_{j}, \mathbb{Z}\right) \stackrel{\text { res }}{\longrightarrow} \oplus_{i=1}^{m^{\prime}} H^{3}\left(G_{i}, \mathbb{Z}\right)\right\}=\mathbb{Z} / 2 \mathbb{Z}$ for $j \in J:=\{1,2\}$. Because $\oplus_{i=1}^{m^{\prime}} \hat{H}^{-3}\left(G_{i}, \mathbb{Z}\right) \stackrel{\text { cores }}{\longrightarrow}$ $\widehat{H}^{-3}\left(\bar{G}_{3}, \mathbb{Z}\right)$ is surjective, it follows from Theorem 6.5 that $\operatorname{Obs}(K / k)=\mathrm{Obs}_{1}\left(\bar{L}_{3} / K / k\right)$. We also checked that $\operatorname{Ker} \bar{\psi}_{1} / \bar{\varphi}_{1}\left(\operatorname{Ker} \bar{\psi}_{1}^{\mathrm{nr}}\right) \simeq \mathbb{Z} / 2 \mathbb{Z}$ for $\bar{G}_{3}$ and $\operatorname{Ker} \bar{\psi}_{1} / \bar{\varphi}_{1}\left(\operatorname{Ker} \bar{\psi}_{2}^{\mathrm{nr}}\right)=0$ for $\bar{G}_{j}(j \in J)$. This implies that $\operatorname{Obs}(K / k) \neq \operatorname{Obs}_{1}\left(\bar{L}_{j} / K / k\right)$ when $\bar{L}_{j} / k$ is unramified for $j \in J$. Apply FirstObstructionDr $\left(\bar{G}_{3}, \bar{G}_{3}^{\prime}, \bar{H}_{3}\right)$ for all subgroups $\bar{G}_{3}^{\prime} \leq \bar{G}_{3}$. We find that $\operatorname{Obs}_{1}\left(\bar{L}_{3} / K / k\right)=1$ if and only if there exists $v \in V_{k}$ such that (i) $V_{4} \leq G_{v}$ where $V_{4} \cap Z(G)=1$ and $V_{4} \cap Z^{2}(G) \simeq C_{2}$ with the upper central series $1 \leq Z(G) \leq Z^{2}(G) \leq G$ of $G$ (equivalently, $\left|\operatorname{Orb}_{V_{4}}(i)\right|=4$ for any $1 \leq i \leq 8$ and $V_{4} \cap Z(G)=1$ ); or (ii) $C_{4} \times C_{2} \leq G_{v}$ where $C_{4} \times C_{2}$ is not transitive in $S_{8}$ or $[G, G] \leq C_{4} \times C_{2}$ (see Details 6.8 and Example 6.12).

$(2-5)$ The case $G=8 T 22 \simeq\left(C_{2}\right)^{3} \rtimes V_{4}$. We have $H \simeq V_{4}$ and $M(G) \simeq(\mathbb{Z} / 2 \mathbb{Z})^{\oplus 5}$. Applying the function MinimalStemExtensions $(G)[j]$.MinimalStemExtension, we get the minimal stem extensions $\bar{G}_{1}, \ldots, \bar{G}_{31}$ of

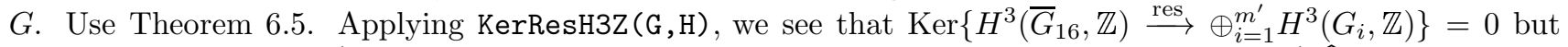
$\operatorname{Ker}\left\{H^{3}\left(\bar{G}_{j}, \mathbb{Z}\right) \stackrel{\text { res }}{\longrightarrow} \oplus_{i=1}^{m^{\prime}} H^{3}\left(G_{i}, \mathbb{Z}\right)\right\} \neq 0$ for $j \in J:=\{j \mid 1 \leq j \leq 31, j \neq 16\}$. Because $\oplus_{i=1}^{m^{\prime}} \widehat{H}^{-3}\left(G_{i}, \mathbb{Z}\right) \stackrel{\text { cores }}{\longrightarrow}$ $\widehat{H}^{-3}\left(\bar{G}_{16}, \mathbb{Z}\right)$ is surjective, it follows from Theorem 6.5 that $\operatorname{Obs}(K / k)=\mathrm{Obs}_{1}\left(\bar{L}_{16} / K / k\right)$. We also checked that $\operatorname{Ker} \bar{\psi}_{1} / \bar{\varphi}_{1}\left(\operatorname{Ker} \bar{\psi}_{1}^{\mathrm{nr}}\right) \simeq \mathbb{Z} / 2 \mathbb{Z}$ for $\bar{G}_{16}$ and $\operatorname{Ker} \bar{\psi}_{1} / \bar{\varphi}_{1}\left(\operatorname{Ker} \bar{\psi}_{2}^{\mathrm{nr}}\right)=0$ for $\bar{G}_{j}(j \in J)$. This implies that $\operatorname{Obs}(K / k) \neq \operatorname{Obs}_{1}\left(\bar{L}_{j} / K / k\right)$ when $\bar{L}_{j} / k$ is unramified for $j \in J$. Apply FirstObstructionDr $\left(\bar{G}_{16}, \bar{G}_{16}^{\prime}, \bar{H}_{16}\right)$ for all subgroups $\bar{G}_{16}^{\prime} \leq \bar{G}_{16}$. We find that $\operatorname{Obs}_{1}\left(\bar{L}_{16} / K / k\right)=1$ if and only if there exists $v \in V_{k}$ such that (i) $V_{4} \leq G_{v}$ where $\left|\operatorname{Orb}_{V_{4}}(i)\right|=4$ for any $1 \leq i \leq 8$ and $V_{4} \cap Z(G)=1$ or (ii) $C_{4} \times C_{2} \leq G_{v}$ where $C_{4} \times C_{2}$ is transitive in $S_{8}$. (see Details 6.8 and Example 6.12).

(2-6) The case $G=8 T 32 \simeq\left(\left(C_{2}\right)^{3} \rtimes V_{4}\right) \rtimes C_{3}$. We have $H \simeq A_{4}$ and $M(G) \simeq(\mathbb{Z} / 2 \mathbb{Z})^{\oplus 3}$. Applying the function MinimalStemExtensions $(G)[j]$.MinimalStemExtension, we get the minimal stem extensions $\bar{G}_{1}, \ldots, \bar{G}_{7}$ of

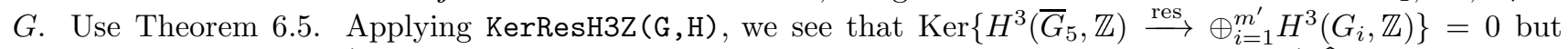
$\operatorname{Ker}\left\{H^{3}\left(\bar{G}_{j}, \mathbb{Z}\right) \stackrel{\text { res }}{\longrightarrow} \oplus_{i=1}^{m^{\prime}} H^{3}\left(G_{i}, \mathbb{Z}\right)\right\} \simeq \mathbb{Z} / 2 \mathbb{Z}$ for $j \in J:=\{j \mid 2 \leq j \leq 7\}$. Because $\oplus_{i=1}^{m^{\prime}} \widehat{H}^{-3}\left(G_{i}, \mathbb{Z}\right) \stackrel{\text { cores }}{\longrightarrow}$ $\widehat{H}^{-3}\left(\bar{G}_{5}, \mathbb{Z}\right)$ is surjective, it follows from Theorem 6.5 that $\operatorname{Obs}(K / k)=\operatorname{Obs}_{1}\left(\bar{L}_{5} / K / k\right)$. We also checked that $\operatorname{Ker} \bar{\psi}_{1} / \bar{\varphi}_{1}\left(\operatorname{Ker} \bar{\psi}_{1}^{\mathrm{nr}}\right) \simeq \mathbb{Z} / 2 \mathbb{Z}$ for $\bar{G}_{5}$ and $\operatorname{Ker} \bar{\psi}_{1} / \bar{\varphi}_{1}\left(\operatorname{Ker} \bar{\psi}_{2}^{\mathrm{nr}}\right)=0$ for $\bar{G}_{j}(j \in J)$. This implies that $\operatorname{Obs}(K / k) \neq$ $\operatorname{Obs}_{1}\left(\bar{L}_{j} / K / k\right)$ when $\bar{L}_{j} / k$ is unramified for $j \in J$. Apply FirstobstructionDr $\left(\bar{G}_{1}, \bar{G}_{1}^{\prime}, \bar{H}_{1}\right)$ for all subgroups $\bar{G}_{1}^{\prime} \leq \bar{G}_{1}$. We find that $\operatorname{Obs}_{1}\left(\bar{L}_{1} / K / k\right)=1$ if and only if there exists $v \in V_{k}$ such that (i) $V_{4} \leq G_{v}$ where 
$\left|\operatorname{Orb}_{V_{4}}(i)\right|=4$ for any $1 \leq i \leq 8$ and $V_{4} \cap Z(G)=1$ or (ii) $C_{4} \times C_{2} \leq G_{v}$ where $C_{4} \times C_{2}$ is transitive in $S_{8}$ (see Details 6.8 and Example 6.12).

(3) $n=9: G=9 \mathrm{Tm}(m=2,5,7,9,11,14,23)$. Applying FirstObstructionN $(G)$, we have $\operatorname{Obs}_{1}(L / K / k)=$ 1 for each cases. We apply Theorem 6.6 for $m=2,5,9,11,14,23$. We see that $M(G) \simeq \mathbb{Z} / 3 \mathbb{Z}$ for $m=$ $2,5,9,11,14,23$ and $M(G) \simeq(\mathbb{Z} / 3 \mathbb{Z})^{\oplus 2}$ for $m=7$. Then for $m=7$ we apply Theorem 6.5 instead of Theorem 6.6 as in the case $(2) n=8$.

(3-1) The case $G=9 T 2 \simeq\left(C_{3}\right)^{2}$ (see also Theorem 1.8 because $H=1$ ). Apply Theorem6.6. We obtain a Schur cover $1 \rightarrow M(G) \simeq \mathbb{Z} / 3 \mathbb{Z} \rightarrow \widetilde{G} \stackrel{\pi}{\rightarrow} G \rightarrow 1$ with $\widetilde{G} \simeq\left(C_{3}\right)^{2} \rtimes C_{3}$ and $\widetilde{H} \simeq C_{3}$ and $\operatorname{Obs}(K / k)=\operatorname{Obs}_{1}(\widetilde{L} / K / k)$. By Theorem 1.15, Ker $\widetilde{\psi}_{1} / \widetilde{\varphi}_{1}\left(\operatorname{Ker} \widetilde{\psi}_{2}^{\mathrm{nr}}\right) \simeq \mathbb{Z} / 3 \mathbb{Z}$. By applying First0bstructionDr $\left(\widetilde{G}, \widetilde{G}^{\prime}, \widetilde{H}\right)$ for all subgroups $\widetilde{G}^{\prime} \leq \widetilde{G}$, we obtain that $\operatorname{Obs}_{1}(\widetilde{L} / K / k)=1$ if and only if there exists $v \in V_{k}$ such that $\widetilde{G}_{v}=\widetilde{G}$ if and only if there exists $v \in V_{k}$ such that $G_{v}=G$ (see Example 6.13).

(3-2) The case $G=9 T 5 \simeq\left(C_{3}\right)^{2} \rtimes C_{2}$. We have $H \simeq C_{2}$. Apply Theorem 6.6 as in the case (2) $n=8$. We obtain a Schur cover $1 \rightarrow M(G) \simeq \mathbb{Z} / 3 \mathbb{Z} \rightarrow \widetilde{G} \stackrel{\pi}{\rightarrow} G \rightarrow 1$ with $\widetilde{G} \simeq\left(\left(C_{3}\right)^{2} \rtimes C_{3}\right) \rtimes C_{2}$ and $\widetilde{H} \simeq C_{6}$ and Obs $(K / k)=$ $\operatorname{Obs}_{1}(\widetilde{L} / K / k)$. By Theorem 1.15, Ker $\widetilde{\psi}_{1} / \widetilde{\varphi}_{1}\left(\operatorname{Ker} \widetilde{\psi}_{2}^{\mathrm{nr}}\right) \simeq \mathbb{Z} / 3 \mathbb{Z}$. By applying FirstObstructionDr $\left(\widetilde{G}, \widetilde{G}^{\prime}, \widetilde{H}\right)$ for all subgroups $\widetilde{G}^{\prime} \leq \widetilde{G}$, we obtain that $\operatorname{Obs}_{1}(\widetilde{L} / K / k)=1$ if and only if there exists $v \in V_{k}$ such that $\left(C_{3}\right)^{2} \rtimes C_{3} \leq \widetilde{G}_{v}$ if and only if there exists $v \in V_{k}$ such that $\left(C_{3}\right)^{2} \leq G_{v}$ (see Example 6.13).

(3-3) The case $G=9 T 7 \simeq\left(C_{3}\right)^{2} \rtimes C_{3}$. We have $H \simeq C_{3}$ and $M(G) \simeq(\mathbb{Z} / 3 \mathbb{Z})^{\oplus 2}$. Applying the function MinimalStemExtensions $(G)[j]$. MinimalStemExtension, we get the minimal stem extensions $\bar{G}_{1}, \ldots, \bar{G}_{4}$ of $G$. Use Theorem 6.5. Applying $\operatorname{KerResH3Z}(\mathrm{G}, \mathrm{H})$, we obtain that $\operatorname{Ker}\left\{H^{3}\left(\bar{G}_{1}, \mathbb{Z}\right) \stackrel{\text { res }}{\longrightarrow} \oplus_{i=1}^{m^{\prime}} H^{3}\left(G_{i}, \mathbb{Z}\right)\right\}=0$ but $\operatorname{Ker}\left\{H^{3}\left(\bar{G}_{j}, \mathbb{Z}\right) \stackrel{\text { res }}{\longrightarrow} \oplus_{i=1}^{m^{\prime}} H^{3}\left(G_{i}, \mathbb{Z}\right)\right\} \simeq \mathbb{Z} / 3 \mathbb{Z}$ for $j \in J:=\{2,3,4\}$. Because $\oplus_{i=1}^{m^{\prime}} \widehat{H}^{-3}\left(G_{i}, \mathbb{Z}\right) \stackrel{\text { cores }}{\longrightarrow} \widehat{H}^{-3}\left(\bar{G}_{1}, \mathbb{Z}\right)$ is surjective, it follows from Theorem 6.5 that $\operatorname{Obs}(K / k)=\operatorname{Obs}_{1}\left(\bar{L}_{1} / K / k\right)$. We also have $\operatorname{Ker} \bar{\psi}_{1} / \bar{\varphi}_{1}\left(\operatorname{Ker} \bar{\psi}_{1}^{\text {nr }}\right) \simeq$ $\mathbb{Z} / 2 \mathbb{Z}$ for $\bar{G}_{1}$ and $\operatorname{Ker} \bar{\psi}_{1} / \bar{\varphi}_{1}\left(\operatorname{Ker} \bar{\psi}_{2}^{\mathrm{nr}}\right)=0$ for $\bar{G}_{j}(j \in J)$. This implies that $\operatorname{Obs}(K / k) \neq \operatorname{Obs}_{1}\left(\bar{L}_{j} / K / k\right)$ when $\bar{L}_{j} / k$ is unramified for $j \in J$. Apply FirstObstructionDr $\left(\bar{G}_{1}, \bar{G}_{1}^{\prime}, \bar{H}_{1}\right)$ for all subgroups $\bar{G}_{1}^{\prime} \leq \bar{G}_{1}$. We find that $\operatorname{Obs}_{1}\left(\bar{L}_{1} / K / k\right)=1$ if and only if there exists $v \in V_{k}$ such that $\left(C_{3}\right)^{2} \leq \bar{G}_{v}$ if and only if there exists $v \in V_{k}$ such that $\left(C_{3}\right)^{2} \leq G_{v}$ (see Example 6.13).

(3-4) The case $G=9 T 9 \simeq\left(C_{3}\right)^{2} \rtimes C_{4}$. We have $H \simeq C_{4}$. Apply Theorem 6.6. We obtain a Schur cover $1 \rightarrow M(G) \simeq \mathbb{Z} / 3 \mathbb{Z} \rightarrow \widetilde{G} \stackrel{\pi}{\rightarrow} G \rightarrow 1$ with $\widetilde{G} \simeq\left(\left(C_{3}\right)^{2} \rtimes C_{3}\right) \rtimes C_{4}, \widetilde{H} \simeq C_{12}$ and $\operatorname{Obs}(K / k)=\operatorname{Obs}_{1}(\widetilde{L} / K / k)$. By Theorem 1.15. Ker $\widetilde{\psi}_{1} / \widetilde{\varphi}_{1}\left(\operatorname{Ker} \widetilde{\psi}_{2}^{\text {nr }}\right) \simeq \mathbb{Z} / 3 \mathbb{Z}$. By applying FirstObstructionDr $\left(\widetilde{G}, \widetilde{G}^{\prime}, \widetilde{H}\right)$ for all subgroups $\widetilde{G}^{\prime} \leq \widetilde{G}$, we obtain that $\operatorname{Obs}_{1}(\widetilde{L} / K / k)=1$ if and only if there exists $v \in V_{k}$ such that $\left(C_{3}\right)^{2} \rtimes C_{3} \leq \widetilde{G}_{v}$ if and only if there exists $v \in V_{k}$ such that $\left(C_{3}\right)^{2} \leq G_{v}$ (see Example 6.13).

(3-5) The case $G=9 T 11 \simeq\left(C_{3}\right)^{2} \rtimes C_{6}$. We have $H \simeq C_{6}$. Apply Theorem 6.6. We obtain a Schur cover $1 \rightarrow M(G) \simeq \mathbb{Z} / 3 \mathbb{Z} \rightarrow \widetilde{G} \stackrel{\pi}{\rightarrow} G \rightarrow 1$ with $\widetilde{G} \simeq\left(\left(C_{3}\right)^{3} \rtimes C_{3}\right) \rtimes C_{2}, \widetilde{H} \simeq C_{6} \times C_{3}$ and $\operatorname{Obs}(K / k)=\operatorname{Obs}_{1}(\widetilde{L} / K / k)$. By Theorem 1.15, Ker $\widetilde{\psi}_{1} / \widetilde{\varphi}_{1}\left(\operatorname{Ker} \widetilde{\psi}_{2}^{\text {nr }}\right) \simeq \mathbb{Z} / 3 \mathbb{Z}$. By applying FirstobstructionDr $\left(\widetilde{G}, \widetilde{G}^{\prime}, \widetilde{H}\right)$ for all subgroups $\widetilde{G}^{\prime} \leq \widetilde{G}$, we obtain that $\operatorname{Obs}_{1}(\widetilde{L} / K / k)=1$ if and only if there exists $v \in V_{k}$ such that $\left(C_{3}\right)^{2} \leq G_{v}$ (see Example $6.13)$.

(3-6) The case $G=9 T 14 \simeq\left(C_{3}\right)^{2} \rtimes Q_{8}$. We have $H \simeq Q_{8}$. Apply Theorem 6.6. We obtain a Schur cover $1 \rightarrow M(G) \simeq \mathbb{Z} / 3 \mathbb{Z} \rightarrow \widetilde{G} \stackrel{\pi}{\rightarrow} G \rightarrow 1$ with $\widetilde{G} \simeq\left(\left(\left(C_{3}\right)^{2} \rtimes C_{3}\right) \rtimes Q_{8}\right) \rtimes C_{3}, \widetilde{H} \simeq C_{3} \times \mathrm{SL}_{2}\left(\mathbb{F}_{3}\right)$ and $\operatorname{Obs}(K / k)=$ $\operatorname{Obs}_{1}(\widetilde{L} / K / k)$. By Theorem 1.15, Ker $\widetilde{\psi}_{1} / \widetilde{\varphi}_{1}\left(\operatorname{Ker} \widetilde{\psi}_{2}^{\mathrm{nr}}\right) \simeq \mathbb{Z} / 3 \mathbb{Z}$. By applying FirstObstructionDr $\left(\widetilde{G}, \widetilde{G}^{\prime}, \widetilde{H}\right)$ for all subgroups $\widetilde{G}^{\prime} \leq \widetilde{G}$, we obtain that $\operatorname{Obs}_{1}(\widetilde{L} / K / k)=1$ if and only if there exists $v \in V_{k}$ such that $\left(C_{3}\right)^{2} \rtimes C_{3} \leq \widetilde{G}_{v}$ if and only if there exists $v \in V_{k}$ such that $\left(C_{3}\right)^{2} \leq G_{v}$ (see Example 6.13).

(3-7) The case $G=9 T 23 \simeq\left(\left(C_{3}\right)^{2} \rtimes Q_{8}\right) \rtimes C_{3}$. We have $H \simeq \mathrm{SL}_{2}\left(\mathbb{F}_{3}\right)$. Apply Theorem 6.6 We obtain a Schur cover $1 \rightarrow M(G) \simeq \mathbb{Z} / 3 \mathbb{Z} \rightarrow \widetilde{G} \stackrel{\pi}{\rightarrow} G \rightarrow 1$ with $\widetilde{G} \simeq\left(\left(\left(C_{3}\right)^{2} \rtimes C_{3}\right) \rtimes Q_{8}\right) \rtimes C_{3}, \widetilde{H} \simeq C_{3} \times \mathrm{SL}_{2}\left(\mathbb{F}_{3}\right)$ and Obs $(K / k)=$ $\operatorname{Obs}_{1}(\widetilde{L} / K / k)$. By Theorem 1.15] $\operatorname{Ker} \widetilde{\psi}_{1} / \widetilde{\varphi}_{1}\left(\operatorname{Ker} \widetilde{\psi}_{2}^{\text {nr }}\right) \simeq \mathbb{Z} / 3 \mathbb{Z}$. By applying FirstObstructionDr $\left(\widetilde{G}, \widetilde{G}^{\prime}, \widetilde{H}\right)$ for all subgroups $\widetilde{G}^{\prime} \leq \widetilde{G}$, we obtain that $\operatorname{Obs}_{1}(\widetilde{L} / K / k)=1$ if and only if there exists $v \in V_{k}$ such that $\left(C_{3}\right)^{2} \leq G_{v}$ (see Example 6.13).

(4) $n=10: G \simeq 10 T 7 \simeq A_{5}, G \simeq 10 T 26 \simeq A_{6}$ and $G \simeq 10 T 32 \simeq S_{6}$. By Theorem 6.5, we have Obs $(K / k)=$ $\operatorname{Obs}_{1}(L / K / k)$.

(4-1) The case $G=10 T 7 \simeq A_{5}$. We have $H \simeq S_{3}$ and $H^{a b} \simeq C_{2}$. It follows from Theorem 1.15 that $\operatorname{Ker} \psi_{1} \simeq \operatorname{Ker} \psi_{1} / \varphi_{1}\left(\operatorname{Ker} \psi_{2}^{\text {nr }}\right) \simeq \mathbb{Z} / 2 \mathbb{Z}$. Apply FirstObstructionDr $\left(G, G^{\prime}\right)$ for all subgroups $G^{\prime} \leq G$. We get that $\operatorname{Obs}_{1}(L / K / k)=1$ if and only if there exists $v \in V_{k}$ such that $V_{4} \leq G_{v}$ (see Example 6.14).

(4-2) The case $G=10 T 26 \simeq A_{6}$. We have $H \simeq\left(C_{3}\right)^{2} \rtimes C_{4}$ and $H^{a b} \simeq C_{4}$. Applying FirstobstructionN $(G)$ and FirstObstructionDnr $(G)$, we obtain that $\operatorname{Ker} \psi_{1} \simeq \mathbb{Z} / 4 \mathbb{Z}$ and $\operatorname{Ker} \psi_{1} / \varphi_{1}\left(\operatorname{Ker} \psi_{2}^{\text {nr }}\right) \simeq \mathbb{Z} / 2 \mathbb{Z}$. Apply FirstObstructionDr $\left(G, G^{\prime}\right)$ for all subgroups $G^{\prime} \leq G$. We get that $\operatorname{Obs}_{1}(L / K / k)=1$ if and only if there exists $v \in V_{k}$ such that $D_{4} \leq G_{v}$ (see Example6.14). 
(4-3) The case $G=10 T 32 \simeq S_{6}$. We have $H \simeq\left(S_{3}\right)^{2} \rtimes C_{2}$ and $H^{a b} \simeq C_{2} \times C_{2}$. Applying FirstObstructionN $(G)$ and FirstObstructionDnr $(G)$, we obtain that $\operatorname{Ker} \psi_{1} \simeq \operatorname{Ker} \psi_{1} / \varphi_{1}\left(\operatorname{Ker} \psi_{2}^{\mathrm{nr}}\right) \simeq \mathbb{Z} / 2 \mathbb{Z}$. We also apply the function FirstObstruction $\operatorname{Dr}\left(G, G^{\prime}\right)$ for all subgroups $G^{\prime} \leq G$. We obtain that $\operatorname{Obs}_{1}(L / K / k)=1$ if and only if there exists $v \in V_{k}$ such that (i) $V_{4} \leq G_{v}$ where $N_{\widetilde{G}}\left(V_{4}\right) \simeq C_{8} \rtimes\left(C_{2} \times C_{2}\right)$ for the normalizer $N_{\widetilde{G}}\left(V_{4}\right)$ of $V_{4}$ in $\widetilde{G}$ with the normalizer $\widetilde{G}=N_{S_{10}}(G) \simeq \operatorname{Aut}(G)$ of $G$ in $S_{10}$ (equivalently, $\left|\operatorname{Orb}_{V_{4}}(i)\right|=2$ for any $1 \leq i \leq 10$ ) or (ii) $D_{4} \leq G_{v}$ where $D_{4} \leq[G, G] \simeq A_{6}$ (see Details 6.8 and Example 6.14).

(5) $n=14: G=14 T 30 \simeq \operatorname{PSL}_{2}\left(\mathbb{F}_{13}\right)$. By Theorem 6.5, we obtain that $\operatorname{Obs}(K / k)=\operatorname{Obs}_{1}(L / K / k)$. We have $H \simeq C_{13} \rtimes C_{6}$ and $H^{a b} \simeq C_{6}$. Applying FirstObstructionN $(G)$ and FirstObstructionDnr $(G)$, we obtain that $\operatorname{Ker} \psi_{1} \simeq \mathbb{Z} / 6 \mathbb{Z}$ and $\operatorname{Ker} \psi_{1} / \varphi_{1}\left(\operatorname{Ker} \psi_{2}^{\text {nr }}\right) \simeq \mathbb{Z} / 2 \mathbb{Z}$. Apply FirstObstructionDr $\left(G, G^{\prime}\right)$ for all subgroups $G^{\prime} \leq G$. We get that $\operatorname{Obs}_{1}(L / K / k)=1$ if and only if there exists $v \in V_{k}$ such that $V_{4} \leq G_{v}$ (see Example 6.15).

(6) $n=15: G=15 T 9 \simeq\left(C_{5}\right)^{2} \rtimes C_{3}$ and $G \simeq 15 T 14 \simeq\left(C_{5}\right)^{2} \rtimes S_{3}$. By Theorem 6.5 we obtain that $\operatorname{Obs}(K / k)=\operatorname{Obs}_{1}(L / K / k)$.

(6-1) The case $G=15 T 9 \simeq\left(C_{5}\right)^{2} \rtimes C_{3}$. We have $H \simeq H^{a b} \simeq C_{5}$. Applying FirstObstructionN $(G)$ and FirstObstructionDnr $(G)$, we have $\operatorname{Ker} \psi_{1} \simeq \operatorname{Ker} \psi_{1} / \varphi_{1}\left(\operatorname{Ker} \psi_{2}^{\text {nr }}\right) \simeq \mathbb{Z} / 5 \mathbb{Z}$. Apply FirstObstructionDr $\left(G, G^{\prime}\right)$ for all subgroups $G^{\prime} \leq G$. We get that $\operatorname{Obs}_{1}(L / K / k)=1$ if and only if there exists $v \in V_{k}$ such that $\left(C_{5}\right)^{2} \leq G_{v}$ (see Example 6.16).

(6-2) The case $G=15 T 14 \simeq\left(C_{5}\right)^{2} \rtimes S_{3}$. We have $H \simeq H^{a b} \simeq C_{10}$. Applying FirstObstructionN $(G)$ and FirstObstructionDnr $(G)$, we obtain that $\operatorname{Ker} \psi_{1} \simeq \operatorname{Ker} \psi_{1} / \varphi_{1}\left(\operatorname{Ker} \psi_{2}^{\mathrm{nr}}\right) \simeq \mathbb{Z} / 5 \mathbb{Z}$. We apply the function FirstObstructionDr $\left(G, G^{\prime}\right)$ for all subgroups $G^{\prime} \leq G$. We get that $\operatorname{Obs}_{1}(L / K / k)=1$ if and only if there exists $v \in V_{k}$ such that $\left(C_{5}\right)^{2} \leq G_{v}$ (see Example 6.16).

Remark 6.7. By the proof of Theorem 1.18, for $n=8$ (resp. $n=9$ ), there exists $\widetilde{L} \supset L$ with $[\widetilde{L}: L]=2$ (resp. $[\widetilde{L}: L]=3)$ such that $\operatorname{Obs}(K / k)=\operatorname{Obs}_{1}(\widetilde{L} / K / k)$ although $\operatorname{Obs}(K / k) \neq \operatorname{Obs}_{1}(L / K / k)$ when $L / k$ is unramified and $\operatorname{Obs}_{1}(L / K / k)=1$ except for the case $G=8 T 21$ with $\operatorname{Obs}_{1}(L / K / k) \simeq \mathbb{Z} / 2 \mathbb{Z}$.

Details 6.8 (The cases (1-4) $G=8 T 31,8 T 38,(2) G=8 T m(m=9,11,15,19,22,32)$ and (4-3) $G=10 T 32$ in Theorem 1.18). We take generators of $G$ and $H=\operatorname{Stab}_{1}(G)$ in Theorem 1.18 (Table 2) and give more details for the cases (1-4) $G=8 T 31,8 T 38,(2) G=8 T m(m=9,11,15,19,22,32)$ and (4-3) $G=10 T 32$.

(1-4) For $G=8 T 31 \simeq\left(\left(C_{2}\right)^{4} \rtimes C_{2}\right) \rtimes C_{2}$ and $\left.G^{\prime}=8 T 38 \simeq\left(\left(\left(C_{2}\right)^{4}\right) \rtimes C_{2}\right) \rtimes C_{2}\right) \rtimes C_{3}$, we take $G=$ $\left\langle g_{1}, g_{2}, g_{3}\right\rangle=\operatorname{Syl}_{2}\left(G^{\prime}\right) \triangleleft G^{\prime}=\left\langle g_{1}, g_{2}, g_{3}^{\prime}\right\rangle$ and $H=\operatorname{Stab}_{1}(G)=\left\langle g_{1},(2,6),(3,7)\right\rangle \simeq\left(C_{2}\right)^{3} \leq H^{\prime}=\operatorname{Stab}_{1}\left(G^{\prime}\right)=$ $\left\langle g_{1},(2,6),(2,8,3)(4,7,6)\right\rangle \simeq C_{2} \times A_{4}$ where $g_{1}=(4,8), g_{2}=(1,8)(2,3)(4,5)(6,7), g_{3}=(1,3)(2,8)(4,6)(5,7)$, $g_{3}^{\prime}=(1,2,3)(5,6,7)$. There exist 61 subgroups $V_{4} \leq G$ and 14 of them satisfy $\left|\operatorname{Orb}_{V_{4}}(i)\right|=4(1 \leq i \leq 8)$ :

$$
\begin{aligned}
V_{4}^{(1)} & =\left\{1, \sigma_{1}, \sigma_{2}, \sigma_{3}\right\}, V_{4}^{(2)}=\left\{1, \sigma_{1}, \sigma_{6}, \sigma_{7}\right\}, V_{4}^{(3)}=\left\{1, \tau_{1}, \tau_{2}, \tau_{6}\right\}, V_{4}^{(4)}=\left\{1, \tau_{1}, \sigma_{4}, \tau_{5}\right\}, \\
V_{4}^{(5)} & \left.=\left\{1, \sigma_{2}, \tau_{4}, \sigma_{7}\right\}, V_{4}^{(6)}=\left\{1, \tau_{2}, \sigma_{4}, \sigma_{5}\right)\right\}, V_{4}^{(7)}=\left\{1, \sigma_{3}, \tau_{4}, \sigma_{6}\right\}, V_{4}^{(8)}=\left\{1, \sigma_{5}, \tau_{5}, \tau_{6}\right\}, \\
V_{4}^{(9)} & \left.=\left\{1, \sigma_{1}, \tau_{3}, \tau_{4}\right\}, V_{4}^{(10)}=\left\{1, \tau_{1}, \tau_{3}, \sigma_{5}\right)\right\}, V_{4}^{(11)}=\left\{1, \sigma_{2}, \tau_{3}, \sigma_{6}\right\}, \\
V_{4}^{(12)} & =\left\{1, \tau_{2}, \tau_{3}, \tau_{5}\right\}, V_{4}^{(13)}=\left\{1, \sigma_{3}, \tau_{3}, \sigma_{7}\right\}, V_{4}^{(14)}=\left\{1, \sigma_{4}, \tau_{3}, \tau_{6}\right\}
\end{aligned}
$$

where $\sigma_{1}=(1,2)(3,4)(5,6)(7,8), \sigma_{2}=(1,3)(2,4)(5,7)(6,8), \sigma_{3}=(1,4)(2,3)(5,8)(6,7), \sigma_{4}=(1,4)(2,7)(3,6)(5,8)$, $\sigma_{5}=(1,6)(2,5)(3,4)(7,8), \sigma_{6}=(1,7)(2,8)(3,5)(4,6), \sigma_{7}=(1,8)(2,7)(3,6)(4,5), \tau_{1}=(1,2)(3,8)(4,7)(5,6)$, $\tau_{2}=(1,3)(2,8)(4,6)(5,7), \tau_{3}=(1,5)(2,6)(3,7)(4,8), \tau_{4}=(1,6)(2,5)(3,8)(4,7), \tau_{5}=(1,7)(2,4)(3,5)(6,8)$, $\tau_{6}=(1,8)(2,3)(4,5)(6,7)$. Note that $Z(G)=\left\langle\tau_{3}\right\rangle \simeq C_{2}$ and the first 8 groups $V_{4}^{(i)}(1 \leq i \leq 8)$ satisfy $V_{4} \cap Z(G)=1$ as appearing in Theorem 1.18 (Table 2) and the last 6 groups $V_{4}^{(i)}(9 \leq i \leq 14)$ satisfy 
$V_{4} \cap Z(G) \simeq C_{2}$. On the other hand, there exist 15 subgroups $C_{4} \times C_{2} \leq G$ :

$$
\begin{aligned}
G^{(1)} & =\left\langle(1,4,5,8)(2,3,6,7), \sigma_{1}, \tau_{3}\right\rangle, \\
G^{(2)} & =\left\langle(1,8,5,4)(2,3,6,7), \tau_{1}, \tau_{3}\right\rangle, \\
G^{(3)} & =\left\langle(1,8,5,4)(2,3,6,7),(1,2,5,6)(3,4,7,8), \tau_{3}\right\rangle, \\
G^{(4)} & =\left\langle(1,6,5,2)(3,8,7,4), \tau_{6}, \tau_{3}\right\rangle, \\
G^{(5)} & =\left\langle(1,4,5,8)(2,3,6,7),(1,2,5,6)(3,8,7,4), \tau_{3}\right\rangle, \\
G^{(6)} & =\left\langle(1,6,5,2)(3,4,7,8), \sigma_{3}, \tau_{3}\right\rangle, \\
G^{(7)} & =\langle(1,2)(3,8,7,4)(5,6),(1,5)(2,6),(3,7)(4,8)\rangle, \\
G^{(8)} & =\langle(1,6,5,2)(3,8)(4,7),(1,5)(2,6),(3,7)(4,8)\rangle, \\
G^{(9)} & =\langle(1,6,5,2)(3,8,7,4),(1,5)(2,6),(3,7)(4,8)\rangle, \\
G^{(10)} & =\langle(1,7)(2,4,6,8)(3,5),(1,5)(3,7),(2,6)(4,8)\rangle, \\
G^{(11)} & =\langle(1,7,5,3)(2,8)(4,6),(1,5)(3,7),(2,6)(4,8)\rangle, \\
G^{(12)} & =\langle(1,7,5,3)(2,4,6,8),(1,5)(3,7),(2,6)(4,8)\rangle, \\
G^{(13)} & =\langle(1,4,5,8)(2,3)(6,7),(1,5)(4,8),(2,6)(3,7)\rangle, \\
G^{(14)} & =\langle(1,4)(2,3,6,7)(5,8),(1,5)(4,8),(2,6)(3,7)\rangle, \\
G^{(15)} & =\langle(1,4,5,8)(2,3,6,7),(1,5)(4,8),(2,6)(3,7)\rangle .
\end{aligned}
$$

Note that $[G, G]=\langle(1,5)(4,8),(2,6)(4,8),(2,6)(3,7)\rangle \simeq\left(C_{2}\right)^{3}$ and the first 6 groups $G^{(i)}(1 \leq i \leq 6)$ satisfy $G^{(i)} \cap[G, G]=\left\langle\tau_{3}\right\rangle \simeq C_{2}$ and are transitive in $S_{8}$ as appearing in Theorem 1.18 (Table 2) and the last 9 groups $G^{(i)}$ $(7 \leq i \leq 15)$ satisfy $G^{(i)} \cap[G, G] \simeq V_{4}$ and are not transitive in $S_{8}$. Also, there exist 3 subgroups $\left(C_{2}\right)^{3} \rtimes V_{4} \leq G$. They are all transitive in $S_{8}$ (see Example 6.11).

(2-1) For $G=8 T 9 \simeq D_{4} \times C_{2}$, we take $G=\left\langle g_{1}, g_{2}, g_{3}, g_{4}\right\rangle$ and $H=\operatorname{Stab}_{1}(G)=\left\langle g_{4}\right\rangle \simeq C_{2}$ where $g_{1}=$ $(1,8)(2,3)(4,5)(6,7), g_{2}=(1,3)(2,8)(4,6)(5,7), g_{3}=(1,5)(2,6)(3,7)(4,8), g_{4}=(4,5)(6,7)$. There exist 13 subgroups $V_{4} \leq G$ and 8 of them satisfy $\left|\operatorname{Orb}_{V_{4}}(i)\right|=4(1 \leq i \leq 8)$ :

$$
\begin{aligned}
& V_{4}^{(1)}=\left\{1, \sigma_{1}, \sigma_{3}, \sigma_{6}\right\}, V_{4}^{(2)}=\left\{1, \sigma_{1}, \sigma_{4}, \sigma_{5}\right\}, V_{4}^{(3)}=\left\{1, \sigma_{2}, \sigma_{3}, \sigma_{5}\right\}, V_{4}^{(4)}=\left\{1, \sigma_{2}, \sigma_{4}, \sigma_{6}\right\}, \\
& V_{4}^{(5)}=\left\{1, \sigma_{1}, \sigma_{2}, \tau_{3}\right\}, V_{4}^{(6)}=\left\{1, \sigma_{3}, \sigma_{4}, \tau_{3}\right\}, V_{4}^{(7)}=\left\{1, \sigma_{5}, \sigma_{6}, \tau_{3}\right\}, V_{4}^{(8)}=\left\{1, \tau_{1}, \tau_{2}, \tau_{3}\right\}
\end{aligned}
$$

where $\sigma_{1}=(1,2)(3,8)(4,7)(5,6), \sigma_{2}=(1,3)(2,8)(4,6)(5,7), \sigma_{3}=(1,4)(2,7)(3,6)(5,8), \sigma_{4}=(1,5)(2,6)(3,7)(4,8)$, $\sigma_{5}=\sigma_{1} \sigma_{4}=(1,6)(2,5)(3,4)(7,8), \sigma_{6}=\sigma_{1} \sigma_{3}=(1,7)(2,4)(3,5)(6,8), \tau_{1}=(1,2)(3,8)(4,6)(5,7), \tau_{2}=(1,3)(2,8)$ $(4,7)(5,6), \tau_{3}=(1,8)(2,3)(4,5)(6,7)$. Note that $[G, G]=\left\langle\tau_{3}\right\rangle \simeq C_{2}$ and the first half $V_{4}^{(i)}(1 \leq i \leq 4)$ satisfy $V_{4}^{(i)} \cap[G, G]=1$ as appearing in Theorem 1.18 (Table 2) although the second half $V_{4}^{(i)}(5 \leq i \leq 8)$ satisfy $[G, G] \leq V_{4}^{(i)}$. On the other hand, there exists the unique subgroup $C_{4} \times C_{2} \leq G$ (see Example 6.12).

$(2-2)$ For $G=8 T 11 \simeq\left(C_{4} \times C_{2}\right) \rtimes C_{2}$, we take $G=\left\langle g_{1}, g_{2}, g_{3}\right\rangle$ and $H=\operatorname{Stab}_{1}(G)=\langle(2,6)(4,8)\rangle \simeq C_{2}$ where $g_{1}=(1,5)(3,7), g_{2}=(1,3,5,7)(2,4,6,8), g_{3}=(1,4,5,8)(2,3,6,7)$. There exist 3 subgroups $C_{4} \times C_{2} \leq G$ :

$$
\begin{aligned}
& G^{(1)}=\left\langle g_{2},(1,2)(3,4)(5,6)(7,8)\right\rangle, \\
& G^{(2)}=\left\langle g_{2},(1,8)(2,3)(4,5)(6,7)\right\rangle, \\
& G^{(3)}=\left\langle g_{2},(2,6)(4,8)\right\rangle .
\end{aligned}
$$

The first two groups $G^{(1)}$ and $G^{(2)}$ are transitive in $S_{8}$ as appearing in Theorem 1.18 (Table 2) although the last one $G^{(3)}$ is not transitive in $S_{8}$ (see Example 6.12).

$(2-3)$ For $G=8 T 15 \simeq C_{8} \rtimes V_{4}$, we take $G=\left\langle g_{1}, g_{2}, g_{3}\right\rangle$ and $H=\operatorname{Stab}_{1}(G)=\langle(2,8)(3,7)(4,6),(2,4)(3,7)(6,8)\rangle \simeq$ $V_{4}$ where $g_{1}=(1,2,3,4,5,6,7,8), g_{2}=(1,5)(3,7), g_{3}=(1,6)(2,5)(3,4)(7,8)$. There exist 15 subgroups $V_{4} \leq G$ and 5 of them satisfy $\left|\mathrm{Orb}_{V_{4}}(i)\right|=2(1 \leq i \leq 8)$ :

$$
\begin{aligned}
& V_{4}^{(1)}=\left\{1, \sigma_{1}, \tau_{1}, \tau_{2}\right\}, V_{4}^{(2)}=\left\{1, \sigma_{2}, \tau_{3}, \tau_{4}\right\}, V_{4}^{(3)}=\left\{1, \sigma_{2}, \tau_{5}, \tau_{6}\right\}, V_{4}^{(4)}=\left\{1, \sigma_{1}, \tau_{7}, \tau_{8}\right\}, \\
& V_{4}^{(5)}=\left\{1, \sigma_{1}, \sigma_{2}, \sigma_{1} \sigma_{2}\right\}
\end{aligned}
$$

where $\sigma_{1}=(1,5)(3,7), \sigma_{2}=(2,6)(4,8), \tau_{1}=(2,4)(3,7)(6,8), \tau_{2}=(1,5)(2,4)(6,8), \tau_{3}=(1,3)(4,8)(5,7)$, $\tau_{4}=(1,3)(2,6)(5,7), \tau_{5}=(1,7)(3,5)(4,8), \tau_{6}=(1,7)(2,6)(3,5), \tau_{7}=(2,8)(3,7)(4,6), \tau_{8}=(1,5)(2,8)(4,6)$. Note that $[G, G]=\langle(1,3,5,7),(2,4,6,8)\rangle \simeq C_{4}$ and the first four groups $V_{4}^{(i)}(1 \leq i \leq 4)$ satisfy that $V_{4}^{(i)} \cap$ 
$[G, G]=1$ and $V_{4}$ is not in $A_{8}$ as appearing in Theorem 1.18 (Table 2) although the last one $V_{4}^{(5)}$ satisfy that $V_{4}^{(5)} \cap[G, G] \simeq C_{2}$ and $V_{4}$ is in $A_{8}$. On the other hand, there exist 3 subgroups $C_{4} \times C_{2} \leq G$ :

$$
\begin{aligned}
& G^{(1)}=\langle(1,3,5,7)(2,8,6,4),(1,2)(3,8)(4,7)(5,6)\rangle, \\
& G^{(2)}=\langle(1,3,5,7)(2,8,6,4),(1,4)(2,3)(5,8)(6,7)\rangle, \\
& G^{(3)}=\langle(1,3,5,7)(2,4,6,8),(2,6)(4,8)\rangle .
\end{aligned}
$$

The first two groups $G^{(i)}(i=1,2)$ satisfy $G^{(i)} \cap[G, G] \simeq C_{2}(i=1,2)$ which is transitive in $S_{8}$ as appearing in Theorem 1.18 (Table 2) although the last one $G^{(3)}$ satisfy $G^{(i)} \cap[G, G] \simeq C_{4}$ which is not transitive in $S_{8}$ (see Example 6.12).

$(2-4)$ For $G=8 T 19 \simeq\left(C_{2}\right)^{3} \rtimes C_{4}$, we take $G=\left\langle g_{1}, g_{2}, g_{3}, g_{4}\right\rangle$ and $H=\operatorname{Stab}_{1}(G)=\langle(2,8)(4,5,6,7)\rangle \simeq C_{4}$ where $g_{1}=(1,8)(2,3)(4,5)(6,7), g_{2}=(1,3)(2,8)(4,6)(5,7), g_{3}=(1,5)(2,6)(3,7)(4,8), g_{4}=(1,3)(4,5,6,7)$. There exist 13 subgroups $V_{4} \leq G$ and 8 of them satisfy $\left|\operatorname{Orb}_{V_{4}}(i)\right|=4(1 \leq i \leq 8)$ :

$$
\begin{aligned}
& V_{4}^{(1)}=\left\{1, \sigma_{1}, \sigma_{2}, \sigma_{5}\right\}, V_{4}^{(2)}=\left\{1, \sigma_{1}, \sigma_{3}, \sigma_{4}\right\}, V_{4}^{(3)}=\left\{1, \sigma_{2}, \sigma_{3}, \sigma_{6}\right\}, V_{4}^{(4)}=\left\{1, \sigma_{4}, \sigma_{5}, \sigma_{6}\right\}, \\
& V_{4}^{(5)}=\left\{1, \tau_{1}, \tau_{2}, \tau_{3}\right\}, V_{4}^{(6)}=\left\{1, \tau_{2}, \sigma_{1}, \sigma_{6}\right\}, V_{4}^{(7)}=\left\{1, \tau_{2}, \sigma_{2}, \sigma_{4}\right\}, V_{4}^{(8)}=\left\{1, \tau_{2}, \sigma_{3}, \sigma_{5}\right\}
\end{aligned}
$$

where $\sigma_{1}=(1,2)(3,8)(4,7)(5,6), \sigma_{2}=(1,4)(2,7)(3,6)(5,8), \sigma_{3}=(1,5)(2,6)(3,7)(4,8), \sigma_{4}=(1,6)(2,5)(3,4)(7,8)$, $\sigma_{5}=(1,7)(2,4)(3,5)(6,8), \sigma_{6}=(1,8)(2,3)(4,5)(6,7), \tau_{1}=(1,2)(3,8)(4,5)(6,7), \tau_{2}=(1,3)(2,8)(4,6)(5,7)$, $\tau_{3}=(1,8)(2,3)(4,7)(5,6)$. Note that $Z(G)=\left\langle\tau_{2}\right\rangle \simeq C_{2}$ and the first four groups $V_{4}^{(i)}(1 \leq i \leq 4)$ satisfy $V_{4}^{(i)} \cap Z(G)=1$ and $V_{4}^{(i)} \cap Z^{2}(G) \simeq C_{2}$ with the upper central series $1 \leq Z(G) \leq Z^{2}(G) \leq G$ of $G$ as appearing in Theorem 1.18 (Table 2) although the last four groups $V_{4}^{(i)}(5 \leq i \leq 8)$ satisfy $V_{4}^{(i)} \cap Z(G) \simeq C_{2}$. On the other hand, there exist 5 subgroups $C_{4} \times C_{2} \leq G$ :

$$
\begin{aligned}
& G^{(1)}=\langle(2,8)(4,5,6,7),(1,3)(2,8)\rangle, \\
& G^{(2)}=\langle(1,2,3,8)(5,7),(4,6)(5,7)\rangle, \\
& G^{(3)}=\langle(1,5,3,7)(2,6,8,4),(1,8)(2,3)(4,5)(6,7)\rangle, \\
& G^{(4)}=\langle(1,4,2,5)(4,6,8,7),(1,3)(2,8)(4,6)(5,7)\rangle, \\
& G^{(5)}=\langle(1,4,8,7)(2,5,3,6),(1,3)(2,8)(4,6)(5,7)\rangle .
\end{aligned}
$$

Note that $[G, G]=\langle(1,3)(2,8)(4,6)(5,7),(1,8)(2,3)(4,5)(6,7)\rangle \simeq V_{4}$ and the first two groups $G^{(1)}$ and $G^{(2)}$ are not transitive in $S_{8}$ and the third one $G^{(3)}$ is transitive in $S_{8}$ which satisfy $[G, G] \leq G^{(3)}$ as appearing in Theorem 1.18 (Table 2) although the last two $G^{(4)}$ and $G^{(5)}$ are transitive in $S_{8}$ which satisfy $G^{(i)} \cap[G, G] \simeq C_{2}(4 \leq i \leq 5)$ (see Example 6.12).

$(2-5),(2-6)$ For $G=8 T 22 \simeq\left(C_{2}\right)^{3} \rtimes V_{4}$ and $G^{\prime}=8 T 32 \simeq\left(\left(C_{2}\right)^{3} \rtimes V_{4}\right) \rtimes C_{3}$, we take $G=\left\langle g_{1}, g_{2}, g_{3}, g_{4}, g_{5}\right\rangle$, $H=\operatorname{Stab}_{1}(G)=\left\langle g_{4}, g_{5}\right\rangle \simeq V_{4}, G^{\prime}=\left\langle g_{1}, g_{2}, g_{3}, g_{4}^{\prime}, g_{5}^{\prime}\right\rangle$ and $H^{\prime}=\operatorname{Stab}_{1}\left(G^{\prime}\right)=\left\langle g_{5}^{\prime},(2,3,8)(4,7,5)\right\rangle \simeq A_{4}$ where $g_{1}=(1,8)(2,3)(4,5)(6,7), g_{2}=(1,3)(2,8)(4,6)(5,7), g_{3}=(1,5)(2,6)(3,7)(4,8), g_{4}=(2,3)(4,5), g_{5}=$ $(2,3)(6,7), g_{4}^{\prime}=(1,2,3)(4,6,5), g_{5}^{\prime}=(2,5)(3,4)$. There exist 33 subgroups $V_{4} \leq G$ and 14 of them satisfy $\left|\mathrm{Orb}_{V_{4}}(i)\right|=4(1 \leq i \leq 8)$ :

$$
\begin{aligned}
V_{4}^{(1)} & =\left\{1, \sigma_{1}, \sigma_{3}, \sigma_{5}\right\}, V_{4}^{(2)}=\left\{1, \sigma_{1}, \sigma_{4}, \sigma_{6}\right\}, V_{4}^{(3)}=\left\{1, \tau_{1}, \tau_{2}, \tau_{5}\right\}, V_{4}^{(4)}=\left\{1, \tau_{1}, \tau_{4}, \sigma_{5}\right\}, \\
V_{4}^{(5)} & =\left\{1, \tau_{3}, \tau_{2}, \sigma_{5}\right\}, V_{4}^{(6)}=\left\{1, \tau_{3}, \tau_{4}, \tau_{5}\right\}, V_{4}^{(7)}=\left\{1, \sigma_{2}, \sigma_{3}, \sigma_{6}\right\}, V_{4}^{(8)}=\left\{1, \sigma_{2}, \sigma_{4}, \sigma_{5}\right\}, \\
V_{4}^{(9)} & =\left\{1, \sigma_{1}, \sigma_{2}, \tau_{6}\right\}, V_{4}^{(10)}=\left\{1, \tau_{1}, \tau_{3}, \tau_{6}\right\}, V_{4}^{(11)}=\left\{1, \sigma_{3}, \sigma_{4}, \tau_{6}\right\}, \\
V_{4}^{(12)} & =\left\{1, \tau_{2}, \tau_{3}, \tau_{6}\right\}, V_{4}^{(13)}=\left\{1, \sigma_{5}, \sigma_{6}, \tau_{6}\right\}, V_{4}^{(14)}=\left\{1, \sigma_{5}, \tau_{5}, \tau_{6}\right\}
\end{aligned}
$$

where $\sigma_{1}=(1,2)(3,8)(4,6)(5,7), \sigma_{2}=(1,3)(2,8)(4,7)(5,6), \sigma_{3}=(1,4)(2,6)(3,7)(5,8), \sigma_{4}=(1,5)(2,7)(3,6)(4,8)$, $\sigma_{5}=(1,6)(2,4)(3,5)(7,8), \sigma_{6}=(1,7)(2,5)(3,4)(6,8), \tau_{1}=(1,2)(3,8)(4,7)(5,6), \tau_{2}=(1,3)(2,8)(4,6)(5,7)$ $\tau_{3}=(1,4)(2,7)(3,6)(5,8), \tau_{4}=(1,5)(2,6)(3,7)(4,8), \tau_{5}=(1,7)(2,4)(3,5)(6,8), \tau_{6}=(1,8)(2,3)(4,5)(6,7)$. Note that $Z(G)=\left\langle\tau_{6}\right\rangle \simeq C_{2}$ and the first 8 groups $V_{4}^{(i)}(1 \leq i \leq 8)$ satisfy $V_{4} \cap Z(G)=1$ as appearing in Theorem 1.18 (Table 2 ) and the last 6 groups $V_{4}^{(i)}(9 \leq i \leq 14)$ satisfy $V_{4} \cap Z(G) \simeq C_{2}$. On the other hand, there exist 9 
subgroups $C_{4} \times C_{2} \leq G$ :

$$
\begin{aligned}
& G^{(1)}=\left\langle(1,4,8,5)(2,6,3,7), \sigma_{1}, \tau_{6}\right\rangle, \\
& G^{(2)}=\left\langle(1,6,8,7)(2,5,3,4), \tau_{1}, \tau_{6}\right\rangle, \\
& G^{(3)}=\left\langle(1,2,8,3)(4,6,5,7), \sigma_{3}, \tau_{6}\right\rangle, \\
& G^{(4)}=\left\langle(1,4,8,5)(2,6,3,7),(1,2,8,3)(4,6,5,7), \tau_{6}\right\rangle, \\
& G^{(5)}=\left\langle(1,2,8,3)(4,7,5,6), \tau_{3}, \tau_{6}\right\rangle, \\
& G^{(6)}=\left\langle(1,2,8,3)(4,7,5,6), \sigma_{5}, \tau_{6}\right\rangle, \\
& G^{(7)}=\left\langle(1,2,8,3)(4,6,5,7),(4,5)(6,7), \tau_{6}\right\rangle, \\
& G^{(8)}=\left\langle(1,4,8,5)(2,6,3,7),(2,3)(6,7), \tau_{6}\right\rangle, \\
& G^{(9)}=\left\langle(1,6,8,7)(2,5,3,4),(2,3)(4,5), \tau_{6}\right\rangle .
\end{aligned}
$$

The first 6 groups $G^{(i)}(1 \leq i \leq 6)$ are transitive in $S_{8}$ as appearing in Theorem 1.18 (Table 2) and the last 3 groups $G^{(i)}(7 \leq i \leq 9)$ are not transitive in $S_{8}$ (see Example 6.12).

(4-3) For $G=10 T 32 \simeq S_{6}$, we take $G=\left\langle g_{1}, g_{2}, g_{3}, g_{4}\right\rangle$ and $H=\operatorname{Stab}_{1}(G)=\left\langle g_{4},(3,10)(6,9)(7,8),(2,4)(3,7)\right.$ $(6,9)(8,10)\rangle \simeq\left(S_{3}\right)^{2} \rtimes C_{2}$ where $g_{1}=(1,2,10)(3,4,5)(6,7,8), g_{2}=(1,3,2,6)(4,5,8,7), g_{3}=(1,2)(4,7)(5,8)(9,10)$, $g_{4}=(3,6)(4,7)(5,8)$. There exist 165 subgroups $V_{4}$ of $G$ and $45=\left(\begin{array}{c}10 \\ 2\end{array}\right)$ groups $\langle(a, b)(c, d)(e, f),(a, b)(g, h)(i, j)\rangle$ $\simeq V_{4}(\{a, b, c, d, e, f, g, h, i, j\}=\{1,2,3,4,5,6,7,8,9,10\})$ of them satisfy $N_{\widetilde{G}}\left(V_{4}\right) \simeq C_{8} \rtimes\left(C_{2} \times C_{2}\right)$ where $\widetilde{G}=N_{S_{10}}(G) \simeq \operatorname{Aut}(G)$ is the normalizer of $G$ in $S_{10}$ (equivalently, $\left|\operatorname{Orb}_{V_{4}}(i)\right|=2$ for any $1 \leq i \leq 10$ ) as appearing in Theorem 1.18 (Table 2). There exist 180 subgroups $D_{4}$ of $G$ and 45 groups of them satisfy $D_{4} \leq[G, G] \simeq A_{6}$ (see Example 6.14$)$.

Example 6.9 $\left(G=4 T 2 \simeq V_{4}\right.$ and $\left.G=4 T 4 \simeq A_{4}\right)$.

Case $G=4 T 2 \simeq V_{4}$.

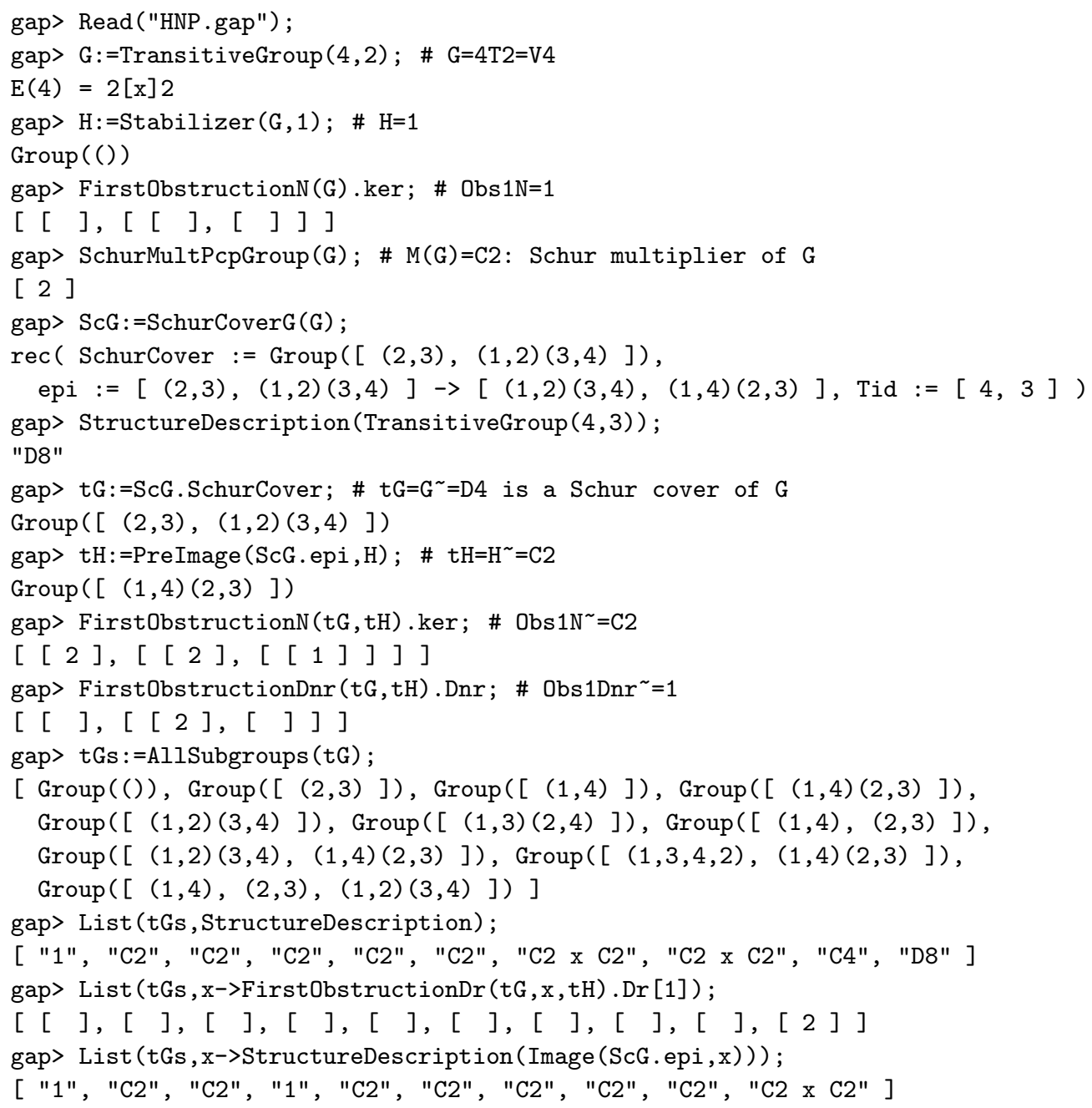


Case $G=4 T 4 \simeq A_{4}$.

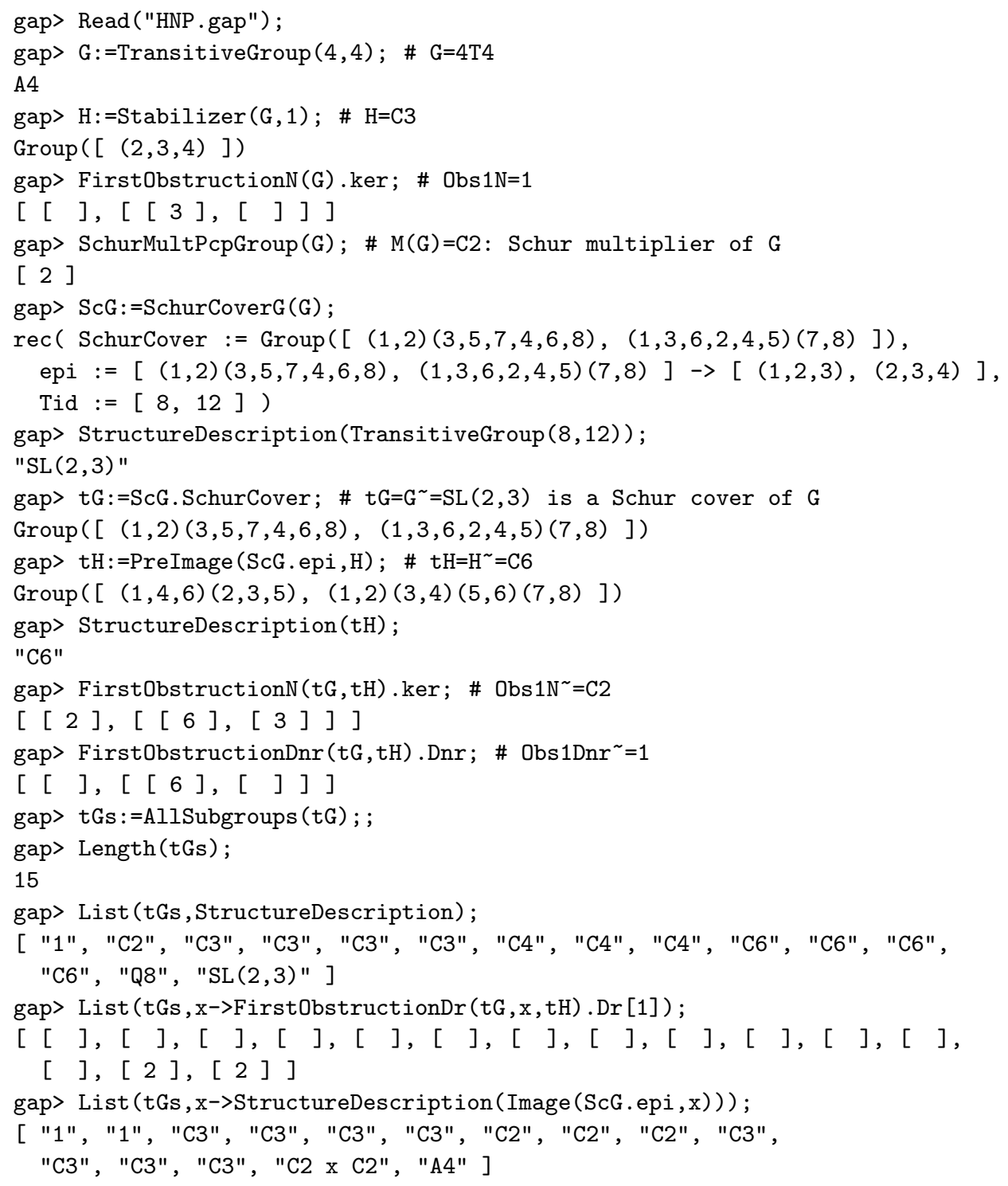

Example 6.10 $\left(G=6 T 4 \simeq A_{4}\right.$ and $\left.G=6 T 12 \simeq A_{5}\right)$.

Case $G=6 T 4 \simeq A_{4}$.

gap> Read ("HNP.gap") ;

gap> G:=TransitiveGroup $(6,4) ; \# \mathrm{G}=6 \mathrm{~T} 4=\mathrm{A} 4$

$A_{-} 4(6)=[2 \sim 2] 3$

gap> $\mathrm{H}:=\operatorname{Stabilizer}(\mathrm{G}, 1) ; \# \mathrm{H}=\mathrm{C} 2$

$\operatorname{Group}([(2,5)(3,6)])$

gap> FirstObstructionN(G).ker; \# Obs1N=C2

[ [ 2 ], [ [ 2 ], [ [ 1 ] ] ] ]

gap> FirstObstructionDnr(G).Dnr; \# Obs1Dnr=1

[ [ ], [ [ 2 ], [ ] ] ]

gap> Gs:=AllSubgroups $(G) ;$;

gap> Length(Gs);

10

gap> List(Gs, StructureDescription);

[ "1", "C2", "C2", "C2", "C3", "C3", "C3", "C3", "C2 x C2", "A4" ]

gap> List (Gs, $\mathrm{x}->$ FirstObstructionDr $(\mathrm{G}, \mathrm{x})$.Dr $[1])$;

$[[],[],[],[],[],[],[],[],[2],[2]]$

Case $G=6 T 12 \simeq A_{5}$.

gap> Read("HNP.gap");

gap> G:=TransitiveGroup $(6,12) ; \# \mathrm{G}=6 \mathrm{~T} 12=\mathrm{A} 5$ 


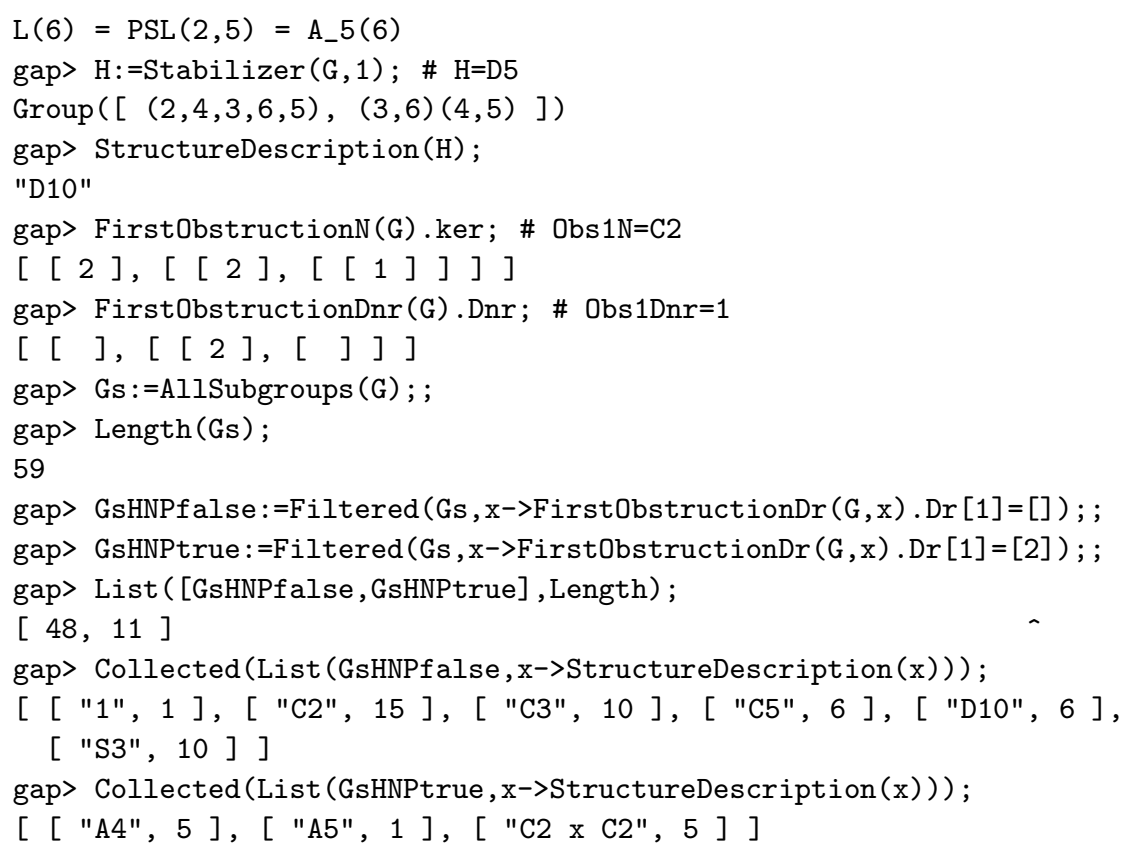

Example 6.11 $(G=8 T m(m=2,3,4,13,14,21,31,37,38))$.

$(1-1) G=8 T 3 \simeq\left(C_{2}\right)^{3}$.

gap> Read("HNP.gap");

gap> G:=TransitiveGroup $(8,3) ; \# \mathrm{G}=8 \mathrm{~T} 3=\mathrm{C} 2 \mathrm{xC} 2 \mathrm{xC} 2$

$\mathrm{E}(8)=2[\mathrm{x}] 2[\mathrm{x}] 2$

gap> $\mathrm{H}:=$ Stabilizer $(\mathrm{G}, 1)$; \# $\mathrm{H}=1$

$\operatorname{Group}(())$

gap> FirstObstructionN(G).ker; \# Obs1N=1

[ [ ], [ [ ], [ ] ] ]

gap> SchurMultPcpGroup $(G)$; \# $M(G)=C 2 \times C 2 \times C 2$ : Schur multiplier of $G$

$[2,2,2]$

gap> ScG: $=$ SchurCoverG $(G)$;

rec $($ SchurCover : $=\operatorname{Group}([(2,3)(4,6)(9,10)(11,13)(12,15)(14,16),(1,2)(3,5)$

$(4,7)(6,8)(10,11)(12,14),(2,4)(3,6)(10,12)(11,14)])$,

epi $:=[(2,3)(4,6)(9,10)(11,13)(12,15)(14,16)$, $(1,2)(3,5)(4,7)(6,8)(10,11)(12,14),(2,4)(3,6)(10,12)(11,14)] \rightarrow$

$[(1,5)(2,6)(3,7)(4,8),(1,3)(2,8)(4,6)(5,7),(1,8)(2,3)(4,5)(6,7)])$

gap> $t G:=S c G . S c h u r C o v e r ; \# t G=G^{\sim}$ is a Schur cover of $G$

$\operatorname{Group}([(2,3)(4,6)(9,10)(11,13)(12,15)(14,16),(1,2)(3,5)(4,7)(6,8)(10,11)$

$(12,14),(2,4)(3,6)(10,12)(11,14)])$

gap> tH: $=$ PreImage $(\mathrm{ScG}$. epi, $\mathrm{H}) ; \# \mathrm{tH}=\mathrm{H}^{\sim}=\mathrm{C} 2 \mathrm{xC} 2 \times \mathrm{xC2}$

Group $([(1,5)(2,3)(4,6)(7,8)(9,13)(10,11)(12,14)(15,16),(9,15)(10,12)(11,14)$

$(13,16),(1,7)(2,4)(3,6)(5,8)])$

gap> IdSmallGroup $(t G)$;

[64, 73 ]

gap> StructureDescription ( $t G)$;

"(C2 x C2 x D8) : C2"

gap> StructureDescription(tH);

"C2 x C2 x C2"

gap> FirstObstructionN ( $t G, t H) . k e r ; \#$ Obs $1 N^{\sim}=\mathrm{C} 2 \times \mathrm{xC} 2 \times \mathrm{xC} 2$

$[[2,2,2],[[2,2,2],[[1,0,0],[0,1,0],[0,0,1]]]]$

gap> FirstObstructionDnr $(\mathrm{tG}, \mathrm{tH}) . \mathrm{Dnr} ; \# \mathrm{Obs} 1 \mathrm{Dnr}{ }^{\sim}=1$

$[$ [ ], [ [ 2, 2, 2], [ ] ] ]

gap> tGs:=AllSubgroups $(t G)$; ;

gap> Length(tGs);

317

gap> Collected (List (tGs, $x->$ FirstObstructionDr ( $t G, x, t H) . D r)$ ) ;

$[[[[],[[2,2,2],[]]], 213]$,

$[[[2],[[2,2,2],[[0,0,1]]]], 29]$,

$[[[2],[[2,2,2],[[0,1,0]]]], 29]$, 
$[[[2],[[2,2,2],[[0,1,1]]]], 5]$,

$[[[2],[[2,2,2],[[1,0,0]]]], 29]$,

$[[[2],[[2,2,2],[[1,0,1]]]], 5]$,

$[[[2],[[2,2,2],[[1,1,0]]]], 5]$,

[ [ [ 2 ] $[[2,2,2],[[1,1,1]]]], 1]$,

$[[[2,2,2],[[2,2,2],[[1,0,0]$,

$[0,1,0],[0,0,1]]]], 1]]$

gap $>$ tGsHNPfalse $0:=$ Filtered (tGs, $\mathrm{x}->$ FirstObstructionDr $(\mathrm{tG}, \mathrm{x}, \mathrm{tH}) . \operatorname{Dr}[1]=[])$; ;

gap> Length(tGsHNPfalse0);

213

gap $>$ tGsHNPtrue $0:=$ Filtered (tGs,$x->$ FirstObstructionDr $(t G, x, t H) . \operatorname{Dr}[1]=[2,2,2]) ;$;

gap> Length (tGsHNPtrue0);

1

gap> Collected (List(tGsHNPfalse0, $\mathrm{x}$->StructureDescription(Image(ScG.epi, $\mathrm{x}$ )))) ;

[ [ "1", 16 ], [ "C2", 197] ]

gap> Collected (List (tGsHNPtrue0, $\mathrm{x}$->StructureDescription(Image (ScG.epi, $\mathrm{x}$ ))) );

[ [ "C2 x C2 x C2", 1 ] ]

$(1-2) G=8 T 21 \simeq\left(C_{2}\right)^{3} \rtimes C_{4}$.

gap> Read ("HNP.gap");

gap $>\mathrm{G}:=$ TransitiveGroup $(8,21) ; \# \mathrm{G}=8 \mathrm{~T} 21=(\mathrm{C} 2 \times \mathrm{CC} 2 \mathrm{xC2}): \mathrm{C} 4$

$1 / 2[2 \wedge 4] \mathrm{E}(4)=[1 / 4 . \mathrm{dD}(4) \wedge 2] 2$

gap> $\mathrm{H}:=\mathrm{Stabilizer}(\mathrm{G}, 1) ; \quad \# \mathrm{H}=\mathrm{C} 2 \mathrm{xC} 2$

Group $([(2,6)(4,8),(3,7)(4,8)])$

gap> FirstObstructionN (G) .ker; \# Obs $1 \mathrm{~N}=\mathrm{C} 2$

[ [ 2 ], [ [ 2,2 ], [ [ 1,0$]]]]$

gap> FirstObstructionDnr(G).Dnr; \# Obs1Dnr=1 $\Rightarrow$ Obs=0bs1=C2 if unramified

[ [ ], [ [ 2,2 ], [ ] ] ]

gap> KerResH3Z(G,H); \# Obs=Obs1

$[[],[[2,2],[]]]$

gap> Gs: =AllSubgroups (G); ;

gap> Length(Gs);

50

gap> GsHNPfalse:=Filtered(Gs, $\mathrm{x}->$ FirstObstructionDr (G,X,H) . $\operatorname{Dr}[1]=[]$ ); ;

gap> Length (GsHNPfalse);

49

gap> GsHNPtrue:=Filtered(Gs, $\mathrm{x}->$ FirstObstructionDr (G, $\mathrm{x}, \mathrm{H})$. $\operatorname{Dr}[1]=[2]$ ); ;

gap> Length(GsHNPtrue);

1

gap> Collected(List (GsHNPfalse, StructureDescription));

[ [ "(C4 x C2): C2", 2$],[$ "1", 1$]$, [ "C2", 11$],[$ "C2 x C2", 13$]$,

[ "C2 x C2 x C2", 2 ], [ "C2 x D8", 1 ], [ "C4", 10 ], [ "C4 x C2", 5 ],

[ "D8", 4 ] ]

gap> Collected (List (GsHNPtrue, StructureDescription));

$[$ [ "(C2 x C2 x C2) : $\mathrm{C} 4 ", 1]]$

(1-3-1) $G=8 T 2 \simeq C_{4} \times C_{2}$.

gap> Read ("HNP.gap");

gap> G:=TransitiveGroup $(8,2) ; \# \mathrm{G}=8 \mathrm{~T} 2=\mathrm{C} 4 \mathrm{xC} 2$

$4[\mathrm{x}] 2$

gap> H:=Stabilizer $(\mathrm{G}, 1) ; \# \mathrm{H}=1$

$\operatorname{Group}(())$

gap> FirstObstructionN(G).ker; \# Obs $1 \mathrm{~N}=1$

$\left.\left[\begin{array}{ll}{[} & ],[\end{array}\right],[\mathrm{l}]\right]$

gap> SchurMultPcpGroup $(G) ; \# M(G)=C 2$ : Schur multiplier of $G$

[ 2 ]

gap> ScG: $=$ SchurCoverG $(G)$;

rec( SchurCover := Group $([(2,4)(3,6),(1,2,5,3)(4,7,6,8)])$,

Tid $:=[8,10]$, epi $:=[(2,4)(3,6),(1,2,5,3)(4,7,6,8)] \rightarrow$

$[(1,5)(2,6)(3,7)(4,8),(1,2,3,8)(4,5,6,7)])$

gap> $\mathrm{tG}:=\mathrm{ScG}$.SchurCover; \# $\mathrm{tG}=\mathrm{G}^{\sim}=(\mathrm{C} 4 \mathrm{xC2}): \mathrm{C} 2$ is a Schur cover of $\mathrm{G}$

Group $([(2,4)(3,6),(1,2,5,3)(4,7,6,8)])$

gap> StructureDescription (TransitiveGroup $(8,10)$ ); 


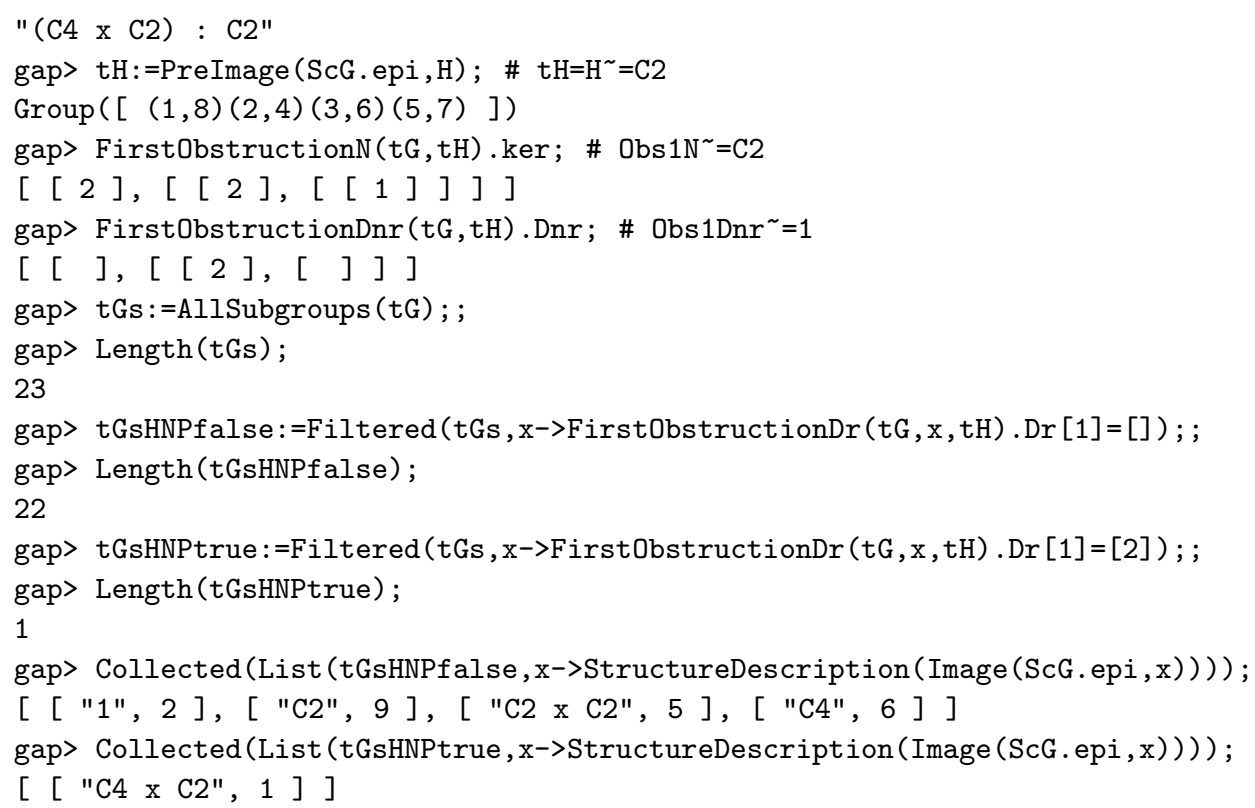


$(1-3-3) G=8 T 13 \simeq A_{4} \times C_{2}$.

gap> Read ("HNP.gap");

gap> G:=TransitiveGroup $(8,13) ; \# \mathrm{G}=8 \mathrm{~T} 13=\mathrm{A} 4 \mathrm{xC2}$

$E(8): 3=A(4)[x] 2$

gap> $\mathrm{H}:=$ Stabilizer $(\mathrm{G}, 1) ; \# \mathrm{H}=\mathrm{C} 3$

Group ( $[(2,3,8)(4,7,5)])$

gap> FirstObstructionN(G).ker; \# Obs1N=1

$[$ [ ], [ [ 3$],[]]]$

gap> SchurMultPcpGroup(G); \# M(G)=C2: Schur multiplier of $G$

[ 2 ]

gap> ScG: $=$ SchurCoverG (G);

rec $($ SchurCover : $=\operatorname{Group}([(1,2,3)(4,6,7)(5,8,9)(10,14,15)(11,16,17)(12,18,19)$

$(13,20,21)(22,23,24),(2,4)(3,5)(6,10)(7,11)(8,12)(9,13)(15,18)(16,22)(17,20)$

$(19,23)])$, Tid $:=[24,21]$, epi $:=[(1,2,3)(4,6,7)(5,8,9)(10,14,15)$

$(11,16,17)(12,18,19)(13,20,21)(22,23,24),(2,4)(3,5)(6,10)(7,11)(8,12)(9,13)$

$(15,18)(16,22)(17,20)(19,23)] \rightarrow[(2,8,3)(4,5,7),(1,5)(2,6)(3,7)(4,8)])$

gap> $t G:=S c G$. SchurCover; \# $t G=G^{\sim}$ is a Schur cover of $G$

Group $([(1,2,3)(4,6,7)(5,8,9)(10,14,15)(11,16,17)(12,18,19)(13,20,21)(22,23,24)$, $(2,4)(3,5)(6,10)(7,11)(8,12)(9,13)(15,18)(16,22)(17,20)(19,23)])$

gap> tH: $=$ PreImage (ScG.epi, H); \# $\mathrm{tH}=\mathrm{H}^{\sim}=\mathrm{C} 6$

Group $([(1,3,2)(4,7,6)(5,9,8)(10,15,14)(11,17,16)(12,19,18)(13,21,20)(22,24,23)$, $(1,24)(2,22)(3,23)(4,16)(5,19)(6,17)(7,11)(8,12)(9,18)(10,20)(13,15)(14,21)])$

gap> StructureDescription(TransitiveGroup $(24,21))$;

"( (C4 x C2) : C2) : C3"

gap> StructureDescription(tH);

"C6"

gap> FirstObstructionN $(\mathrm{tG}, \mathrm{tH}) \cdot \mathrm{ker} ; \# \mathrm{Obs} 1 \mathrm{~N}^{\sim}=\mathrm{C} 2$

[ [ 2 ], [ [ 6 ], [ [ 3$]]]]$

gap> FirstObstructionDnr (tG,tH).Dnr; \# Obs1Dnr ${ }^{\sim}=1$

$[[],[[6],[]]]$

gap> tGs:=AllSubgroups $(t G) ;$;

gap> Length(tGs);

37

gap> tGsHNPfalse:=Filtered(tGs, $\mathrm{x}->$ FirstObstructionDr (tG , x, tH) . Dr [1] $=[]$ ) ; ;

gap> Length (tGsHNPfalse);

30

gap> tGsHNPtrue: $=$ Filtered (tGs,$x->$ FirstObstructionDr $(t G, x, t H) . \operatorname{Dr}[1]=[2]) ;$; gap> Length(tGsHNPtrue);

7

gap> Collected (List(tGsHNPfalse, $\mathrm{x}->$ StructureDescription (Image (ScG.epi, $\mathrm{x}$ )))) ;

[ [ "1", 2$]$, [ "C2", 13 ], [ "C2 x C2", 3 ], [ "C3", 8 ], [ "C6", 4 ] ]

gap> Collected (List(tGsHNPtrue, $\mathrm{x}->$ StructureDescription (Image (ScG.epi,x))));

[ [ "A4", 1 ] , [ "C2 x A4", 1 ], [ "C2 x C2", 4 ], [ "C2 x C2 x C2", 1 ] ]

gap> pi : =NaturalHomomorphismByNormalSubgroup (G, Centre (G));

$[(1,8)(2,3)(4,5)(6,7),(1,3)(2,8)(4,6)(5,7),(1,5)(2,6)(3,7)(4,8)$,

$(1,2,3)(4,6,5)] \rightarrow[f 3, f 2, f 2 * f 3, f 1 * f 2 * f 3]$

gap> Collected (List (tGsHNPtrue, $x->$ StructureDescription (Image (pi, Image (ScG .epi, $x$ ))))) ;

[ [ "A4", 2$],[$ "C2 x C2", 5 ] ]

gap> Collected (List (tGsHNPfalse, $x$->StructureDescription (Image (pi , Image (ScG.epi, $x$ ))))) ;

[ [ "1", 3$],$ [ "C2", 15$],$ [ "C3", 12$]]$

gap> Collected (List (tGsHNPtrue, $\mathrm{x}->$ StructureDescription (Image (pi, Image (ScG .epi, $\mathrm{x}$ )))) ) ;

[ [ "A4", 2$],[$ "C2 x C2", 5 ] ]

$(1-3-4) G=8 T 14 \simeq S_{4}$.

gap> Read ("HNP.gap") ;

gap> G:=TransitiveGroup $(8,14) ; \# \mathrm{G}=8 \mathrm{~T} 14=\mathrm{S} 4$

$\mathrm{S}(4)[1 / 2] 2=1 / 2\left(\mathrm{~S}_{-} 4[\mathrm{x}] 2\right)$

gap> $\mathrm{H}:=$ Stabilizer $(\mathrm{G}, 1)$; \# H=C3

$\operatorname{Group}([(2,8,3)(4,7,6)])$

gap> FirstObstructionN(G).ker; \# Obs1N=C3

[ [ 3$],\left[\left[\begin{array}{l}3 \\ ]\end{array},\left[\left[\begin{array}{lll}1 & 1\end{array}\right]\right]\right]\right.$

gap> FirstObstructionDnr(G).Dnr; \# 0sb1Dr=C3 
[ [ 3$],[[3],[[1]]]]$

gap> SchurMultPcpGroup $(G) ; \# M(G)=C 2$ : Schur multiplier of $G$

[ 2 ]

gap> ScG: $=$ SchurCoverG $(G)$;

rec( SchurCover := Group $([(2,4)(3,6)(5,8),(1,2,5,7,4,3)(6,8)])$, Tid := $[8,23]$,

epi $:=[(2,4)(3,6)(5,8),(1,2,5,7,4,3)(6,8)] \rightarrow$

$[(1,4)(2,6)(3,7)(5,8),(2,8,3)(4,7,6)])$

gap> $\mathrm{tG}:=\mathrm{ScG}$.SchurCover; $\# \mathrm{tG}=\mathrm{G}^{\sim}=\mathrm{SL}(2,3)$ is a Schur cover of $\mathrm{G}$

Group $([(2,4)(3,6)(5,8),(1,2,5,7,4,3)(6,8)])$

gap> tH:=PreImage $(\mathrm{ScG}$. epi, $\mathrm{H}) ; \# \mathrm{tH}=\mathrm{H}^{\sim}=\mathrm{C} 6$

$\operatorname{Group}([(1,4,5)(2,3,7),(1,7)(2,4)(3,5)(6,8)])$

gap> StructureDescription $(t G)$;

"GL $(2,3)$ "

gap> StructureDescription(tH);

"C6"

gap> FirstObstructionN (tG,tH).ker; \# Obs $1 \mathrm{~N}^{\sim}=\mathrm{C} 6$

[ [ 6 ], [ [ 6$],[[1]]]]$

gap> FirstObstructionDnr $(\mathrm{tG}, \mathrm{tH}) . \mathrm{Dnr} ; \#$ Obs1Dnr $\sim=\mathrm{C} 3$

[ [ 3$],[$ [ 6$],[[2]]]]$

gap> tGs:=AllSubgroups $(t G) ;$;

gap> Length(tGs);

55

gap> tGsHNPfalse1:=Filtered(tGs, $\mathrm{x}->$ FirstObstructionDr $(\mathrm{tG}, \mathrm{x}, \mathrm{tH}) \cdot \operatorname{Dr}[1]=[])$; ;

gap> tGsHNPfalse2:=Filtered(tGs, $\mathrm{x}->$ FirstObstructionDr ( $\mathrm{tG}, \mathrm{x}, \mathrm{tH}) . \operatorname{Dr}[1]=[3])$; ;

gap> tGsHNPtrue $1:=$ Filtered ( $t G s, x->$ FirstObstructionDr $(t G, x, t H) . \operatorname{Dr}[1]=[2])$; ;

gap> tGsHNPtrue $2:=F i l t e r e d(t G s, x->F i r s t O b s t r u c t i o n D r(t G, x, t H) . D r[1]=[6])$; ;

gap> List ([tGsHNPfalse1, tGsHNPfalse2, tGsHNPtrue1, tGsHNPtrue2] , Length) ;

$[26,20,7,2]$

gap> Sum(last);

55

gap> Collected (List(tGsHNPfalse1, $\mathrm{x}->$ StructureDescription(Image (ScG.epi,x)))) ;

[ [ "1", 2 ], [ "C2", 21$],[$ "C4", 3 ] ]

gap> Collected(List(tGsHNPfalse2, $\mathrm{x}->$ StructureDescription(Image (ScG.epi,x)))) ;

[ [ "C3", 8 ], [ "S3", 12 ] ]

gap> Collected (List (tGsHNPtrue1, x->StructureDescription(Image (ScG.epi,x)))) ;

[ [ "C2 x C2", 4], [ "D8", 3 ] ]

gap> Collected (List (tGsHNPtrue2, $\mathrm{x}$->StructureDescription(Image (ScG.epi, x)))) ;

[ [ "A4", 1 ], [ "S4", 1 ] ]

$(1-3-5) G=8 T 37 \simeq \operatorname{PSL}_{3}\left(\mathbb{F}_{2}\right) \simeq \operatorname{PSL}_{2}\left(\mathbb{F}_{7}\right)$

gap> Read ("HNP.gap") ;

gap> G:=TransitiveGroup $(8,37) ; \quad \# \mathrm{G}=8 \mathrm{~T} 37=\operatorname{PSL}(3,2)=\operatorname{PSL}(2,7)$

$\mathrm{L}(8)=\mathrm{PSL}(2,7)$

gap> $\mathrm{H}:=$ Stabilizer $(\mathrm{G}, 1) ; \# \mathrm{H}=\mathrm{C} 7: \mathrm{C} 3$

Group $([(2,3,6)(5,8,7),(3,7,8)(4,5,6)])$

gap> StructureDescription $(\mathrm{H})$;

"C7 : C3"

gap> FirstObstructionN(G).ker; \# Obs1N=C3

[ [ 3$],[[3],[[1]]]]$

gap> FirstObstructionDnr(G).Dnr; \# Obs1Dnr=C3

[ [ 3 ], [ [ 3 ], [ [ 11$]]]]$

gap> SchurMultPcpGroup $(G) ; \# M(G)=C 2$ : Schur multiplier of $G$

[ 2 ]

gap> ScG: $=$ SchurCoverG $(G)$;

rec( SchurCover : $=\operatorname{Group}([(1,2,4,8)(3,6,9,12)(5,10,14,11)(7,13,15,16)$,

$(1,3,7,4,9,15)(2,5,11,8,14,10)(6,12)(13,16)])$, Tid := [ 16, 715 ],

epi $:=[(1,2,4,8)(3,6,9,12)(5,10,14,11)(7,13,15,16)$,

$(1,3,7,4,9,15)(2,5,11,8,14,10)(6,12)(13,16)] \rightarrow$

$[(1,2)(3,5)(4,7)(6,8),(2,6,7)(3,5,4)])$

gap> $t G:=S c G$.SchurCover; \# $t G=G=S L(2,7)$ is a Schur cover of $G$

Group $([(1,2,4,8)(3,6,9,12)(5,10,14,11)(7,13,15,16),(1,3,7,4,9,15)$

$(2,5,11,8,14,10)(6,12)(13,16)])$

gap> tH:=PreImage $(\mathrm{ScG}$. epi, $\mathrm{H}) ; \# \mathrm{tH}=\mathrm{H}^{\sim}=\mathrm{C} 2 \mathrm{x}(\mathrm{C} 7: \mathrm{C} 3)$ 


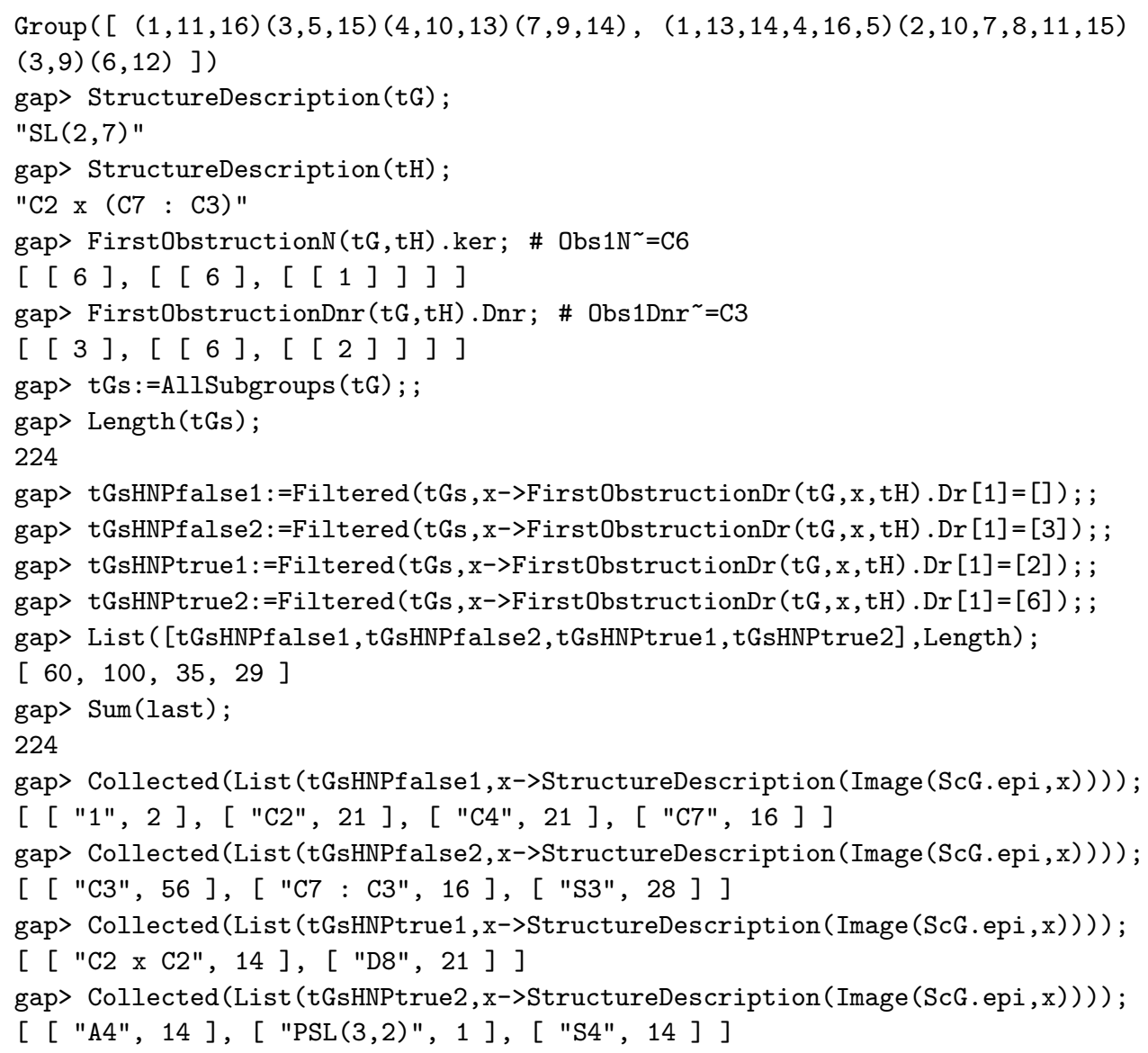


[ [ 2$],[[2,2,2],[[1,1,1]]]]$

[ [ 2$],[[2,2,2],[[1,1,1]]]]$

gap> for $c G$ in cGs do

$>\mathrm{bG}:=\mathrm{cG}$. MinimalStemExtension;

$>\mathrm{bH}:=$ PreImage (cG.epi, $\mathrm{H})$;

$>$ Print (FirstObstructionN (bG,bH).ker [1]);

$>$ Print (FirstObstructionDnr (bG,bH).Dnr [1] );

$>\operatorname{Print}(" \backslash \mathrm{n} ")$;

$>$ od;

$[2,2,2][2,2]$

$[2,2][2,2]$

$[2,2][2,2]$

$[2,2][2,2]$

$[2,2][2,2]$

$[2,2][2,2]$

$[2,2][2,2]$

$[2,2][2,2]$

$[2,2][2,2]$

$[2,2][2,2]$

$[2,2][2,2]$

$[2,2][2,2]$

$[2,2][2,2]$

$[2,2][2,2]$

$[2,2][2,2]$

gap>cG:=cGs $[1] ;$;

gap> bG:=cG.MinimalStemExtension; \# bG=G- is a minimal stem extension of $G$ <permutation group of size 128 with 7 generators>

gap> bH:=PreImage (cG.epi,H); \# bH=H-

<permutation group of size 16 with 4 generators>

gap> FirstObstructionN (bG,bH).ker; \# Obs1N-=C2xC2xC2

$[[2,2,2]$,

$[[2,2,2,2],[[1,0,1,0],[0,1,1,0],[0,0,0,1]]]]$

gap> FirstObstructionDnr (bG,bH).Dnr; \# Obs1Dnr-=C2xC2

$[[2,2],[[2,2,2,2],[[1,0,1,0],[0,1,1,0]]]]$

gap> bGs:=AllSubgroups $(\mathrm{bG})$; ;

gap> Length(bGs);

896

gap> bGsHNPfalse:=Filtered(bGs, $\mathrm{x}->$ Filtered(FirstObstructionDr(bG, $\mathrm{x}, \mathrm{bH})$.Dr [2] [2] ,

$>\mathrm{y}->\mathrm{y}[4]=1)=[]) ;$;

gap> Length (bGsHNPfalse);

855

gap> bGsHNPtrue:=Filtered(bGs, $\mathrm{x}->$ Filtered (FirstObstructionDr (bG, $\mathrm{x}, \mathrm{bH})$. Dr [2] [2] ,

$>\mathrm{y}->\mathrm{y}[4]=1)<>[]) ;$;

gap> Length(bGsHNPtrue);

41

gap> Collected (List (bGsHNPfalse, $x->$ StructureDescription(Image (cG.epi,x)))) ;

[ [ "(C2 x C2 x C2 x C2) : C2", 19$],[$ "(C4 x C2) : C2", 45 ], [ "1", 2 ],

[ "C2", 73 ], [ "C2 x C2", 241$],[$ "C2 x C2 x C2", 154 ],

[ "C2 x C2 x C2 x C2", 17 ], [ "C2 x D8", 57 ], [ "C4", 54 ],

[ "C4 x C2", 45 ], [ "D8", 146 ], [ "Q8", 2 ] ]

gap> Collected(List (bGsHNPtrue, $x->$ StructureDescription (Image (cG.epi, $x)))$ );

$[$ [ $((\mathrm{C} 2 \times \mathrm{C} 2 \times \mathrm{C} 2 \times \mathrm{C} 2): \mathrm{C} 2): \mathrm{C} 2 ", 1]$,

[ "(C2 x C2 x C2) : (C2 x C2)", 1 ], [ "(C2 x C2 x C2) : C4", 11$]$,

[ "(C4 x C2) : C2", 6 ], [ "C2 x C2", 8 ], [ "C2 x C2 x C2", 2 ],

[ "C2 x D8", 6 ], [ "C4 $\mathrm{x}$ C2", 6 ] ]

gap> GsHNPfalse:=Set (bGsHNPfalse, $x->\operatorname{Image}(c G . e p i, x)) ;$;

gap> Length(GsHNPfalse);

192

gap> GsHNPtrue:=Set (bGsHNPtrue, $\mathrm{x}->\operatorname{Image}(\mathrm{cG}$. epi, $\mathrm{x})$ ) ; ;

gap> Length(GsHNPtrue);

33

gap> Intersection(GsHNPfalse, GsHNPtrue);

[ ] 
gap> GsHNPtrueMin:=Filtered (GsHNPtrue, $\mathrm{x}->$ Length (Filtered (GsHNPtrue,

$>\mathrm{y}->\operatorname{IsSubgroup}(\mathrm{x}, \mathrm{y})))=1)$;

[ $\operatorname{Group}([(),(1,5)(2,6),(2,6)(4,8),(2,6)(3,7),(1,6)(2,5)(3,8)(4,7),(1,3)$

$(2,8,6,4)(5,7)]), \operatorname{Group}([(),(1,5)(2,6),(2,6)(4,8),(2,6)(3,7),(1,3)$

$(2,8)(4,6)(5,7),(1,8,5,4)(2,3)(6,7)]), \operatorname{Group}([(),(1,5)(2,6),(2,6)$

$(4,8),(2,6)(3,7),(1,8,5,4)(2,3,6,7),(1,3)(2,8,6,4)(5,7)]), \operatorname{Group}([(1,3)$

$(2,4)(5,7)(6,8),(),(1,4)(2,3)(5,8)(6,7)]), \operatorname{Group}([(1,7,5,3)$

$(2,8,6,4),(),(1,8,5,4)(2,7,6,3),(1,5)(2,6)(3,7)(4,8)]), \operatorname{Group}([(1,7)$

$(2,8)(3,5)(4,6),(),(1,8)(2,7)(3,6)(4,5)]), \operatorname{Group}([(1,3)(2,8)(4,6)$

$(5,7),(),(1,8)(2,3)(4,5)(6,7)]), \operatorname{Group}([(1,7,5,3)(2,4,6,8),(),(1,8,5$,

4) $(2,3,6,7),(1,5)(2,6)(3,7)(4,8)]), \operatorname{Group}([(1,7)(2,4)(3,5)$

$(6,8),(),(1,4)(2,7)(3,6)(5,8)]), \operatorname{Group}([(1,3)(2,4)(5,7)(6,8),(),(1,8$, $5,4)(2,3,6,7),(1,5)(2,6)(3,7)(4,8)]), \operatorname{Group}([(1,7,5,3)(2,8,6,4),(),(1$, 4) $(2,7)(3,6)(5,8),(1,5)(2,6)(3,7)(4,8)]), \operatorname{Group}([(1,3)(2,8)(4,6)$

$(5,7),(),(1,8,5,4)(2,7,6,3),(1,5)(2,6)(3,7)(4,8)]), \operatorname{Group}([(1,7,5,3)$

$(2,4,6,8),(),(1,4)(2,3)(5,8)(6,7),(1,5)(2,6)(3,7)(4,8)]), \operatorname{Group}([(1,3)$

$(2,4)(5,7)(6,8),(),(1,8)(2,7)(3,6)(4,5)]), \operatorname{Group}([(1,3)(2,8)(4,6)$

$(5,7),(),(1,4)(2,7)(3,6)(5,8)]), \operatorname{Group}([(1,7)(2,8)(3,5)(4,6),(),(1,4)$

$(2,3)(5,8)(6,7)]), \operatorname{Group}([(1,7)(2,4)(3,5)(6,8),(),(1,8)(2,3)(4,5)$

$(6,7)]$ ] ]

gap> Length(GsHNPtrueMin);

17

gap> List (GsHNPtrueMin, IdSmallGroup);

$[[32,6],[32,6],[32,6],[4,2],[8,2],[4,2],[4,2]$,

$[8,2],[4,2],[8,2],[8,2],[8,2],[8,2],[4,2]$,

$[4,2],[4,2],[4,2]]$

gap $>$ Collected (List (GsHNPfalse, $x->$ Filtered (GsHNPtrueMin, $y->\operatorname{IsSubgroup}(x, y))$ )) ;

[ [ [ ], 192 ] ]

gap> Gs:=AllSubgroups $(G) ;$;

gap> Length(Gs);

225

gap> GsC2xC2:=Filtered(Gs,x->IdSmallGroup $(x)=[4,2]) ;$;

gap> Length (GsC2xC2);

61

gap> GsC4xC2:=Filtered(Gs, x->IdSmallGroup $(\mathrm{x})=[8,2])$; ;

gap> Length(GsC4xC2);

15

gap> Gs32_6: $=$ Filtered (Gs, $x->\operatorname{IdSmallGroup}(\mathrm{x})=[32,6])$; ;

gap> Length(Gs32_6);

3

gap> GsHNPfalseC2xC2: $=$ Filtered(GsHNPfalse, $\mathrm{x}->\operatorname{IdSmallGroup}(\mathrm{x})=[4,2])$;

$[\operatorname{Group}([(3,7)(4,8),(3,7)]), \operatorname{Group}([(2,6)(4,8),(2,6)(4,8),(2,6)])$,

$\operatorname{Group}([(2,6)(3,7),(2,6)(3,7)(4,8)]), \operatorname{Group}([(1,5)(4,8),(1,5)$

$(4,8),(4,8)]), \operatorname{Group}([(1,5)(3,7),(4,8)]), \operatorname{Group}([(1,5)(2,6)$

$(4,8),(1,5)(2,6)]), \operatorname{Group}([(1,5)(2,6)(3,7)(4,8),(1,5)(2,6)(3,7)$

$(4,8),(4,8)]), \operatorname{Group}([(2,6)(3,7),(2,6)(3,7),(2,6)]), \operatorname{Group}([(2,6)$

$(4,8),(2,6)(4,8),(3,7)]), \operatorname{Group}([(1,5)(3,7),(1,5)(3,7),(3,7)])$,

$\operatorname{Group}([(3,7),(1,5)(4,8)]), \operatorname{Group}([(1,5)(2,6),(3,7)]), \operatorname{Group}([(1,5)$

$(2,6)(3,7)(4,8),(1,5)(2,6)(3,7)(4,8),(3,7)]), \operatorname{Group}([(3,7)$

$(4,8),(2,6)]), \operatorname{Group}([(3,7)(4,8),(2,6)(3,7)]), \operatorname{Group}([(1,2)(3,4)(5,6)$

$(7,8),(3,7)(4,8),(3,7)(4,8)]), \operatorname{Group}([(3,7)(4,8),(1,5)])$,

$\operatorname{Group}([(1,5)(4,8),(1,5)(4,8),(3,7)(4,8)]), \operatorname{Group}([(1,5)(2,6)(3,7)$

$(4,8),(1,5)(2,6)(3,7)(4,8),(3,7)(4,8)]), \operatorname{Group}([(1,5)(2,6)(4,8),(3,7)$

$(4,8)]), \operatorname{Group}([(1,6)(2,5)(3,8)(4,7),(3,7)(4,8),(3,7)(4,8)])$,

$\operatorname{Group}([(1,5)(2,6),(1,5)]), \operatorname{Group}([(1,5)(4,8),(2,6)]), \operatorname{Group}([(1,5)$

$(3,7),(1,5)(3,7),(2,6)]), \operatorname{Group}([(1,5)(2,6)(3,7)(4,8),(1,5)(2,6)(3,7)$

$(4,8),(2,6)]), \operatorname{Group}([(1,3)(2,8)(4,6)(5,7),(2,6)(4,8),(2,6)(4,8)])$,

$\operatorname{Group}([(2,6)(4,8),(2,6)(4,8),(1,5)]), \operatorname{Group}([(1,5)(4,8),(1,5)$

$(4,8),(1,5)(2,6)]), \operatorname{Group}([(1,5)(2,6)(3,7)(4,8),(1,5)(2,6)(3,7)$

$(4,8),(2,6)(4,8)]), \operatorname{Group}([(2,6)(4,8),(2,6)(4,8),(1,5)(2,6)(3,7)])$, $\operatorname{Group}([(1,7)(2,4)(3,5)(6,8),(2,6)(4,8)]), \operatorname{Group}([(2,6)(3,7),(1,4)(2,3)$

$(5,8)(6,7)]), \operatorname{Group}([(2,6)(3,7),(2,6)(3,7),(1,5)]), \operatorname{Group}([(1,5)(2,6)$

$(3,7)(4,8),(1,5)(4,8),(2,6)(3,7)]), \operatorname{Group}([(2,6)(3,7),(2,6)(3,7),(1,5)$ 
$(2,6)]), \operatorname{Group}([(2,6)(3,7),(2,6)(3,7),(1,5)(2,6)(4,8)]), \operatorname{Group}([(1,8)$ $(2,3)(4,5)(6,7),(2,6)(3,7)]), \operatorname{Group}([(1,5)(2,6)(3,7)(4,8),(1,5)(2,6)$ $(3,7)(4,8),(1,5)]), \operatorname{Group}([(1,5)(4,8),(1,5)(4,8),(2,6)(3,7)(4,8)])$, $\operatorname{Group}([(1,5)(3,7),(1,5)(3,7),(2,6)(3,7)(4,8)]), \operatorname{Group}([(1,5)$ $(2,6),(1,5)(2,6),(2,6)(3,7)(4,8)]), \operatorname{Group}([(1,2)(3,4)(5,6)(7,8),(1,5)$

$(2,6),(1,5)(2,6)]), \operatorname{Group}([(1,6)(2,5)(3,8)(4,7),(1,5)(2,6)(3,7)$

$(4,8),(1,5)(2,6)(3,7)(4,8)]), \operatorname{Group}([(1,6)(2,5)(3,8)(4,7),(1,5)$

$(2,6),(1,5)(2,6)]), \operatorname{Group}([(1,2)(3,8)(4,7)(5,6),(1,5)(2,6)(3,7)$

$(4,8),(1,5)(2,6)(3,7)(4,8)]), \operatorname{Group}([(1,3)(2,4)(5,7)(6,8),(1,5)$

$(3,7),(1,5)(3,7)]), \operatorname{Group}([(1,3)(2,4)(5,7)(6,8),(1,5)(2,6)(3,7)$

$(4,8),(1,5)(2,6)(3,7)(4,8)]), \operatorname{Group}([(1,3)(2,8)(4,6)(5,7),(1,5)$

$(3,7),(1,5)(3,7)]), \operatorname{Group}([(1,3)(2,8)(4,6)(5,7),(1,5)(2,6)(3,7)$

$(4,8),(1,5)(2,6)(3,7)(4,8)]), \operatorname{Group}([(1,8)(2,3)(4,5)(6,7),(1,5)$

$(4,8)]), \operatorname{Group}([(1,8)(2,7)(3,6)(4,5),(1,5)(2,6)(3,7)(4,8)])$,

$\operatorname{Group}([(1,5)(4,8),(1,4)(2,7)(3,6)(5,8),(1,5)(4,8)]), \operatorname{Group}([(1,5)(2,6)$

$(3,7)(4,8),(1,4)(2,7)(3,6)(5,8),(1,5)(2,6)(3,7)(4,8)])]$

gap> Length (GsHNPfalseC2xC2);

53

gap> GsHNPtrueC2xC2:=Filtered(GsHNPtrue, $\mathrm{x}->\operatorname{IdSmallGroup}(\mathrm{x})=[4,2])$;

$[\operatorname{Group}([(1,3)(2,4)(5,7)(6,8),(),(1,4)(2,3)(5,8)(6,7)]), \operatorname{Group}([(1,7)$

$(2,8)(3,5)(4,6),(),(1,8)(2,7)(3,6)(4,5)]), \operatorname{Group}([(1,3)(2,8)(4,6)$

$(5,7),(),(1,8)(2,3)(4,5)(6,7)]), \operatorname{Group}([(1,7)(2,4)(3,5)(6,8),(),(1,4)$

$(2,7)(3,6)(5,8)]), \operatorname{Group}([(1,3)(2,4)(5,7)(6,8),(),(1,8)(2,7)(3,6)$

$(4,5)]), \operatorname{Group}([(1,3)(2,8)(4,6)(5,7),(),(1,4)(2,7)(3,6)(5,8)])$,

$\operatorname{Group}([(1,7)(2,8)(3,5)(4,6),(),(1,4)(2,3)(5,8)(6,7)]), \operatorname{Group}([(1,7)$

$(2,4)(3,5)(6,8),(),(1,8)(2,3)(4,5)(6,7)])]$

gap> Length (GsHNPtrueC2xC2);

8

gap> Collected(List (GsHNPfalseC2xC2, $\mathrm{x}->\operatorname{List}(\operatorname{Orbits}(\mathrm{x})$, Length)));

$[[[2,2], 6],[[2,2,2], 16],[[2,2,2,2], 13]$,

$[[2,2,4], 1],[[2,4,2], 5],[[4,2,2], 6],[[4,4], 6]]$ gap> Collected(List (GsHNPtrueC2xC2, $\mathrm{x}->$ List (Orbits (x), Length)));

[ [ [ 4,4 ], 8] ]

gap> GsHNPfalse44C2xC2:=Filtered(GsHNPfalseC2xC2,

$>\mathrm{x}->\operatorname{List}(\operatorname{Orbits}(\mathrm{x},[1 \ldots 8])$, Length) $=[4,4])$;

$[\operatorname{Group}([(1,6)(2,5)(3,8)(4,7),(1,5)(2,6)(3,7)(4,8),(1,5)(2,6)(3,7)$

$(4,8)]), \operatorname{Group}([(1,2)(3,8)(4,7)(5,6),(1,5)(2,6)(3,7)(4,8),(1,5)(2,6)$ $(3,7)(4,8)]), \operatorname{Group}([(1,3)(2,4)(5,7)(6,8),(1,5)(2,6)(3,7)(4,8),(1,5)$ $(2,6)(3,7)(4,8)]), \operatorname{Group}([(1,3)(2,8)(4,6)(5,7),(1,5)(2,6)(3,7)$

$(4,8),(1,5)(2,6)(3,7)(4,8)]), \operatorname{Group}([(1,8)(2,7)(3,6)(4,5),(1,5)(2,6)$ $(3,7)(4,8)]), \operatorname{Group}([(1,5)(2,6)(3,7)(4,8),(1,4)(2,7)(3,6)(5,8),(1,5)$ $(2,6)(3,7)(4,8)])]$

gap> List (GsHNPtrueC2xC2, Elements) ;

$[[(),(1,2)(3,4)(5,6)(7,8),(1,3)(2,4)(5,7)(6,8),(1,4)(2,3)(5,8)(6,7)]$, $[(),(1,2)(3,4)(5,6)(7,8),(1,7)(2,8)(3,5)(4,6),(1,8)(2,7)(3,6)(4,5)]$, $[(),(1,2)(3,8)(4,7)(5,6),(1,3)(2,8)(4,6)(5,7),(1,8)(2,3)(4,5)(6,7)]$, [ ()$,(1,2)(3,8)(4,7)(5,6),(1,4)(2,7)(3,6)(5,8),(1,7)(2,4)(3,5)(6,8)]$, $[(),(1,3)(2,4)(5,7)(6,8),(1,6)(2,5)(3,8)(4,7),(1,8)(2,7)(3,6)(4,5)]$, $[(),(1,3)(2,8)(4,6)(5,7),(1,4)(2,7)(3,6)(5,8),(1,6)(2,5)(3,4)(7,8)]$, $[(),(1,4)(2,3)(5,8)(6,7),(1,6)(2,5)(3,8)(4,7),(1,7)(2,8)(3,5)(4,6)]$, $[(),(1,6)(2,5)(3,4)(7,8),(1,7)(2,4)(3,5)(6,8),(1,8)(2,3)(4,5)(6,7)]]$ gap> List (GsHNPfalseC2xC2, Elements);

$[[(),(4,8),(3,7),(3,7)(4,8)],[(),(4,8),(2,6),(2,6)(4,8)]$, $[(),(4,8),(2,6)(3,7),(2,6)(3,7)(4,8)],[(),(4,8),(1,5),(1,5)(4,8)]$,

$[(),(4,8),(1,5)(3,7),(1,5)(3,7)(4,8)]$,

$[(),(4,8),(1,5)(2,6),(1,5)(2,6)(4,8)]$,

$[(),(4,8),(1,5)(2,6)(3,7),(1,5)(2,6)(3,7)(4,8)]$,

$[(),(3,7),(2,6),(2,6)(3,7)]$,

$[(),(3,7),(2,6)(4,8),(2,6)(3,7)(4,8)]$,

$[(),(3,7),(1,5),(1,5)(3,7)]$,

$[(),(3,7),(1,5)(4,8),(1,5)(3,7)(4,8)]$,

$[(),(3,7),(1,5)(2,6),(1,5)(2,6)(3,7)]$,

$[(),(3,7),(1,5)(2,6)(4,8),(1,5)(2,6)(3,7)(4,8)]$, 
$[(),(3,7)(4,8),(2,6),(2,6)(3,7)(4,8)]$,

$[(),(3,7)(4,8),(2,6)(4,8),(2,6)(3,7)]$,

$[(),(3,7)(4,8),(1,2)(3,4)(5,6)(7,8),(1,2)(3,8)(4,7)(5,6)]$,

$[(),(3,7)(4,8),(1,5),(1,5)(3,7)(4,8)]$,

$[(),(3,7)(4,8),(1,5)(4,8),(1,5)(3,7)]$,

$[(),(3,7)(4,8),(1,5)(2,6),(1,5)(2,6)(3,7)(4,8)]$,

$[(),(3,7)(4,8),(1,5)(2,6)(4,8),(1,5)(2,6)(3,7)]$,

$[(),(3,7)(4,8),(1,6)(2,5)(3,4)(7,8),(1,6)(2,5)(3,8)(4,7)]$,

$[(),(2,6),(1,5),(1,5)(2,6)]$,

$[(),(2,6),(1,5)(4,8),(1,5)(2,6)(4,8)]$,

$[(),(2,6),(1,5)(3,7),(1,5)(2,6)(3,7)]$,

$[(),(2,6),(1,5)(3,7)(4,8),(1,5)(2,6)(3,7)(4,8)]$,

$[(),(2,6)(4,8),(1,3)(2,4)(5,7)(6,8),(1,3)(2,8)(4,6)(5,7)]$,

$[(),(2,6)(4,8),(1,5),(1,5)(2,6)(4,8)]$,

$[(),(2,6)(4,8),(1,5)(4,8),(1,5)(2,6)]$,

$[(),(2,6)(4,8),(1,5)(3,7),(1,5)(2,6)(3,7)(4,8)]$,

$[(),(2,6)(4,8),(1,5)(3,7)(4,8),(1,5)(2,6)(3,7)]$,

$[(),(2,6)(4,8),(1,7)(2,4)(3,5)(6,8),(1,7)(2,8)(3,5)(4,6)]$,

$[(),(2,6)(3,7),(1,4)(2,3)(5,8)(6,7),(1,4)(2,7)(3,6)(5,8)]$,

$[(),(2,6)(3,7),(1,5),(1,5)(2,6)(3,7)]$,

$[(),(2,6)(3,7),(1,5)(4,8),(1,5)(2,6)(3,7)(4,8)]$,

$[(),(2,6)(3,7),(1,5)(3,7),(1,5)(2,6)]$,

$[(),(2,6)(3,7),(1,5)(3,7)(4,8),(1,5)(2,6)(4,8)]$,

$[(),(2,6)(3,7),(1,8)(2,3)(4,5)(6,7),(1,8)(2,7)(3,6)(4,5)]$,

$[(),(2,6)(3,7)(4,8),(1,5),(1,5)(2,6)(3,7)(4,8)]$,

$[(),(2,6)(3,7)(4,8),(1,5)(4,8),(1,5)(2,6)(3,7)]$,

$[(),(2,6)(3,7)(4,8),(1,5)(3,7),(1,5)(2,6)(4,8)]$,

$[(),(2,6)(3,7)(4,8),(1,5)(3,7)(4,8),(1,5)(2,6)]$,

$[(),(1,2)(3,4)(5,6)(7,8),(1,5)(2,6),(1,6)(2,5)(3,4)(7,8)]$,

$[(),(1,2)(3,4)(5,6)(7,8),(1,5)(2,6)(3,7)(4,8),(1,6)(2,5)(3,8)(4,7)]$,

$[(),(1,2)(3,8)(4,7)(5,6),(1,5)(2,6),(1,6)(2,5)(3,8)(4,7)]$,

$[(),(1,2)(3,8)(4,7)(5,6),(1,5)(2,6)(3,7)(4,8),(1,6)(2,5)(3,4)(7,8)]$,

$[(),(1,3)(2,4)(5,7)(6,8),(1,5)(3,7),(1,7)(2,4)(3,5)(6,8)]$,

$[(),(1,3)(2,4)(5,7)(6,8),(1,5)(2,6)(3,7)(4,8),(1,7)(2,8)(3,5)(4,6)]$,

$[(),(1,3)(2,8)(4,6)(5,7),(1,5)(3,7),(1,7)(2,8)(3,5)(4,6)]$,

$[(),(1,3)(2,8)(4,6)(5,7),(1,5)(2,6)(3,7)(4,8),(1,7)(2,4)(3,5)(6,8)]$,

$[(),(1,4)(2,3)(5,8)(6,7),(1,5)(4,8),(1,8)(2,3)(4,5)(6,7)]$,

$[(),(1,4)(2,3)(5,8)(6,7),(1,5)(2,6)(3,7)(4,8),(1,8)(2,7)(3,6)(4,5)]$,

$[(),(1,4)(2,7)(3,6)(5,8),(1,5)(4,8),(1,8)(2,7)(3,6)(4,5)]$,

$[(),(1,4)(2,7)(3,6)(5,8),(1,5)(2,6)(3,7)(4,8),(1,8)(2,3)(4,5)(6,7)]]$ gap> List (GsHNPfalse44C2xC2, Elements);

$[[(),(1,2)(3,4)(5,6)(7,8),(1,5)(2,6)(3,7)(4,8),(1,6)(2,5)(3,8)(4,7)]$,

$[(),(1,2)(3,8)(4,7)(5,6),(1,5)(2,6)(3,7)(4,8),(1,6)(2,5)(3,4)(7,8)]$,

$[(),(1,3)(2,4)(5,7)(6,8),(1,5)(2,6)(3,7)(4,8),(1,7)(2,8)(3,5)(4,6)]$,

$[(),(1,3)(2,8)(4,6)(5,7),(1,5)(2,6)(3,7)(4,8),(1,7)(2,4)(3,5)(6,8)]$,

$[(),(1,4)(2,3)(5,8)(6,7),(1,5)(2,6)(3,7)(4,8),(1,8)(2,7)(3,6)(4,5)]$,

$[(),(1,4)(2,7)(3,6)(5,8),(1,5)(2,6)(3,7)(4,8),(1,8)(2,3)(4,5)(6,7)]]$ gap> ZG: $=$ Centre $(G)$;

$\operatorname{Group}([(1,5)(2,6)(3,7)(4,8)])$

gap> List (GsHNPtrueC2xC2, $\mathrm{x}->$ Intersection $(\mathrm{x}, \mathrm{ZG}))$;

[ $\operatorname{Group}(()), \operatorname{Group}(()), \operatorname{Group}(()), \operatorname{Group}(()), \operatorname{Group}(()), \operatorname{Group}(())$,

$\operatorname{Group}(()), \operatorname{Group}(())]$

gap> List (GsHNPfalse44C2xC2, $\mathrm{x}->$ Intersection $(\mathrm{x}, \mathrm{ZG})$ ) ;

$[\operatorname{Group}([(1,5)(2,6)(3,7)(4,8)]), \operatorname{Group}([(1,5)(2,6)(3,7)(4,8)])$,

$\operatorname{Group}([(1,5)(2,6)(3,7)(4,8)]), \operatorname{Group}([(1,5)(2,6)(3,7)(4,8)])$,

$\operatorname{Group}([(1,5)(2,6)(3,7)(4,8)]), \operatorname{Group}([(1,5)(2,6)(3,7)(4,8)])]$

gap> DG:=DerivedSubgroup $(G)$;

Group $([(1,5)(4,8),(2,6)(4,8),(3,7)(4,8)])$

gap> StructureDescription(DG);

"C2 x C2 x C2"

gap> Collected(List (GsHNPfalseC2xC2, x->Order (Intersection(DG, x)))) ;

[ [ 2,46], [4,7] ]

gap> Collected (List (GsHNPtrueC2xC2, x->0rder (Intersection(DG, x)))) ; 
[ $[1,8]]$

gap> GsHNPfalseC4xC2:=Filtered (GsHNPfalse, $x->\operatorname{IdSmallGroup}(\mathrm{x})=[8,2]$ ) ;

$[\operatorname{Group}([(1,2)(3,4,7,8)(5,6),(1,5)(2,6)(3,7)(4,8),(1,5)(2,6)(3,7)$

$(4,8),(1,5)(2,6)]), \operatorname{Group}([(1,2,5,6)(3,8)(4,7),(1,5)(2,6)(3,7)$

$(4,8),(1,5)(2,6)(3,7)(4,8),(3,7)(4,8)]), \operatorname{Group}([(1,2,5,6)$

$(3,8,7,4),(1,5)(2,6)(3,7)(4,8),(1,5)(2,6)(3,7)(4,8),(3,7)(4,8)])$,

$\operatorname{Group}([(1,3)(2,8,6,4)(5,7),(1,5)(2,6)(3,7)(4,8),(1,5)(2,6)(3,7)$

$(4,8),(2,6)(4,8)]), \operatorname{Group}([(1,3,5,7)(2,4)(6,8),(1,5)(2,6)(3,7)$

$(4,8),(1,5)(2,6)(3,7)(4,8),(1,5)(3,7)]), \operatorname{Group}([(1,7,5,3)$

$(2,8,6,4),(1,5)(2,6)(3,7)(4,8),(1,5)(2,6)(3,7)(4,8),(2,6)(4,8)])$,

Group $([(1,5)(2,6)(3,7)(4,8),(1,8,5,4)(2,3)(6,7),(1,5)(4,8),(2,6)$

$(3,7)]), \operatorname{Group}([(1,4)(2,7,6,3)(5,8),(1,5)(4,8),(2,6)(3,7)])$,

$\operatorname{Group}([(1,5)(4,8),(1,8,5,4)(2,3,6,7),(1,5)(2,6)(3,7)(4,8)])]$ gap> Length (GsHNPfalseC4xC2);

9

gap> GsHNPtrueC4xC2:=Filtered (GsHNPtrue, $\mathrm{x}->\operatorname{IdSmallGroup}(\mathrm{x})=[8,2]$ ) ;

$[\operatorname{Group}([(1,7,5,3)(2,8,6,4),(),(1,8,5,4)(2,7,6,3),(1,5)(2,6)(3,7)$

$(4,8)]), \operatorname{Group}([(1,7,5,3)(2,4,6,8),(),(1,8,5,4)(2,3,6,7),(1,5)(2,6)$

$(3,7)(4,8)]), \operatorname{Group}([(1,3)(2,4)(5,7)(6,8),(),(1,8,5,4)(2,3,6,7),(1,5)$

$(2,6)(3,7)(4,8)]), \operatorname{Group}([(1,7,5,3)(2,8,6,4),(),(1,4)(2,7)(3,6)$

$(5,8),(1,5)(2,6)(3,7)(4,8)]), \operatorname{Group}([(1,3)(2,8)(4,6)(5,7),(),(1,8,5,4)$

$(2,7,6,3),(1,5)(2,6)(3,7)(4,8)]), \operatorname{Group}([(1,7,5,3)(2,4,6,8),(),(1,4)$

$(2,3)(5,8)(6,7),(1,5)(2,6)(3,7)(4,8)])]$

gap> Length (GsHNPtrueC4xC2);

6

gap> Collected(List (GsHNPfalseC4xC2, $\mathrm{x}->\operatorname{List}(\operatorname{Orbits}(\mathrm{x})$, Length)));

[ [ [ 4,4 ], 9$]]$

gap> Collected (List (GsHNPtrueC4xC2, $\mathrm{x}->$ List (Orbits (x), Length))) ;

[ [ [ 8$], 6]]$

gap> Collected (List (GsHNPfalseC4xC2, $\mathrm{x}->\operatorname{Order}(\operatorname{Intersection}(\mathrm{DG}, \mathrm{x})))$ ))

$[4,9]]$

gap> Collected (List (GsHNPtrueC4xC2, x->Order (Intersection(DG, x)))) ;

$[[2,6]]$

$$
(1-4-2) G=8 T 38 \simeq\left(\left(\left(C_{2}\right)^{4} \rtimes C_{2}\right) \rtimes C_{2}\right) \rtimes C_{3} .
$$

gap> Read("HNP.gap") ;

gap> G:=TransitiveGroup $(8,38) ; \# \mathrm{G}=8 \mathrm{~T} 38=((\mathrm{C} 2 \sim 4: \mathrm{C} 2): \mathrm{C} 2): \mathrm{C} 3$

$[2 \sim 4] \mathrm{A}(4)$

gap> GeneratorsOfGroup $(G)$;

$[(4,8),(1,8)(2,3)(4,5)(6,7),(1,2,3)(5,6,7)]$

gap> $\mathrm{H}:=$ Stabilizer $(\mathrm{G}, 1)$; \# $\mathrm{H}=\mathrm{C} 2 \times \mathrm{xA} 4$

$\operatorname{Group}([(4,8),(2,6),(2,8,3)(4,7,6)])$

gap> FirstObstructionN(G).ker; \# Obs1N=1

[ [ ], [ [ 6 ], [ ] ] ]

gap> SchurMultPcpGroup $(G) ; \# M(G)=C 2 \times C 2$ : Schur multiplier of $G$

$[2,2]$

gap> cGs:=MinimalStemExtensions $(G) ; ; \# 3$ minimal stem extensions

gap> for $c G$ in cGs do

$>\mathrm{bG}:=\mathrm{cG}$.MinimalStemExtension;

$>$ bH: $=$ PreImage (cG.epi, H) ;

$>\operatorname{Print}(\operatorname{KerResH3Z}(\mathrm{bG}, \mathrm{bH}))$;

$>$ Print("\n");

$>$ od;

[ [ 2$],[[2],[[1]]]]$

$[[],[[2,2],[]]]$

[ [ 2$],[[2],[[1]]]]$

gap> for $\mathrm{CG}$ in cGs do

$>\mathrm{bG}:=\mathrm{cG}$.MinimalStemExtension;

$>\mathrm{bH}:=\operatorname{PreImage}(\mathrm{cG}$. epi, $\mathrm{H})$;

$>$ Print (FirstObstructionN (bG,bH). ker [1]);

$>$ Print (FirstObstructionDnr (bG,bH).Dnr [1]);

$>\operatorname{Print}(" \backslash \mathrm{n} ")$;

$>$ od; 
$[$ [ ] ]

$[2][]$

$[$ [ ] ]

gap> cG:=cGs [2]; ;

gap> bG:=cG.MinimalStemExtension; \# bG=G- is a minimal stem extension of $G$ <permutation group of size 384 with 8 generators>

gap> bH:=PreImage (cG.epi,H); \# bH=H-

<permutation group of size 48 with 3 generators>

gap> FirstObstructionN (bG,bH).ker; \# Obs1N-=C2

[ [ 2$],[[2,6],[[1,0]]]]$

gap> FirstObstructionDnr(bG,bH).Dnr; \# Obs1Dnr-=1

$[$ [ ], [ [ 2,6 ], [ ] ] ]

gap> bGs: $=$ AllSubgroups $(b G) ;$;

gap> Length(bGs);

1002

gap> bGsHNPfalse:=Filtered(bGs, x->FirstObstructionDr (bG, x, bH).Dr [1] =[] ); ;

gap> Length(bGsHNPfalse);

951

gap> bGsHNPtrue:=Filtered(bGs, $\mathrm{x}->$ FirstObstructionDr (bG , x, bH).Dr [1] =[2]) ; ;

gap> Length(bGsHNPtrue);

51

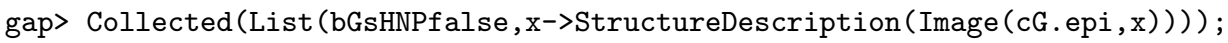

[ [ "(C2 x C2 x C2 x C2) : C2", 3 ], [ "(C4 x C2) : C2", 45 ], [ "1", 2 ],

[ "A4", 8 ], [ "C2", 57 ], [ "C2 x A4", 36 ], [ "C2 x C2", 193 ],

[ "C2 x C2 x A4", 20 ], [ "C2 x C2 x C2", 138],

[ "C2 $x$ C2 2 x $\mathrm{C} 2 \times \mathrm{C} 2$ ", 17 ], [ "C2 $x$ D8", 9 ], [ "C3", 32 ], [ "C4", 54 ],

[ "C4 x C2", 45 ], [ "C6", 144 ], [ "C6 x C2", 80 ], [ "D8", 42 ],

[ "Q8", 10$],[$ "SL $(2,3) ", 16$ ] ]

gap> Collected (List (bGsHNPtrue, $\mathrm{x}->$ StructureDescription (Image (cG.epi, $\mathrm{x}))$ )) ;

[ [ $"(((\mathrm{C} 2 \times \mathrm{C} 2 \times \mathrm{C} 2 \times \mathrm{C} 2): \mathrm{C} 2): \mathrm{C} 2): \mathrm{C} 3 ", 1]$,

$["((\mathrm{C} 2 \times \mathrm{C} 2 \times \mathrm{C} 2 \times \mathrm{C} 2): \mathrm{C} 2): \mathrm{C} 2 ", 1]$,

$["((\mathrm{C} 2 \times \mathrm{C} 2 \times \mathrm{C} 2):(\mathrm{C} 2 \times \mathrm{C} 2)): \mathrm{C} 3 ", 1]$,

[ "(C2 $x$ C2 $\times$ C2 $):(\mathrm{C} 2 \times \mathrm{C} 2) ", 1],["(\mathrm{C} 2 \times \mathrm{C} 2 \times \mathrm{C} 2): \mathrm{C} 4 ", 3]$,

[ "(C4 x C2) : C2", 6 ], [ "A4", 8 ], [ "C2 $\mathrm{x} \mathrm{A4",} 8$ ], [ "C2 x C2", 8 ],

[ "C2 x C2 x C2", 2 ], [ "C2 x D8", 6 ], [ "C4 x C2", 6 ] ]

gap> GsHNPfalse:=Set (bGsHNPfalse, $\mathrm{x}->\operatorname{Image}(\mathrm{cG}$. epi, $\mathrm{x})$ ) ; ;

gap> Length(GsHNPfalse);

300

gap> GsHNPtrue:=Set (bGsHNPtrue, $\mathrm{x}->\operatorname{Image}(\mathrm{cG}$. epi, $\mathrm{x})$ ) ; ;

gap> Length(GsHNPtrue);

51

gap> Intersection(GsHNPfalse, GsHNPtrue);

[ ]

gap> GsHNPtrueMin:=Filtered (GsHNPtrue, $\mathrm{x}->$ Length (Filtered (GsHNPtrue,

$>\mathrm{y}->\operatorname{IsSubgroup}(\mathrm{x}, \mathrm{y})))=1)$;

[ $\operatorname{Group}([(1,2)(3,4)(5,6)(7,8),(1,3)(2,8,6,4)(5,7),(),(2,6)(4,8),(1,5)$

$(3,7),(1,5)(4,8)]), \operatorname{Group}([(1,3)(2,4)(5,7)(6,8),(1,8,5,4)(2,3)$

$(6,7),(),(1,5)(4,8),(2,6)(3,7),(3,7)(4,8)]), \operatorname{Group}([(1,4)(2,3)(5,8)$

$(6,7),(1,2)(3,8,7,4)(5,6),(),(3,7)(4,8),(1,5)(2,6),(2,6)(4,8)])$,

$\operatorname{Group}([(1,3)(2,4)(5,7)(6,8),(1,4)(2,3)(5,8)(6,7),()]), \operatorname{Group}([(1,2)$

$(3,4)(5,6)(7,8),(1,7,5,3)(2,8,6,4),(),(1,5)(2,6)(3,7)(4,8)])$,

$\operatorname{Group}([(1,7)(2,8)(3,5)(4,6),(1,8)(2,7)(3,6)(4,5),()]), \operatorname{Group}([(1,3)$

$(2,8)(4,6)(5,7),(1,8)(2,3)(4,5)(6,7),()]), \operatorname{Group}([(1,2)(3,8)(4,7)$

$(5,6),(1,7,5,3)(2,4,6,8),(),(1,5)(2,6)(3,7)(4,8)]), \operatorname{Group}([(1,7)(2,4)$

$(3,5)(6,8),(1,4)(2,7)(3,6)(5,8),()]), \operatorname{Group}([(1,3)(2,4)(5,7)$

$(6,8),(1,8,5,4)(2,3,6,7),(),(1,5)(2,6)(3,7)(4,8)]), \operatorname{Group}([(1,8)(2,3)$

$(4,5)(6,7),(1,2,5,6)(3,4,7,8),(),(1,5)(2,6)(3,7)(4,8)]), \operatorname{Group}([(1,3)$

$(2,8)(4,6)(5,7),(1,4,5,8)(2,3,6,7),(),(1,5)(2,6)(3,7)(4,8)])$,

$\operatorname{Group}([(1,4)(2,3)(5,8)(6,7),(1,2,5,6)(3,8,7,4),(),(1,5)(2,6)(3,7)$

$(4,8)]), \operatorname{Group}([(1,3)(2,4)(5,7)(6,8),(1,8)(2,7)(3,6)(4,5),()])$,

$\operatorname{Group}([(1,3)(2,8)(4,6)(5,7),(1,4)(2,7)(3,6)(5,8),()]), \operatorname{Group}([(1,6)$

$(2,5)(3,8)(4,7),(1,7)(2,8)(3,5)(4,6),()]), \operatorname{Group}([(1,6)(2,5)(3,4)$

$(7,8),(1,7)(2,4)(3,5)(6,8),()])]$ 
gap> Length(GsHNPtrueMin);

17

gap> List (GsHNPtrueMin, IdSmallGroup);

$[[32,6],[32,6],[32,6],[4,2],[8,2],[4,2],[4,2]$,

$[8,2],[4,2],[8,2],[8,2],[8,2],[8,2],[4,2]$,

$[4,2],[4,2],[4,2]]$

gap $>$ Collected (List (GsHNPfalse, $\mathrm{x}->$ Filtered (GsHNPtrueMin, $y->\operatorname{IsSubgroup}(\mathrm{x}, \mathrm{y})$ ))) ;

$[[[], 300]]$

gap> Gs:=AllSubgroups $(G)$; ;

gap> Length(Gs);

351

gap> GsC2xC2:=Filtered(Gs, $\mathrm{x}->\operatorname{IdSmallGroup}(\mathrm{x})=[4,2]) ;$;

gap> Length (GsC2xC2);

61

gap> GsC4xC2:=Filtered(Gs,x->IdSmallGroup $(\mathrm{x})=[8,2]) ;$;

gap> Length (GsC4xC2);

15

gap> Gs32_6:=Filtered (Gs, $x->\operatorname{IdSmallGroup~}(\mathrm{x})=[32,6])$; ;

gap> Length(Gs32_6);

3

gap> GsHNPfalseC2xC2: $=$ Filtered (GsHNPfalse, $x->\operatorname{IdSmallGroup}(\mathrm{x})=[4,2])$;

$[\operatorname{Group}([(3,7),(4,8)]), \operatorname{Group}([(2,6),(4,8)]), \operatorname{Group}([(),(4,8),(2,6)$

$(3,7)]), \operatorname{Group}([(4,8),(1,5)(4,8)]), \operatorname{Group}([(4,8),(1,5)(3,7)])$,

$\operatorname{Group}([(1,5)(2,6),(4,8)]), \operatorname{Group}([(4,8),(1,5)(2,6)(3,7)(4,8)])$,

$\operatorname{Group}([(),(2,6),(3,7)]), \operatorname{Group}([(),(3,7),(2,6)(4,8)])$,

$\operatorname{Group}([(),(1,5),(3,7)]), \operatorname{Group}([(3,7),(1,5)(4,8)])$,

$\operatorname{Group}([(3,7),(1,5)(2,6)]), \operatorname{Group}([(),(3,7),(1,5)(2,6)(4,8)])$,

$\operatorname{Group}([(),(2,6),(3,7)(4,8)]), \operatorname{Group}([(),(2,6)(4,8),(3,7)(4,8)])$,

$\operatorname{Group}([(1,2)(3,4)(5,6)(7,8),(),(3,7)(4,8)]), \operatorname{Group}([(3,7)$

$(4,8),(1,5)]), \operatorname{Group}([(3,7)(4,8),(1,5)(4,8)]), \operatorname{Group}([(),(3,7)$

$(4,8),(1,5)(2,6)]), \operatorname{Group}([(1,5)(2,6)(4,8),(3,7)(4,8)]), \operatorname{Group}([(1,6)$

$(2,5)(3,8)(4,7),(),(3,7)(4,8)]), \operatorname{Group}([(),(1,5),(2,6)])$,

$\operatorname{Group}([(1,5)(4,8),(2,6)]), \operatorname{Group}([(2,6),(1,5)(3,7)])$,

$\operatorname{Group}([(),(2,6),(1,5)(3,7)(4,8)]), \operatorname{Group}([(1,3)(2,4)(5,7)$

$(6,8),(),(2,6)(4,8)]), \operatorname{Group}([(),(1,5),(2,6)(4,8)])$,

$\operatorname{Group}([(),(2,6)(4,8),(1,5)(2,6)]), \operatorname{Group}([(),(2,6)(4,8),(1,5)$

$(3,7)]), \operatorname{Group}([(2,6)(4,8),(1,5)(2,6)(3,7)]), \operatorname{Group}([(1,7)(2,8)(3,5)$

$(4,6),(),(2,6)(4,8)]), \operatorname{Group}([(1,4)(2,3)(5,8)(6,7),(),(2,6)(3,7)])$,

$\operatorname{Group}([(),(1,5),(2,6)(3,7)]), \operatorname{Group}([(),(1,5)(4,8),(2,6)(3,7)])$,

$\operatorname{Group}([(),(1,5)(2,6),(2,6)(3,7)]), \operatorname{Group}([(1,5)(3,7)(4,8),(2,6)$

$(3,7)]), \operatorname{Group}([(1,8)(2,3)(4,5)(6,7),(),(2,6)(3,7)]), \operatorname{Group}([(2,6)$

$(3,7)(4,8),(1,5)]), \operatorname{Group}([(),(1,5)(4,8),(2,6)(3,7)(4,8)])$,

Group $([(),(1,5)(3,7),(2,6)(3,7)(4,8)]), \operatorname{Group}([(),(1,5)(2,6),(2,6)$

$(3,7)(4,8)]), \operatorname{Group}([(1,2)(3,4)(5,6)(7,8),(),(1,5)(2,6)])$,

$\operatorname{Group}([(1,2)(3,4)(5,6)(7,8),(),(1,5)(2,6)(3,7)(4,8)]), \operatorname{Group}([(1,2)$

$(3,8)(4,7)(5,6),(),(1,5)(2,6)]), \operatorname{Group}([(1,2)(3,8)(4,7)(5,6),(),(1,5)$

$(2,6)(3,7)(4,8)]), \operatorname{Group}([(1,3)(2,4)(5,7)(6,8),(),(1,5)(3,7)])$,

$\operatorname{Group}([(1,3)(2,4)(5,7)(6,8),(),(1,5)(2,6)(3,7)(4,8)]), \operatorname{Group}([(1,3)$

$(2,8)(4,6)(5,7),(),(1,5)(3,7)]), \operatorname{Group}([(1,3)(2,8)(4,6)(5,7),(),(1,5)$

$(2,6)(3,7)(4,8)]), \operatorname{Group}([(1,4)(2,3)(5,8)(6,7),(),(1,5)(4,8)])$,

$\operatorname{Group}([(1,4)(2,3)(5,8)(6,7),(),(1,5)(2,6)(3,7)(4,8)]), \operatorname{Group}([(1,8)$

$(2,7)(3,6)(4,5),(),(1,5)(4,8)]), \operatorname{Group}([(1,8)(2,3)(4,5)(6,7),(),(1,5)$

$(2,6)(3,7)(4,8)]$ ] ]

gap> Length(GsHNPfalseC2xC2);

53

gap> GsHNPtrueC2xC2:=Filtered(GsHNPtrue, $\mathrm{x}->\operatorname{IdSmallGroup}(\mathrm{x})=[4,2]$ ) ;

$[\operatorname{Group}([(1,3)(2,4)(5,7)(6,8),(1,4)(2,3)(5,8)(6,7),()]), \operatorname{Group}([(1,7)$

$(2,8)(3,5)(4,6),(1,8)(2,7)(3,6)(4,5),()]), \operatorname{Group}([(1,3)(2,8)(4,6)$

$(5,7),(1,8)(2,3)(4,5)(6,7),()]), \operatorname{Group}([(1,7)(2,4)(3,5)(6,8),(1,4)(2,7)$

$(3,6)(5,8),()]), \operatorname{Group}([(1,3)(2,4)(5,7)(6,8),(1,8)(2,7)(3,6)$

$(4,5),()]), \operatorname{Group}([(1,3)(2,8)(4,6)(5,7),(1,4)(2,7)(3,6)(5,8),()])$, $\operatorname{Group}([(1,6)(2,5)(3,8)(4,7),(1,7)(2,8)(3,5)(4,6),()]), \operatorname{Group}([(1,6)$

$(2,5)(3,4)(7,8),(1,7)(2,4)(3,5)(6,8),()])]$ 
gap> Length(GsHNPtrueC2xC2);

8

gap> Collected(List (GsHNPfalseC2xC2, x->List (Orbits (x), Length))) ;

$[[[2,2], 6],[[2,2,2], 16],[[2,2,2,2], 13]$,

$[[2,2,4], 1],[[2,4,2], 5],[[4,2,2], 6],[[4,4], 6]]$ gap> Collected (List (GsHNPtrueC2xC2, $\mathrm{x}->\operatorname{List}(\operatorname{Orbits}(\mathrm{x})$, Length)));

$[[[4,4], 8]]$

gap> GsHNPfalse44C2xC2:=Filtered(GsHNPfalseC2xC2,

$>\mathrm{x}->\operatorname{List}(\operatorname{Orbits}(\mathrm{x},[1 \ldots 8])$, Length) $=[4,4])$;

$[\operatorname{Group}([(1,2)(3,4)(5,6)(7,8),(),(1,5)(2,6)(3,7)(4,8)]), \operatorname{Group}([(1,2)$

$(3,8)(4,7)(5,6),(),(1,5)(2,6)(3,7)(4,8)]), \operatorname{Group}([(1,3)(2,4)(5,7)$

$(6,8),(),(1,5)(2,6)(3,7)(4,8)]), \operatorname{Group}([(1,3)(2,8)(4,6)(5,7),(),(1,5)$

$(2,6)(3,7)(4,8)]), \operatorname{Group}([(1,4)(2,3)(5,8)(6,7),(),(1,5)(2,6)(3,7)$

$(4,8)]), \operatorname{Group}([(1,8)(2,3)(4,5)(6,7),(),(1,5)(2,6)(3,7)(4,8)])]$

gap> List (GsHNPtrueC2xC2, Elements);

$[[(),(1,2)(3,4)(5,6)(7,8),(1,3)(2,4)(5,7)(6,8),(1,4)(2,3)(5,8)(6,7)]$,

$[(),(1,2)(3,4)(5,6)(7,8),(1,7)(2,8)(3,5)(4,6),(1,8)(2,7)(3,6)(4,5)]$,

$[(),(1,2)(3,8)(4,7)(5,6),(1,3)(2,8)(4,6)(5,7),(1,8)(2,3)(4,5)(6,7)]$,

$[(),(1,2)(3,8)(4,7)(5,6),(1,4)(2,7)(3,6)(5,8),(1,7)(2,4)(3,5)(6,8)]$,

$[(),(1,3)(2,4)(5,7)(6,8),(1,6)(2,5)(3,8)(4,7),(1,8)(2,7)(3,6)(4,5)]$,

$[(),(1,3)(2,8)(4,6)(5,7),(1,4)(2,7)(3,6)(5,8),(1,6)(2,5)(3,4)(7,8)]$,

$[(),(1,4)(2,3)(5,8)(6,7),(1,6)(2,5)(3,8)(4,7),(1,7)(2,8)(3,5)(4,6)]$,

$[(),(1,6)(2,5)(3,4)(7,8),(1,7)(2,4)(3,5)(6,8),(1,8)(2,3)(4,5)(6,7)]]$

gap> List (GsHNPfalseC2xC2,Elements);

$[[(),(4,8),(3,7),(3,7)(4,8)],[(),(4,8),(2,6),(2,6)(4,8)]$,

$[(),(4,8),(2,6)(3,7),(2,6)(3,7)(4,8)],[(),(4,8),(1,5),(1,5)(4,8)]$,

$[(),(4,8),(1,5)(3,7),(1,5)(3,7)(4,8)]$,

$[(),(4,8),(1,5)(2,6),(1,5)(2,6)(4,8)]$,

$[(),(4,8),(1,5)(2,6)(3,7),(1,5)(2,6)(3,7)(4,8)]$,

$[(),(3,7),(2,6),(2,6)(3,7)]$,

$[(),(3,7),(2,6)(4,8),(2,6)(3,7)(4,8)]$,

$[(),(3,7),(1,5),(1,5)(3,7)]$,

$[(),(3,7),(1,5)(4,8),(1,5)(3,7)(4,8)]$,

$[(),(3,7),(1,5)(2,6),(1,5)(2,6)(3,7)]$,

$[(),(3,7),(1,5)(2,6)(4,8),(1,5)(2,6)(3,7)(4,8)]$,

$[(),(3,7)(4,8),(2,6),(2,6)(3,7)(4,8)]$,

$[(),(3,7)(4,8),(2,6)(4,8),(2,6)(3,7)]$,

$[(),(3,7)(4,8),(1,2)(3,4)(5,6)(7,8),(1,2)(3,8)(4,7)(5,6)]$,

$[(),(3,7)(4,8),(1,5),(1,5)(3,7)(4,8)]$,

$[(),(3,7)(4,8),(1,5)(4,8),(1,5)(3,7)]$,

$[(),(3,7)(4,8),(1,5)(2,6),(1,5)(2,6)(3,7)(4,8)]$,

$[(),(3,7)(4,8),(1,5)(2,6)(4,8),(1,5)(2,6)(3,7)]$,

$[(),(3,7)(4,8),(1,6)(2,5)(3,4)(7,8),(1,6)(2,5)(3,8)(4,7)]$,

$[(),(2,6),(1,5),(1,5)(2,6)]$,

$[(),(2,6),(1,5)(4,8),(1,5)(2,6)(4,8)]$,

$[(),(2,6),(1,5)(3,7),(1,5)(2,6)(3,7)]$,

$[(),(2,6),(1,5)(3,7)(4,8),(1,5)(2,6)(3,7)(4,8)]$,

$[(),(2,6)(4,8),(1,3)(2,4)(5,7)(6,8),(1,3)(2,8)(4,6)(5,7)]$,

$[(),(2,6)(4,8),(1,5),(1,5)(2,6)(4,8)]$,

$[(),(2,6)(4,8),(1,5)(4,8),(1,5)(2,6)]$,

$[(),(2,6)(4,8),(1,5)(3,7),(1,5)(2,6)(3,7)(4,8)]$,

$[(),(2,6)(4,8),(1,5)(3,7)(4,8),(1,5)(2,6)(3,7)]$,

$[(),(2,6)(4,8),(1,7)(2,4)(3,5)(6,8),(1,7)(2,8)(3,5)(4,6)]$,

$[(),(2,6)(3,7),(1,4)(2,3)(5,8)(6,7),(1,4)(2,7)(3,6)(5,8)]$,

$[(),(2,6)(3,7),(1,5),(1,5)(2,6)(3,7)]$,

$[(),(2,6)(3,7),(1,5)(4,8),(1,5)(2,6)(3,7)(4,8)]$,

$[(),(2,6)(3,7),(1,5)(3,7),(1,5)(2,6)]$,

$[(),(2,6)(3,7),(1,5)(3,7)(4,8),(1,5)(2,6)(4,8)]$,

$[(),(2,6)(3,7),(1,8)(2,3)(4,5)(6,7),(1,8)(2,7)(3,6)(4,5)]$,

$[(),(2,6)(3,7)(4,8),(1,5),(1,5)(2,6)(3,7)(4,8)]$,

$[(),(2,6)(3,7)(4,8),(1,5)(4,8),(1,5)(2,6)(3,7)]$,

$[(),(2,6)(3,7)(4,8),(1,5)(3,7),(1,5)(2,6)(4,8)]$,

$[(),(2,6)(3,7)(4,8),(1,5)(3,7)(4,8),(1,5)(2,6)]$, 


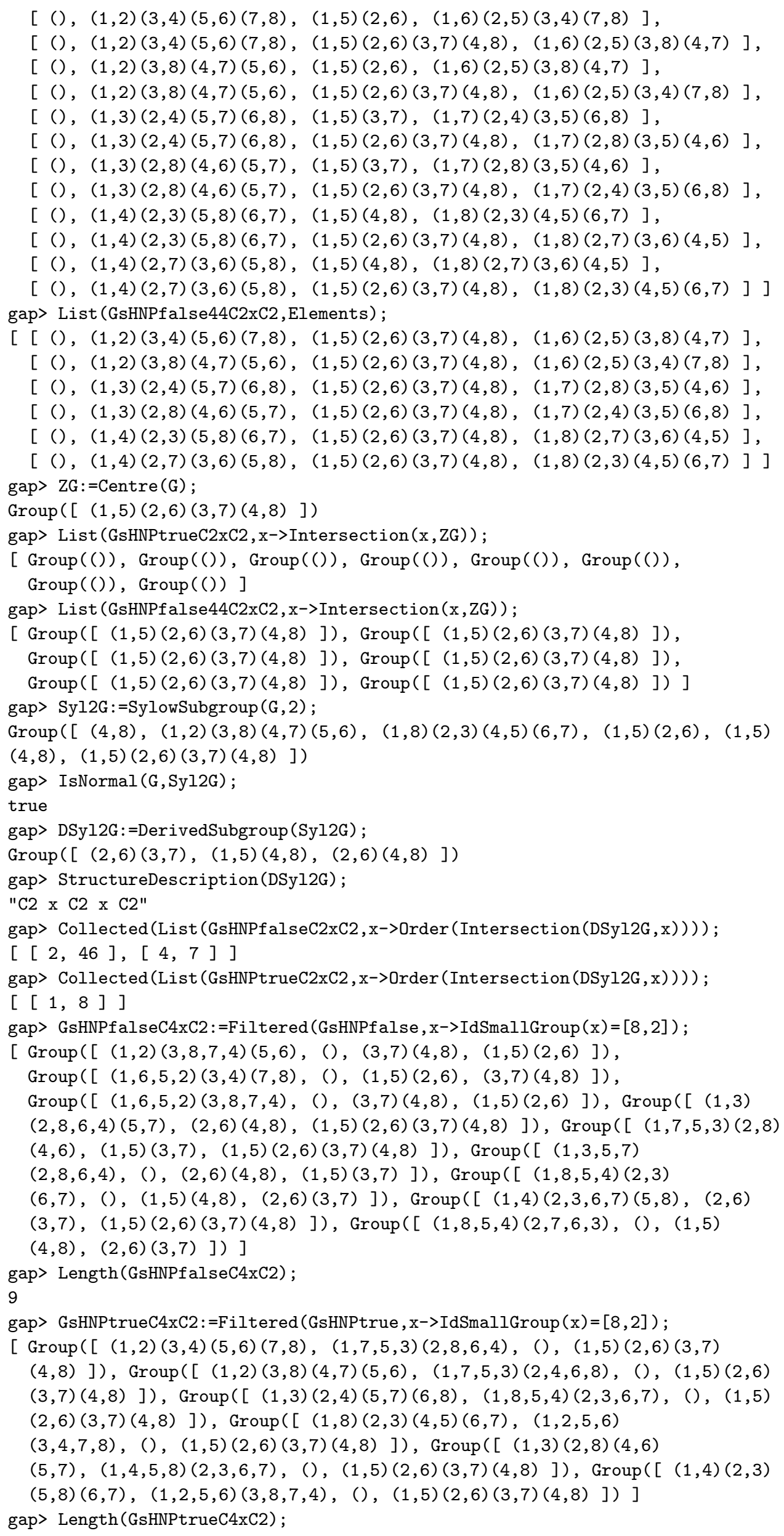




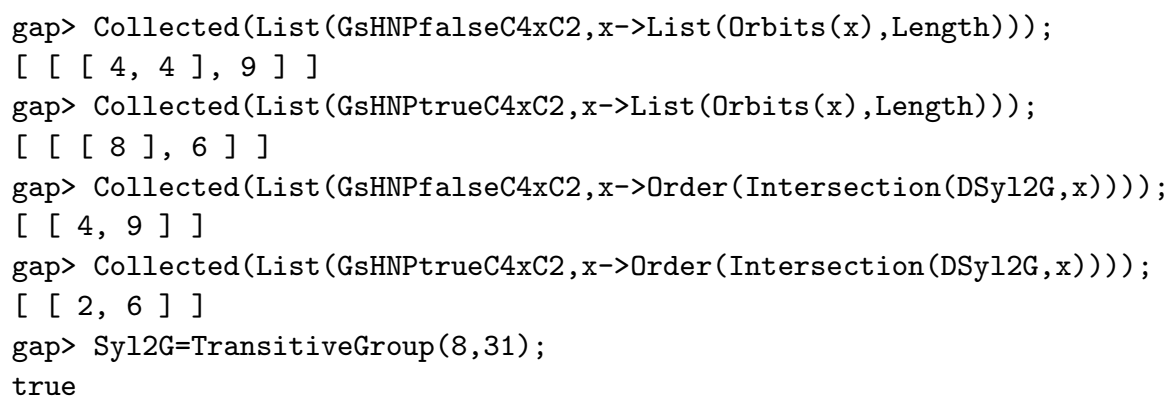

Example 6.12 $(G=8 T m(m=9,11,15,19,22,32))$.

(2-1) $G=8 T 9 \simeq D_{4} \times C_{2}$.

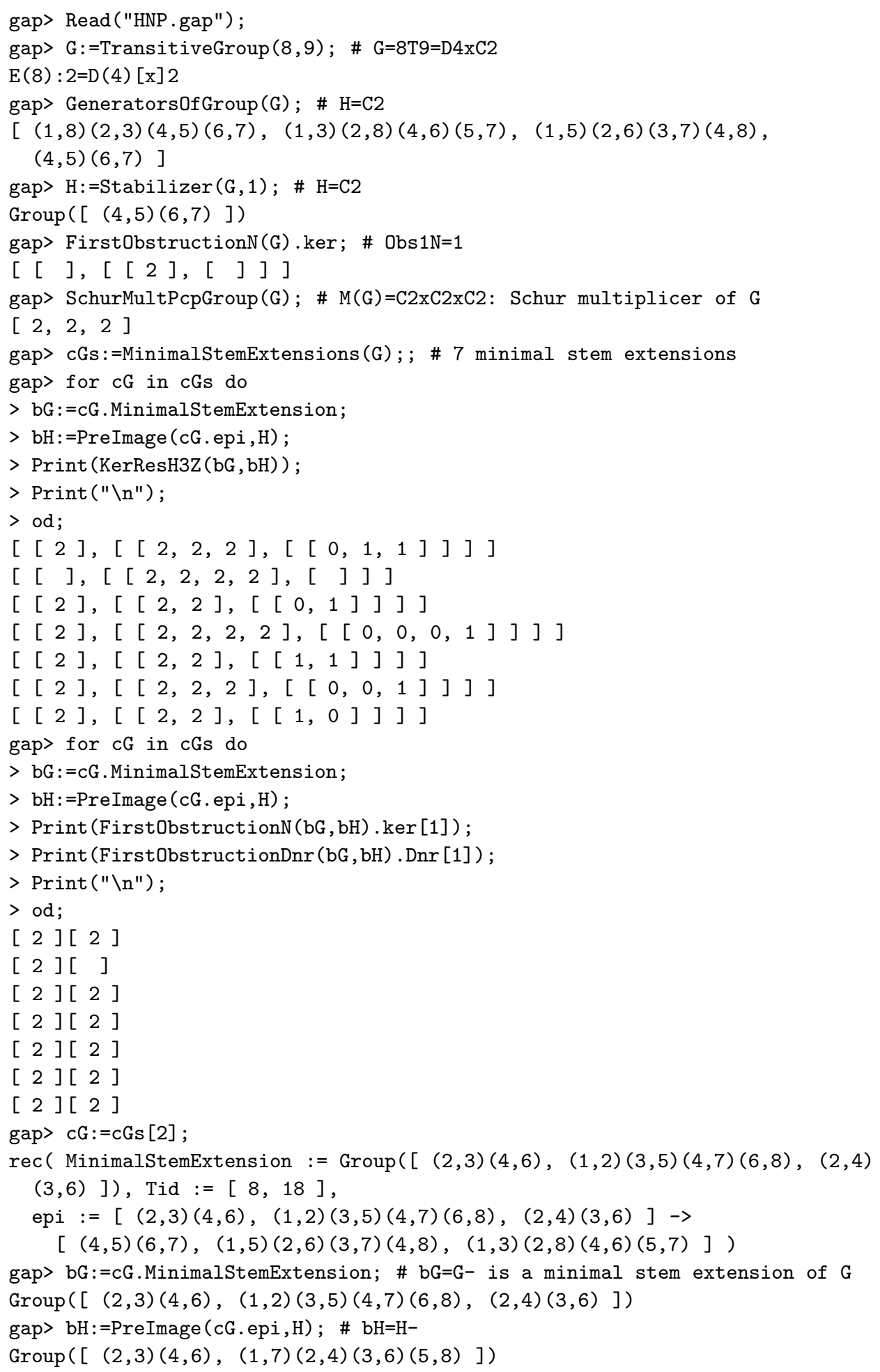




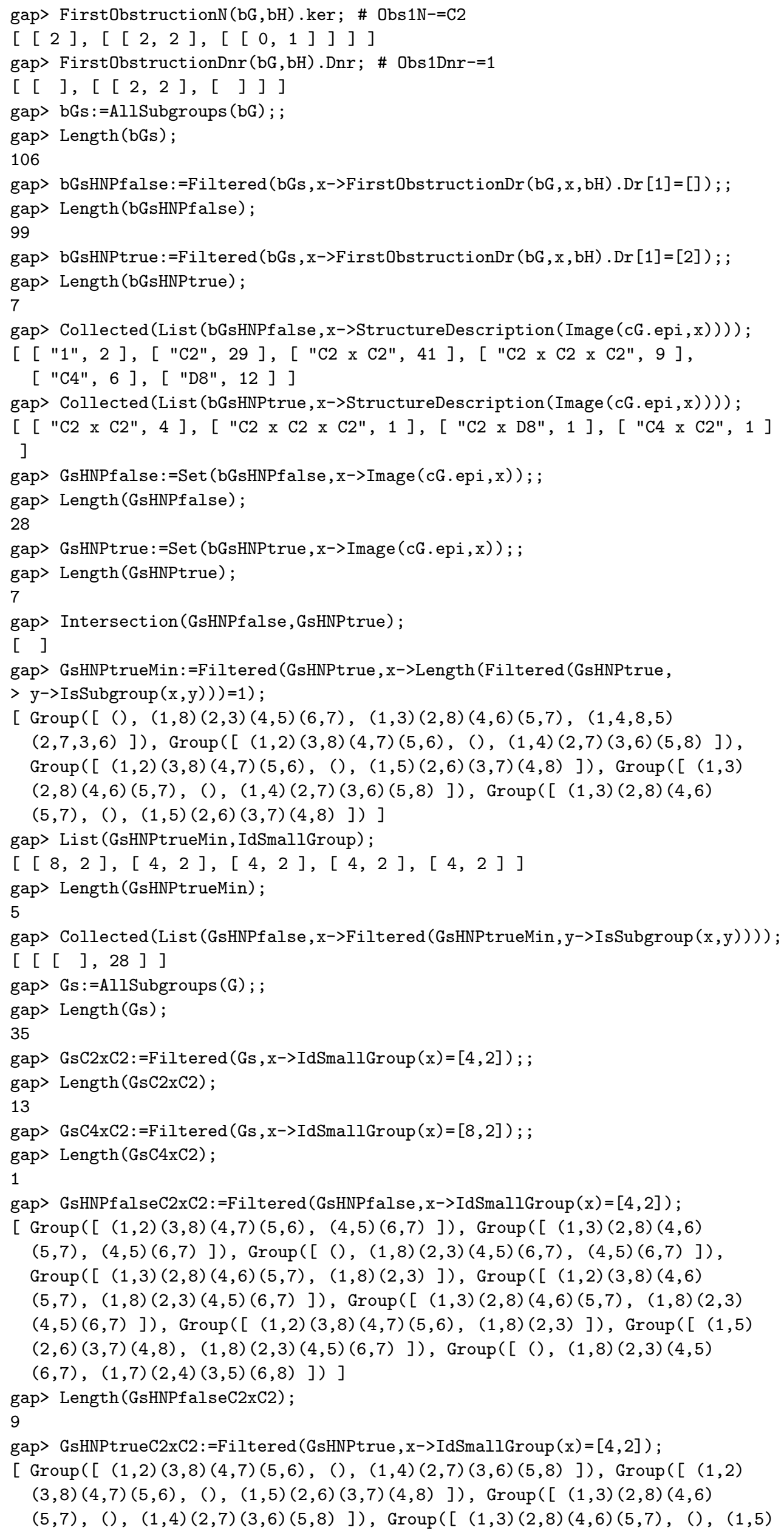


$(2,6)(3,7)(4,8)])]$

gap> Length(GsHNPtrueC2xC2);

4

gap> Collected (List (GsHNPfalseC2xC2, x->List(Orbits (x), Length))) ;

$[[[2,2,2,2], 1],[[2,2,4], 2],[[4,2,2], 2]$,

$[[4,4], 4]]$

gap> Collected(List (GsHNPtrueC2xC2, x->List(Orbits (x), Length)));

$[[[4,4], 4]]$

gap> GsHNPfalse44C2xC2: $=$ Filtered (GsHNPfalseC2xC2, $\mathrm{x}->\operatorname{List}(\operatorname{Orbits}(\mathrm{x},[1 \ldots 8])$,

$>$ Length $)=[4,4])$;

$[\operatorname{Group}([(1,2)(3,8)(4,6)(5,7),(1,8)(2,3)(4,5)(6,7)]), \operatorname{Group}([(1,3)(2,8)$

$(4,6)(5,7),(1,8)(2,3)(4,5)(6,7)]), \operatorname{Group}([(1,5)(2,6)(3,7)(4,8),(1,8)$

$(2,3)(4,5)(6,7)]), \operatorname{Group}([(),(1,8)(2,3)(4,5)(6,7),(1,7)(2,4)(3,5)$

$(6,8)]$ ] ]

gap> List (GsHNPtrueC2xC2, Elements);

$[[(),(1,2)(3,8)(4,7)(5,6),(1,4)(2,7)(3,6)(5,8),(1,7)(2,4)(3,5)(6,8)]$,

$[(),(1,2)(3,8)(4,7)(5,6),(1,5)(2,6)(3,7)(4,8),(1,6)(2,5)(3,4)(7,8)]$,

$[(),(1,3)(2,8)(4,6)(5,7),(1,4)(2,7)(3,6)(5,8),(1,6)(2,5)(3,4)(7,8)]$,

$[(),(1,3)(2,8)(4,6)(5,7),(1,5)(2,6)(3,7)(4,8),(1,7)(2,4)(3,5)(6,8)]]$ gap> List (GsHNPfalseC2xC2, Elements);

$[[(),(4,5)(6,7),(1,2)(3,8)(4,6)(5,7),(1,2)(3,8)(4,7)(5,6)]$,

$[(),(4,5)(6,7),(1,3)(2,8)(4,6)(5,7),(1,3)(2,8)(4,7)(5,6)]$,

$[(),(4,5)(6,7),(1,8)(2,3),(1,8)(2,3)(4,5)(6,7)]$,

$[(),(1,2)(3,8)(4,6)(5,7),(1,3)(2,8)(4,6)(5,7),(1,8)(2,3)]$,

$[(),(1,2)(3,8)(4,6)(5,7),(1,3)(2,8)(4,7)(5,6),(1,8)(2,3)(4,5)(6,7)]$,

$[(),(1,2)(3,8)(4,7)(5,6),(1,3)(2,8)(4,6)(5,7),(1,8)(2,3)(4,5)(6,7)]$,

$[(),(1,2)(3,8)(4,7)(5,6),(1,3)(2,8)(4,7)(5,6),(1,8)(2,3)]$,

$[(),(1,4)(2,7)(3,6)(5,8),(1,5)(2,6)(3,7)(4,8),(1,8)(2,3)(4,5)(6,7)]$,

$[(),(1,6)(2,5)(3,4)(7,8),(1,7)(2,4)(3,5)(6,8),(1,8)(2,3)(4,5)(6,7)]]$

gap> List (GsHNPfalse44C2xC2, Elements);

$[[(),(1,2)(3,8)(4,6)(5,7),(1,3)(2,8)(4,7)(5,6),(1,8)(2,3)(4,5)(6,7)]$,

$[(),(1,2)(3,8)(4,7)(5,6),(1,3)(2,8)(4,6)(5,7),(1,8)(2,3)(4,5)(6,7)]$,

$[(),(1,4)(2,7)(3,6)(5,8),(1,5)(2,6)(3,7)(4,8),(1,8)(2,3)(4,5)(6,7)]$,

$[(),(1,6)(2,5)(3,4)(7,8),(1,7)(2,4)(3,5)(6,8),(1,8)(2,3)(4,5)(6,7)]]$

gap> DG:=DerivedSubgroup $(G)$;

$\operatorname{Group}([(1,8)(2,3)(4,5)(6,7)])$

gap> List (GsHNPtrueC2xC2, $->$ Intersection $(\mathrm{x}, \mathrm{DG}))$;

[ $\operatorname{Group}(()), \operatorname{Group}(()), \operatorname{Group}(()), \operatorname{Group}(())]$

gap> List (GsHNPfalse44C2xC2, $\mathrm{x}->\operatorname{Intersection}(\mathrm{x}, \mathrm{DG}))$;

$[\operatorname{Group}([(1,8)(2,3)(4,5)(6,7)]), \operatorname{Group}([(1,8)(2,3)(4,5)(6,7)])$,

$\operatorname{Group}([(1,8)(2,3)(4,5)(6,7)]), \operatorname{Group}([(1,8)(2,3)(4,5)(6,7)])]$

$(2-2) G=8 T 11 \simeq\left(C_{4} \times C_{2}\right) \rtimes C_{2}$.

gap> Read("HNP.gap") ;

gap> G:=TransitiveGroup $(8,11) ; \# \mathrm{G}=8 \mathrm{~T} 11=(\mathrm{C} 4 \mathrm{xC} 2): \mathrm{C} 2$

$1 / 2\left[2\right.$-3] $\mathrm{E}(4)=\mathrm{Q} \_8: 2$

gap> GeneratorsOfGroup (G) ;

$[(1,5)(3,7),(1,3,5,7)(2,4,6,8),(1,4,5,8)(2,3,6,7)]$

gap> $\mathrm{H}:=\operatorname{Stabilizer}(\mathrm{G}, 1) ; \# \mathrm{H}=\mathrm{C} 2$

$\operatorname{Group}([(2,6)(4,8)])$

gap> FirstObstructionN $(G)$. ker; \# Obs1N=1

[ [ ], [ [ 2 ], [ ] ] ]

gap> SchurMultPcpGroup $(G) ; \# M(G)=C 2 \times C 2$ : Schur multiplicer of $G$

$[2,2]$

gap> cGs:=MinimalStemExtensions $(G) ; ; \# 3$ minimal stem extensions

gap> for $\mathrm{CG}$ in cGs do

$>\mathrm{bG}:=\mathrm{cG}$.MinimalStemExtension;

$>\mathrm{bH}:=\operatorname{PreImage}(\mathrm{cG}$. epi, $\mathrm{H})$;

$>\operatorname{Print}(\operatorname{KerResH} 3 \mathrm{Z}(\mathrm{bG}, \mathrm{bH}))$;

$>\operatorname{Print}(" \backslash \mathrm{n} ")$;

$>$ od;

[ [ 2$],[[2,2,2],[[0,0,1]]]]$

$[[],[[2,2],[]]]$ 
$[[2],[[2,2],[[0,1]]]]$

gap> for $c G$ in $c G s$ do

$>\mathrm{bG}:=\mathrm{cG}$.MinimalStemExtension;

$>\mathrm{bH}:=$ PreImage (cG.epi, $\mathrm{H})$;

$>$ Print (FirstObstructionN (bG,bH).ker [1]);

$>$ Print (FirstObstructionDnr (bG,bH).Dnr [1]);

$>$ Print("\n");

$>$ od;

[ 2 ] [ 2 ]

[ 2 ] [ ]

[ 2 ] [ 2 ]

gap> $c G:=c G s[2]$;

rec( MinimalStemExtension := <permutation group of size 32 with 5 generators>, epi $:=[(1,5,6,16)(2,9,10,22)(3,12,13,25)(4,14,15,26)(7,18,19,29)(8,20,21$, 30) $(11,23,24,31)(17,27,28,32),(1,3,6,13)(2,7,10,19)(4,12,15,25)(5,11$, $16,24)(8,18,21,29)(9,17,22,28)(14,23,26,31)(20,27,30,32)$, $(1,2)(3,7)(4,21)(5,22)(6,10)(8,15)(9,16)(11,28)(12,29)(13,19)(14,20)(17$, 24) $(18,25)(23,27)(26,30)(31,32)] \rightarrow$

$[(1,4,5,8)(2,3,6,7),(1,3,5,7)(2,4,6,8),(2,6)(4,8)])$

gap> bG:=cG.MinimalStemExtension; \# bG=G- is a minimal stem extension of $G$ <permutation group of size 32 with 5 generators>

gap> bH:=PreImage (cG.epi, H); \# bH=H-

Group $([(1,2)(3,7)(4,21)(5,22)(6,10)(8,15)(9,16)(11,28)(12,29)(13,19)(14,20)$

$(17,24)(18,25)(23,27)(26,30)(31,32),(1,26)(2,30)(3,31)(4,5)(6,14)(7,32)(8,9)$

$(10,20)(11,12)(13,23)(15,16)(17,18)(19,27)(21,22)(24,25)(28,29)])$

gap> FirstObstructionN (bG,bH).ker; \# Obs $1 \mathrm{~N}-=\mathrm{C} 2$

[ [ 2$],[[2,2],[[0,1]]]]$

gap> FirstObstructionDnr (bG,bH).Dnr; \# Obs1Dnr-=1

[ [ ], [ [ 2, 2 ], [ ] ] ]

gap> bGs:=AllSubgroups $(b G) ;$;

gap> Length(bGs);

58

gap> bGsHNPfalse:=Filtered(bGs, $\mathrm{x}->$ FirstObstructionDr (bG, $\mathrm{x}, \mathrm{bH}) \cdot \operatorname{Dr}[1]=[]$ ); ;

gap> Length (bGsHNPfalse);

55

gap> bGsHNPtrue:=Filtered(bGs, x->FirstObstructionDr (bG, x, bH).Dr[1] =[2] ); ;

gap> Length(bGsHNPtrue);

3

gap> Collected (List (bGsHNPfalse, $\mathrm{x}->$ StructureDescription (Image (cG.epi, $\mathrm{x}$ )))) ;

[ [ "1", 2 ], [ "C2", 17 ], [ "C2 x C2", 11 ], [ "C4", 12 ], [ "C4 x C2", 5 ], [ "D8", 7 ], [ "Q8", 1 ] ]

gap> Collected (List (bGsHNPtrue, $\mathrm{x}->$ StructureDescription(Image (cG.epi, $\mathrm{x})$ ))) ;

[ [ "(C4 x C2) : C2", 1 ], [ "C4 x C2", 2 ] ]

gap> GsHNPfalse: $=$ Set (bGsHNPfalse, $x->\operatorname{Image}(c G . e p i, x)) ;$;

gap> Length (GsHNPfalse);

20

gap> GsHNPtrue: $=$ Set (bGsHNPtrue, $\mathrm{x}->\operatorname{Image}(\mathrm{cG}$. epi, $\mathrm{x}))$; ;

gap> Length (GsHNPtrue);

3

gap> Intersection(GsHNPfalse, GsHNPtrue);

[ ]

gap> GsHNPtrueMin:=Filtered (GsHNPtrue, $\mathrm{x}->$ Length (Filtered (GsHNPtrue,

$>\mathrm{y}->\operatorname{IsSubgroup}(\mathrm{x}, \mathrm{y})))=1)$;

[ $\operatorname{Group}([(),(1,5)(2,6)(3,7)(4,8),(1,4,5,8)(2,3,6,7),(1,3,5,7)$

$(2,4,6,8)]), \operatorname{Group}([(),(1,5)(2,6)(3,7)(4,8),(1,3,5,7)(2,4,6,8),(1,8)$

$(2,3)(4,5)(6,7)])]$

gap> Length (GsHNPtrueMin);

2

gap> List (GsHNPtrueMin, IdSmallGroup);

$[$ [ 8,2$],[8,2]]$

gap>Collected(List (GsHNPfalse, $\mathrm{x}->$ Filtered (GsHNPtrueMin, $y->\operatorname{IsSubgroup}(\mathrm{x}, \mathrm{y})$ ))) )

$[[[], 20]]$

gap> Gs:=AllSubgroups (G); ; 


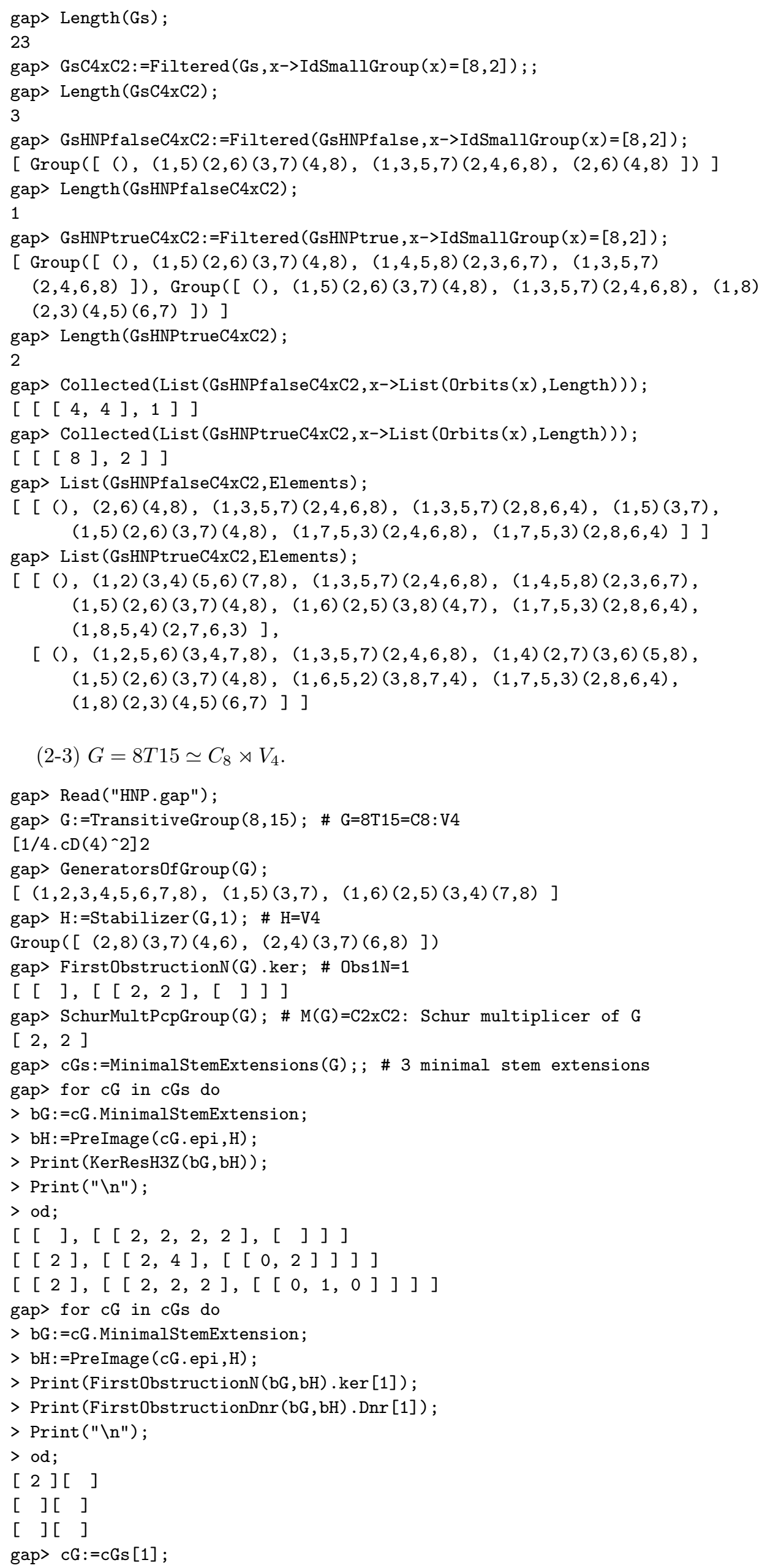


rec( MinimalStemExtension := <permutation group of size 64 with 3 generators , epi $:=[(3,4)(5,6)(7,9)(8,10)(11,13)(12,14)(15,18)(17,19)(20,23)(22,24)(25$, 27) $(28,30),(1,2)(3,7)(4,9)(5,11)(6,13)(8,12)(10,14)(15,20)(16,21)(17$, 25) $(18,23)(19,27)(22,28)(24,30)(26,29)(31,32)$, $(1,3,8,17,26,19,10,4)(2,5,12,22,29,24,14,6)(7,15,25,31,27,18,9,16)(11$, $20,28,32,30,23,13,21)] \rightarrow$

$[(2,8)(3,7)(4,6),(2,6)(4,8),(1,2,3,4,5,6,7,8)])$

gap> bG:=cG.MinimalStemExtension; \# bG=G- is a minimal stem extension of $G$ <permutation group of size 64 with 3 generators>

gap> bH:=PreImage (cG.epi,H); \# bH=H-

<permutation group of size 8 with 3 generators>

gap> FirstObstructionN (bG,bH).ker; \# Obs1N-=C2

[ [ 2$],[[2,2,2],[[0,0,1]]]]$

gap> FirstObstructionDnr(bG,bH).Dnr; \# Obs1Dnr-=1

[ [ ], [ [ 2, 2, 2 ], [ ] ] ]

gap> bGs:=AllSubgroups $(b G) ;$;

gap> Length(bGs);

225

gap> bGsHNPfalse:=Filtered(bGs, x->FirstObstructionDr (bG, x, bH).Dr [1] =[]); ;

gap> Length(bGsHNPfalse);

174

gap> bGsHNPtrue:=Filtered(bGs, x->FirstObstructionDr (bG, x, bH).Dr[1]=[2] ); ;

gap> Length(bGsHNPtrue);

51

gap> Collected (List (bGsHNPfalse, $x->$ StructureDescription (Image (cG.epi, x)))) ;

[ [ "1", 2$],[$ [ $22 ", 45],[$ "C2 x C2", 55 ], [ "C4", 12 ], [ "C4 x C2", 5 ], [ "C8", 6 ], [ "C8 : C2", 1 ], [ "D16", 10 ], [ "D8", 35 ], [ "Q8", 1 ],

[ "QD16", 2 ] ]

gap> Collected (List (bGsHNPtrue, $\mathrm{x}->$ StructureDescription (Image (cG.epi, $\mathrm{x})$ ))) ;

[ [ "(C4 x C2): $\mathrm{C} 2 ", 1],[$ "C2 x C2", 20$],[$ "C2 x C2 x C2", 18$]$,

[ "C2 x D8", 9 ], [ "C4 x C2", 2 ], [ "C8 : (C2 x C2)", 1 ] ]

gap> GsHNPfalse:=Set (bGsHNPfalse, $x->\operatorname{Image}(c G . e p i, x))$; ;

gap> Length(GsHNPfalse);

47

gap> GsHNPtrue:=Set (bGsHNPtrue, $\mathrm{x}->\operatorname{Image}(\mathrm{cG}$.epi, $\mathrm{x}))$; ;

gap> Length (GsHNPtrue);

11

gap> Intersection(GsHNPfalse, GsHNPtrue);

[ ]

gap> GsHNPtrueMin:=Filtered (GsHNPtrue, $\mathrm{x}->$ Length (Filtered (GsHNPtrue,

$>\mathrm{y}->\operatorname{IsSubgroup}(\mathrm{x}, \mathrm{y})))=1)$;

$[\operatorname{Group}([(1,5)(3,7),(1,5)(2,4)(6,8)]), \operatorname{Group}([(2,6)(4,8),(2,6)$

$(4,8),(1,3)(4,8)(5,7)]), \operatorname{Group}([(2,6)(4,8),(2,6)(4,8),(1,7)(2,6)$

$(3,5)]), \operatorname{Group}([(1,5)(3,7),(2,8)(3,7)(4,6)]), \operatorname{Group}([(1,5)(2,6)(3,7)$

$(4,8),(1,2)(3,8)(4,7)(5,6),(1,3,5,7)(2,8,6,4),(1,7,5,3)(2,4,6,8)])$,

Group $([(1,5)(2,6)(3,7)(4,8),(1,8)(2,7)(3,6)(4,5),(1,7,5,3)$

$(2,4,6,8),(1,3,5,7)(2,8,6,4)])]$

gap> Length(GsHNPtrueMin);

6

gap> List (GsHNPtrueMin, IdSmallGroup);

$[[4,2],[4,2],[4,2],[4,2],[8,2],[8,2]]$

gap $>$ Collected (List (GsHNPfalse, $x->$ Filtered (GsHNPtrueMin, $y->\operatorname{IsSubgroup}(x, y))$ )) ;

[ [ [ ], 47 ] ]

gap> Gs:=AllSubgroups $(G) ;$;

gap> Length(Gs);

58

gap> GsC2xC2:=Filtered (Gs, $x->\operatorname{IdSmallGroup~}(\mathrm{x})=[4,2]) ;$;

gap> Length (GsC2xC2);

15

gap> GsC4xC2: $=$ Filtered $(\mathrm{Gs}, \mathrm{x}->\operatorname{IdSmallGroup}(\mathrm{x})=[8,2])$; ;

gap> Length (GsC4xC2);

3

gap> GsHNPfalseC2xC2:=Filtered(GsHNPfalse, $\mathrm{x}->\operatorname{IdSmallGroup}(\mathrm{x})=[4,2])$; 
[ $\operatorname{Group}([(2,6)(4,8),(2,4)(3,7)(6,8)]), \operatorname{Group}([(2,4)(3,7)(6,8),(1,5)(2,8)$ $(4,6)]), \operatorname{Group}([(1,5)(2,6)(3,7)(4,8),(1,5)(3,7),(2,6)(4,8)])$, $\operatorname{Group}([(2,6)(4,8),(2,6)(4,8),(1,5)(2,4)(6,8)]), \operatorname{Group}([(1,5)(2,6)(3,7)$ $(4,8),(1,5)(2,6)(3,7)(4,8),(2,8)(3,7)(4,6)]), \operatorname{Group}([(1,5)(2,6)(3,7)$ $(4,8),(1,2)(3,8)(4,7)(5,6)]), \operatorname{Group}([(1,5)(3,7),(1,5)(3,7),(1,3)(4,8)$ $(5,7)]), \operatorname{Group}([(1,5)(2,6)(3,7)(4,8),(1,5)(2,6)(3,7)(4,8),(1,3)(4,8)$ $(5,7)]), \operatorname{Group}([(1,5)(3,7),(1,5)(3,7),(1,7)(2,6)(3,5)]), \operatorname{Group}([(1,5)$ $(2,6)(3,7)(4,8),(1,3)(2,6)(5,7),(1,7)(3,5)(4,8)]), \operatorname{Group}([(1,5)(2,6)$ $(3,7)(4,8),(1,8)(2,7)(3,6)(4,5),(1,5)(2,6)(3,7)(4,8)])]$ gap> Length (GsHNPfalseC2xC2);

11 gap> GsHNPtrueC2xC2: $=$ Filtered (GsHNPtrue, $x->\operatorname{IdSmallGroup}(\mathrm{x})=[4,2])$;

$[\operatorname{Group}([(1,5)(3,7),(1,5)(2,4)(6,8)]), \operatorname{Group}([(2,6)(4,8),(2,6)$

$(4,8),(1,3)(4,8)(5,7)]), \operatorname{Group}([(2,6)(4,8),(2,6)(4,8),(1,7)(2,6)$

$(3,5)]), \operatorname{Group}([(1,5)(3,7),(2,8)(3,7)(4,6)])]$ gap> Length(GsHNPtrueC2xC2);

4

gap> Collected(List(GsHNPfalseC2xC2, x->List(Orbits (x), Length)));

$[[[2,2,2,2], 1],[[2,4], 1],[[2,4,2], 2],[[4,2], 3]$, $[[4,2,2], 2],[[4,4], 2]]$

gap> Collected(List(GsHNPtrueC2xC2, $x->\operatorname{List}(\operatorname{Orbits}(\mathrm{x})$, Length)));

$[[[2,2,2,2], 4]]$

gap> GsHNPfalse2222C2xC2:=Filtered(GsHNPfalseC2xC2,

$>\mathrm{x}->\operatorname{List}(\operatorname{Orbits}(\mathrm{x},[1 \ldots 8])$, Length) $=[2,2,2,2])$;

$[\operatorname{Group}([(1,5)(2,6)(3,7)(4,8),(1,5)(3,7),(2,6)(4,8)])]$

gap> List (GsHNPtrueC2xC2, Elements);

$[[(),(2,4)(3,7)(6,8),(1,5)(3,7),(1,5)(2,4)(6,8)]$,

$[(),(2,6)(4,8),(1,3)(4,8)(5,7),(1,3)(2,6)(5,7)]$,

$[(),(2,6)(4,8),(1,7)(3,5)(4,8),(1,7)(2,6)(3,5)]$,

$[(),(2,8)(3,7)(4,6),(1,5)(3,7),(1,5)(2,8)(4,6)]]$

gap> List (GsHNPfalseC2xC2, Elements);

$[[(),(2,4)(3,7)(6,8),(2,6)(4,8),(2,8)(3,7)(4,6)]$,

$[(),(2,4)(3,7)(6,8),(1,5)(2,6)(3,7)(4,8),(1,5)(2,8)(4,6)]$,

$[(),(2,6)(4,8),(1,5)(3,7),(1,5)(2,6)(3,7)(4,8)]$,

$[(),(2,6)(4,8),(1,5)(2,4)(6,8),(1,5)(2,8)(4,6)]$,

$[(),(2,8)(3,7)(4,6),(1,5)(2,4)(6,8),(1,5)(2,6)(3,7)(4,8)]$,

$[(),(1,2)(3,8)(4,7)(5,6),(1,5)(2,6)(3,7)(4,8),(1,6)(2,5)(3,4)(7,8)]$,

$[(),(1,3)(4,8)(5,7),(1,5)(3,7),(1,7)(3,5)(4,8)]$,

$[(),(1,3)(4,8)(5,7),(1,5)(2,6)(3,7)(4,8),(1,7)(2,6)(3,5)]$,

$[(),(1,3)(2,6)(5,7),(1,5)(3,7),(1,7)(2,6)(3,5)]$,

$[(),(1,3)(2,6)(5,7),(1,5)(2,6)(3,7)(4,8),(1,7)(3,5)(4,8)]$,

$[(),(1,4)(2,3)(5,8)(6,7),(1,5)(2,6)(3,7)(4,8),(1,8)(2,7)(3,6)(4,5)]]$

gap> List (GsHNPfalse2222C2xC2,Elements);

$[[(),(2,6)(4,8),(1,5)(3,7),(1,5)(2,6)(3,7)(4,8)]]$

gap> DG:=DerivedSubgroup $(G)$;

$\operatorname{Group}([(1,5)(2,6)(3,7)(4,8),(1,3,5,7)(2,4,6,8)])$

gap> List (GsHNPtrueC2xC2, $\mathrm{x}->\operatorname{Intersection}(\mathrm{x}, \mathrm{DG}))$;

[ $\operatorname{Group}(()), \operatorname{Group}(()), \operatorname{Group}(()), \operatorname{Group}(())]$

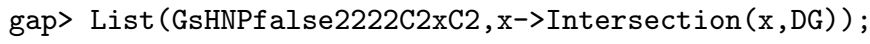

[ $\operatorname{Group}([(1,5)(2,6)(3,7)(4,8)])]$

gap> A8:=AlternatingGroup (8);

$\operatorname{Alt}\left(\left[\begin{array}{llll}1 & \ldots & 8\end{array}\right]\right)$

gap> List (GsHNPtrueC2xC2, $\mathrm{x}->$ IsSubgroup $(\mathrm{A} 8, \mathrm{x})$ ) ;

[ false, false, false, false ]

gap> List (GsHNPfalse2222C2xC2, $\mathrm{x}->\operatorname{IsSubgroup}(\mathrm{A} 8, \mathrm{x})$ ) ;

[ true ]

gap> GsHNPfalseC4xC2:=Filtered (GsHNPfalse, $x->\operatorname{IdSmallGroup}(\mathrm{x})=[8,2]$ ) ;

$[\operatorname{Group}([(1,5)(2,6)(3,7)(4,8),(2,6)(4,8),(1,7,5,3)(2,8,6,4)])]$

gap> Length (GsHNPfalseC4xC2);

1

gap> GsHNPtrueC4xC2: $=$ Filtered (GsHNPtrue, $\mathrm{x}->\operatorname{IdSmallGroup}(\mathrm{x})=[8,2]$ ) ;

$[\operatorname{Group}([(1,5)(2,6)(3,7)(4,8),(1,2)(3,8)(4,7)(5,6),(1,3,5,7)$

$(2,8,6,4),(1,7,5,3)(2,4,6,8)]), \operatorname{Group}([(1,5)(2,6)(3,7)(4,8),(1,8)(2,7)$ 


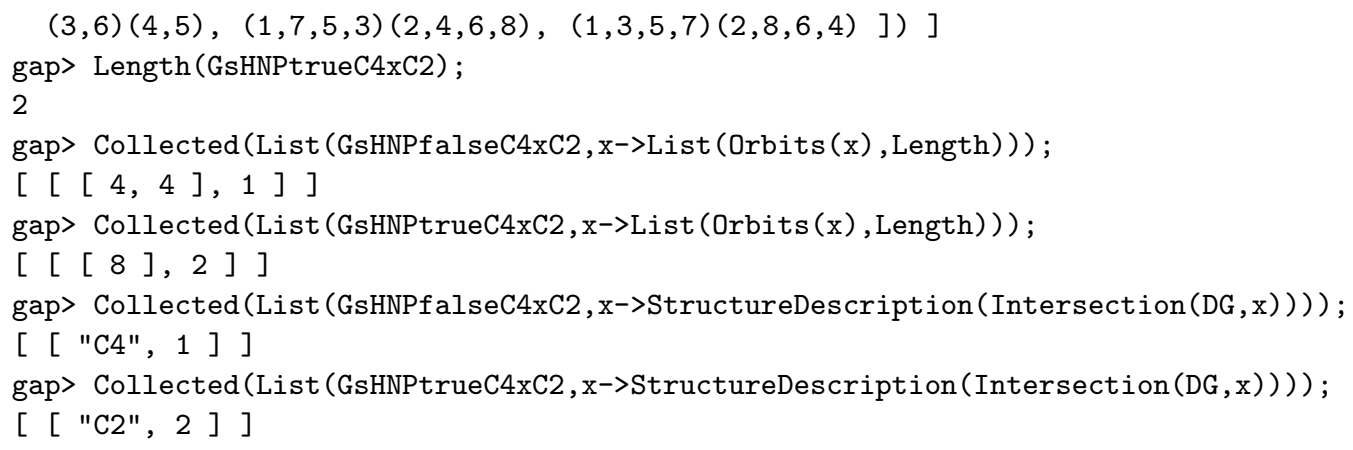


105

gap $>$ bGsHNPfalse:=Filtered(bGs, $\mathrm{x}->$ FirstObstructionDr $(\mathrm{bG}, \mathrm{x}, \mathrm{bH}) \cdot \operatorname{Dr}[1]=[])$; ; gap> Length(bGsHNPfalse);

86

gap> bGsHNPtrue:=Filtered(bGs, x->FirstObstructionDr (bG, x, bH).Dr[1]=[2] ); ; gap> Length(bGsHNPtrue);

19

gap> Collected (List (bGsHNPfalse, $\mathrm{x}->$ StructureDescription (Image (cG.epi, $\mathrm{x}$ )))) ;

[ [ "(C4 x C2) : C2", 1 ], [ "1", 2 ], [ "C2", 25 ], [ "C2 x C2", 21 ],

[ "C2 x C2 x C2", 1 ], [ "C4", 22 ], [ "C4 x C2", 2 ], [ "D8", 12 ] ]

gap> Collected (List (bGsHNPtrue, $\mathrm{x}->$ StructureDescription (Image (cG.epi,x)))) ;

[ [ "(C2 x C2 x C2 $): \mathrm{C} 4 ", 1],[$ " $(\mathrm{C} 4 \times \mathrm{C} 2): \mathrm{C} 2 ", 1],[$ "C2 x C2", 4 ],

[ "C2 x C2 x C2", 1 ], [ "C2 x D8", 1 ], [ "C4 x C2", 11 ] ]

gap> GsHNPfalse:=Set (bGsHNPfalse, $x->\operatorname{Image}(c G . e p i, x))$; ;

gap> Length (GsHNPfalse);

39

gap> GsHNPtrue:=Set (bGsHNPtrue, $\mathrm{x}->$ Image $(\mathrm{cG}$. epi, $\mathrm{x})$ ) ; ;

gap> Length (GsHNPtrue);

11

gap> Intersection(GsHNPfalse, GsHNPtrue);

[ ]

gap> GsHNPtrueMin:=Filtered(GsHNPtrue, $\mathrm{x}->$ Length(Filtered (GsHNPtrue,

$>\mathrm{y}->\operatorname{IsSubgroup}(\mathrm{x}, \mathrm{y})))=1)$;

$[\operatorname{Group}([(1,3)(4,7,6,5),(1,3)(2,8)(4,6)(5,7),(4,6)(5,7)])$,

$\operatorname{Group}([(1,8,3,2)(5,7),(1,3)(2,8)(4,6)(5,7),(1,3)(2,8)])$,

$\operatorname{Group}([(1,7,3,5)(2,4,8,6),(1,2)(3,8)(4,7)(5,6),(),(1,3)(2,8)(4,6)$

$(5,7)]), \operatorname{Group}([(1,4)(2,7)(3,6)(5,8),(1,2)(3,8)(4,7)(5,6),()])$,

Group $([(1,6)(2,5)(3,4)(7,8),(1,2)(3,8)(4,7)(5,6),()]), \operatorname{Group}([(1,5)$

$(2,6)(3,7)(4,8),(1,8)(2,3)(4,5)(6,7),()]), \operatorname{Group}([(1,7)(2,4)(3,5)$

$(6,8),(1,8)(2,3)(4,5)(6,7),()])]$

gap> Length(GsHNPtrueMin);

7

gap> List (GsHNPtrueMin, IdSmallGroup);

$[[8,2],[8,2],[8,2],[4,2],[4,2],[4,2],[4,2]]$

gap> Gs:=AllSubgroups $(G) ;$;

gap> Length(Gs);

50

gap $>$ GsC2xC2: $=$ Filtered (Gs, $x->\operatorname{IdSmallGroup}(\mathrm{x})=[4,2])$;

$[\operatorname{Group}([(1,2)(3,8)(4,7)(5,6),(1,8)(2,3)(4,5)(6,7)]), \operatorname{Group}([(1,4)(2,7)$

$(3,6)(5,8),(1,2)(3,8)(4,7)(5,6)]), \operatorname{Group}([(1,6)(2,5)(3,4)(7,8),(1,2)$

$(3,8)(4,7)(5,6)]), \operatorname{Group}([(1,5)(2,6)(3,7)(4,8),(1,8)(2,3)(4,5)(6,7)])$,

Group $([(1,7)(2,4)(3,5)(6,8),(1,8)(2,3)(4,5)(6,7)]), \operatorname{Group}([(1,4)(2,7)$

$(3,6)(5,8),(1,3)(2,8)(4,6)(5,7)]), \operatorname{Group}([(1,5)(2,6)(3,7)(4,8),(1,3)$

$(2,8)(4,6)(5,7)]), \operatorname{Group}([(4,6)(5,7),(1,2)(3,8)(4,7)(5,6)])$,

$\operatorname{Group}([(4,6)(5,7),(1,8)(2,3)(4,5)(6,7)]), \operatorname{Group}([(1,3)(2,8),(1,8)(2,3)$

$(4,5)(6,7)]), \operatorname{Group}([(1,3)(2,8),(1,2)(3,8)(4,7)(5,6)]), \operatorname{Group}([(4,6)$

$(5,7),(1,3)(2,8)(4,6)(5,7)]), \operatorname{Group}([(1,8)(2,3)(4,7)(5,6),(1,3)(2,8)$

$(4,6)(5,7)]$ ] ]

gap> Length (GsC2xC2);

13

gap> GsC4xC2:=Filtered (Gs, $\mathrm{x}->\operatorname{IdSmallGroup}(\mathrm{x})=[8,2])$;

$[\operatorname{Group}([(1,2)(3,8)(4,7)(5,6),(1,8)(2,3)(4,5)(6,7),(1,5,3,7)(2,6,8,4)])$, Group $([(2,8)(4,7,6,5),(4,6)(5,7),(1,3)(2,8)(4,6)(5,7)])$,

Group $([(1,8,3,2)(4,6),(1,3)(2,8),(1,3)(2,8)(4,6)(5,7)])$,

$\operatorname{Group}([(1,4,2,5)(3,6,8,7),(1,2)(3,8)(4,5)(6,7),(1,3)(2,8)(4,6)(5,7)])$,

$\operatorname{Group}([(1,5,8,6)(2,4,3,7),(1,8)(2,3)(4,7)(5,6),(1,3)(2,8)(4,6)(5,7)])]$ gap> Length (GsC4xC2);

5

gap> GsHNPfalseC2xC2:=Filtered(GsHNPfalse, $x->\operatorname{IdSmallGroup}(\mathrm{x})=[4,2])$;

$[\operatorname{Group}([(1,2)(3,8)(4,5)(6,7),(4,6)(5,7),(4,6)(5,7)]), \operatorname{Group}([(1,3)(2,8)$

$(4,6)(5,7),(1,3)(2,8),(4,6)(5,7)]), \operatorname{Group}([(1,8)(2,3)(4,7)(5,6),(4,6)$

$(5,7),(4,6)(5,7)]), \operatorname{Group}([(1,2)(3,8)(4,5)(6,7),(1,3)(2,8),(1,3)$

$(2,8)]), \operatorname{Group}([(1,2)(3,8)(4,5)(6,7),(1,3)(2,8)(4,6)(5,7),(1,3)(2,8)$ 
$(4,6)(5,7)]), \operatorname{Group}([(1,8)(2,3)(4,7)(5,6),(1,3)(2,8),(1,3)(2,8)])$, $\operatorname{Group}([(1,2)(3,8)(4,7)(5,6),(1,3)(2,8)(4,6)(5,7),(1,3)(2,8)(4,6)$ $(5,7)]), \operatorname{Group}([(1,4)(2,7)(3,6)(5,8),(1,3)(2,8)(4,6)(5,7)])$, $\operatorname{Group}([(1,7)(2,4)(3,5)(6,8),(1,3)(2,8)(4,6)(5,7)])]$ gap> Length(GsHNPfalseC2xC2);

9

gap> GsHNPtrueC2xC2:=Filtered (GsHNPtrue, $\mathrm{x}->\operatorname{IdSmallGroup}(\mathrm{x})=[4,2]$ ) ;

$[\operatorname{Group}([(1,4)(2,7)(3,6)(5,8),(1,2)(3,8)(4,7)(5,6),()]), \operatorname{Group}([(1,6)$

$(2,5)(3,4)(7,8),(1,2)(3,8)(4,7)(5,6),()]), \operatorname{Group}([(1,5)(2,6)(3,7)$

$(4,8),(1,8)(2,3)(4,5)(6,7),()]), \operatorname{Group}([(1,7)(2,4)(3,5)(6,8),(1,8)(2,3)$

$(4,5)(6,7),()])]$

gap> Length (GsHNPtrueC2xC2);

4

gap> Collected (List (GsHNPfalseC2xC2, $\mathrm{x}->\operatorname{List}(\operatorname{Orbits}(\mathrm{x})$, Length))) ;

$[[[2,2,2,2], 1],[[2,2,4], 2],[[4,2,2], 2]$,

$[[4,4], 4]]$

gap> Collected(List (GsHNPtrueC2xC2, x->List (Orbits (x), Length))) ;

[ [ [ 4, 4 ], 4 ] ]

gap> GsHNPfalse44C2xC2:=Filtered(GsHNPfalseC2xC2,

$>\mathrm{x}->\operatorname{List}(\operatorname{Orbits}(\mathrm{x},[1 \ldots 8])$, Length) $=[4,4])$;

$[\operatorname{Group}([(1,2)(3,8)(4,5)(6,7),(1,3)(2,8)(4,6)(5,7),(1,3)(2,8)(4,6)$

$(5,7)]), \operatorname{Group}([(1,2)(3,8)(4,7)(5,6),(1,3)(2,8)(4,6)(5,7),(1,3)(2,8)$

$(4,6)(5,7)]), \operatorname{Group}([(1,4)(2,7)(3,6)(5,8),(1,3)(2,8)(4,6)(5,7)])$,

$\operatorname{Group}([(1,7)(2,4)(3,5)(6,8),(1,3)(2,8)(4,6)(5,7)])]$

gap> List (GsHNPtrueC2xC2, Elements);

$[[(),(1,2)(3,8)(4,7)(5,6),(1,4)(2,7)(3,6)(5,8),(1,7)(2,4)(3,5)(6,8)]$,

$[(),(1,2)(3,8)(4,7)(5,6),(1,5)(2,6)(3,7)(4,8),(1,6)(2,5)(3,4)(7,8)]$,

$[(),(1,4)(2,7)(3,6)(5,8),(1,5)(2,6)(3,7)(4,8),(1,8)(2,3)(4,5)(6,7)]$,

$[(),(1,6)(2,5)(3,4)(7,8),(1,7)(2,4)(3,5)(6,8),(1,8)(2,3)(4,5)(6,7)]]$

gap> List (GsHNPfalseC2xC2,Elements);

[ [ ()$,(4,6)(5,7),(1,2)(3,8)(4,5)(6,7),(1,2)(3,8)(4,7)(5,6)]$,

$[(),(4,6)(5,7),(1,3)(2,8),(1,3)(2,8)(4,6)(5,7)]$,

$[(),(4,6)(5,7),(1,8)(2,3)(4,5)(6,7),(1,8)(2,3)(4,7)(5,6)]$,

$[(),(1,2)(3,8)(4,5)(6,7),(1,3)(2,8),(1,8)(2,3)(4,5)(6,7)]$,

$[(),(1,2)(3,8)(4,5)(6,7),(1,3)(2,8)(4,6)(5,7),(1,8)(2,3)(4,7)(5,6)]$,

$[(),(1,2)(3,8)(4,7)(5,6),(1,3)(2,8),(1,8)(2,3)(4,7)(5,6)]$,

$[(),(1,2)(3,8)(4,7)(5,6),(1,3)(2,8)(4,6)(5,7),(1,8)(2,3)(4,5)(6,7)]$,

$[(),(1,3)(2,8)(4,6)(5,7),(1,4)(2,7)(3,6)(5,8),(1,6)(2,5)(3,4)(7,8)]$,

$[(),(1,3)(2,8)(4,6)(5,7),(1,5)(2,6)(3,7)(4,8),(1,7)(2,4)(3,5)(6,8)]]$

gap> List (GsHNPfalse44C2xC2, Elements);

$[[(),(1,2)(3,8)(4,5)(6,7),(1,3)(2,8)(4,6)(5,7),(1,8)(2,3)(4,7)(5,6)]$,

$[(),(1,2)(3,8)(4,7)(5,6),(1,3)(2,8)(4,6)(5,7),(1,8)(2,3)(4,5)(6,7)]$,

$[(),(1,3)(2,8)(4,6)(5,7),(1,4)(2,7)(3,6)(5,8),(1,6)(2,5)(3,4)(7,8)]$,

$[(),(1,3)(2,8)(4,6)(5,7),(1,5)(2,6)(3,7)(4,8),(1,7)(2,4)(3,5)(6,8)]]$

gap> ZG:=Centre $(G)$;

$\operatorname{Group}([(1,3)(2,8)(4,6)(5,7)])$

gap> List (GsHNPtrueC2xC2, $\mathrm{x}->$ Intersection $(\mathrm{x}, \mathrm{ZG}))$;

[ $\operatorname{Group}(()), \operatorname{Group}(()), \operatorname{Group}(()), \operatorname{Group}(())]$

gap> List (GsHNPfalse44C2xC2, $\mathrm{x}->$ Intersection $(\mathrm{x}, \mathrm{ZG})$ ) ;

$[\operatorname{Group}([(1,3)(2,8)(4,6)(5,7)]), \operatorname{Group}([(1,3)(2,8)(4,6)(5,7)])$,

$\operatorname{Group}([(1,3)(2,8)(4,6)(5,7)]), \operatorname{Group}([(1,3)(2,8)(4,6)(5,7)])]$

gap> UcsG:=UpperCentralSeries $(G)$;

$[\operatorname{Group}([(1,3)(2,8)(4,6)(5,7),(4,6)(5,7),(1,8)(2,3)(4,5)(6,7),(2,8)$

$(4,7,6,5),(1,5)(2,6)(3,7)(4,8)]), \operatorname{Group}([(1,3)(2,8)(4,6)(5,7),(4,6)$

$(5,7),(1,8)(2,3)(4,5)(6,7)]), \operatorname{Group}([(1,3)(2,8)(4,6)(5,7)]), \operatorname{Group}(())]$

gap> Collected (List (GsHNPfalseC2xC2, $\mathrm{x}->\operatorname{List}(\operatorname{UcsG}, \mathrm{y}->\operatorname{Order}(\operatorname{Intersection}(\mathrm{y}, \mathrm{x})))$ )) ;

$[[[4,2,2,1], 2],[[4,4,1,1], 4],[[4,4,2,1], 3]]$

gap> Collected (List (GsHNPtrueC2xC2, $\mathrm{x}->\operatorname{List}(\operatorname{UcsG}, \mathrm{y}->\operatorname{Order}(\operatorname{Intersection}(\mathrm{y}, \mathrm{x})))$ )) ;

$[[[4,2,1,1], 4]]$

gap> GsHNPfalseC4xC2: $=$ Filtered (GsHNPfalse, $x->\operatorname{IdSmallGroup}(\mathrm{x})=[8,2])$;

$[\operatorname{Group}([(1,4,2,5)(3,6,8,7),(1,2)(3,8)(4,5)(6,7),(),(1,3)(2,8)(4,6)$

$(5,7)]), \operatorname{Group}([(1,7,8,4)(2,6,3,5),(1,8)(2,3)(4,7)(5,6),(),(1,3)(2,8)$

$(4,6)(5,7)]$ ] ] 


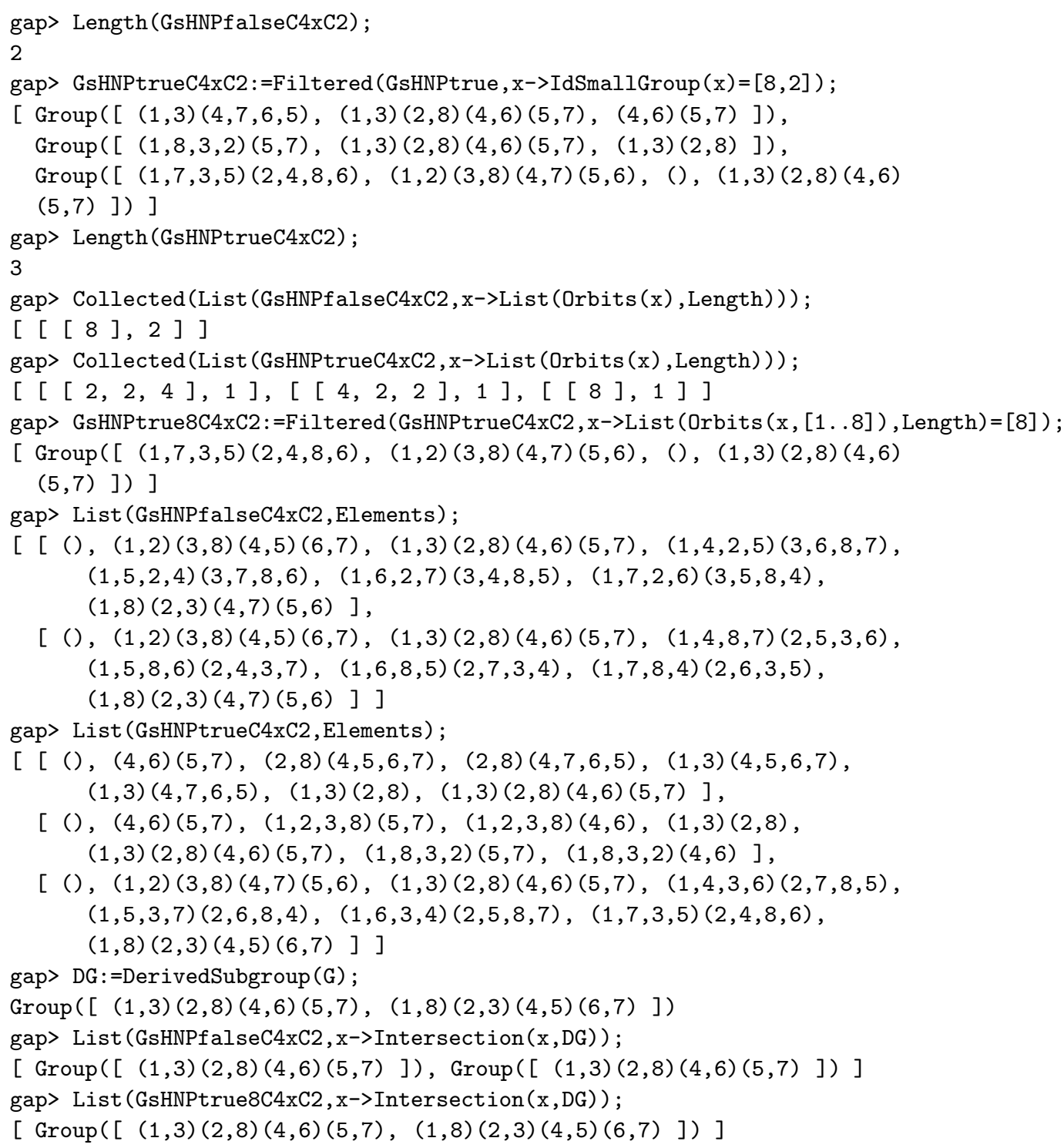


$[[2,2,2,2],[[0,1,0,0],[0,0,1,0],[0,0,0,1]]]]$

[ [ 2$],[[2,2,2,2,4],[[1,1,0,1,0]]]]$

[ [ 2$],[[2,2,2,2,2],[[1,1,0,1,0]]]]$

[ [ 2$],[[2,2,2,2,2,2],[[0,0,0,1,0,0]]]]$

[ [ 2,2$],[[2,2,2,2,2],[[0,1,0,0,0],[0,0,1,0,0]]]]$

[ [ 2$],[[2,2,2,2,2,2],[[0,0,1,0,0,1]]]]$

$[[2],[[2,2,2,2,4],[[0,1,0,1,2]]]]$

[ [ 2$],[[2,2,2,2,2,2],[[0,0,1,1,0,1]]]]$

[ [ 2,2$],[[2,2,2,2,4],[[0,0,1,1,0],[0,0,0,0,2]]]]$

[ [ 2$],[[2,2,2,2],[[0,0,1,1]]]]$

$[[2],[[2,2,2,2],[[1,0,1,1]]]]$

[ [ ] $][[2,2,2,2,2],[]]]$

[ [ 2$]],[[2,2,2,2,2],[[0,0,0,0,1]]]]$

$[[2],[[2,2,2,2],[[0,1,0,0]]]]$

[ [ 2$],[[2,2,2,2],[[0,0,0,1]]]]$

$[[2,2],[[2,2,2,2,2],[[0,1,0,0,0],[0,0,0,1,0]]]]$

$[[2,2,2]$,

$[[2,2,4,4],[[1,0,0,0],[0,0,2,0],[0,0,0,2]]]]$

[ [ 2$],[[2,2,2,2,4],[[0,0,1,1,0]]]]$

$[[2],[[2,2,2,2],[[1,0,1,0]]]]$

[ [ 2$],[[2,2,2,2,2],[[0,1,0,0,0]]]]$

$[[2,2],[[2,2,2,2],[[1,0,0,0],[0,0,0,1]]]]$

[ [ 2$],[[2,2,2,2],[[1,0,1,0]]]]$

$[[2],[[2,2,2,2],[[1,0,0,1]]]]$

$[[2],[[2,2,2,2,2,2],[[0,0,0,1,0,1]]]]$

$[[2,2],[[2,2,2,2,2],[[0,0,1,0,1],[0,0,0,1,1]]]]$

$[[2],[[2,2,2,2,2],[[0,0,1,0,0]]]]$

[ [ 2$],[[2,2,2,2],[[0,1,1,0]]]]$

gap> for $C G$ in $C G s$ do

$>\mathrm{bG}:=\mathrm{cG}$.MinimalStemExtension;

$>$ bH: $=$ PreImage (cG.epi, H);

$>$ Print (FirstObstructionN (bG, bH) . ker [1]);

$>$ Print (FirstObstructionDnr (bG, bH).Dnr [1]);

$>$ Print ("\n");

$>$ od;

[ 2 ] [ 2 ]

[ ] [ ]

[ ] [ ]

[ 2 ] [ 2 ]

[ 2 ][ 2 ]

[ ] [ ]

[ ] [ ]

[ 2 ] [ 2 ]

[ 2 ] [ 2 ]

[ ] [ ]

[ ][ ]

[ 2 ][ 2 ]

[ 2 ][ 2 ]

[ ] [ ]

[ ] [ ]

[ 2 ] [ ]

[ 2 ][ 2 ]

[ ] [ ]

[ ] [ ]

[ 2 ] [ 2 ]

[ 2 ][ 2 ]

[ ] [ ]

[ ] [ ]

[ 2 ][ 2 ]

[ 2 ][ 2 ]

[ ] [ ]

[ ] [ ]

[ 2 ][ 2 ] 
[ 2 ] [ 2 ]

$[\mathrm{b}]$

$[$ ] $]$

gap> $c G:=c G s[16] ;$;

gap> bG:=cG.MinimalStemExtension; \# $b G=G-$ is a minimal stem extension of $\mathrm{G}$

<permutation group of size 64 with 6 generators>

gap> bH:=PreImage (cG.epi,H); \# bH=H-

<permutation group of size 8 with 3 generators>

gap> FirstObstructionN (bG,bH).ker; \# Obs $1 \mathrm{~N}-=\mathrm{C} 2$

[ [ 2$],[[2,2,2],[[0,0,1]]]]$

gap> FirstObstructionDnr(bG,bH).Dnr; \# Obs $1 \mathrm{Dnr}-=1$

[ [ ], [ [ 2, 2, 2], [ ] ] ]

gap> bGs:=AllSubgroups (bG); ;

gap> Length(bGs);

321

gap> bGsHNPfalse: $=$ Filtered (bGs, $\mathrm{x}->$ FirstObstructionDr $(\mathrm{bG}, \mathrm{x}, \mathrm{bH}) \cdot \operatorname{Dr}[1]=[]) ;$;

gap> Length (bGsHNPfalse);

292

gap> bGsHNPtrue: $=$ Filtered (bGs, $\mathrm{x}->$ FirstObstructionDr (bG, $\mathrm{x}, \mathrm{bH})$. $\operatorname{Dr}[1]=[2]$ ) ; ;

gap> Length(bGsHNPtrue);

29

gap> Collected (List (bGsHNPfalse, $\mathrm{x}->$ StructureDescription (Image (cG.epi, $\mathrm{x}$ ))));

[ [ "1", 2 ], [ "C2", 49 ], [ "C2 x C2", 101 ], [ "C2 x C2 x C2", 28 ],

[ "C2 x D8", 19 ], [ "C4", 18 ], [ "C4 x C2", 15 ], [ "D8", 58 ],

[ "Q8", 2 ] ]

gap> Collected (List (bGsHNPtrue, $\mathrm{x}->$ StructureDescription(Image (cG. epi, $\mathrm{x}$ ))));

[ [ "(C2 x C2 x C2) : (C2 x C2)", 1$],[$ "(C4 x C2) : C2", 6$]$,

[ "C2 $\times$ C2", 8 ], [ "C2 $\times \mathrm{C} 2 \times \mathrm{C} 2 ", 2]$, [ "C2 $\times$ D8", 6 ],

[ "C4 x C2", 6 ] ]

gap> GsHNPfalse: $=\operatorname{Set}($ bGsHNPfalse, $\mathrm{x}->\operatorname{Image}(\mathrm{cG} . \mathrm{epi}, \mathrm{x})$ ); ;

gap> Length (GsHNPfalse);

81

gap> GsHNPtrue:=Set (bGsHNPtrue, $\mathrm{x}->\operatorname{Image}(\mathrm{cG}$. epi, $\mathrm{x})$ ) ; ;

gap> Length(GsHNPtrue);

29

gap> Intersection(GsHNPfalse, GsHNPtrue);

[ ]

gap> GsHNPtrueMin:=Filtered (GsHNPtrue, $\mathrm{x}$->Length(Filtered (GsHNPtrue,

$>\mathrm{y}->\operatorname{IsSubgroup}(\mathrm{x}, \mathrm{y})))=1)$;

[ Group $([(),(1,8)(2,3)(4,5)(6,7),(1,2)(3,8)(4,6)(5,7),(1,4,8,5)$

$(2,6,3,7)])$, Group $([(1,4)(2,6)(3,7)(5,8),(1,6)(2,4)(3,5)(7,8),()])$, Group $([(1,5)(2,7)(3,6)(4,8),(1,7)(2,5)(3,4)(6,8),()])$,

Group ( $[(),(1,8)(2,3)(4,5)(6,7),(1,6,8,7)(2,5,3,4),(1,2)(3,8)(4,7)$

$(5,6)])$, Group $([(1,2)(3,8)(4,7)(5,6),(1,7)(2,4)(3,5)(6,8),()])$,

Group $([(1,2)(3,8)(4,7)(5,6),(1,6)(2,5)(3,4)(7,8),()])$,

Group $([(),(1,8)(2,3)(4,5)(6,7),(1,4)(2,6)(3,7)(5,8),(1,2,8,3)$

$(4,6,5,7)])$, Group $([(),(1,8)(2,3)(4,5)(6,7),(1,4,8,5)(2,6,3,7),(1,2,8$,

3) $(4,6,5,7)])$, Group $([(),(1,8)(2,3)(4,5)(6,7),(1,2,8,3)$

$(4,7,5,6),(1,4)(2,7)(3,6)(5,8)]), \operatorname{Group}([(),(1,8)(2,3)(4,5)$

$(6,7),(1,2,8,3)(4,7,5,6),(1,6)(2,4)(3,5)(7,8)])$, Group $([(1,3)(2,8)(4,6)$

$(5,7),(1,6)(2,5)(3,4)(7,8),()]), \operatorname{Group}([(1,3)(2,8)(4,6)(5,7),(1,7)$

$(2,4)(3,5)(6,8),()]), \operatorname{Group}([(1,4)(2,6)(3,7)(5,8),(1,7)(2,5)(3,4)$

$(6,8),()]), \operatorname{Group}([(1,5)(2,7)(3,6)(4,8),(1,6)(2,4)(3,5)(7,8),()])]$

gap> Length (GsHNPtrueMin);

14

gap> List (GsHNPtrueMin, IdSmallGroup);

$[[8,2],[4,2],[4,2],[8,2],[4,2],[4,2],[8,2]$,

$[8,2],[8,2],[8,2],[4,2],[4,2],[4,2],[4,2]]$

gap $>$ Collected (List (GsHNPfalse, $x->$ Filtered (GsHNPtrueMin, $y->$ IsSubgroup $(x, y)$ ))) ;

$[[[], 81]]$

gap> Gs:=AllSubgroups (G); ;

gap> Length(Gs);

110 


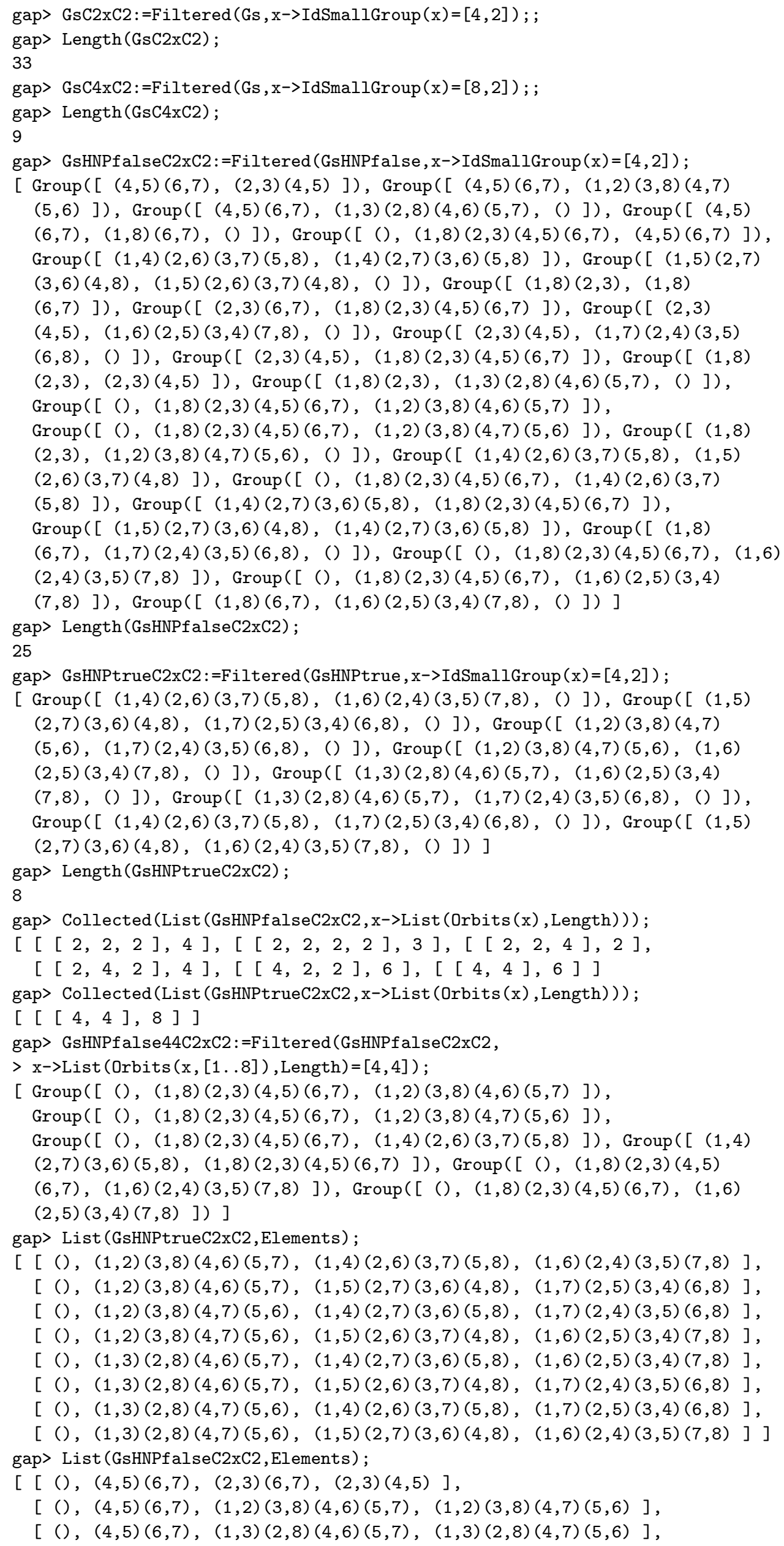


$[(),(4,5)(6,7),(1,8)(6,7),(1,8)(4,5)]$,

$[(),(4,5)(6,7),(1,8)(2,3),(1,8)(2,3)(4,5)(6,7)]$,

$[(),(2,3)(6,7),(1,4)(2,6)(3,7)(5,8),(1,4)(2,7)(3,6)(5,8)]$,

$[(),(2,3)(6,7),(1,5)(2,6)(3,7)(4,8),(1,5)(2,7)(3,6)(4,8)]$,

$[(),(2,3)(6,7),(1,8)(6,7),(1,8)(2,3)]$,

$[(),(2,3)(6,7),(1,8)(4,5),(1,8)(2,3)(4,5)(6,7)]$,

$[(),(2,3)(4,5),(1,6)(2,4)(3,5)(7,8),(1,6)(2,5)(3,4)(7,8)]$,

$[(),(2,3)(4,5),(1,7)(2,4)(3,5)(6,8),(1,7)(2,5)(3,4)(6,8)]$,

$[(),(2,3)(4,5),(1,8)(6,7),(1,8)(2,3)(4,5)(6,7)]$,

$[(),(2,3)(4,5),(1,8)(4,5),(1,8)(2,3)]$,

[ ()$,(1,2)(3,8)(4,6)(5,7),(1,3)(2,8)(4,6)(5,7),(1,8)(2,3)]$,

$[(),(1,2)(3,8)(4,6)(5,7),(1,3)(2,8)(4,7)(5,6),(1,8)(2,3)(4,5)(6,7)]$,

$[(),(1,2)(3,8)(4,7)(5,6),(1,3)(2,8)(4,6)(5,7),(1,8)(2,3)(4,5)(6,7)]$,

$[(),(1,2)(3,8)(4,7)(5,6),(1,3)(2,8)(4,7)(5,6),(1,8)(2,3)]$,

$[(),(1,4)(2,6)(3,7)(5,8),(1,5)(2,6)(3,7)(4,8),(1,8)(4,5)]$,

$[(),(1,4)(2,6)(3,7)(5,8),(1,5)(2,7)(3,6)(4,8),(1,8)(2,3)(4,5)(6,7)]$,

$[(),(1,4)(2,7)(3,6)(5,8),(1,5)(2,6)(3,7)(4,8),(1,8)(2,3)(4,5)(6,7)]$,

$[(),(1,4)(2,7)(3,6)(5,8),(1,5)(2,7)(3,6)(4,8),(1,8)(4,5)]$,

$[(),(1,6)(2,4)(3,5)(7,8),(1,7)(2,4)(3,5)(6,8),(1,8)(6,7)]$,

$[(),(1,6)(2,4)(3,5)(7,8),(1,7)(2,5)(3,4)(6,8),(1,8)(2,3)(4,5)(6,7)]$,

$[(),(1,6)(2,5)(3,4)(7,8),(1,7)(2,4)(3,5)(6,8),(1,8)(2,3)(4,5)(6,7)]$,

$[(),(1,6)(2,5)(3,4)(7,8),(1,7)(2,5)(3,4)(6,8),(1,8)(6,7)]]$

gap> List (GsHNPfalse44C2xC2, Elements) ;

$[[(),(1,2)(3,8)(4,6)(5,7),(1,3)(2,8)(4,7)(5,6),(1,8)(2,3)(4,5)(6,7)]$,

$[(),(1,2)(3,8)(4,7)(5,6),(1,3)(2,8)(4,6)(5,7),(1,8)(2,3)(4,5)(6,7)]$,

$[(),(1,4)(2,6)(3,7)(5,8),(1,5)(2,7)(3,6)(4,8),(1,8)(2,3)(4,5)(6,7)]$,

$[(),(1,4)(2,7)(3,6)(5,8),(1,5)(2,6)(3,7)(4,8),(1,8)(2,3)(4,5)(6,7)]$,

$[(),(1,6)(2,4)(3,5)(7,8),(1,7)(2,5)(3,4)(6,8),(1,8)(2,3)(4,5)(6,7)]$,

$[(),(1,6)(2,5)(3,4)(7,8),(1,7)(2,4)(3,5)(6,8),(1,8)(2,3)(4,5)(6,7)]]$

gap> ZG:=Centre $(G)$;

$\operatorname{Group}([(1,8)(2,3)(4,5)(6,7)])$

gap> List (GsHNPtrueC2xC2, $\mathrm{x}->$ Intersection $(\mathrm{x}, \mathrm{ZG}))$;

$[\operatorname{Group}(()), \operatorname{Group}(()), \operatorname{Group}(()), \operatorname{Group}(()), \operatorname{Group}(()), \operatorname{Group}(())$, $\operatorname{Group}(()), \operatorname{Group}(())]$

gap> List (GsHNPfalse44C2xC2, $\mathrm{x}->$ Intersection $(\mathrm{x}, \mathrm{ZG})$ ) ;

$[\operatorname{Group}([(1,8)(2,3)(4,5)(6,7)]), \operatorname{Group}([(1,8)(2,3)(4,5)(6,7)])$,

$\operatorname{Group}([(1,8)(2,3)(4,5)(6,7)]), \operatorname{Group}([(1,8)(2,3)(4,5)(6,7)])$,

$\operatorname{Group}([(1,8)(2,3)(4,5)(6,7)]), \operatorname{Group}([(1,8)(2,3)(4,5)(6,7)])]$

gap> GsHNPfalseC4xC2: $=$ Filtered (GsHNPfalse, $x->\operatorname{IdSmallGroup}(\mathrm{x})=[8,2])$;

[ $\operatorname{Group}([(),(1,8)(2,3)(4,5)(6,7),(4,5)(6,7),(1,2,8,3)(4,6,5,7)])$,

$\operatorname{Group}([(2,3)(6,7),(1,4,8,5)(2,6,3,7),(1,8)(2,3)(4,5)(6,7)])$,

$\operatorname{Group}([(),(1,8)(2,3)(4,5)(6,7),(1,6,8,7)(2,5,3,4),(2,3)(4,5)])]$

gap> Length(GsHNPfalseC4xC2);

3

gap> GsHNPtrueC4xC2:=Filtered (GsHNPtrue, $\mathrm{x}->\operatorname{IdSmallGroup}(\mathrm{x})=[8,2]$ ) ;

$[\operatorname{Group}([(),(1,8)(2,3)(4,5)(6,7),(1,2)(3,8)(4,6)(5,7),(1,4,8,5)$

$(2,6,3,7)]), \operatorname{Group}([(),(1,8)(2,3)(4,5)(6,7),(1,6,8,7)(2,5,3,4),(1,2)$

$(3,8)(4,7)(5,6)]), \operatorname{Group}([(),(1,8)(2,3)(4,5)(6,7),(1,4)(2,6)(3,7)$

$(5,8),(1,2,8,3)(4,6,5,7)]), \operatorname{Group}([(),(1,8)(2,3)(4,5)(6,7),(1,4,8,5)$

$(2,6,3,7),(1,2,8,3)(4,6,5,7)]), \operatorname{Group}([(),(1,8)(2,3)(4,5)$

$(6,7),(1,2,8,3)(4,7,5,6),(1,4)(2,7)(3,6)(5,8)]), \operatorname{Group}([(),(1,8)(2,3)$

$(4,5)(6,7),(1,2,8,3)(4,7,5,6),(1,6)(2,4)(3,5)(7,8)])]$

gap> Length (GsHNPtrueC4xC2);

6

gap> Collected (List(GsHNPfalseC4xC2, x->List(Orbits (x), Length))) ;

$[[[4,4], 3]]$

gap> Collected(List (GsHNPtrueC4xC2, $\mathrm{x}->$ List (Orbits (x), Length))) ;

$[[$ [ $], 6]]$

$(2-6) G^{\prime}=8 T 32 \simeq\left(\left(C_{2}\right)^{3} \rtimes V_{4}\right) \rtimes C_{3}$.

gap> Read("HNP.gap") ;

gap> G:=TransitiveGroup $(8,32) ; \# \mathrm{G}=8 \mathrm{~T} 32=(\mathrm{C} 2 \sim 3: \mathrm{V} 4): \mathrm{C} 3$

$[2 \sim 3] \mathrm{A}(4)$ 


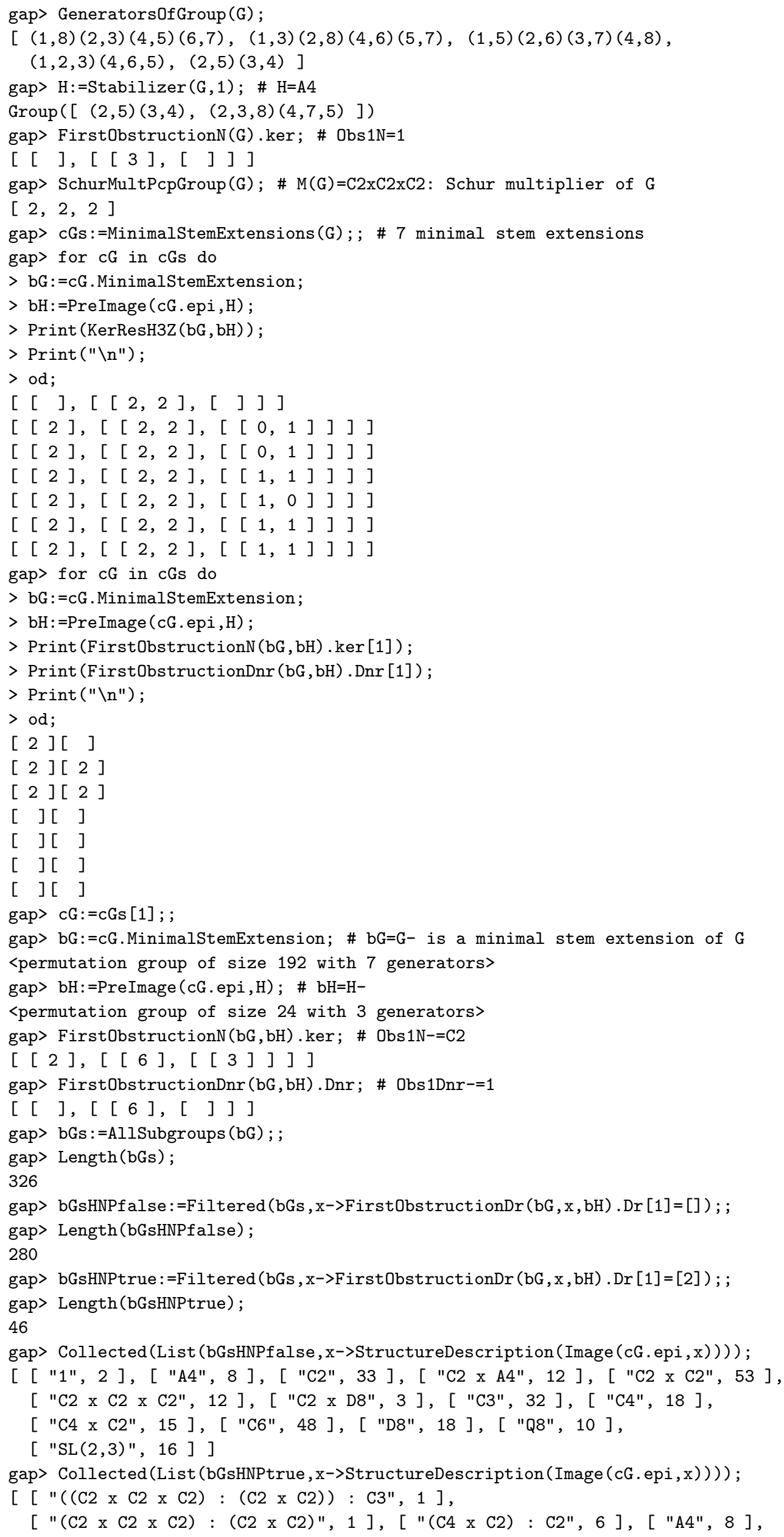




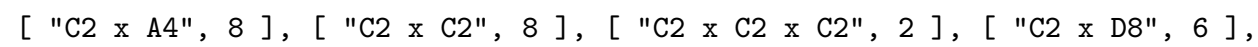

[ "C4 x C2", 6 ] ]

gap> GsHNPfalse:=Set (bGsHNPfalse, $\mathrm{x}->\operatorname{Image}(\mathrm{cG}$. epi, $\mathrm{x})$ ); ;

gap> Length(GsHNPfalse);

129

gap> GsHNPtrue:=Set (bGsHNPtrue, $\mathrm{x}->\operatorname{Image}(\mathrm{cG} . \mathrm{ep} \mathrm{i}, \mathrm{x}))$; ;

gap> Length (GsHNPtrue);

46

gap> Intersection(GsHNPfalse, GsHNPtrue);

[ ]

gap> GsHNPtrueMin:=Filtered(GsHNPtrue, $\mathrm{x}->$ Length(Filtered (GsHNPtrue,

$>\mathrm{y}->\operatorname{IsSubgroup}(\mathrm{x}, \mathrm{y})))=1)$;

[ $\operatorname{Group}([(1,7)(2,3)(4,5)(6,8),(1,2)(3,7)(4,8)(5,6),()]), \operatorname{Group}([(1,3,6,4)$

$(2,7,5,8),(1,8,6,7)(2,4,5,3),(1,6)(2,5)(3,4)(7,8),(1,6)(2,5)(3,4)$

$(7,8)]), \operatorname{Group}([(1,4)(2,8)(3,6)(5,7),(1,8)(2,4)(3,5)(6,7),()])$,

$\operatorname{Group}([(1,8)(2,3)(4,5)(6,7),(1,2)(3,8)(4,7)(5,6),()]), \operatorname{Group}([(1,7,6,8)$

$(2,4,5,3),(1,2)(3,8)(4,7)(5,6),(1,6)(2,5)(3,4)(7,8),(1,6)(2,5)(3,4)$

$(7,8)]), \operatorname{Group}([(1,4)(2,7)(3,6)(5,8),(1,7)(2,4)(3,5)(6,8),()])$,

Group $([(1,7,6,8)(2,4,5,3),(1,2,6,5)(3,7,4,8),(1,6)(2,5)(3,4)(7,8),(1,6)$

$(2,5)(3,4)(7,8)]), \operatorname{Group}([(1,3,6,4)(2,7,5,8),(1,7)(2,4)(3,5)(6,8),(1,6)$

$(2,5)(3,4)(7,8),(1,6)(2,5)(3,4)(7,8)]), \operatorname{Group}([(1,5,6,2)(3,7,4,8),(1,3)$

$(2,8)(4,6)(5,7),(1,6)(2,5)(3,4)(7,8),(1,6)(2,5)(3,4)(7,8)])$,

Group $([(1,5,6,2)(3,7,4,8),(1,3,6,4)(2,8,5,7),(1,6)(2,5)(3,4)(7,8),(1,6)$

$(2,5)(3,4)(7,8)]), \operatorname{Group}([(1,8)(2,4)(3,5)(6,7),(1,5)(2,6)(3,8)$

$(4,7),()]), \operatorname{Group}([(1,7)(2,4)(3,5)(6,8),(1,5)(2,6)(3,7)(4,8),()])$, $\operatorname{Group}([(1,8)(2,3)(4,5)(6,7),(1,5)(2,6)(3,7)(4,8),()]), \operatorname{Group}([(1,7)$

$(2,3)(4,5)(6,8),(1,5)(2,6)(3,8)(4,7),()])]$

gap> Length(GsHNPtrueMin);

14

gap> List (GsHNPtrueMin, IdSmallGroup) ;

$[[4,2],[8,2],[4,2],[4,2],[8,2],[4,2],[8,2]$,

$[8,2],[8,2],[8,2],[4,2],[4,2],[4,2],[4,2]]$

gap $>$ Collected (List (GsHNPfalse, $\mathrm{x}->$ Filtered (GsHNPtrueMin, $y->\operatorname{IsSubgroup}(\mathrm{x}, \mathrm{y})$ ))) ;

[ [ [ ], 129 ] ]

gap> Gs:=AllSubgroups $(G) ;$;

gap> Length(Gs);

175

gap> GsC2xC2:=Filtered (Gs, $\mathrm{x}->\operatorname{IdSmallGroup}(\mathrm{x})=[4,2])$; ;

gap> Length (GsC2xC2);

33

gap> GsC4xC2:=Filtered(Gs, $\mathrm{x}->\operatorname{IdSmallGroup}(\mathrm{x})=[8,2])$; ;

gap> Length (GsC4xC2);

9

gap> GsHNPfalseC2xC2: $=$ Filtered (GsHNPfalse, $\mathrm{x}->\operatorname{IdSmallGroup}(\mathrm{x})=[4,2])$;

$[\operatorname{Group}([(3,4)(7,8),(2,5)(7,8)]), \operatorname{Group}([(1,2)(3,8)(4,7)(5,6),(3,4)$

$(7,8),(3,4)(7,8)]), \operatorname{Group}([(1,5)(2,6)(3,7)(4,8),(3,4)(7,8),(3,4)$

$(7,8)]), \operatorname{Group}([(1,6)(3,4),(3,4)(7,8),(1,6)(7,8)]), \operatorname{Group}([(3,4)$

$(7,8),(1,6)(2,5)(3,4)(7,8)]), \operatorname{Group}([(1,3)(2,8)(4,6)(5,7),(2,5)$

$(7,8),(2,5)(7,8)]), \operatorname{Group}([(1,4)(2,7)(3,6)(5,8),(2,5)(7,8),(2,5)$

$(7,8)]), \operatorname{Group}([(2,5)(7,8),(1,6)(2,5),(1,6)(7,8)]), \operatorname{Group}([(1,6)$

$(3,4),(1,6)(2,5)(3,4)(7,8)]), \operatorname{Group}([(1,6)(2,5)(3,4)(7,8),(2,5)$

$(3,4),(1,6)(7,8)]), \operatorname{Group}([(2,5)(3,4),(1,6)(2,5)]), \operatorname{Group}([(1,7)(2,4)$

$(3,5)(6,8),(2,5)(3,4),(2,5)(3,4)]), \operatorname{Group}([(1,8)(2,3)(4,5)(6,7),(2,5)$

$(3,4),(2,5)(3,4)]), \operatorname{Group}([(1,5)(2,6)(3,7)(4,8),(1,6)(2,5),(1,6)$

$(2,5)]), \operatorname{Group}([(1,5)(2,6)(3,8)(4,7),(1,6)(2,5)(3,4)(7,8),(1,6)(2,5)$

$(3,4)(7,8)]), \operatorname{Group}([(1,2)(3,8)(4,7)(5,6),(1,6)(2,5)(3,4)(7,8),(1,6)$

$(2,5)(3,4)(7,8)]), \operatorname{Group}([(1,2)(3,8)(4,7)(5,6),(1,6)(2,5),(1,6)$

$(2,5)]), \operatorname{Group}([(1,4)(2,7)(3,6)(5,8),(1,6)(3,4),(1,6)(3,4)])$,

Group $([(1,3)(2,7)(4,6)(5,8),(1,6)(2,5)(3,4)(7,8),(1,6)(2,5)(3,4)$

$(7,8)]), \operatorname{Group}([(1,3)(2,8)(4,6)(5,7),(1,6)(2,5)(3,4)(7,8),(1,6)(2,5)$

$(3,4)(7,8)]), \operatorname{Group}([(1,3)(2,8)(4,6)(5,7),(1,6)(3,4),(1,6)(3,4)])$,

$\operatorname{Group}([(1,8)(2,3)(4,5)(6,7),(1,6)(7,8),(1,6)(7,8)]), \operatorname{Group}([(1,7)(2,4)$

$(3,5)(6,8),(1,6)(7,8),(1,6)(7,8)]), \operatorname{Group}([(1,8)(2,4)(3,5)(6,7),(1,6)$ 
$(2,5)(3,4)(7,8),(1,6)(2,5)(3,4)(7,8)]), \operatorname{Group}([(1,7)(2,4)(3,5)$

$(6,8),(1,6)(2,5)(3,4)(7,8),(1,6)(2,5)(3,4)(7,8)])]$

gap> Length (GsHNPfalseC2xC2);

25

gap> GsHNPtrueC2xC2:=Filtered(GsHNPtrue, $\mathrm{x}->\operatorname{IdSmallGroup}(\mathrm{x})=[4,2])$;

$[\operatorname{Group}([(1,7)(2,3)(4,5)(6,8),(1,2)(3,7)(4,8)(5,6),()]), \operatorname{Group}([(1,4)$

$(2,8)(3,6)(5,7),(1,8)(2,4)(3,5)(6,7),()]), \operatorname{Group}([(1,8)(2,3)(4,5)$

$(6,7),(1,2)(3,8)(4,7)(5,6),()]), \operatorname{Group}([(1,4)(2,7)(3,6)(5,8),(1,7)(2,4)$

$(3,5)(6,8),()]), \operatorname{Group}([(1,8)(2,4)(3,5)(6,7),(1,5)(2,6)(3,8)$

$(4,7),()]), \operatorname{Group}([(1,7)(2,4)(3,5)(6,8),(1,5)(2,6)(3,7)(4,8),()])$, $\operatorname{Group}([(1,8)(2,3)(4,5)(6,7),(1,5)(2,6)(3,7)(4,8),()]), \operatorname{Group}([(1,7)$

$(2,3)(4,5)(6,8),(1,5)(2,6)(3,8)(4,7),()])]$

gap> Length (GsHNPtrueC2xC2);

8

gap> Collected (List (GsHNPfalseC2xC2, x->List(Orbits (x), Length)));

$[[[2,2,2], 4],[[2,2,2,2], 3],[[2,2,4], 1]$,

$[[2,4,2], 5],[[4,2,2], 6],[[4,4], 6]]$

gap> Collected(List (GsHNPtrueC2xC2, x->List (Orbits (x), Length)));

$[[[4,4], 8]]$

gap> GsHNPfalse44C2xC2:=Filtered(GsHNPfalseC2xC2,

$>\mathrm{x}->\operatorname{List}(\operatorname{Orbits}(\mathrm{x},[1 \ldots 8])$, Length $)=[4,4])$;

$[\operatorname{Group}([(1,5)(2,6)(3,8)(4,7),(1,6)(2,5)(3,4)(7,8),(1,6)(2,5)(3,4)$

$(7,8)]), \operatorname{Group}([(1,2)(3,8)(4,7)(5,6),(1,6)(2,5)(3,4)(7,8),(1,6)(2,5)$

$(3,4)(7,8)]), \operatorname{Group}([(1,3)(2,7)(4,6)(5,8),(1,6)(2,5)(3,4)(7,8),(1,6)$

$(2,5)(3,4)(7,8)]), \operatorname{Group}([(1,3)(2,8)(4,6)(5,7),(1,6)(2,5)(3,4)$

$(7,8),(1,6)(2,5)(3,4)(7,8)]), \operatorname{Group}([(1,8)(2,4)(3,5)(6,7),(1,6)(2,5)$

$(3,4)(7,8),(1,6)(2,5)(3,4)(7,8)]), \operatorname{Group}([(1,7)(2,4)(3,5)(6,8),(1,6)$

$(2,5)(3,4)(7,8),(1,6)(2,5)(3,4)(7,8)])]$

gap> List (GsHNPtrueC2xC2, Elements);

$[[(),(1,2)(3,7)(4,8)(5,6),(1,3)(2,7)(4,6)(5,8),(1,7)(2,3)(4,5)(6,8)]$,

$[(),(1,2)(3,7)(4,8)(5,6),(1,4)(2,8)(3,6)(5,7),(1,8)(2,4)(3,5)(6,7)]$,

$[(),(1,2)(3,8)(4,7)(5,6),(1,3)(2,8)(4,6)(5,7),(1,8)(2,3)(4,5)(6,7)]$,

$[(),(1,2)(3,8)(4,7)(5,6),(1,4)(2,7)(3,6)(5,8),(1,7)(2,4)(3,5)(6,8)]$,

$[(),(1,3)(2,7)(4,6)(5,8),(1,5)(2,6)(3,8)(4,7),(1,8)(2,4)(3,5)(6,7)]$,

$[(),(1,3)(2,8)(4,6)(5,7),(1,5)(2,6)(3,7)(4,8),(1,7)(2,4)(3,5)(6,8)]$,

$[(),(1,4)(2,7)(3,6)(5,8),(1,5)(2,6)(3,7)(4,8),(1,8)(2,3)(4,5)(6,7)]$,

$[(),(1,4)(2,8)(3,6)(5,7),(1,5)(2,6)(3,8)(4,7),(1,7)(2,3)(4,5)(6,8)]]$ gap> List (GsHNPfalseC2xC2, Elements);

$[[(),(3,4)(7,8),(2,5)(7,8),(2,5)(3,4)]$,

$[(),(3,4)(7,8),(1,2)(3,7)(4,8)(5,6),(1,2)(3,8)(4,7)(5,6)]$,

$[(),(3,4)(7,8),(1,5)(2,6)(3,7)(4,8),(1,5)(2,6)(3,8)(4,7)]$,

$[(),(3,4)(7,8),(1,6)(7,8),(1,6)(3,4)]$,

$[(),(3,4)(7,8),(1,6)(2,5),(1,6)(2,5)(3,4)(7,8)]$,

$[(),(2,5)(7,8),(1,3)(2,7)(4,6)(5,8),(1,3)(2,8)(4,6)(5,7)]$,

$[(),(2,5)(7,8),(1,4)(2,7)(3,6)(5,8),(1,4)(2,8)(3,6)(5,7)]$,

$[(),(2,5)(7,8),(1,6)(7,8),(1,6)(2,5)]$,

$[(),(2,5)(7,8),(1,6)(3,4),(1,6)(2,5)(3,4)(7,8)]$,

$[(),(2,5)(3,4),(1,6)(7,8),(1,6)(2,5)(3,4)(7,8)]$,

$[(),(2,5)(3,4),(1,6)(3,4),(1,6)(2,5)]$,

$[(),(2,5)(3,4),(1,7)(2,3)(4,5)(6,8),(1,7)(2,4)(3,5)(6,8)]$,

$[(),(2,5)(3,4),(1,8)(2,3)(4,5)(6,7),(1,8)(2,4)(3,5)(6,7)]$,

$[(),(1,2)(3,7)(4,8)(5,6),(1,5)(2,6)(3,7)(4,8),(1,6)(2,5)]$,

$[(),(1,2)(3,7)(4,8)(5,6),(1,5)(2,6)(3,8)(4,7),(1,6)(2,5)(3,4)(7,8)]$,

$[(),(1,2)(3,8)(4,7)(5,6),(1,5)(2,6)(3,7)(4,8),(1,6)(2,5)(3,4)(7,8)]$,

$[(),(1,2)(3,8)(4,7)(5,6),(1,5)(2,6)(3,8)(4,7),(1,6)(2,5)]$,

$[(),(1,3)(2,7)(4,6)(5,8),(1,4)(2,7)(3,6)(5,8),(1,6)(3,4)]$,

$[(),(1,3)(2,7)(4,6)(5,8),(1,4)(2,8)(3,6)(5,7),(1,6)(2,5)(3,4)(7,8)]$,

$[(),(1,3)(2,8)(4,6)(5,7),(1,4)(2,7)(3,6)(5,8),(1,6)(2,5)(3,4)(7,8)]$,

$[(),(1,3)(2,8)(4,6)(5,7),(1,4)(2,8)(3,6)(5,7),(1,6)(3,4)]$,

$[(),(1,6)(7,8),(1,7)(2,3)(4,5)(6,8),(1,8)(2,3)(4,5)(6,7)]$,

$[(),(1,6)(7,8),(1,7)(2,4)(3,5)(6,8),(1,8)(2,4)(3,5)(6,7)]$,

$[(),(1,6)(2,5)(3,4)(7,8),(1,7)(2,3)(4,5)(6,8),(1,8)(2,4)(3,5)(6,7)]$,

$[(),(1,6)(2,5)(3,4)(7,8),(1,7)(2,4)(3,5)(6,8),(1,8)(2,3)(4,5)(6,7)]]$ 
gap> List (GsHNPfalse44C2xC2, Elements) ;

$[[(),(1,2)(3,7)(4,8)(5,6),(1,5)(2,6)(3,8)(4,7),(1,6)(2,5)(3,4)(7,8)]$, $[(),(1,2)(3,8)(4,7)(5,6),(1,5)(2,6)(3,7)(4,8),(1,6)(2,5)(3,4)(7,8)]$,

$[(),(1,3)(2,7)(4,6)(5,8),(1,4)(2,8)(3,6)(5,7),(1,6)(2,5)(3,4)(7,8)]$,

$[(),(1,3)(2,8)(4,6)(5,7),(1,4)(2,7)(3,6)(5,8),(1,6)(2,5)(3,4)(7,8)]$,

$[(),(1,6)(2,5)(3,4)(7,8),(1,7)(2,3)(4,5)(6,8),(1,8)(2,4)(3,5)(6,7)]$,

$[(),(1,6)(2,5)(3,4)(7,8),(1,7)(2,4)(3,5)(6,8),(1,8)(2,3)(4,5)(6,7)]]$

gap> ZG:=Centre $(G)$;

$\operatorname{Group}([(1,6)(2,5)(3,4)(7,8)])$

gap> List (GsHNPtrueC2xC2, $\mathrm{x}->$ Intersection $(\mathrm{x}, \mathrm{ZG}))$;

$[\operatorname{Group}(()), \operatorname{Group}(()), \operatorname{Group}(()), \operatorname{Group}(()), \operatorname{Group}(()), \operatorname{Group}(())$,

$\operatorname{Group}(()), \operatorname{Group}(())]$

gap> List (GsHNPfalse44C2xC2, $\mathrm{x}->$ Intersection $(\mathrm{x}, \mathrm{ZG}))$;

$[\operatorname{Group}([(1,6)(2,5)(3,4)(7,8)]), \operatorname{Group}([(1,6)(2,5)(3,4)(7,8)])$, $\operatorname{Group}([(1,6)(2,5)(3,4)(7,8)]), \operatorname{Group}([(1,6)(2,5)(3,4)(7,8)])$, $\operatorname{Group}([(1,6)(2,5)(3,4)(7,8)]), \operatorname{Group}([(1,6)(2,5)(3,4)(7,8)])]$

gap> GsHNPfalseC4xC2: =Filtered (GsHNPfalse, $\mathrm{x}->\operatorname{IdSmallGroup}(\mathrm{x})=[8,2])$;

$[\operatorname{Group}([(1,2,6,5)(3,8,4,7),(1,6)(2,5)(3,4)(7,8),(3,4)(7,8)])$,

$\operatorname{Group}([(1,3,6,4)(2,7,5,8),(1,6)(2,5)(3,4)(7,8),(1,6)(3,4),(2,5)$

$(7,8)]), \operatorname{Group}([(1,7,6,8)(2,4,5,3),(1,6)(2,5)(3,4)(7,8),(2,5)(3,4)])]$ gap> Length (GsHNPfalseC4xC2);

3

gap> GsHNPtrueC4xC2: $=$ Filtered (GsHNPtrue, $\mathrm{x}->\operatorname{IdSmallGroup}(\mathrm{x})=[8,2])$;

$[\operatorname{Group}([(1,3,6,4)(2,7,5,8),(1,8,6,7)(2,4,5,3),(1,6)(2,5)(3,4)(7,8),(1,6)$

$(2,5)(3,4)(7,8)]), \operatorname{Group}([(1,7,6,8)(2,4,5,3),(1,2)(3,8)(4,7)(5,6),(1,6)$

$(2,5)(3,4)(7,8),(1,6)(2,5)(3,4)(7,8)]), \operatorname{Group}([(1,7,6,8)(2,4,5,3),(1,2$, $6,5)(3,7,4,8),(1,6)(2,5)(3,4)(7,8),(1,6)(2,5)(3,4)(7,8)])$,

Group $([(1,3,6,4)(2,7,5,8),(1,7)(2,4)(3,5)(6,8),(1,6)(2,5)(3,4)$

$(7,8),(1,6)(2,5)(3,4)(7,8)]), \operatorname{Group}([(1,5,6,2)(3,7,4,8),(1,3)(2,8)(4,6)$

$(5,7),(1,6)(2,5)(3,4)(7,8),(1,6)(2,5)(3,4)(7,8)]), \operatorname{Group}([(1,5,6,2)$

$(3,7,4,8),(1,3,6,4)(2,8,5,7),(1,6)(2,5)(3,4)(7,8),(1,6)(2,5)(3,4)$

$(7,8)$ ] ] ]

gap> Length(GsHNPtrueC4xC2);

6

gap> Collected (List (GsHNPfalseC4xC2, x->List (Orbits (x), Length)));

$[[4,4], 3]]$

gap> Collected(List(GsHNPtrueC4xC2, $\mathrm{x}->$ List (Orbits (x), Length))) ;

$[[[8], 6]]$

gap> Syl2G:=SylowSubgroup $(G, 2)$;

$\operatorname{Group}([(2,5)(3,4),(2,5)(7,8),(1,2)(3,8)(4,7)(5,6),(1,8)(2,3)(4,5)$

$(6,7),(1,6)(2,5)(3,4)(7,8)])$

gap> IsNormal (G, Syl2G);

true

gap> IsConjugate(SymmetricGroup (8), $\operatorname{Syl2G,TransitiveGroup~}(8,22))$;

true

Example 6.13 $(G=9 T m(m=2,5,7,9,11,14,23))$.

$(3-1) G=9 T 2 \simeq\left(C_{3}\right)^{2}$.

gap> Read("HNP.gap");

gap> G:=TransitiveGroup $(9,2) ; \# \mathrm{G}=9 \mathrm{~T} 2=\mathrm{C} 3 \mathrm{xC} 3$

$E(9)=3[x] 3$

gap> $\mathrm{H}:=\operatorname{Stabilizer}(\mathrm{G}, 1) ; \quad \# \mathrm{H}=1$

$\operatorname{Group}(())$

gap> FirstObstructionN(G).ker; \# Obs1N=1

[ [ ], [ [ ], [ ] ] ]

gap> SchurMultPcpGroup $(G)$; \# $M(G)=C 3$ : Schur multiplier of $G$

[ 3 ]

gap> ScG:=SchurCoverG $(G)$;

$\operatorname{rec}(\operatorname{SchurCover}:=\operatorname{Group}([(2,4,5)(3,6,7),(1,2,3)(4,7,8)(5,6,9)])$,

epi $:=[(2,4,5)(3,6,7),(1,2,3)(4,7,8)(5,6,9)] \rightarrow$

$[(1,4,7)(2,5,8)(3,6,9),(1,2,9)(3,4,5)(6,7,8)]$, Tid $:=[9,7])$

gap> StructureDescription(TransitiveGroup $(9,7))$; 


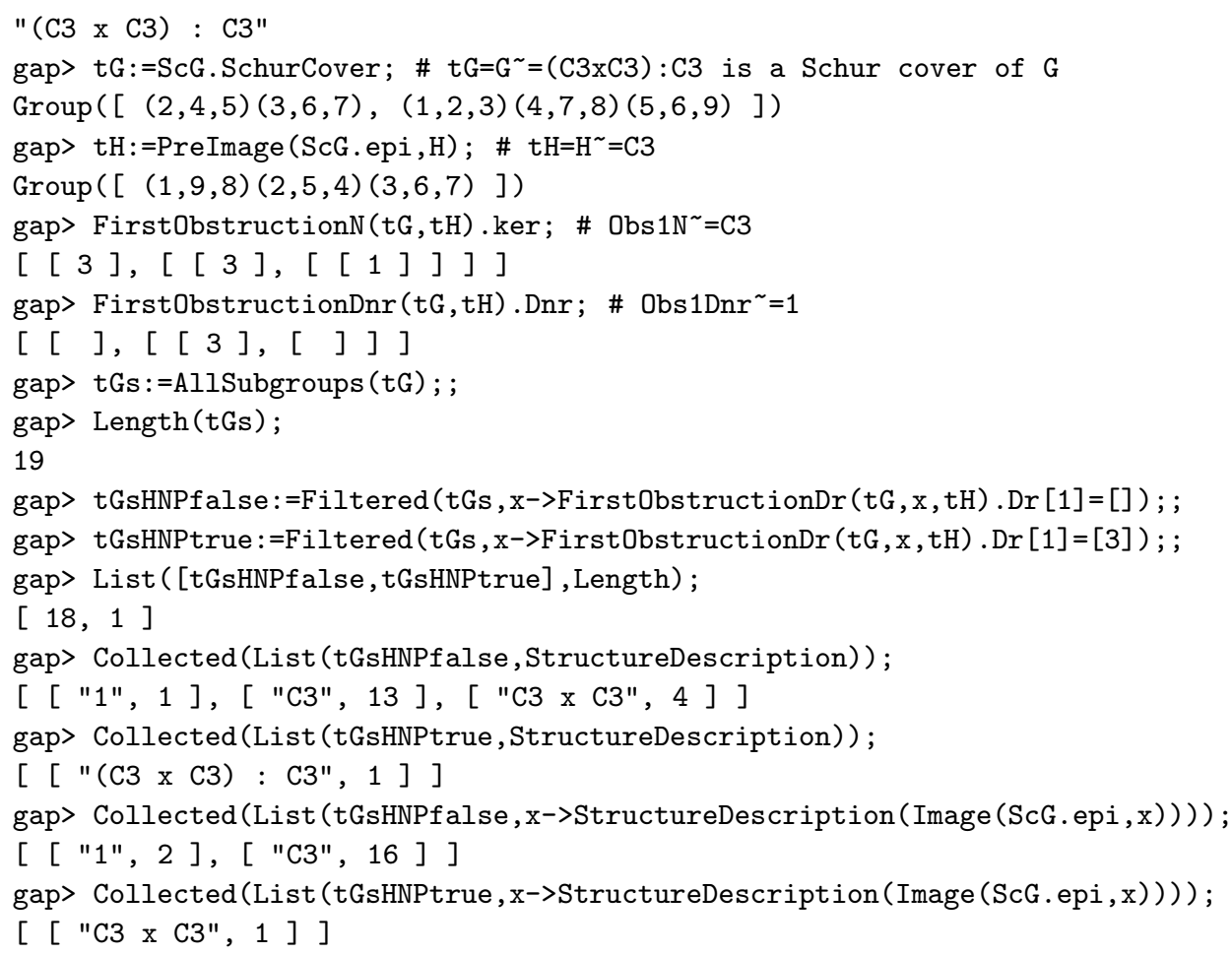


[ [ "1", 2 ], [ "C2", 18 ], [ "C3", 16 ], [ "S3", 24 ] ]

gap> Collected (List (tGsHNPtrue, $\mathrm{x}->$ StructureDescription (Image (ScG.epi, $\mathrm{x}$ )))) ;

$[$ [ "(C3 x C3) : C2", 1$],[$ "C3 x C3", 1$]$ ]

$(3-3) G=9 T 7 \simeq\left(C_{3}\right)^{2} \rtimes C_{3}$.

gap> Read("HNP.gap");

gap> G:=TransitiveGroup $(9,7) ; \# \mathrm{G}=9 \mathrm{~T} 7=(\mathrm{C} 3 \times \mathrm{C} 3): \mathrm{C} 3$

$E(9): 3=\left[3^{\wedge} 2\right] 3$

gap> $\mathrm{H}:=$ Stabilizer $(\mathrm{G}, 1) ; \# \mathrm{H}=\mathrm{C} 3$

$\operatorname{Group}([(3,4,5)(6,8,7)])$

gap> FirstObstructionN(G).ker; \# Obs1N=1

[ [ ], [ [ 3$],[$ ] ] ]

gap> SchurMultPcpGroup $(G) ; \# M(G)=C 3 \times C 3$ : Schur multiplier of $G$

$[3,3]$

gap> cGs:=MinimalStemExtensions $(G)$; ;

gap> for $c G$ in $c G s$ do

$>\mathrm{tG}:=\mathrm{cG}$.MinimalStemExtension;

$>\mathrm{tH}:=$ PreImage (cG.epi, $\mathrm{H})$;

$>\operatorname{Print}(\operatorname{KerResH} 3 \mathrm{Z}(\mathrm{tG}, \mathrm{tH}))$;

$>\operatorname{Print}(" \backslash \mathrm{n} ")$;

$>$ od;

$[[],[[3],[]]]$

$[[3],[[3],[[1]]]]$

$[[3],[[3,3],[[0,1]]]]$

$[[3],[[3,3],[[0,1]]]]$

gap> for $\mathrm{CG}$ in $\mathrm{cGs}$ do

$>\mathrm{bG}:=\mathrm{cG}$.MinimalStemExtension;

$>\mathrm{bH}:=$ PreImage (cG.epi, H) ;

$>$ Print (FirstObstructionN (bG,bH). $\operatorname{ker}[1]$ );

$>$ Print (FirstObstructionDnr (bG, bH).Dnr [1]);

$>\operatorname{Print}(" \backslash \mathrm{n} ")$;

$>$ od;

[ 3 ] [ ]

$\left[\begin{array}{ll}3 & ]\end{array}\right]$

[ 3 ] [ 3 ]

[ 3 ] [ 3 ]

gap> cG:=cGs [1];

rec( MinimalStemExtension := <permutation group of size 81 with 4 generators>, epi $:=[(1,5,15)(2,9,24)(3,12,29)(4,14,31)(6,18,37)(7,21,42)(8,23,44)(10$, $26,47)(11,28,49)(13,30,50)(16,34,55)(17,36,57)(19,39,60)(20,41,62)(22$, $43,63)(25,46,65)(27,48,66)(32,52,69)(33,54,71)(35,56,72)(38,59,74)(40$, $61,75)(45,64,76)(51,68,78)(53,70,79)(58,73,80)(67,77,81)$, $(1,2,6)(3,22,51)(4,40,32)(5,23,34)(7,35,25)(8,53,10)(9,36,12)(11,20$, 33) $(13,38,16)(14,21,18)(15,63,69)(17,27,19)(24,72,47)(26,43,77)(28,61$, 68) $(29,44,71)(30,73,52)(31,62,55)(37,50,60)(39,56,64)(41,70,46)(42,57$, 49) $(45,58,67)(48,59,54)(65,75,81)(66,80,78)(74,79,76)$, $(1,3,10)(2,7,19)(4,11,25)(5,12,26)(6,16,32)(8,20,38)(9,21,39)(13,27$, $45)(14,28,46)(15,29,47)(17,33,51)(18,34,52)(22,40,58)(23,41,59)(24,42$, $60)(30,48,64)(31,49,65)(35,53,67)(36,54,68)(37,55,69)(43,61,73)(44,62$, $74)(50,66,76)(56,70,77)(57,71,78)(63,75,80)(72,79,81)]$->

$[(3,4,5)(6,8,7),(1,4,7)(2,5,8)(3,6,9),(1,2,9)(3,4,5)(6,7,8)])$

gap> bG:=cG.MinimalStemExtension;

<permutation group of size 81 with 4 generators>

gap> bH:=PreImage (cG.epi,H); \# bH=H-=C3xC3

<permutation group of size 9 with 2 generators>

gap> KerResH3Z (bG, bH) ;

[ [ ], [ [ 3$],[$ ] ] ]

gap> FirstObstructionN (bG,bH).ker; \# Obs1N-=C3

[ [ 3$],[[3,3],[[0,1]]]]$

gap> FirstObstructionDnr (bG,bH).Dnr; \# Obs1Dnr-=1

$[[],[[3,3],[]]]$

gap> bGs:=AllSubgroups $(\mathrm{bG})$; ;

gap> Length(bGs); 


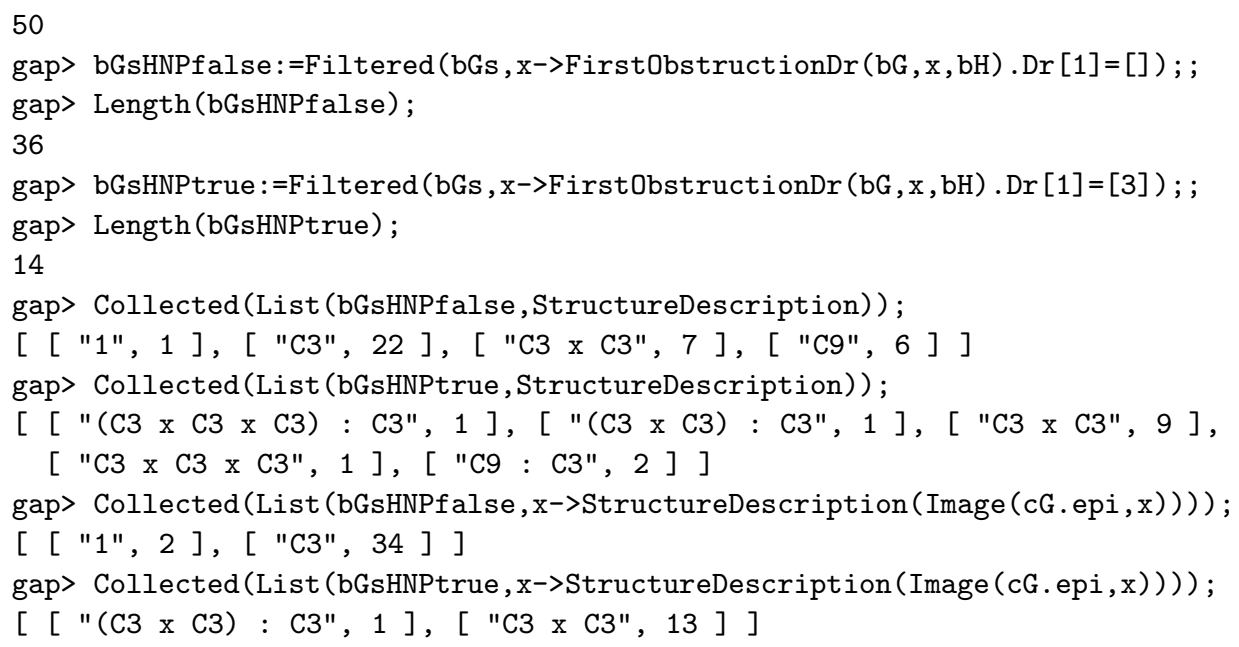


$(3-5) G=9 T 11 \simeq\left(C_{3}\right)^{2} \rtimes C_{6}$.

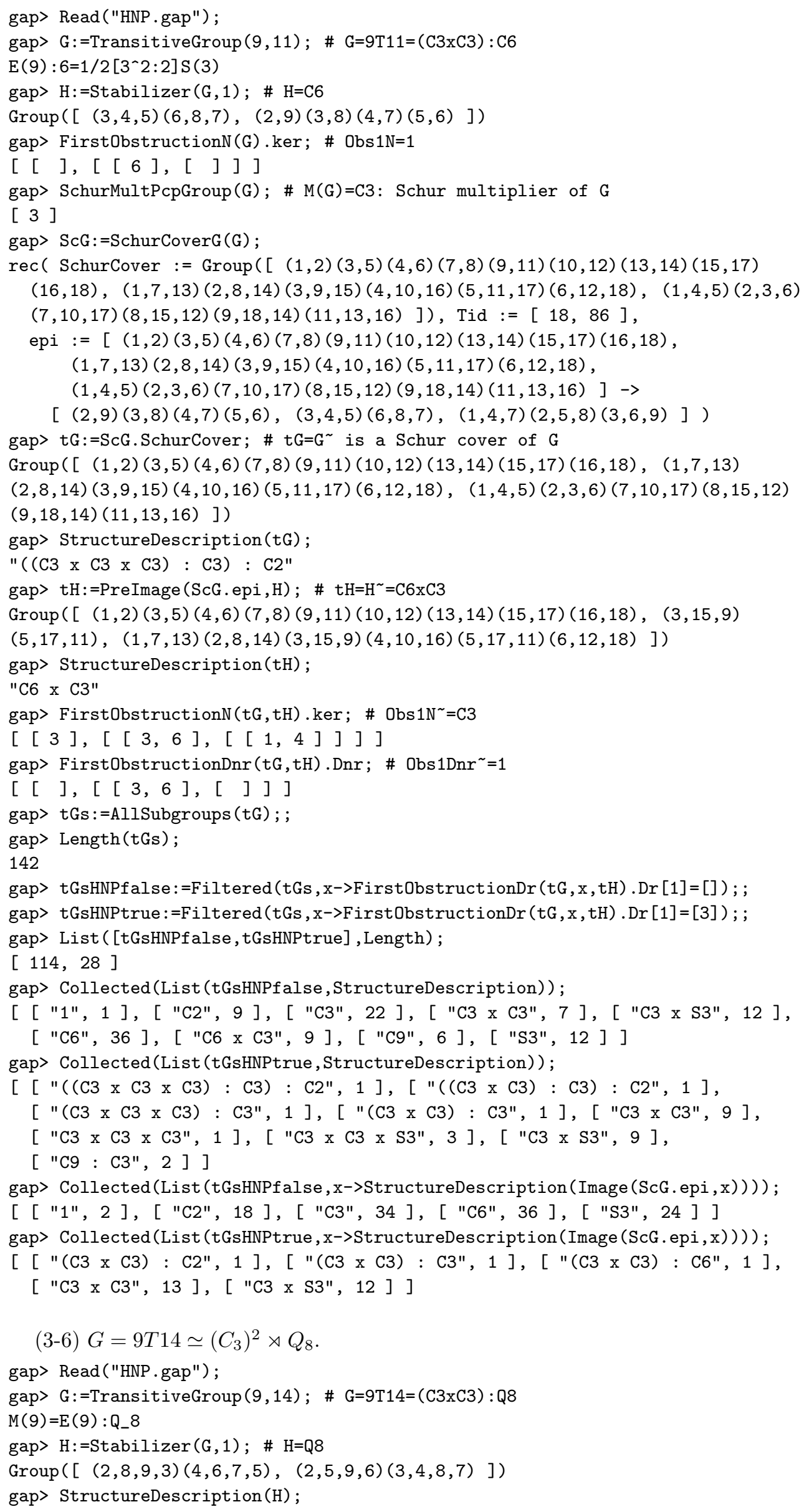


"Q8"

gap> FirstObstructionN(G).ker; \# Obs1N=1

$[$ [ ], [ [ 2, 2 ], [ ] ] ]

gap> SchurMultPcpGroup $(G)$; \# $M(G)=C 3$ : Schur multiplier of $G$

[ 3 ]

gap> ScG: $=$ SchurCoverG $(G)$;

rec( SchurCover := Group $([(2,4,3,5)(6,9,7,8)(10,20,13,19)(11,14,12,17)$

$(15,24,16,25)(18,22,21,23),(2,6,3,7)(4,8,5,9)(10,16,13,15)(11,22,12,23)$

$(14,18,17,21)(19,25,20,24),(1,2,3)(4,10,11)(5,12,13)(6,14,15)(7,16,17)$

$(8,18,19)(9,20,21)(22,23,26)(24,25,27)])$, Tid $:=[27,83]$,

epi $:=[(2,4,3,5)(6,9,7,8)(10,20,13,19)(11,14,12,17)(15,24,16,25)(18,22,21$, 23), $(2,6,3,7)(4,8,5,9)(10,16,13,15)(11,22,12,23)(14,18,17,21)(19,25$, $20,24),(1,2,3)(4,10,11)(5,12,13)(6,14,15)(7,16,17)(8,18,19)(9,20$, 21) $(22,23,26)(24,25,27)] \rightarrow$

$[(2,8,9,3)(4,6,7,5),(2,6,9,5)(3,7,8,4),(1,6,5)(2,7,3)(4,9,8)])$

gap> StructureDescription(TransitiveGroup $(27,83))$;

"((C3 x C3) : C3) : Q8"

gap> $t G:=S c G . S c h u r C o v e r ; \# t G=G^{\sim}$ is a Schur cover of $G$

Group $([(2,4,3,5)(6,9,7,8)(10,20,13,19)(11,14,12,17)(15,24,16,25)$

$(18,22,21,23),(2,6,3,7)(4,8,5,9)(10,16,13,15)(11,22,12,23)(14,18,17,21)$

$(19,25,20,24),(1,2,3)(4,10,11)(5,12,13)(6,14,15)(7,16,17)(8,18,19)(9,20,21)$

$(22,23,26)(24,25,27)])$

gap> $\mathrm{tH}:=$ PreImage $(\mathrm{ScG} . \mathrm{epi}, \mathrm{H}) ; \# \mathrm{tH}=\mathrm{H}^{\sim}=\mathrm{C} 3 \mathrm{xQ} 8$

$\operatorname{Group}([(2,4,3,5)(6,9,7,8)(10,20,13,19)(11,14,12,17)(15,24,16,25)$

$(18,22,21,23),(2,7,3,6)(4,9,5,8)(10,15,13,16)(11,23,12,22)(14,21,17,18)$

$(19,24,20,25),(1,27,26)(2,24,22)(3,25,23)(4,16,21)(5,15,18)(6,19,12)(7,20,11)$

$(8,13,14)(9,10,17)])$

gap> StructureDescription(tH);

"C3 x Q8"

gap> FirstObstructionN (tG,tH).ker; \# Obs $1 \mathrm{~N}^{\sim}=\mathrm{C} 3$

$[$ [ 3$],[[2,6],[[0,2]]]]$

gap> FirstObstructionDnr (tG,tH).Dnr; \# Obs1Dnr ${ }^{\sim}=1$

$[[],[[2,6],[]]]$

gap> tGs:=AllSubgroups $(t G)$; ;

gap> Length (tGs);

138

gap> tGsHNPfalse:=Filtered(tGs, $\mathrm{x}->$ FirstObstructionDr $(\mathrm{tG}, \mathrm{x}, \mathrm{tH}) \cdot \operatorname{Dr}[1]=[])$; ;

gap> tGsHNPtrue:=Filtered ( $t G s, x->$ FirstObstructionDr $(t G, x, t H) . \operatorname{Dr}[1]=[3])$; ;

gap> List ( [tGsHNPfalse, tGsHNPtrue], Length);

$[132,6]$

gap> Collected(List (tGsHNPfalse, StructureDescription)) ;

[ [ "1", 1 ], [ "C12", 27 ], [ "C2", 9 ], [ "C3", 13 ], [ "C3 x C3", 4 ],

[ "C3 x Q8", 9 ], [ "C3 x S3", 12 ], [ "C4", 27 ], [ "C6", 9 ],

[ "Q8", 9 ], [ "S3", 12 ] ]

gap> Collected(List (tGsHNPtrue, StructureDescription));

$[$ [ $((\mathrm{C} 3 \mathrm{x} \mathrm{C} 3): \mathrm{C} 3): \mathrm{C} 2 ", 1],["((\mathrm{C} 3 \mathrm{x} \mathrm{C3}): \mathrm{C} 3): \mathrm{C} 4 ", 3]$,

[ "((C3 x C3) : C3) : Q8", 1 ], [ "(C3 x C3) : C3", 1 ] ]

gap> Collected (List (tGsHNPfalse, $\mathrm{x}->$ StructureDescription(Image (ScG.epi, $\mathrm{x}$ ))) ) ;

[ [ "1", 2 ], [ "C2", 18 ], [ "C3", 16 ], [ "C4", 54 ], [ "Q8", 18 ],

[ "S3", 24 ] ]

gap> Collected (List (tGsHNPtrue, $\mathrm{x}->$ StructureDescription (Image (ScG.epi, $\mathrm{x}$ )))) ;

[ [ "(C3 $\mathrm{x}$ C3 $): \mathrm{C} 2 ", 1],["(\mathrm{C} 3 \mathrm{x}$ C3 $): \mathrm{C} 4 ", 3],["(\mathrm{C} 3 \times \mathrm{C} 3): \mathrm{Q} 8 ", 1]$, [ "C3 $\mathrm{x}$ C3", 1 ] ]

$(3-7) G=9 T 23 \simeq\left(\left(C_{3}\right)^{2} \rtimes Q_{8}\right) \rtimes C_{3}$.

gap> Read("HNP.gap");

gap> G: $=$ TransitiveGroup $(9,23) ; \# \mathrm{G}=9 \mathrm{~T} 23=((\mathrm{C} 3 \times \mathrm{C} 3): \mathrm{Q} 8): \mathrm{C} 3$

$\mathrm{E}(9): 2 \mathrm{~A}_{-} 4$

gap> $\mathrm{H}:=$ Stabilizer $(\mathrm{G}, 1)$; \# $\mathrm{H}=\mathrm{SL}(2,3)$

Group $([(3,4,5)(6,8,7),(2,4,6)(5,9,7)])$

gap> StructureDescription $(\mathrm{H})$;

"SL $(2,3)$ "

gap> FirstObstructionN(G).ker; \# Obs1N=1 
$[[],[[3],[$ ] $]]$

gap> SchurMultPcpGroup(G); \# M(G)=C3: Schur multiplier of G

[ 3 ]

gap> ScG:=SchurCoverG $(G)$;

rec( SchurCover : $=\operatorname{Group}([(2,4,5)(3,7,6)(10,22,21)(12,19,24)(15,20,25)$

$(17,23,18),(2,5,3,6)(4,8,7,9)(10,23,17,22)(11,12,16,15)(13,18,14,21)$

$(19,25,20,24),(1,2,3)(4,10,11)(5,12,13)(6,14,15)(7,16,17)(8,18,19)(9,20,21)$

$(22,26,23)(24,27,25)])$, Tid $:=[27,212]$,

epi $:=[(2,4,5)(3,7,6)(10,22,21)(12,19,24)(15,20,25)(17,23,18)$, $(2,5,3,6)(4,8,7,9)(10,23,17,22)(11,12,16,15)(13,18,14,21)(19,25,20,24)$, $(1,2,3)(4,10,11)(5,12,13)(6,14,15)(7,16,17)(8,18,19)(9,20,21)(22,26$,

23) $(24,27,25)] \rightarrow[(3,4,5)(6,8,7),(2,8,9,3)(4,6,7,5)$, $(1,6,5)(2,7,3)(4,9,8)])$

gap> StructureDescription (TransitiveGroup $(27,212))$;

"(((C3 x C3) : C3) : Q8) : C3"

gap $>\mathrm{tG}:=\mathrm{ScG}$. SchurCover; \# $\mathrm{tG}=\mathrm{G}^{\sim}$ is a Schur cover of $\mathrm{G}$

Group $([(2,4,5)(3,7,6)(10,22,21)(12,19,24)(15,20,25)(17,23,18),(2,5,3,6)$

$(4,8,7,9)(10,23,17,22)(11,12,16,15)(13,18,14,21)(19,25,20,24),(1,2,3)$

$(4,10,11)(5,12,13)(6,14,15)(7,16,17)(8,18,19)(9,20,21)(22,26,23)(24,27,25)])$

gap> tH: $=$ PreImage (ScG.epi, H); \# tH= $\mathrm{H}^{\sim}=\mathrm{C} 3 \times \mathrm{SL}(2,3)$

Group $([(1,27,26)(2,15,17)(3,12,10)(4,20,23)(5,25,18)(6,24,21)(7,19,22)$

$(8,11,14)(9,16,13),(1,26,27)(2,13,19)(3,14,20)(4,18,15)(5,22,11)(6,23,16)$

$(7,21,12)(8,17,24)(9,10,25),(1,26,27)(2,23,25)(3,22,24)(4,18,15)(5,17,20)$

$(6,10,19)(7,21,12)(8,14,11)(9,13,16)])$

gap> StructureDescription $(\mathrm{tH})$;

"C3 x SL $(2,3) "$

gap> FirstObstructionN $(t G, t H) . k e r ; \#$ Obs $1 N^{\sim}=C 3$

$[[3],[[3,3],[[1,2]]]]$

gap> FirstObstructionDnr (tG, tH).Dnr; \# Obs1Dnr ${ }^{\sim}=1$

$[[],[[3,3],[]]]$

gap> tGs: $=A l l$ Subgroups $(t G) ;$;

gap> Length (tGs);

495

gap> tGsHNPfalse:=Filtered (tGs, $\mathrm{x}->$ FirstObstructionDr ( $t G, \mathrm{x}, \mathrm{tH}) \cdot \operatorname{Dr}[1]=[]$ ); ;

gap $>$ tGsHNPtrue :=Filtered (tGs, $\mathrm{x}->$ FirstObstructionDr (tG , $\mathrm{x}, \mathrm{tH}) \cdot \operatorname{Dr}[1]=[3]$ ); ;

gap> List ([tGsHNPfalse, tGsHNPtrue], Length);

[ 384,111$]$

gap> Collected (List(tGsHNPfalse, $\mathrm{x}$->StructureDescription(Image (ScG.epi, $\mathrm{x}$ ))));

[ [ "1", 2 ], [ "C2", 18 ], [ "C3", 88 ], [ "C4", 54 ], [ "C6", 144],

[ "Q8", 18 ], [ "S3", 24 ], [ "SL $(2,3) ", 36$ ] ]

gap> Collected(List(tGsHNPtrue, $\mathrm{x}$->StructureDescription(Image (ScG.epi, $\mathrm{x}$ ))));

[ [ "((C3 x C3) : Q8) : C3", 1$],[$ "(C3 x C3) : C2", 1$]$,

[ "(C3 x C3) : C3", 4$],[$ "(C3 $x$ C3 $): C 4 ", 3],[$ "(C3 x C3) : C6", 4 ],

[ "(C3 x C3) : Q8", 1 ], [ "C3 x C3", 49 ], [ "C3 x S3", 48 ] ]

Example 6.14 $\left(G=10 T 7 \simeq A_{5}, G=10 T 26 \simeq \mathrm{PSL}_{2}\left(\mathbb{F}_{9}\right) \simeq A_{6}\right.$ and $\left.G=10 T 32 \simeq S_{6}\right)$.

$$
\text { (4-1) } G=10 T 7 \simeq A_{5} \text {. }
$$

gap> Read ("HNP.gap");

gap> G:=TransitiveGroup $(10,7) ; \# \mathrm{G}=10 \mathrm{~T} 7=\mathrm{A} 5$

A_5 (10)

gap> H:=Stabilizer $(\mathrm{G}, 1)$; \# H=S3

Group $([(2,8)(3,6)(4,7)(5,10),(2,10)(3,9)(4,8)(5,7)])$

gap> StructureDescription $(\mathrm{H})$;

"S3"

gap> FirstObstructionN(G).ker; \# Obs $1 \mathrm{~N}=\mathrm{C} 2$

[ [ 2 ], [ [ 2 ], [ [ 11$]]]]$

gap> FirstObstructionDnr(G).Dnr; \# Obs1Dnr=1

[ [ ], [ [ 2 ], [ ] ] ]

gap> Gs:=AllSubgroups (G); ;

gap> Length(Gs);

59

gap> GsHNPfalse:=Filtered(Gs, x->FirstObstructionDr $(G, x) \cdot \operatorname{Dr}[1]=[])$; ; 


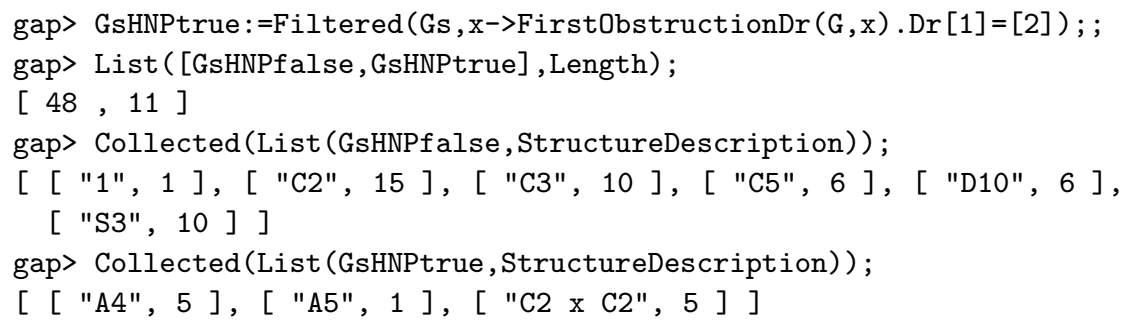


[ "C6", 120 ], [ "D10", 36 ], [ "D12", 120 ], [ "D8", 135 ], [ "S3", 160 ],

[ "S3 x S3", 20 ], [ "S4", 30 ], [ "S5", 12 ] ]

gap> Collected(List (GsHNPtrue, StructureDescription));

[ [ "A6", 1 ], [ "C2 x A4", 30 ], [ "C2 x C2", 45 ], [ "C2 x C2 x C2", 30 ],

[ "C2 x D8", 45 ], [ "C2 x S4", 30 ], [ "C4 x C2", 45 ], [ "D8", 45 ],

[ "S4", 30 ], [ "S6", 1 ] ]

gap> GsHNPtrueMin:=Filtered (GsHNPtrue, $\mathrm{x}->$ Length (Filtered (GsHNPtrue,

$>\mathrm{y}->\operatorname{IsSubgroup}(\mathrm{x}, \mathrm{y})))=1) ;$

gap> Collected(List(GsHNPtrueMin, StructureDescription));

[ [ "C2 x C2", 45 ], [ "D8", 45 ] ]

gap> GsHNPfalseC2xC2:=Filtered(GsHNPfalse, $x->\operatorname{IdSmallGroup}(\mathrm{x})=[4,2])$; ;

gap> Length(GsHNPfalseC2xC2);

120

gap> GsHNPtrueC2xC2:=Filtered (GsHNPtrue, $\mathrm{x}->\operatorname{IdSmallGroup}(\mathrm{x})=[4,2]$ ); ;

gap> Length(GsHNPtrueC2xC2); \# there exist 45 minimal true cases

45

gap> Collected(List (GsHNPfalseC2xC2, x->List (Orbits (x), Length))) ;

$[[[2,2,2,4], 3],[[2,2,4], 15],[[2,2,4,2], 6]$,

$[[2,4,2], 30],[[2,4,2,2], 9],[[4,2,2], 45]$,

$[[4,2,2,2], 12]]$

gap> Collected(List (GsHNPtrueC2xC2, x->List (Orbits (x), Length)));

$[[[2,2,2,2,2], 45]]$

gap> Collected(List(GsHNPfalseC2xC2, $\mathrm{x}->$ Collected(List ( $\mathrm{x}$,

$>\mathrm{y}^{->\operatorname{List}}(\operatorname{Orbits}(\operatorname{Group}(\mathrm{y}))$, Length)))) );

$[[[[[], 1],[[2,2,2], 2],[[2,2,2,2], 1]], 90]$,

$[[[[], 1],[[2,2,2,2], 3]], 30]]$

gap> Collected(List (GsHNPtrueC2xC2, $\mathrm{x}->$ Collected (List ( $\mathrm{x}$,

$>\mathrm{y}->\operatorname{List}(\operatorname{Orbits}(\operatorname{Group}(\mathrm{y}))$, Length)) )) );

$[[[[[], 1],[[2,2,2], 2],[[2,2,2,2], 1]], 45]]$

gap> S10:=SymmetricGroup (10);

$\operatorname{Sym}\left(\left[\begin{array}{lll}1 & \ldots & 10\end{array}\right]\right)$

gap> NS10G: $=$ Normalizer $(\mathrm{S} 10, \mathrm{G})$;

$\operatorname{Group}([(1,8,4)(2,7,5)(3,9,10),(1,5,8,10)(2,7,9,3),(1,8)(2,3)(4,6)$

$(7,9),(2,3)(5,10)(7,9),(2,10,7,5)(3,4,9,6)])$

gap> StructureDescription(NS10G);

"(A6 : C2) : C2"

gap> CS10G:=Centralizer $(\mathrm{S} 10, \mathrm{G})$;

$\operatorname{Group}(())$

gap> StructureDescription(NS10G/CS10G); \# Aut(G)=NS10G/CS10G<=S10

"(A6 : C2) : C2"

gap> Collected(List(GsHNPfalseC2xC2,

$>\mathrm{x}->$ StructureDescription (Normalizer (NS10G, x)))) ;

[ [ "C2 x D8", 90 ], [ "C2 x S4", 30 ] ]

gap> Collected (List (GsHNPtrueC2xC2,

$>\mathrm{x}->$ StructureDescription (Normalizer (NS10G, $\mathrm{x}))$ )));

[ [ "C8 : (C2 x C2)", 45$]$ ]

gap> ChG:=CharacteristicSubgroups $(G)$;

$[\operatorname{Group}(()), \operatorname{Group}([(1,8,3)(2,6,4)(5,10,7),(1,10)(2,9)(3,6)(4,7),(1,2)(3,6)$

$\left.(4,8)(5,7)]), S_{-} 6(10)=L(10): 2\right]$

gap> List (ChG, StructureDescription);

[ "1", "A6", "S6" ]

gap> GsHNPfalseD4:=Filtered(GsHNPfalse, $x->\operatorname{IdSmallGroup}(\mathrm{x})=[8,3])$; ;

gap> Length(GsHNPfalseD4);

135

gap> GsHNPtrueD4:=Filtered(GsHNPtrue, $\mathrm{x}->\operatorname{IdSmallGroup~}(\mathrm{x})=[8,3])$; ;

gap> Length(GsHNPtrueD4);

45

gap> A6:=DerivedSubgroup $(G)$;

$\operatorname{Group}([(1,8,3)(2,6,4)(5,10,7),(1,10)(2,9)(3,6)(4,7),(1,2)(3,6)(4,8)(5,7)])$

gap> Collected (List (GsHNPfalseD4, $\mathrm{x}->$ StructureDescription(Intersection (A6, $\mathrm{x})$ ))) ;

[ [ "C2 x C2", 90$],[$ "C4", 45 ] ]

gap> Collected (List (GsHNPtrueD4, $\mathrm{x}->$ StructureDescription(Intersection $(\mathrm{A} 6, \mathrm{x}))$ )) ;

[ [ "D8", 45$]$ ] 
Example 6.15 $\left(G=14 T 30 \simeq \mathrm{PSL}_{2}\left(\mathbb{F}_{13}\right)\right)$.

(5) $G=14 T 30 \simeq \operatorname{PSL}_{2}\left(\mathbb{F}_{13}\right)$.

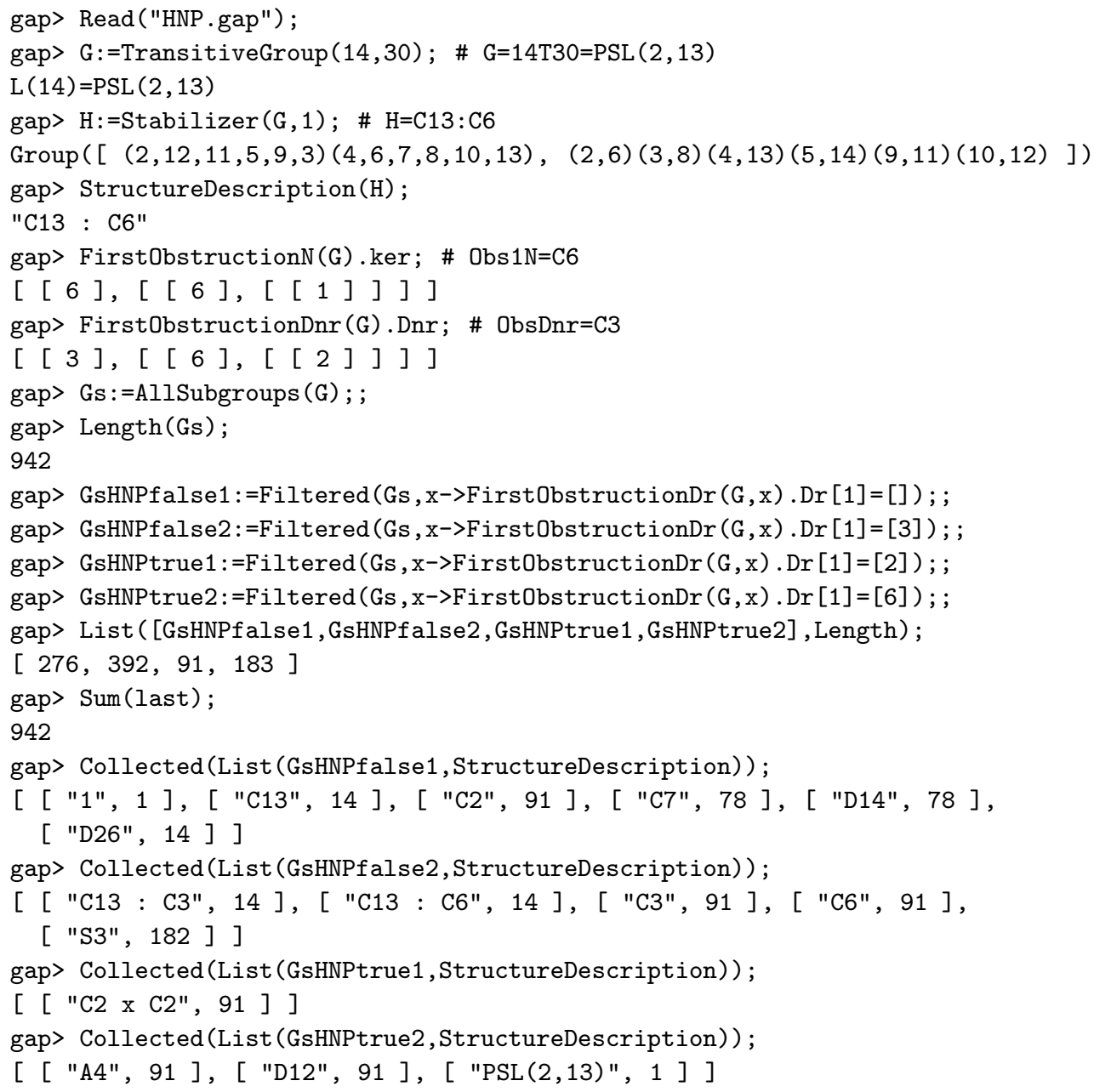

Example 6.16 $\left(G=15 T 9 \simeq\left(C_{5}\right)^{2} \rtimes C_{3}\right.$ and $\left.G=15 T 14 \simeq\left(C_{5}\right)^{2} \rtimes S_{3}\right)$.

(6-1) $G=15 T 9 \simeq\left(C_{5}\right)^{2} \rtimes C_{3}$.

gap> Read("HNP.gap");

gap> G:=TransitiveGroup $(15,9) ; \# \mathrm{G}=15 \mathrm{~T} 9=(\mathrm{C} 5 \times \mathrm{C5}): \mathrm{C} 3$

$\left[5^{\wedge} 2\right] 3$

gap> H:=Stabilizer $(\mathrm{G}, 1)$; \# H=C5

Group ( $[(2,5,8,11,14)(3,15,12,9,6)])$

gap> StructureDescription $(\mathrm{H})$;

"C5"

gap> FirstObstructionN(G).ker; \# Obs1N=C5

[ [ 5$],[[5],[[1]]]$ ]

gap> FirstObstructionDnr(G).Dnr; \# ObsDnr=1

[ [ ], [ [ 5 ], [ ] ] ]

gap> Gs:=AllSubgroups $(G) ;$;

gap> Length(Gs);

34

gap> GsHNPfalse:=Filtered(Gs, $\mathrm{x}->$ FirstObstructionDr (G,x) $\cdot \operatorname{Dr}[1]=[])$; ;

gap> GsHNPtrue:=Filtered (Gs, $\mathrm{x}->$ FirstObstructionDr $(\mathrm{G}, \mathrm{x}) \cdot \operatorname{Dr}[1]=[5])$; ;

gap> List ([GsHNPfalse, GsHNPtrue], Length);

$[32,2]$

gap> Collected(List (GsHNPfalse, StructureDescription)) ;

[ [ "1", 1 ], [ "C3", 25 ], [ "C5", 6 ] ]

gap> Collected(List (GsHNPtrue, StructureDescription));

[ [ "(C5 x C5) : C3", 1$],[$ "C5 x C5", 1$]]$

$(6-2) G=15 T 14 \simeq\left(C_{5}\right)^{2} \rtimes S_{3}$. 


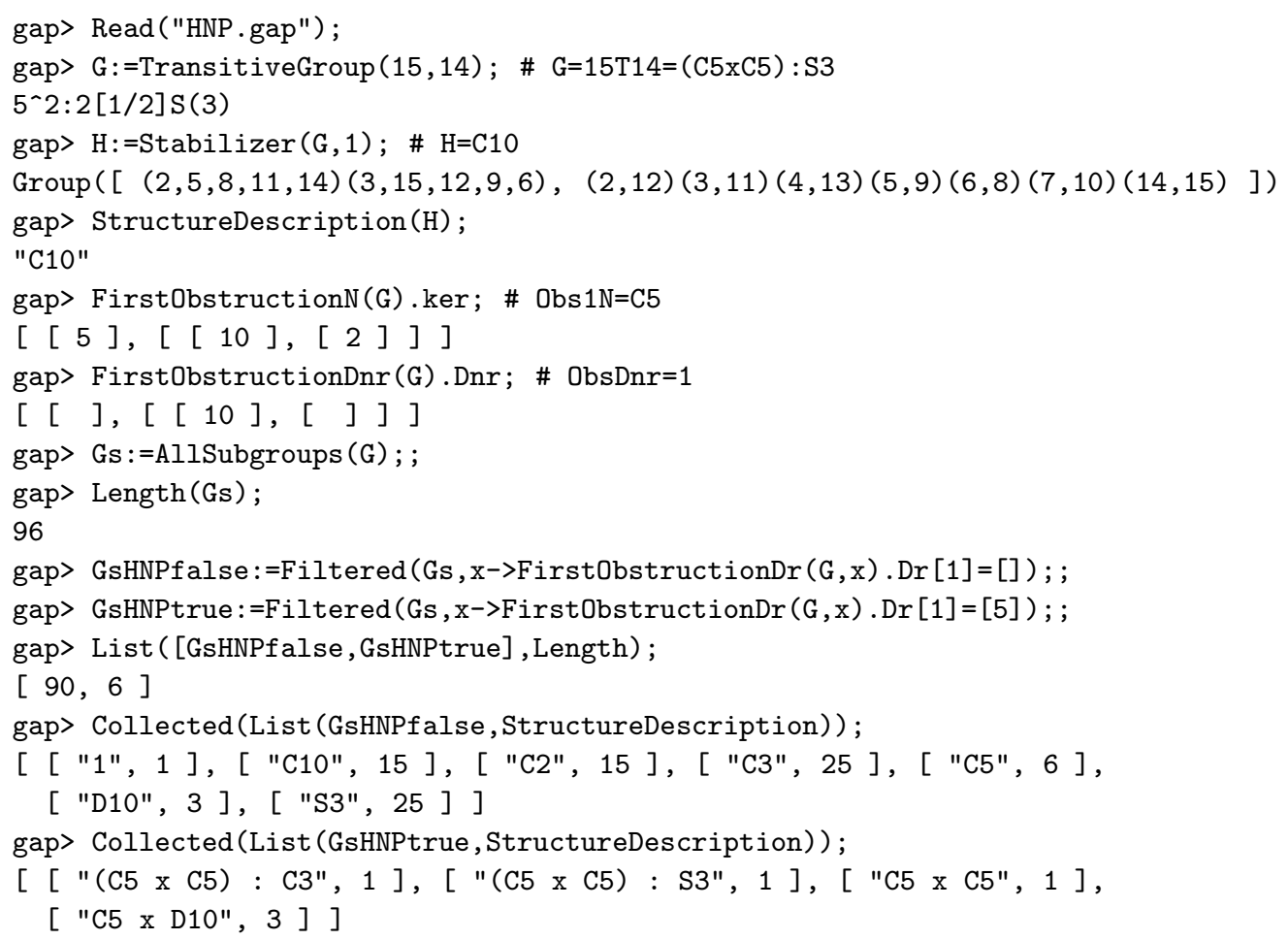

\section{Applichtion 1: $R$-eQuivalence in Algebraic $k$-TORI}

Definition 7.1. Let $k$ be a field and $T$ be an algebraic $k$-torus. A exact sequence of algebraic $k$-tori

$$
1 \rightarrow S \rightarrow Q \rightarrow T \rightarrow 1
$$

is called flabby resolution of $T$ if

$$
0 \rightarrow \widehat{T} \rightarrow \widehat{Q} \rightarrow \widehat{S} \rightarrow 0
$$

is a flabby resolution of $G$-lattice $\widehat{T}$.

Definition 7.2 (Manin [Man74, II. §14]). We say that a rational map of $k$-varieties $f: Z \rightarrow X$ covers a point $x \in X(k)$ if there exists a point $z \in Z(k)$ such that $f$ is defined at $z$ and $f(z)=x$. Two points $x, y \in X(k)$ are called $R$-equivalent if there exist a finite sequence of points $x=x_{1}, \ldots, x_{r}=y$ and rational maps $f_{i}: \mathbb{P}^{1} \rightarrow X$ $(1 \leq i \leq r-1)$ such that $f_{i}$ covers $x_{i}, x_{i+1}$.

Theorem 7.3 (Colliot-Thélène and Sansuc [CTS77, Theorem 2, page 199], see also [Vos98, Section 17.1]). Let $k$ be a field, $T$ be an algebraic $k$-torus and $1 \rightarrow S \rightarrow Q \rightarrow T \rightarrow 1$ be a flabby resolution of $T$. Then the connecting homomorphism

$$
T(k) \rightarrow H^{1}(k, S)
$$

induces an isomorphism

$$
T(k) / R \simeq H^{1}(k, S) .
$$

Theorem 7.4 (Colliot-Thélène and Sansuc [CTS77, Corollary 5, page 201], see also [Vos98, Section 17.2]). Let $k$ be a field and $T$ be an algebraic $k$-torus which splits over finite Galois extension $K$ of $k$ with $G=\operatorname{Gal}(K / k)$. Let $1 \rightarrow S \rightarrow Q \rightarrow T \rightarrow 1$ be a flabby resolution of $T$. Then

(i) If $k=\mathbb{F}_{q}$ or a field of cohomological dimension $\operatorname{cd}(k) \leq 1$, then

$$
T(k) / R=0 ;
$$

(ii) If $k$ is a local field, then

$$
T(k) / R \simeq H^{1}(G, \widehat{S})^{\vee} ;
$$

(iii) If $k$ is a global field, then there exists an exact sequence

$$
0 \rightarrow \amalg^{2}(G, \widehat{S})^{\vee} \rightarrow T(k) / R \rightarrow \mathrm{บ}^{1}(G, \widehat{S})^{\vee} \rightarrow 0
$$


where

$$
\begin{aligned}
& \amalg^{2}(G, \widehat{S})=\operatorname{Ker}\left\{H^{2}(G, \widehat{S}) \stackrel{\text { res }}{\longrightarrow} \bigoplus_{v \in V_{k}} H^{2}\left(G_{v}, \widehat{S}\right)\right\}, \\
& \mathrm{\Psi}^{1}(G, \widehat{S})=\operatorname{Coker}\left\{H^{1}(G, \widehat{S}) \stackrel{\text { res }}{\longrightarrow} \bigoplus_{v \in V_{k}} H^{1}\left(G_{v}, \widehat{S}\right)\right\} .
\end{aligned}
$$

When $T=R_{K / k}^{(1)}\left(\mathbb{G}_{m}\right)$ and $K / k$ is a finite Galois extension, we have $\widehat{T}=J_{G}$ and $H^{1}(k, \widehat{S}) \simeq H^{3}(G, \mathbb{Z})$. Hence Theorem 7.4 can be stated as follows:

Theorem 7.5 (Colliot-Thélène and Sansuc [CTS77, Corollary 1, page 207], see also [Vos98, Section 17.2]). Let $k$ be a field and $K / k$ be a finite Galois extension with Galois group $G=\operatorname{Gal}(K / k)$. Let $T=R_{K / k}^{(1)}\left(\mathbb{G}_{m}\right)$ be the norm one torus defined by $K / k$. Then

(i) If $k=\mathbb{F}_{q}$ or a field of cohomological dimension $\operatorname{cd}(k) \leq 1$, then

$$
T(k) / R=0 ;
$$

(ii) If $k$ is a local field, then

$$
T(k) / R \simeq H^{3}(G, \mathbb{Z})^{\vee}
$$

(iii) If $k$ is a global field, then there exists an exact sequence

$$
0 \rightarrow \amalg^{4}(G, \mathbb{Z})^{\vee} \rightarrow T(k) / R \rightarrow \mathrm{\Psi}^{3}(G, \mathbb{Z})^{\vee} \rightarrow 0 .
$$

When $k$ is a local field, Voskresenskii's theorem ([Vos67]), Kunyavskii's theorem (Theorem 1.4), Theorem 4.1 and Theorem 4.2 enable us to get $T(k) / R$ for algebraic $k$-tori $T$ of dimension $\leq 5$. We also refer to Merkurjev Mer08 for algebraic $k$-tori $T$ of dimension 3.

Theorem 7.6. Let $k$ be a local field and $T$ be an algebraic $k$-torus of dimension $n \leq 5$. Then

$$
T(k) / R \leq \begin{cases}0 & (n=1,2), \\ \mathbb{Z} / 2 \mathbb{Z} & (n=3), \\ (\mathbb{Z} / 2 \mathbb{Z})^{\oplus 2} & (n=4,5)\end{cases}
$$

and $T(k) / R \simeq H^{1}\left(G,\left[\widehat{T}^{f l}{ }^{f l}\right)\right.$ is given as in Theorem 1.4 $(n=3)$, Theorem 4.1 $(n=4)$ and Theorem 4.2 $(n=5)$.

Also, Theorem 1.15 enables us to obtain $T(k) / R \simeq H^{1}(k, \operatorname{Pic} \bar{X}) \simeq H^{1}\left(G,\left[J_{G / H}\right]^{f l}\right)$ for norm one tori $T=$ $R_{K / k}^{(1)}\left(\mathbb{G}_{m}\right)$ where $k$ is a local field and $[K: k]=n \leq 15$ and $n \neq 12$.

Theorem 7.7. Let $2 \leq n \leq 15$ be an integer with $n \neq 12$. Let $k$ be a local field, $K / k$ be a separable field extension of degree $n$ and $L / k$ be the Galois closure of $K / k$. Assume that $G=\operatorname{Gal}(L / k)=n T m$ is a transitive subgroup of $S_{n}$ and $H=\operatorname{Gal}(L / K)$ with $[G: H]=n$. Let $T=R_{K / k}^{(1)}\left(\mathbb{G}_{m}\right)$ be the norm one torus of $K / k$ of dimension $n-1$. Then

$$
T(k) / R \simeq H^{1}\left(G,\left[J_{G / H}\right]^{f l}\right) \leq \begin{cases}0 & (n=2,3,5,7,11,13) \\ \mathbb{Z} / 2 \mathbb{Z} & (n=4,6,10,14), \\ (\mathbb{Z} / 2 \mathbb{Z})^{\oplus 3} & (n=8), \\ \mathbb{Z} / 3 \mathbb{Z} & (n=9), \\ \mathbb{Z} / 5 \mathbb{Z} & (n=15)\end{cases}
$$

and $T(k) / R \neq 0$ if and only if $G$ is given as in Table 1.

\section{Application 2: Tamagawa number $\tau(T)$}

By Theorem 1.18, we obtain the Tamagawa number $\tau(T)$ of algebraic $k$-tori $T$ (see Ono Ono63, Ono65] and Voskresenskii Vos98, Chapter 5]).

Theorem 8.1 (Ono [Ono63, Main theorem, page 68], see also [Vos98, Theorem 2, page 146]). Let $k$ be a global field, $T$ be an algebraic $k$-torus and $\tau(T)$ be the Tamagawa number of $T$. Then

$$
\tau(T)=\frac{\left|H^{1}(k, \widehat{T})\right|}{|\amalg(T)|} .
$$

In particular, if $T$ is retract $k$-rational, then $\tau(T)=\left|H^{1}(k, \widehat{T})\right|$.

For the last assertion, see Theorem [3.6. As a consequence of Theorem 1.1, Theorem 4.1 and Theorem 4.2 (Theorem 1.5] and Theorem [1.6), we have: 
Theorem 8.2. Let $k$ be a global field and $T$ be an algebraic $k$-torus of dimension 4 (resp. 5). Among 710 (reps. 6079) cases of algebraic $k$-tori $T$, if $T$ is one of the 688 (resp. 5805) cases with $H^{1}(k, \operatorname{Pic} \bar{X})=0$, then $\tau(T)=\left|H^{1}(k, \widehat{T})\right|$.

When $T=R_{K / k}^{(1)}\left(\mathbb{G}_{m}\right)$ and $K / k$ is a finite Galois extension, i.e. $\widehat{T}=J_{G}$, it follows from Endo and Miyata EM75, Theorem 1.5] that if all the Sylow subgroups of $G=\operatorname{Gal}(K / k)$ are cyclic, then $|\amalg(T)|=1$ and hence $\tau(T)=\left|H^{1}\left(G, J_{G}\right)\right|=\left|H^{2}(G, \mathbb{Z})\right|=\left|H^{1}(G, \mathbb{Q} / \mathbb{Z})\right|=\left|G^{a b}\right|$. For norm one tori $T=R_{K / k}^{(1)}\left(\mathbb{G}_{m}\right)$ with $[K: k]=$ $n \leq 15$ and $n \neq 12$, Kunyavskii's theorem (Theorem 1.13), Drakokhrust and Platonov's theorem (Theorem 1.14) and Theorem 1.18 enable us to get the Tamagawa number $\tau(T)$ :

Theorem 8.3. Let $2 \leq n \leq 15$ be an integer with $n \neq 12$. Let $k$ be a number field, $K / k$ be a field extension of degree $n$ and $L / k$ be the Galois closure of $K / k$. Assume that $G=\operatorname{Gal}(L / k)=n$ Tm is a transitive subgroup of $S_{n}$ and $H=\operatorname{Gal}(L / K)$ with $[G: H]=n$. Let $T=R_{K / k}^{(1)}\left(\mathbb{G}_{m}\right)$ be the norm one torus of $K / k$ of dimension $n-1$. Then $\tau(T)=\left|H^{1}\left(G, J_{G / H}\right)\right|$ except for the cases in Table 1 . For the cases in Table 1 , we have $\tau(T)=$ $\left|H^{1}\left(G, J_{G / H}\right)\right| /|\amalg(T)|$ where $H^{1}\left(G, J_{G / H}\right)$ is given as in Section 9 and $\amalg(T)$ is given as in Theorem 1.18 .

We give GAP computations of $H^{1}\left(G, J_{G / H}\right)$ for $G=\operatorname{Gal}(L / k)=n T m(n \leq 15)$ in Section 9 as the appendix of this paper.

9. Appendix: Computation of $H^{1}\left(G, J_{G / H}\right)$ For $G=\operatorname{Gal}(L / k)=n T m(n \leq 15)$

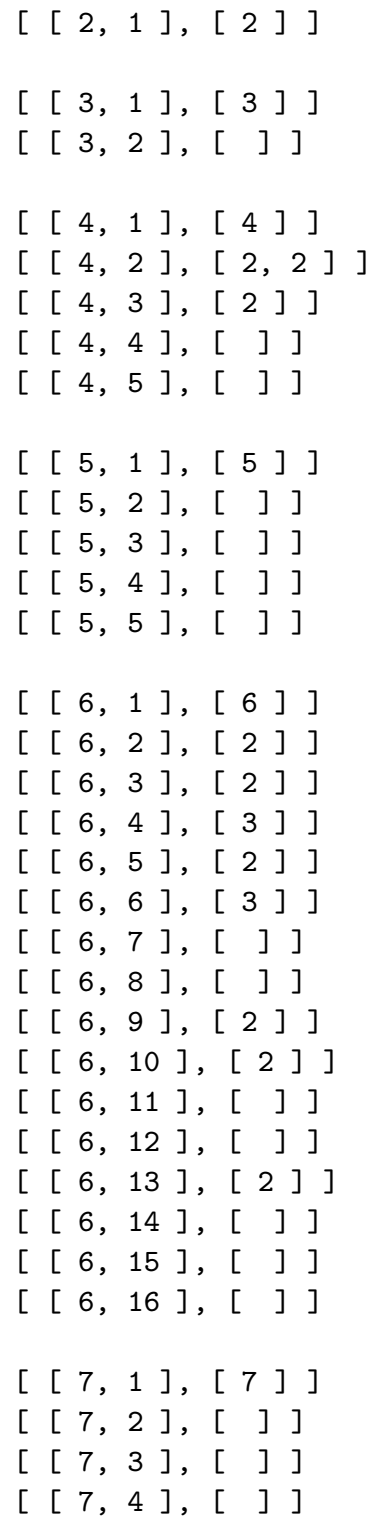


$[[7,5],[]]$

$[[7,6],[]]$

$[[7,7],[]]$

$[[8,1],[8]]$

$[[8,2],[2,4]]$

$[[8,3],[2,2,2]]$

$[[8,4],[2,2]]$

$[[8,5],[2,2]]$

$[[8,6],[2]]$

$[[8,7],[4]]$

$[[8,8],[2]]$

$[[8,9],[2,2]]$

$[[8,10],[4]]$

$[[8,11],[2,2]]$

$[[8,12],[]]$

$[[8,13],[2]]$

$[[8,14],[2]]$

$[[8,15],[2]]$

$[[8,16],[4]]$

$[[8,17],[2]]$

$[[8,18],[2]]$

$[[8,19],[2]]$

$[[8,20],[4]]$

$[[8,21],[2,2]]$

$[[8,22],[2,2]]$

$[[8,23],[]]$

$[[8,24],[2]]$

$[[8,25],[]]$

$[[8,26],[2]]$

$[[8,27],[4]]$

$[[8,28],[2]]$

$[[8,29],[2]]$

$[[8,30],[2]]$

$[[8,31],[2,2]]$

$[[8,32],[]]$

$[[8,33],[2]]$

$[[8,34],[2]]$

$[[8,35],[2]]$

$[[8,36],[]]$

$[[8,37],[]]$

$[[8,38],[]]$

$[[8,39],[]]$

$[[8,40],[]]$

$[[8,41],[2]]$

$[[8,42],[2]]$

$[[8,43],[]]$

$[[8,44],[]]$

$[[8,45],[2]]$

$[[8,46],[2]]$

$[[8,47],[2]]$

$[[8,48],[]]$

$[[8,49],[]]$

$[[8,50],[]]$

[ $[9,1],[9]]$

$[[9,2],[3,3]]$

$[[9,3],[]]$

$[[9,4],[3]]$

$[[9,5],[]]$

$[[9,6],[3]]$

$[[9,7],[3]]$

$[[9,8],[]]$ 
$[[9,9],[]]$

$[[9,10],[]]$

$[[9,11],[]]$

$[[9,12],[]]$

$[[9,13],[3]]$

$[[9,14],[]]$

$[[9,15],[]]$

$[[9,16],[]]$

$[[9,17],[3]]$

$[[9,18],[]]$

$[[9,19],[]]$

$[[9,20],[]]$

$[[9,21],[]]$

$[[9,22],[3]]$

$[[9,23],[]]$

$[[9,24],[]]$

$[[9,25],[3]]$

$[[9,26],[]]$

$[[9,27],[]]$

$[[9,28],[3]]$

$[[9,29],[]]$

$[[9,30],[]]$

$[[9,31],[]]$

$[[9,32],[]]$

$[[9,33],[]]$

$[[9,34],[]]$

$[[10,1],[10]]$

[ [ 10,2 ], [ 2$]]$

[ $[10,3],[2]]$

$[[10,4],[2]]$

$[[10,5],[2]]$

$[[10,6],[2]]$

$[[10,7],[]]$

$[[10,8],[5]]$

$[[10,9],[2]]$

[ [ 10,10], [ 2 ] ]

$[[10,11],[2]]$

$[[10,12],[2]]$

$[[10,13],[]]$

[ [ 10,14], [ 5$]$ ]

[ [ 10,15], [ ] ]

$[[10,16],[]]$

[ $[10,17],[2]]$

[ [ 10,18], [ 2$]]$

$[[10,19],[2]]$

[ [ 10,20], [ 2$]]$

[ [ 10,21], [ 2 ] ]

[ [ 10,22], [ 2$]]$

[ $[10,23],[]]$

$[[10,24],[]]$

$[[10,25],[]]$

$[[10,26],[]]$

[ [ 10,27 ], [ 2$]]$

[ [ 10, 28 ], [ 2 ] ]

[ [ 10,29], [ ] ]

$[[10,30],[]]$

$[[10,31],[]]$

[ [ 10,32], [ ] ]

[ $[10,33],[2]]$

$[[10,34],[]]$

[ [ 10,35], [ ] ]

$[[10,36],[]]$ 
[ [ 10,37$],[$ ] ]

$[[10,38],[]]$

[ [ 10,39], [ ] ]

[ [ 10,40], [ 2$]]$

[ [ 10,41], [ 2$]]$

[ [ 10,42], [ 2 ] ]

$[[10,43],[2]]$

[ [ 10,44], [ ] ]

$[[10,45],[]]$

[ [ 11,1$],[11]]$

$[[11,2],[]]$

$[[11,3],[]]$

$[[11,4],[]]$

$[[11,5],[]]$

$[[11,6],[]]$

$[[11,7],[]]$

$[[11,8],[]]$

[ [ 12,1$],[12]]$

$[[12,2],[2,6]]$

[ $[12,3],[2,2]]$

[ [ 12,4$],[3]]$

$[[12,5],[4]]$

$[[12,6],[3]]$

$[[12,7],[6]]$

$[[12,8],[]]$

[ [ 12,9$],[2]]$

$[[12,10],[2,2]]$

[ [ 12,11$],[4]]$

[ [ 12,12$],[2]]$

[ $[12,13],[2]]$

$[[12,14],[6]]$

[ [ 12,15$],[2]]$

$[[12,16],[2,2]]$

[ [ 12,17$],[4]]$

[ $[12,18],[2,2]]$

[ [ 12,19$],[4]]$

$[[12,20],[3]]$

[ [ 12, 21 ], [ 2$]]$

[ [ 12, 22 ], [ ] ]

[ [ 12, 23], [ 2 ] ]

[ [ 12, 24 ], [ 2$]]$

$[[12,25],[6]]$

[ [ 12, 26 ], [ 3$]]$

[ [ 12, 27 ], [ ] ]

[ [ 12, 28 ], [ 2$]]$

[ [ 12, 29], [6] ]

[ [ 12,30], [ 2$]]$

$[[12,31],[3]]$

[ $[12,32],[3]]$

[ [ 12, 33 ], [ ] ]

$[[12,34],[2,2]]$

[ [ 12,35], [ 2 ] ]

[ [ 12,36], [ 2 ] ]

[ $[12,37],[2,2]]$

$[[12,38],[2]]$

$[[12,39],[4]]$

$[[12,40],[2,2]]$

[ [ 12,41$],[4]]$

[ [ 12,42], [ 2$]]$

[ [ 12,43], [ ] ]

[ [ 12,44$],[]]$ 
$[[12,45],[3]]$

$[[12,46],[4]]$

$[[12,47],[2,2]]$

[ [ 12,48$],[2]]$

[ [ 12,49$],[\mathrm{]}]$

$[[12,50],[2]]$

$[[12,51],[6]]$

$[[12,52],[2]]$

$[[12,53],[2]]$

[ [ 12,54], [ 2$]]$

$[[12,55],[3]]$

$[[12,56],[3]]$

$[[12,57],[3]]$

$[[12,58],[6]]$

$[[12,59],[3]]$

$[[12,60],[3]]$

[ [ 12,61], [ 3$]$ ]

[ [ 12,62$],[]]$

$[[12,63],[]]$

[ [ 12,64$],[]]$

[ [ 12,65$],[]]$

$[[12,66],[]]$

$[[12,67],[]]$

[ [ 12,68 ], [ ] ]

[ [ 12,69], [ 2$]]$

$[[12,70],[2,2]]$

$[[12,71],[2,2]]$

[ $[12,72],[4]]$

[ [ 12,73], [ 4$]]$

$[[12,74],[2]]$

[ $[12,75],[2]]$

$[[12,76],[]]$

$[[12,77],[2,2]]$

[ [ 12,78], [ 2 ] ]

[ [ 12,79], [ 4 ] ]

[ [ 12,80], [ 2 ] ]

[ [ 12,81], [ 2 ] ]

[ [ 12,82], [ 2 ] ]

[ [ 12,83$],[\mathrm{]}]$

[ [ 12,84], [ 2 ] ]

[ [ 12,85$],[3]]$

[ [ 12,86], [ 2$]]$

[ $[12,87],[6]]$

[ $[12,88],[3]]$

[ [ 12,89$],[3]]$

[ [ 12,90$],[3]]$

[ [ 12,91$],[3]]$

[ [ 12,92$],[3]]$

[ [ 12,93 ], [ 3 ] ]

[ [ 12,94], [ 3$]$ ]

[ [ 12,95$],[$ ] ]

[ [ 12,96$],[]]$

[ [ 12,97$],[]]$

[ [ 12,98 ], [ ] ]

[ [ 12,99], [ 3$]$ ]

[ [ 12,100$],[]]$

[ [ 12,101$],[]]$

$[[12,102],[]]$

[ $[12,103],[]]$

[ [ 12, 104$],[3]]$

$[[12,105],[6]]$

[ [ 12,106$],[2]]$

[ [ 12, 107$],[2]]$ 
[ [ 12,108$],[2]]$

[ [ 12,109$],[2]]$

[ [ 12,110$],[]]$

[ [ 12, 111], [ ] ]

[ [ 12,112$],[]]$

[ [ 12,113$],[]]$

[ $[12,114],[]]$

[ [ 12,115$],[$ ] ]

$[[12,116],[2]]$

$[[12,117],[2,2]]$

[ [ 12,118$],[2]]$

[ [ 12,119$],[4]]$

$[[12,120],[2]]$

[ [ 12,121$],[2]]$

[ [ 12,122], [ ] ]

$[[12,123],[2]]$

[ [ 12,124], [ ] ]

$[[12,125],[2]]$

[ $[12,126],[2]]$

[ [ 12,127$],[$ ] ]

[ [ 12,128 ], [ ] ]

[ [ 12,129$],[3]]$

[ $[12,130],[2,2]]$

[ [ 12,131$],[4]]$

[ [ 12,132], [ ] ]

[ $[12,133],[\mathrm{]}]$

$[[12,134],[6]]$

[ [ 12,135$],[2]]$

$[[12,136],[2]]$

[ [ 12,137 ], [ ] ]

[ [ 12,138], [ ] ]

[ $[12,139],[]]$

[ [ 12,140], [ ] ]

[ $[12,141],[3]]$

$[[12,142],[3]]$

[ [ 12,143], [ 3$]]$

[ [ 12,144$],[3]]$

$[[12,145],[2]]$

[ [ 12,146], [ ] ]

[ [ 12,147 ], [ ] ]

[ [ 12,148], [ ] ]

[ [ 12,149], [ ] ]

[ [ 12,150], [ ] ]

[ [ 12, 151 ], [ ] ]

$[[12,152],[]]$

[ [ 12,153], [ ] ]

[ [ 12,154$],[2]]$

$[[12,155],[2]]$

[ [ 12,156], [ 2$]]$

[ [ 12,157 ], [ ] ]

[ [ 12,158$],[2]]$

[ [ 12,159$],[2]]$

$[[12,160],[2]]$

[ [ 12,161$],[2]]$

$[[12,162],[2]]$

[ [ 12, 163$],[2]]$

[ $[12,164],[3]]$

$[[12,165],[]]$

[ $[12,166],[3]]$

[ [ 12,167$],[2]]$

[ [ 12, 168 ], [2, 2] ]

[ [ 12,169$],[2]]$

[ [ 12,170$],[4]]$ 
[ [ 12,171$],[2,2]]$

$[[12,172],[2,2]]$

$[[12,173],[4]]$

[ $[12,174],[2,2]]$

[ [ 12,175], [ ] ]

$[[12,176],[]]$

[ [ 12,177$],[\mathrm{]}]$

[ [ 12,178$],[]]$

$[[12,179],[]]$

$[[12,180],[2]]$

$[[12,181],[2]]$

$[[12,182],[2]]$

$[[12,183],[2]]$

[ [ 12,184$],[]]$

[ [ 12,185$],[$ ] ]

[ [ 12,186 ], [ ] ]

[ [ 12,187$],[3]]$

[ [ 12,188$],[3]]$

[ [ 12,189$],[3]]$

[ [ 12, 190], [ ] ]

[ [ 12, 191 ], [ ] ]

[ [ 12, 192 ], [ ] ]

[ [ 12,193$],[2]]$

[ [ 12, 194 ], [ ] ]

[ [ 12,195$],[2]]$

$[[12,196],[2]]$

[ [ 12,197$],[2]]$

[ [ 12,198$],[2]]$

[ [ 12, 199$],[2]]$

[ [ 12, 200 ], [ 2 ] ]

[ [ 12, 201$],[2]]$

[ [ 12, 202], [2] ]

[ [ 12, 203], [ 2$]]$

[ [ 12, 204 ], [ ] ]

[ [ 12, 205 ], [ 3$]]$

[ [ 12, 206 ], [ ] ]

[ [ 12, 207 ], [ ] ]

[ [ 12, 208 ], [ 2$]]$

[ [ 12, 209], [2] ]

[ [ 12, 210], [2, 2] ]

[ [ 12,211$],[4]]$

[ [ 12, 212 ], [ 2$]]$

[ [ 12, 213], [ ] ]

[ [ 12, 214], [2, 2] ]

[ [ 12,215$],[4]]$

[ [ 12,216$],[2]]$

[ [ 12,217$],[2]]$

[ [ 12, 218 ], [ ] ]

[ [ 12, 219 ], [ 2 ] ]

[ [ 12, 220 ], [2] ]

[ [ 12, 221 ], [ ] ]

[ [ 12,222$],[3]]$

[ [ 12, 223 ], [ ] ]

[ [ 12, 224 ], [ ] ]

[ [ 12, 225 ], [ ] ]

[ [ 12,226$],[$ ] ]

[ [ 12, 227 ], [ ] ]

[ [ 12,228$],[3]]$

[ [ 12,229$],[3]]$

[ [ 12, 230 ], [ ] ]

[ [ 12, 231 ], [ ] ]

[ [ 12, 232 ], [ ] ]

[ $[12,233],[$ ] $]$ 
[ [ 12, 234 ], [ ] ]

$[[12,235],[2]]$

$[[12,236],[2]]$

[ [ 12,237$],[2]]$

[ [ 12,238$],[2]]$

[ [ 12, 239], [ ] ]

$[[12,240],[2]]$

[ [ 12,241$],[2]]$

[ [ 12, 242 ], [ 2, 2] ]

[ [ 12,243$],[2]]$

[ [ 12, 244 ], [ 4 ] ]

[ [ 12, 245], [ 4$]]$

$[[12,246],[2,2]]$

[ [ 12,247$],[2]]$

[ [ 12, 248], [ 2 ] ]

[ [ 12, 249], [ 2 ] ]

[ [ 12, 250], [ ] ]

[ [ 12, 251], [ ] ]

[ [ 12, 252 ], [ ] ]

[ [ 12,253$],[3]]$

[ [ 12, 254 ], [ ] ]

[ [ 12, 255 ], [ ] ]

[ [ 12, 256 ], [ ] ]

[ [ 12, 257 ], [ ] ]

[ [ 12, 258 ], [ ] ]

[ [ 12, 259 ], [ ] ]

[ [ 12,260$],[2]]$

[ [ 12, 261 ], [ 2,2 ] ]

[ [ 12, 262 ], [ 2$]]$

[ [ 12,263 ], [ 2 ] ]

[ [ 12,264$],[4]]$

[ [ 12, 265 ], [ 3 ] ]

[ [ 12,266$],[2]]$

[ [ 12, 267 ], [ 2$]]$

[ [ 12, 268 ], [ ] ]

[ [ 12, 269 ], [ 2 ] ]

[ [ 12, 270], [ ] ]

[ [ 12, 271 ], [ ] ]

[ [ 12, 272 ], [ ] ]

$[[12,273],[3]]$

[ [ 12,274$],[2]]$

[ [ 12, 275 ], [ ] ]

[ [ 12, 276 ], [ ] ]

[ [ 12, 277 ], [ ] ]

[ [ 12, 278 ], [2] ]

[ [ 12, 279 ], [ 2 ] ]

[ [ 12, 280 ], [ ] ]

[ [ 12, 281 ], [ ] ]

[ [ 12, 282 ], [ ] ]

[ [ 12, 283 ], [ ] ]

[ [ 12,284$],[3]]$

[ [ 12, 285 ], [ ] ]

[ [ 12, 286 ], [ ] ]

[ [ 12, 287 ], [ ] ]

[ [ 12, 288 ], [ 2 ] ]

[ [ 12, 289 ], [ ] ]

[ [ 12, 290 ], [ ] ]

[ [ 12, 291 ], [ ] ]

[ [ 12,292$],[3]]$

[ [ 12, 293 ], [ ] ]

[ [ 12, 294 ], [ ] ]

[ [ 12, 295 ], [ ] ]

[ [ 12, 296 ], [ 2 ] ] 
[ [ 12,297$],[2]]$

[ [ 12,298$],[2]]$

$[[12,299],[2]]$

$[[12,300],[]]$

$[[12,301],[]]$

$[[13,1],[13]]$

$[[13,2],[]]$

$[[13,3],[]]$

$[[13,4],[]]$

$[[13,5],[]]$

$[[13,6],[]]$

$[[13,7],[]]$

$[[13,8],[]]$

$[[13,9],[]]$

$[[14,1],[14]]$

[ [ 14,2 ], [ 2$]]$

$[[14,3],[2]]$

$[[14,4],[2]]$

$[[14,5],[2]]$

$[[14,6],[7]]$

[ [ 14,7$],[2]]$

[ [ 14, 8 ], [ 2$]]$

$[[14,9],[7]]$

$[[14,10],[]]$

$[[14,11],[]]$

[ [ 14,12$],[2]]$

[ [ 14,13], [ 2 ] ]

[ [ 14,14], [ 2$]]$

[ [ 14,15], [ 2$]]$

[ [ 14, 16], [ 2] ]

[ $[14,17],[]]$

[ $[14,18],[]]$

[ [ 14, 19], [ 2$]]$

[ [ 14, 20], [ 2$]]$

[ [ 14,21], [ 7 ] ]

[ [ 14, 22 ], [ 2$]]$

$[[14,23],[2]]$

$[[14,24],[2]]$

[ [ 14,25], [ 2 ] ]

[ [ 14, 26 ], [ 2$]]$

[ [ 14,27 ], [ ] ]

[ [ 14, 28 ], [ ] ]

$[[14,29],[7]]$

$[[14,30],[]]$

[ [ 14, 31 ], [ 2 ] ]

[ [ 14,32 ], [ 2$]]$

[ [ 14,33 ], [ ] ]

[ $[14,34],[]]$

$[[14,35],[]]$

$[[14,36],[2]]$

[ [ 14,37 ], [ 2 ] ]

[ [ 14,38 ], [ ] ]

[ [ 14,39], [ ] ]

[ [ 14,40], [ ] ]

$[[14,41],[]]$

$[[14,42],[]]$

[ [ 14,43], [ ] ]

[ [ 14,44], [ ] ]

[ [ 14,45], [ 2$]]$

[ [ 14,46], [ 2 ] ]

[ [ 14,47], [2] ] 
[ [ 14,48$],[\mathrm{]}]$

[ [ 14,49$],[2]]$

[ [ 14,50$],[]]$

[ $[14,51],[]]$

$[[14,52],[2]]$

[ [ 14,53], [ ] ]

[ [ 14,54 ], [ ] ]

[ [ 14,55$],[]]$

[ [ 14,56], [ ] ]

[ [ 14,57 ], [ ] ]

[ [ 14,58], [ 2$]]$

[ [ 14,59], [ 2$]]$

$[[14,60],[2]]$

$[[14,61],[2]]$

$[[14,62],[]]$

[ $[14,63],[]]$

[ [ 15, 1], [ 15$]]$

$[[15,2],[]]$

$[[15,3],[3]]$

$[[15,4],[5]]$

$[[15,5],[]]$

$[[15,6],[]]$

$[[15,7],[]]$

$[[15,8],[3]]$

$[[15,9],[3]]$

[ $[15,10],[]]$

$[[15,11],[]]$

[ $[15,12],[3]]$

[ [ 15, 13 ], [ ] ]

$[[15,14],[]]$

$[[15,15],[]]$

$[[15,16],[3]]$

[ $[15,17],[]]$

$[[15,18],[]]$

[ [ 15, 19], [ 3 ] ]

$[[15,20],[]]$

[ [ 15, 21 ], [ ] ]

$[[15,22],[]]$

$[[15,23],[]]$

[ [ 15, 24], [ 3 ] ]

$[[15,25],[3]]$

[ [ 15, 26 ], [ 5 ] ]

$[[15,27],[]]$

$[[15,28],[]]$

$[[15,29],[]]$

[ [ 15,30$],[3]]$

[ [ 15, 31 ], [ ] ]

$[[15,32],[]]$

[ $[15,33],[5]]$

$[[15,34],[]]$

$[[15,35],[]]$

$[[15,36],[5]]$

[ [ 15,37$],[]]$

[ [ 15, 38 ], [ 3 ] ]

[ $[15,39],[3]]$

$[[15,40],[]]$

$[[15,41],[]]$

[ [ 15,42], [ ] ]

[ [ 15,43], [ ] ]

[ [ 15,44], [ 5$]$ ]

[ [ 15,45], [ ] ]

[ $[15,46],[]]$ 
[ [ 15,47 ], [ ] ]

[ [ 15,48], [ ] ]

[ [ 15,49], [ ] ]

[ $[15,50],[3]]$

[ [ 15, 51 ], [ ] ]

[ [ 15, 52 ], [ ] ]

$[[15,53],[]]$

[ [ 15,54 ], [ ] ]

$[[15,55],[]]$

$[[15,56],[]]$

[ [ 15,57 ], [ 3 ] ]

$[[15,58],[]]$

[ [ 15, 59 ], [ 3 ] ]

$[[15,60],[]]$

$[[15,61],[]]$

[ [ 15,62 ], [ ] ]

[ [ 15,63], [ ] ]

[ $[15,64],[]]$

$[[15,65],[]]$

$[[15,66],[]]$

[ [ 15,67 ], [ 3$]]$

$[[15,68],[]]$

$[[15,69],[]]$

$[[15,70],[]]$

$[[15,71],[5]]$

[ $[15,72],[]]$

$[[15,73],[]]$

[ [ 15,74], [ ] ]

$[[15,75],[3]]$

$[[15,76],[]]$

[ [ 15,77 ], [ ] ]

$[[15,78],[]]$

[ [ 15,79], [ ] ]

$[[15,80],[]]$

[ [ 15,81$],[5]]$

[ [ 15,82], [ ] ]

$[[15,83],[]]$

[ $[15,84],[]]$

$[[15,85],[]]$

$[[15,86],[]]$

[ [ 15,87 ], [ ] ]

[ [ 15,88 ], [ ] ]

[ [ 15,89 ], [ ] ]

$[[15,90],[]]$

[ [ 15,91 ], [ ] ]

[ [ 15,92 ], [ 3$]]$

[ [ 15,93$],[]]$

$[[15,94],[]]$

[ $[15,95],[3]]$

$[[15,96],[]]$

[ [ 15,97$],[]]$

[ [ 15,98], [ 3$]]$

[ [ 15,99 ], [ ] ]

[ [ 15, 100], [ ] ]

$[[15,101],[3]]$

[ [ 15, 102 ], [ ] ]

[ $[15,103],[]]$

$[[15,104],[]]$

\section{GAP ALGORITHMS}

We give GAP algorithms for computing the total obstruction $\operatorname{Obs}(K / k)$ and the first obstruction $\operatorname{Obs}_{1}(L / K / k)$ as in Section 6. The functions which are provided in this section are available from

https://www.math.kyoto-u.ac.jp/ yamasaki/Algorithm/Norm1ToriHNP/. 


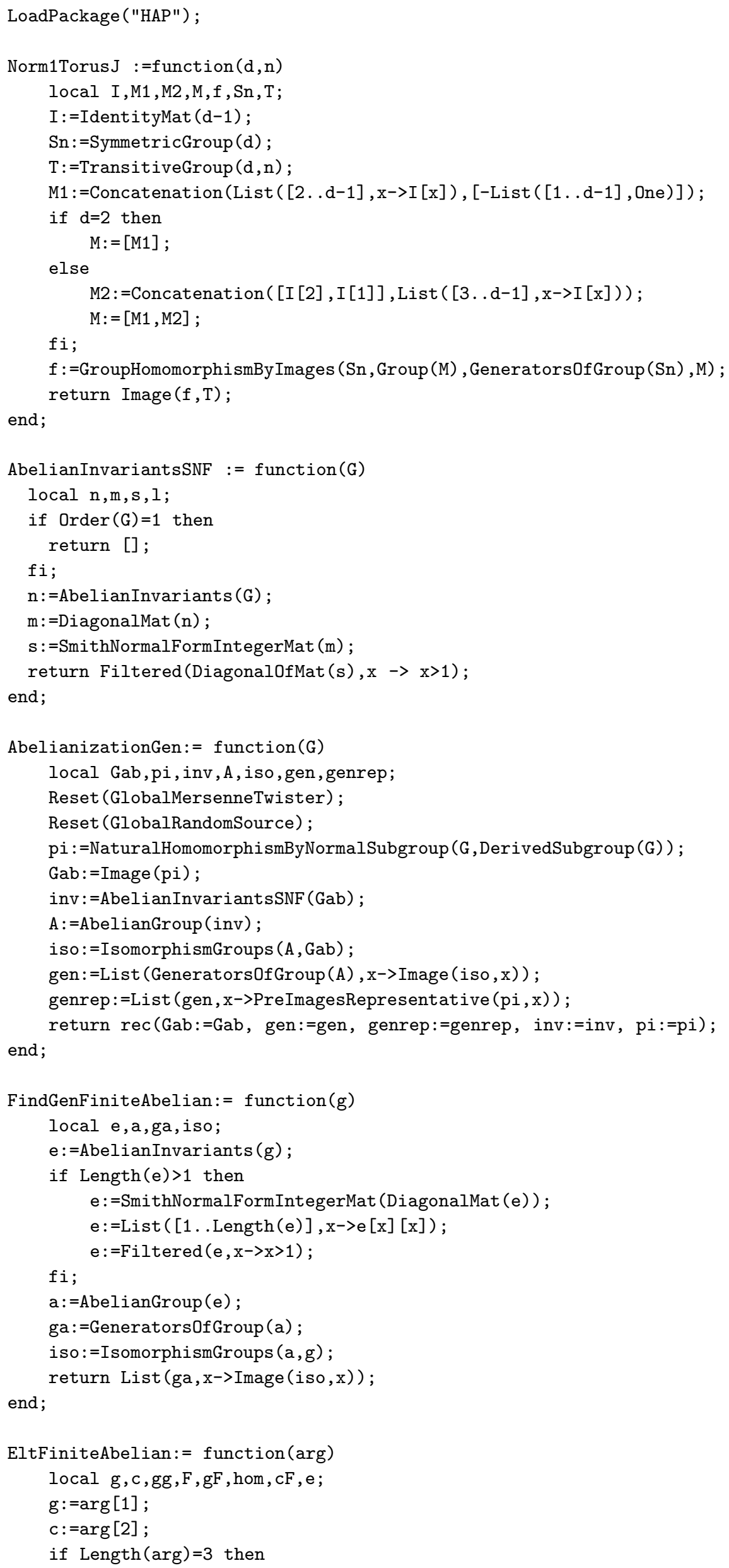




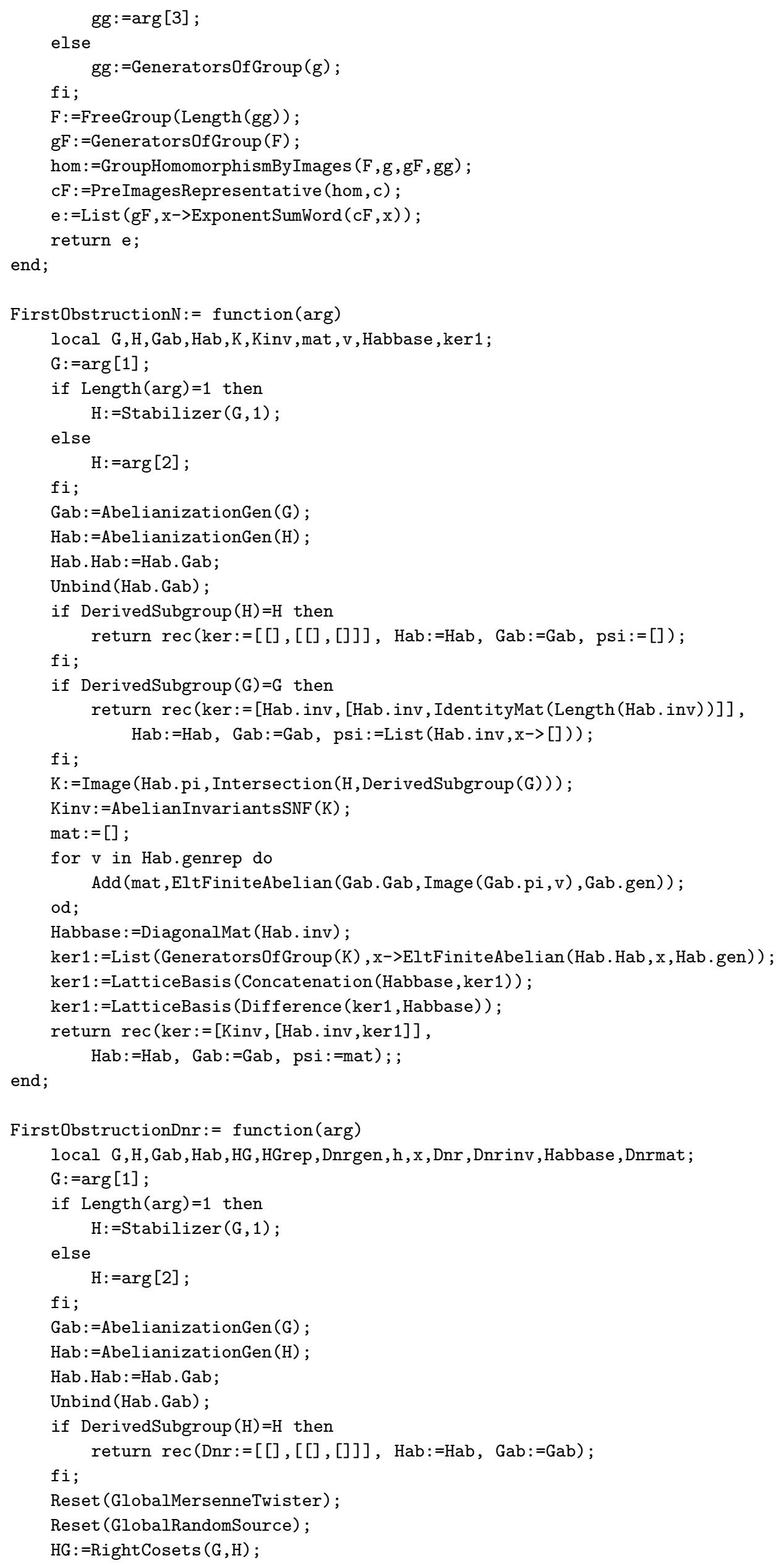




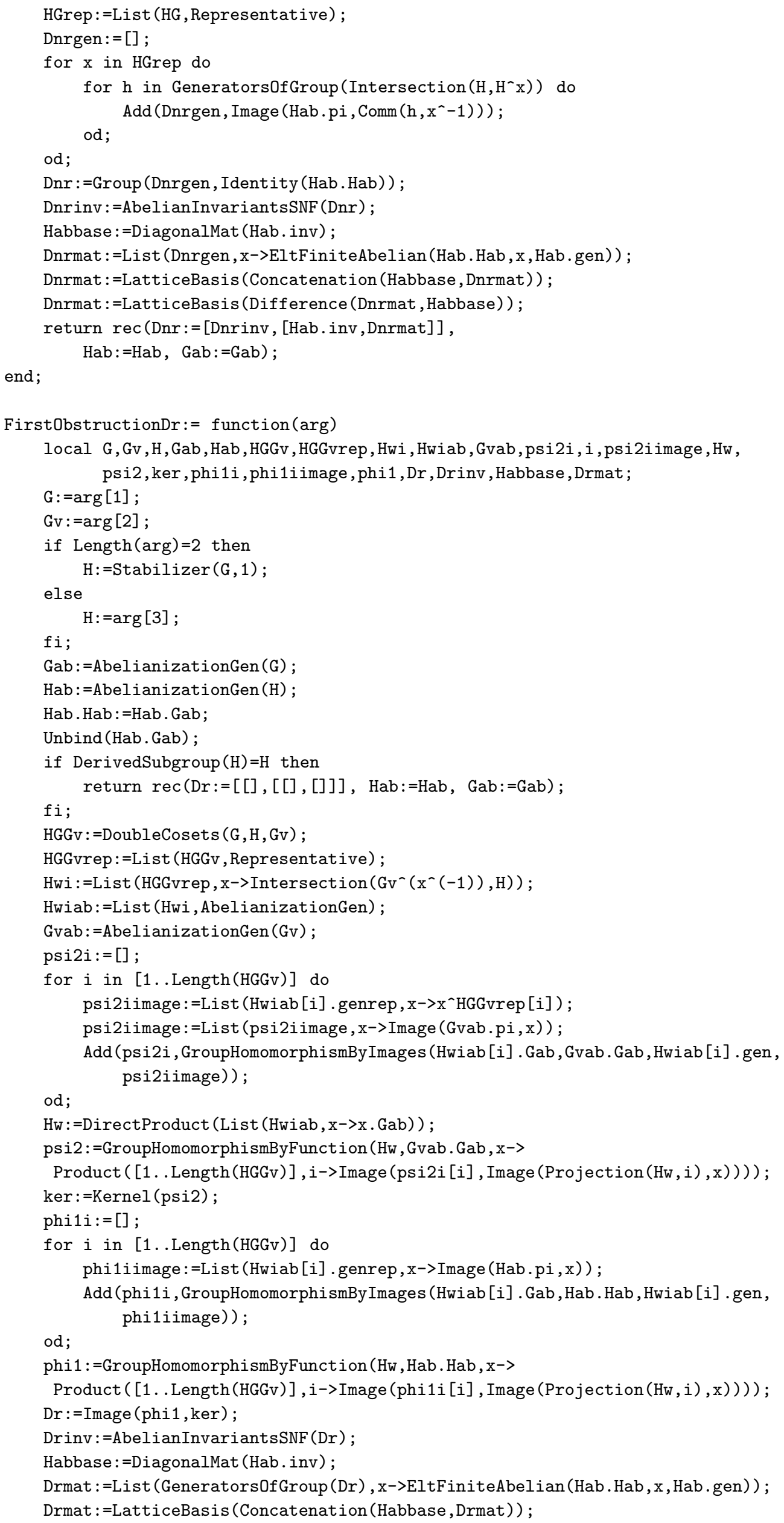


Drmat: =LatticeBasis (Difference (Drmat, Habbase)) ;

return $\operatorname{rec}(\operatorname{Dr}:=[$ Drinv, [Hab. inv, Drmat] $]$, $\mathrm{Hab}:=\mathrm{Hab}, \mathrm{Gab}:=\mathrm{Gab})$;

end;

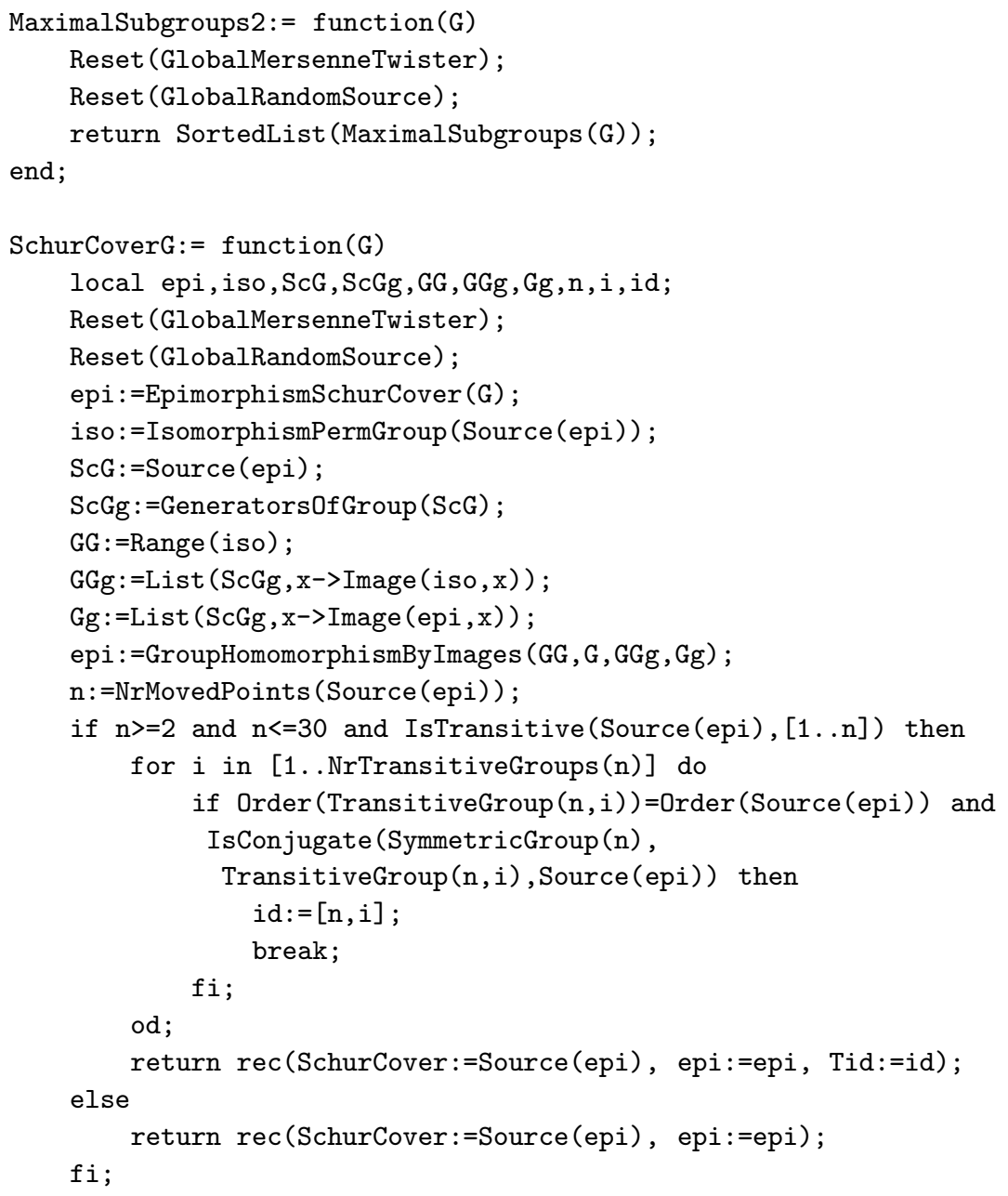




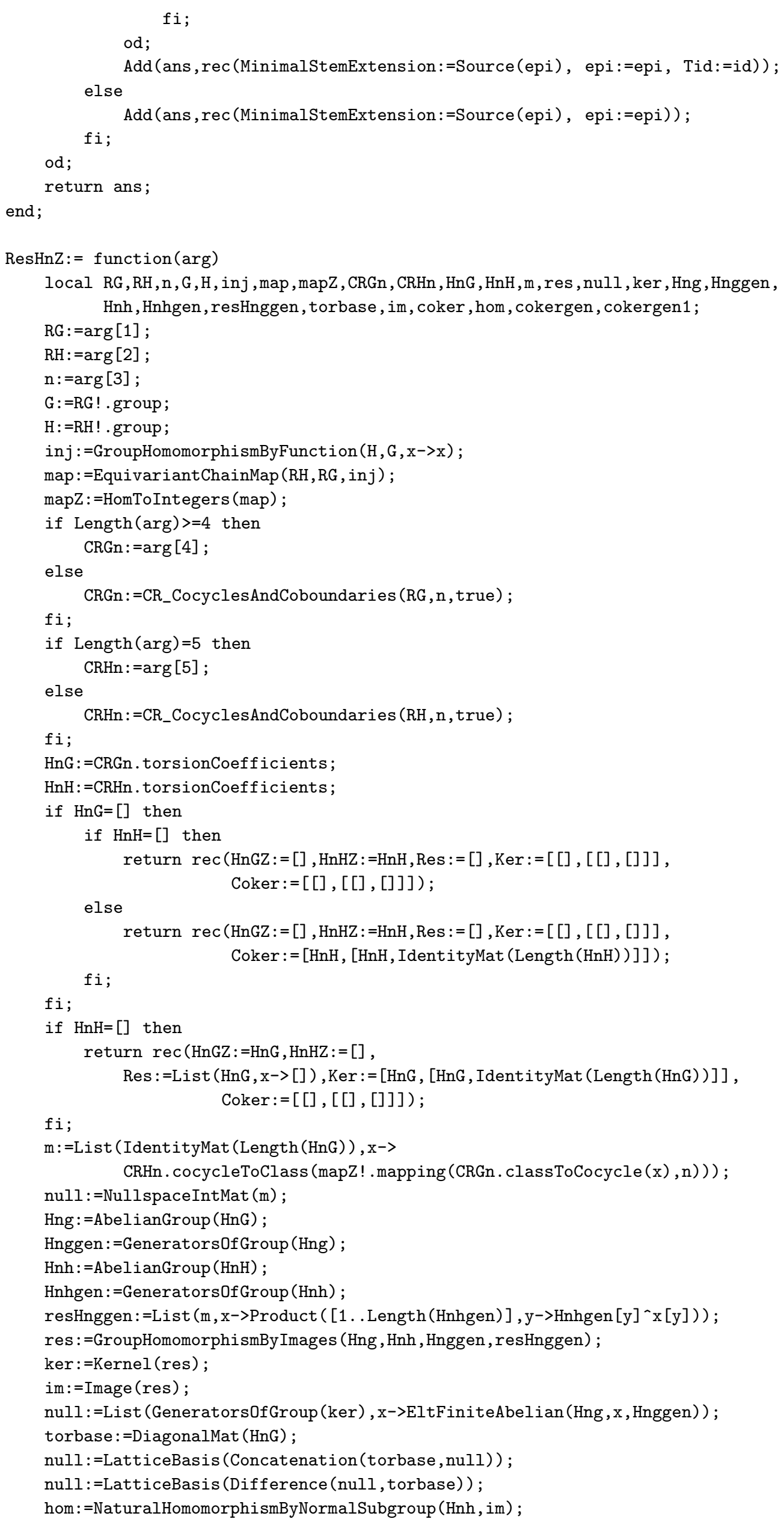


coker: =Image (hom);

if $\operatorname{Order}($ coker $)=1$ then

return $\operatorname{rec}(\mathrm{HnGZ}:=\mathrm{HnG}, \mathrm{HnHZ}:=\mathrm{HnH}$, Res $:=\mathrm{m}$,

Ker: $=[$ AbelianInvariantsSNF (ker), $[\mathrm{HnG}$, null] $]$, Coker $:=[[],[\mathrm{HnH},[]]])$;

fi;

cokergen: =FindGenFiniteAbelian (coker);

cokergen 1 : =List (cokergen, $\mathrm{x}->$ Representative (PreImages (hom , $\mathrm{x}$ ))) ;

cokergen 1 : =List (cokergen $1, \mathrm{x}->$ EltFiniteAbelian (Hnh, $\mathrm{x}$, Hnhgen)) ;

return $\operatorname{rec}(\mathrm{HnGZ}:=\mathrm{HnG}, \mathrm{HnHZ}:=\mathrm{HnH}$, Res $:=\mathrm{m}$,

Ker : $=[$ AbelianInvariantsSNF (ker), [HnG, null] $]$,

Coker:=[AbelianInvariants (coker), [HnH, cokergen1] $])$;

end;

CosetRepresentationTid:= function $(\mathrm{G}, \mathrm{H})$

local $\mathrm{Gg}, \mathrm{HG}, \mathrm{HGg}, \mathrm{HGgr}, \mathrm{n}$, i, id;

$\mathrm{Gg}:=$ GeneratorsOfGroup $(\mathrm{G})$;

$\mathrm{HG}:=\operatorname{RightCosets}(\mathrm{G}, \mathrm{H})$;

$\mathrm{HGg}$ : =List (Gg, $\mathrm{x}->$ Permutation ( $\mathrm{x}, \mathrm{HG}$, OnRight)) ;

HGgr : $=\operatorname{Group}(\mathrm{HGg},())$;

$\mathrm{n}:=\operatorname{Index}(\mathrm{G}, \mathrm{H})$;

if $\mathrm{n}=1$ then

id: $=[1,1]$;

elif $n<=30$ then

for $i$ in [1..NrTransitiveGroups(n)] do

if $\operatorname{Order}(\operatorname{TransitiveGroup}(\mathrm{n}, \mathrm{i}))=\operatorname{Order}(\mathrm{HGgr})$ and

IsConjugate (SymmetricGroup (n), TransitiveGroup (n,i), HGgr) then

id: $=[n, i]$;

break;

fi;

od;

else

id: $=f$ ail

fi;

return id;

end;

AlwaysHNPholds:= function(Tid)

local n,i, tbl, tbl4, tbl6, tbl8, tbl9, tbl10, tbl14, tbl15;

tbl4: $=[2,4]$;

$\operatorname{tbl} 6:=[4,12]$;

tbl8: $=[2,3,4,9,11,13,14,15,19,21,22,31,32,37,38]$;

tbl9: $=[2,5,7,9,11,14,23]$;

tbl10: $=[7,26,32]$;

tbl14: $=[30]$;

tbl15: $=[9,14]$;

tbl: : $[[],[],[], t b l 4,[], t b l 6,[], t b 18, t b 19, t b l 10,[],[],[], t b l 14, t b l 15]$;

if Tid=fail then

return fail;

fi;

$\mathrm{n}:=\operatorname{Tid}[1]$;

$i:=\operatorname{Tid}[2]$;

if IsPrime(n) or $\mathrm{n}=1$ then

return true;

elif $n=12$ or $n>15$ then

return fail;

elif $i$ in tbl[n] then

return false;

else

return true;

fi;

end;

IsMetacyclic: $=$ function $(G)$ 


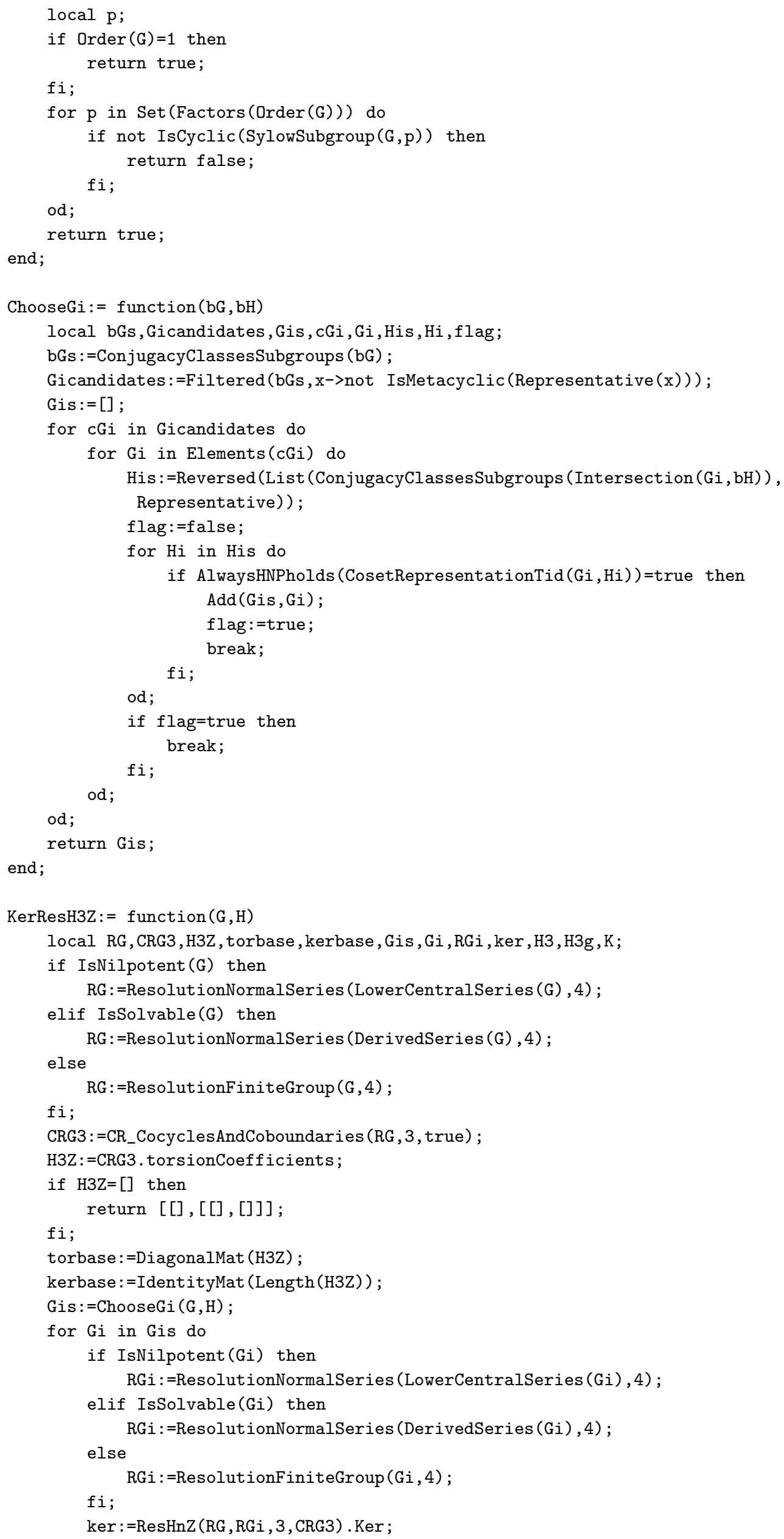


kerbase:=LatticeIntersection (kerbase,Union(ker [2] [2] , torbase)) ;

kerbase: =LatticeBasis (kerbase);

od;

kerbase:=LatticeBasis (Difference (kerbase, torbase)) ;

H3: =AbelianGroup (H3Z);

$\mathrm{H} 3 \mathrm{~g}:=$ GeneratorsOfGroup (H3);

$\mathrm{K}:=\operatorname{Group}(\operatorname{List}($ kerbase, $\mathrm{x}->\operatorname{Product}([1 \ldots \operatorname{Length}(\mathrm{x})], \mathrm{y}->\mathrm{H} 3 \mathrm{~g}[\mathrm{y}] \wedge \mathrm{x}[\mathrm{y}]$ )) , Identity (H3)) ;

return [AbelianInvariantsSNF $(\mathrm{K}),[\mathrm{H} 3 \mathrm{Z}$, kerbase $]]$;

end;

\section{REFERENCES}

[Bar81a] H.-J. Bartels, Zur Arithmetik von Konjugationsklassen in algebraischen Gruppen, J. Algebra 70 (1981) $179-199$.

[Bar81b] H.-J. Bartels, Zur Arithmetik von Diedergruppenerweiterungen, Math. Ann. 256 (1981) 465-473.

[BT82] F. R. Beyl, J. Tappe, Group extensions, representations, and the Schur multiplicator, Lecture Notes in Mathematics, 958. Springer-Verlag, Berlin-New York, 1982.

[But93] G. Butler, The transitive groups of degree fourteen and fifteen, J. Symbolic Comput. 16 (1993) 413-422.

[BM83] G. Butler, J. McKay, The transitive groups of degree up to eleven, Comm. Algebra 11 (1983) 863-911.

[CT07] J.-L. Colliot-Thélène, Lectures on Linear Algebraic Groups, Beijing lectures, Moning side centre, April 2007, https://www.math.u-psud.fr/ colliot/BeijingLectures2Juin07.pdf.

[CTHS05] J.-L. Colliot-Thélène, D. Harari, A. N. Skorobogatov, Compactification équivariante d'un tore (d'après Brylinski et Künnemann), Expo. Math. 23 (2005) 161-170.

[CTS77] J.-L. Colliot-Thélène, J.-J. Sansuc, La R-équivalence sur les tores, Ann. Sci. École Norm. Sup. (4) 10 (1977) 175-229.

[CTS87] J.-L. Colliot-Thélène, J.-J. Sansuc, Principal homogeneous spaces under flasque tori: Applications, J. Algebra 106 (1987) 148-205.

[CTS07] J.-L. Colliot-Thélène, J.-J. Sansuc, The rationality problem for fields of invariants under linear algebraic groups (with special regards to the Brauer group), Algebraic groups and homogeneous spaces, 113-186, Tata Inst. Fund. Res. Stud. Math., 19, Tata Inst. Fund. Res., Mumbai, 2007.

[CK00] A. Cortella, B. Kunyavskii, Rationality problem for generic tori in simple groups, J. Algebra 225 (2000) $771-793$.

[DM96] J. D. Dixon, B. Mortimer, Permutation groups, Graduate Texts in Mathematics, 163. Springer-Verlag, New York, 1996. xii+346 pp.

[Dra89] Yu. A. Drakokhrust, On the complete obstruction to the Hasse principle, (Russian) Dokl. Akad. Nauk BSSR 30 (1986) 5-8; translation in Amer. Math. Soc. Transl. (2) 143 (1989) 29-34.

[DP87] Yu. A. Drakokhrust, V. P. Platonov, The Hasse norm principle for algebraic number fields, (Russian) Izv. Akad. Nauk SSSR Ser. Mat. 50 (1986) 946-968; translation in Math. USSR-Izv. 29 (1987) 299-322.

[End11] S. Endo, The rationality problem for norm one tori, Nagoya Math. J. 202 (2011) 83-106.

[EK17] S. Endo, M. Kang, Function fields of algebraic tori revisited, Asian J. Math. 21 (2017) $197-224$.

[EM73] S. Endo, T. Miyata, Invariants of finite abelian groups, J. Math. Soc. Japan 25 (1973) 7-26.

[EM75] S. Endo, T. Miyata, On a classification of the function fields of algebraic tori, Nagoya Math. J. 56 (1975) 85-104. Corrigenda: Nagoya Math. J. 79 (1980) 187-190.

[Flo] M. Florence, Non rationality of some norm-one tori, preprint (2006).

[GAP] The GAP Group, GAP - Groups, Algorithms, and Programming, Version 4.9.3; 2018. (http://www.gap-system.org).

[Ger77] F. Gerth III, The Hasse norm principle in metacyclic extensions of number fields, J. London Math. Soc. (2) 16 (1977) 203-208.

[Ger78] F. Gerth III, The Hasse norm principle in cyclotomic number fields, J. Reine Angew. Math. 303/304 (1978) 249-252.

[GLS98] D. Gorenstein, R. Lyons, R. Solomon, The classification of the finite simple groups, Number 3, Part I, Chapter A: Almost simple $\mathcal{K}$-groups, Mathematical Surveys and Monographs, 40.3, American Mathematical Society, Providence, RI, 1998, xvi+419 pp.

[Gur78a] S. Gurak, On the Hasse norm principle, J. Reine Angew. Math. 299/300 (1978) 16-27.

[Gur78b] S. Gurak, The Hasse norm principle in non-abelian extensions, J. Reine Angew. Math. 303/304 (1978) $314-318$.

[Gur80] S. Gurak, The Hasse norm principle in a compositum of radical extensions, J. London Math. Soc. (2) 22 (1980) 385-397.

[HAP] G. Ellis, The GAP package HAP, version 1.12.6, available from http://www.gap-system.org/Packages/hap.html

[HHY20] S. Hasegawa, A. Hoshi, A. Yamasaki, Rationality problem for norm one tori in small dimensions, Math. Comp. 89 (2020) 923-940.

[Has31] H. Hasse, Beweis eines Satzes und Wiederlegung einer Vermutung über das allgemeine Normenrestsymbol, Nachrichten von der Gesellschaft der Wissenschaften zu Göttingen, Mathematisch-Physikalische Klasse (1931) 64-69.

[Hir64] H. Hironaka, Resolution of singularities of an algebraic variety over a field of characteristic zero. I, II., Ann. of Math. (2) 79 (1964) 109-203; 205-326.

[HY17] A. Hoshi, A. Yamasaki, Rationality problem for algebraic tori, Mem. Amer. Math. Soc. 248 (2017) no. 1176, v+215 pp.

[HY21] A. Hoshi, A. Yamasaki, Rationality problem for norm one tori, Israel J. Math. 241 (2021) 849-867.

[Hür84] W. Hürlimann, On algebraic tori of norm type, Comment. Math. Helv. 59 (1984) 539-549.

[Kan12] M. Kang, Retract rational fields, J. Algebra 349 (2012) 22-37.

[Mer08] A. Merkurjev, R-equivalence on three-dimensional tori and zero-cycles, Algebra Number Theory 2 (2008) 69-89.

[Kap87] G. Karpilovsky, The Schur multiplier, London Mathematical Society Monographs. New Series, 2. The Clarendon Press, Oxford University Press, New York, 1987.

[KMRT98] M.-A. Knus, A. Merkurjev, M. Rost, J.-P. Tignol, The book of involu-tions, Amer. Math. Soc., Providence, 1998.

[Kun84] B. E. Kunyavskii, Arithmetic properties of three-dimensional algebraic tori, (Russian) Integral lattices and finite linear groups, Zap. Nauchn. Sem. Leningrad. Otdel. Mat. Inst. Steklov. (LOMI) 116 (1982) 102-107, 163; translation in J. Soviet Math. 26 (1984) 1898-1901. 
[Kun90] B. E. Kunyavskii, Three-dimensional algebraic tori, Selecta Math. Soviet. 9 (1990) 1-21.

[Kun07] B. E. Kunyavskii, Algebraic tori — thirty years after, Vestnik Samara State Univ. (2007) 198-214.

[LL00] N. Lemire, M. Lorenz, On certain lattices associated with generic division algebras, J. Group Theory 3 (2000) 385-405.

[LPR06] N. Lemire, V. L. Popov, Z. Reichstein, Cayley groups, J. Amer. Math. Soc. 19 (2006) 921-967.

[LeB95] L. Le Bruyn, Generic norm one tori, Nieuw Arch. Wisk. (4) 13 (1995) 401-407.

[Len74] H. W. Lenstra, Jr., Rational functions invariant under a finite abelian group, Invent. Math. 25 (1974) $299-325$.

[Lor05] M. Lorenz, Multiplicative invariant theory, Encyclopaedia Math. Sci., vol. 135, Springer-Verlag, Berlin, 2005.

[Mac20] A. Macedo, The Hasse norm principle for $A_{n}$-extensions, J. Number Theory 211 (2020) 500-512.

[Mac] A. Macedo, On the obstruction to the Hasse principle for multinorm equations, arXiv:1912.11941

[MN] A. Macedo, R. Newton, Explicit methods for the Hasse norm principle and applications to $A_{n}$ and $S_{n}$ extensions, arXiv:1906.03730.

[Man74] Yu. I. Manin, Cubic forms: algebra, geometry, arithmetic, North-Holland Mathematical Library 4, North-Holland, Amsterdam, 1974.

[Maz82] P. Mazet, Sur les multiplicateurs de Schur des groupes de Mathieu, (French) J. Algebra 77 (1982) $552-576$.

[Ono61] T. Ono, Arithmetic of algebraic tori, Ann. of Math. (2) 74 (1961) 101-139.

[Ono63] T. Ono, On the Tamagawa number of algebraic tori, Ann. of Math. (2) 78 (1963) 47-73.

[Ono65] T. Ono, On the relative theory of Tamagawa numbers, Ann. of Math. (2) 82 (1965) 88-111.

[Opo80] H. Opolka, Zur Auflösung zahlentheoretischer Knoten, Math. Z. 173 (1980) 95-103.

[Pla82] V. P. Platonov, Arithmetic theory of algebraic groups, (Russian) Uspekhi Mat. Nauk 37 (1982) 3-54; translation in Russian Math. Surveys 37 (1982) 1-62.

[PD85a] V. P. Platonov, Yu. A. Drakokhrust, On the Hasse principle for algebraic number fields, (Russian) Dokl. Akad. Nauk SSSR 281 (1985) 793-797; translation in Soviet Math. Dokl. 31 (1985) 349-353.

[PD85b] V. P. Platonov, Yu. A. Drakokhrust, The Hasse norm principle for primary extensions of algebraic number fields, (Russian) Dokl. Akad. Nauk SSSR 285 (1985) 812-815; translation in Soviet Math. Dokl. 32 (1985) 789-792.

[PR94] V. P. Platonov, A. Rapinchuk, Algebraic groups and number theory, Translated from the 1991 Russian original by Rachel Rowen, Pure and applied mathematics, 139, Academic Press, 1994.

[Rob96] D. J. S. Robinson, A course in the theory of groups, Second edition. Graduate Texts in Mathematics, 80. Springer-Verlag, New York, 1996.

[Roy87] G. F. Royle, The transitive groups of degree twelve, J. Symbolic Comput. 4 (1987) 255-268.

[Sal84] D. J. Saltman, Retract rational fields and cyclic Galois extensions, Israel J. Math. 47 (1984) 165-215.

[Sal99] D. J. Saltman, Lectures on division algebras, CBMS Regional Conference Series in Mathematics, 94, Published by American Mathematical Society, Providence, RI; on behalf of Conference Board of the Mathematical Sciences, Washington, DC, 1999. viii+120 pp.

[San81] J.-J. Sansuc, Groupe de Brauer et arithmétique des groupes algébriques linéaires sur un corps de nombres, (French) J. Reine Angew. Math. 327 (1981) 12-80.

[Swa83] R. G. Swan, Noether's problem in Galois theory, Emmy Noether in Bryn Mawr (Bryn Mawr, Pa., 1982), 21-40, Springer, New York-Berlin, 1983.

[Swa10] R. G. Swan, The flabby class group of a finite cyclic group, Fourth International Congress of Chinese Mathematicians, 259-269, AMS/IP Stud. Adv. Math., 48, Amer. Math. Soc., Providence, RI, 2010.

[Tat67] J. Tate, Global class field theory, Algebraic Number Theory, Proceedings of an instructional conference organized by the London Mathematical Society (a NATO Advanced Study Institute) with the support of the International Mathematical Union, Edited by J. W. S. Cassels and A. Fröhlich, 162-203, Academic Press, London; Thompson Book Co., Inc., Washington, D.C. 1967.

[Vos67] V. E. Voskresenskii, On two-dimensional algebraic tori II, (Russian) Izv. Akad. Nauk SSSR Ser. Mat. 31 (1967) 711-716; translation in Math. USSR-Izv. 1 (1967) 691-696.

[Vos69] V. E. Voskresenskii, The birational equivalence of linear algebraic groups, (Russian) Dokl. Akad. Nauk SSSR 188 (1969) 978-981; erratum, ibid. 1911969 nos., 1, 2, 3, vii; translation in Soviet Math. Dokl. 10 (1969) 1212-1215.

[Vos70] V. E. Voskresenskii, Birational properties of linear algebraic groups, (Russian) Izv. Akad. Nauk SSSR Ser. Mat. 34 (1970) 3-19; translation in Math. USSR-Izv. 4 (1970) 1-17.

[Vos74] V. E. Voskresenskii, Stable equivalence of algebraic tori, (Russian) Izv. Akad. Nauk SSSR Ser. Mat. 38 (1974) 3-10; translation in Math. USSR-Izv. 8 (1974) 1-7.

[Vos83] V. E. Voskresenskii, Projective invariant Demazure models, (Russian) Izv. Akad. Nauk SSSR Ser. Mat. 46 (1982) 195-210, 431; translation in Math USSR-Izv. 20 (1983) 189-202.

[Vos88] V. E. Voskresenskii, Maximal tori without affect in semisimple algebraic groups, (Russian) Mat. Zametki 44 (1988) 309-318; translation in Math. Notes 44 (1988) 651-655.

[Vos98] V. E. Voskresenskii, Algebraic groups and their birational invariants, Translated from the Russian manuscript by Boris Kunyavskii, Translations of Mathematical Monographs, 179. American Mathematical Society, Providence, RI, 1998.

[VK84] V. E. Voskresenskii, B. E. Kunyavskii, Maximal tori in semisimple algebraic groups, Kuibyshev State Inst., Kuibyshev (1984). Deposited in VINITI March 5, 1984, No. 1269-84 Dep. (Ref. Zh. Mat. (1984), 7A405 Dep.).

[Yam12] A. Yamasaki, Negative solutions to three-dimensional monomial Noether problem, J. Algebra 370 (2012) 46-78.

Department of Mathematics, Niigata University, Niigata 950-2181, Japan

Email address: hoshi@math.sc.niigata-u.ac.jp

Graduate School of Science and Technology, Nitgata University, Niigata 950-2181, Japan

Email address: kanai@m.sc.niigata-u.ac.jp

Department of Mathematics, Kyoto University, Kyoto 606-8502, Japan

Email address: aiichi.yamasaki@gmail.com 